\title{
On the Formation and Evolution of Dwarf Galaxies in Tidal Tails
}

\author{
Dissertation \\ zur Erlangung des Doktorgrades \\ der Mathematisch-Naturwissenschaftlichen Fakultäten \\ der Georg-August Universität zu Göttingen
}

\author{
vorgelegt von \\ Peter Michael Weilbacher \\ aus Holzminden
}

Göttingen 2002 
Referent: Priv. Doz. Dr. U. Fritze-v. Alvensleben Korreferent: Prof. Dr. K.J. Fricke

Tag der mündlichen Prüfung: 24. Oktober 2002 


\begin{abstract}
Tidal Dwarf Galaxies (TDGs) are a class of dwarf galaxies that form in tidal tails of interacting giant galaxies during close encounters or merging. As such they offer the possibility of observing galaxy formation in the local universe. Previously, only a few objects of this kind were known observationally.

The objective of this thesis is to combine, for the first time, photometric and spectroscopic observations with evolutionary synthesis models in the wavelength range from the optical to the near-infrared to analyze the stellar populations seen in the first reasonably large sample of Tidal Dwarfs compiled in the course of this work.

For this purpose, we selected 14 interacting and merging galaxies. From optical imaging and comparison with models specifically adapted to the star formation history of TDGs, a total of 44 knots in the tidal features were selected as good candidates for TDGs. Subsequently, we employed optical spectroscopy to confirm the association of the knots with the main galaxies and to derive important properties of the objects, like oxygen abundance, Balmer line luminosity and equivalent width, needed as input for further modeling. Additionally, we could show with the spectroscopic data that 13 TDG candidates have velocity fields which suggest that they are dynamically decoupled from their surrounding tidal tail and therefore true Tidal Dwarf Galaxies. Finally, we combined new near-infrared imaging with the optical data for the comparison with new, refined evolutionary synthesis models and showed that the TDG candidates have stellar masses of dwarf galaxies and star formation rates showing them to be experiencing considerable starbursts. While they have strong contributions of young stars to their stellar mass we conclude that most of them have been formed as stellar clumps rather than by the collapse of giant gas clouds.
\end{abstract}





\section{Contents}

0 Introduction 9

1 Galaxy Formation . . . . . . . . . . . . . . . . 11

2 Tidal Dwarf Galaxies . . . . . . . . . . . . . . . . 13

2.1 A Brief History of TDG Research . . . . . . . . . . . . 13

2.2 Formation scenario for TDGs . . . . . . . . . 16

2.3 TDGs as genuine galaxies . . . . . . . . . . 16

2.4 TDGs as young galaxies? . . . . . . . . . . . . . 18

2.5 Some notes on the sample selection . . . . . . . . . 19

3 Thesis Outline . . . . . . . . . . . . . . . . . . 20

I Sample Selection: Optical Photometry and Models 25

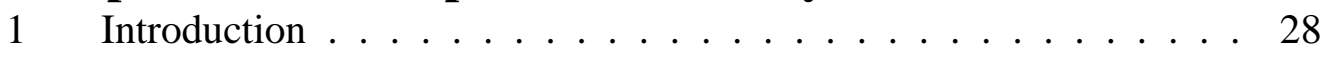

2 Observations and data reduction $\ldots \ldots \ldots \ldots$

$2.1 \quad$ Data and Calibration $\ldots \ldots \ldots \ldots$

2.2 Aperture photometry . . . . . . . . . . . . . 31

3 Model . . . . . . . . . . . . . . . . . . . 32

$3.1 \quad$ Modelling the old population . . . . . . . . . . . 32

3.2 Input physics . . . . . . . . . . . . . . . . 32

3.3 TDG models . . . . . . . . . . . . . . . . . . 33

$3.4 \quad$ Model application and limitations $\ldots \ldots \ldots$

$4 \quad$ Results . . . . . . . . . . . . . . . . . . . . 37

$4.1 \quad$ AM $0529-565 \ldots \ldots \ldots \ldots \ldots$

$4.2 \quad$ AM $0537-292 \ldots \ldots \ldots$. . . . . . . . . . . . . . . 39

$4.3 \quad$ AM $0547-244 \ldots \ldots \ldots$. . . . . . . . . . . . . . 40

$4.4 \quad$ AM $0607-444 \ldots \ldots \ldots \ldots \ldots \ldots$. . . . . . . . . 41

$4.5 \quad$ AM $0642-645 \ldots \ldots \ldots \ldots \ldots \ldots$. . . . . . . . . . . . 41

4.6 AM 0748-665 . . . . . . . . . . . . . . . . . . 42

4.7 AM $1054-325 \ldots \ldots \ldots \ldots$. . . . . . . . . . . 43

$4.8 \quad$ AM $1208-273 \ldots \ldots \ldots \ldots$. . . . . . . . . . . . 44

4.9 AM $1325-292 \ldots \ldots \ldots \ldots \ldots$ 
4.10 AM $1353-272 \ldots \ldots \ldots \ldots \ldots \ldots$

5 Discussion . . . . . . . . . . . . . . 47

5.1 Background contamination . . . . . . . . . 47

5.2 Properties of the TDG candidates . . . . . . . . . . 47

5.3 Nature of the TDG candidates . . . . . . . . . . 52

6 Conclusions . . . . . . . . . . . . . . . . 54

II Results from Optical Spectroscopy $\mathbf{5 9}$

1 Introduction . . . . . . . . . . . . . . . 62

2 Observations and data analysis . . . . . . . . . 63

2.1 Spectroscopic observations . . . . . . . . . . . 63

2.2 Velocity measurements . . . . . . . . . . . . 66

2.3 Oxygen abundance measurements . . . . . . . . . 67

3 Results ....................... 68

$3.1 \quad$ Metallicity ............... 70

3.2 Internal extinction $\ldots \ldots \ldots \ldots 71$

3.3 Ho luminosities . . . . . . . . . . . . . . . . . 71

3.4 Kinematics . . . . . . . . . . . . . . 72

4 Discussion ......................... 75

4.1 TDG candidates: between giant HII regions and individual dwarfs galaxies? . . . . . . . . . . . . . 75

4.2 Velocity gradients . . . . . . . . . . . . 76

4.3 Selecting Tidal Dwarf Galaxies . . . . . . . . . . 79

5 Conclusions .................... 80

A Photometric results of the 4 new systems . . . . . . . . . 85

A.1 AM $0547-474 \ldots \ldots \ldots \ldots$. . . . . . . . 86

A.2 AM $1159-530 \ldots \ldots \ldots \ldots$. . . . . . . 87

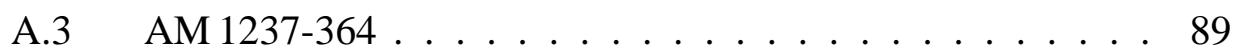

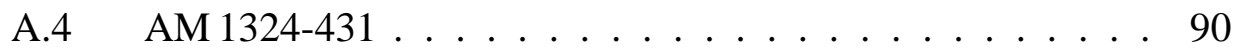

B Notes about individual systems . . . . . . . . . . . . . . 90

B.1 AM $0529-565 \ldots \ldots \ldots \ldots$

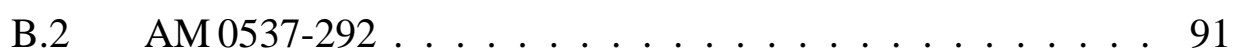

B.3 AM $0547-244 \ldots \ldots \ldots \ldots \ldots$

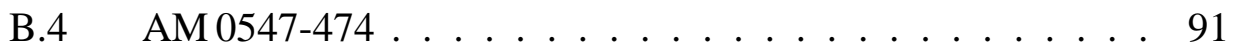

B.5 AM $0607-444 \ldots \ldots \ldots \ldots . \ldots \ldots 2$

B.6 AM $0642-645 \ldots \ldots \ldots \ldots \ldots 2 \ldots \ldots \ldots$

B.7 AM $0748-665 \ldots \ldots \ldots \ldots \ldots$

B.8 AM $1054-325 \ldots \ldots \ldots \ldots$

B.9 AM $1159-530 \ldots \ldots \ldots \ldots \ldots$

B.10 AM 1208-273 .................. 94 
B.11 AM 1237-364 . . . . . . . . . . . . . 94

B.12 AM 1324-431 . . . . . . . . . . . . . . 94

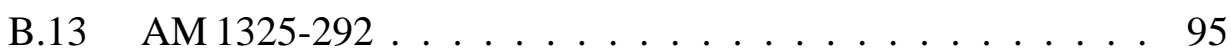

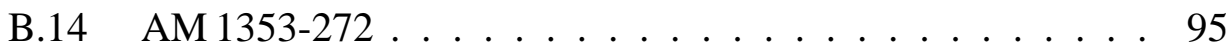

\section{Burst Properties: Optical-NIR Photometry and Models 99}

1 Introduction . . . . . . . . . . . . . . . . 102

2 Observations and data analysis . . . . . . . . . . . 103

2.1 Data reduction . . . . . . . . . . . . . . 104

2.2 Photometry . . . . . . . . . . . . . . 104

3 Model description . . . . . . . . . . . . . . . . 106

4 Comparison observations-models . . . . . . . . . . . . . . 107

$5 \quad$ Results and discussion . . . . . . . . . . . . . . . 109

5.1 Photometric properties . . . . . . . . . . . . 109

5.2 Properties derived from the models . . . . . . . . . . 111

5.3 TDG candidates with observed velocity gradients . . . . . 114

5.4 TDGs: Built from gaseous or stellar clumps? . . . . . . . 115

6 Conclusions . . . . . . . . . . . . . . . . 116

IV On Star Formation Rates in Dwarf Galaxies 119

1 Introduction . . . . . . . . . . . . . . . 121

2 Model description . . . . . . . . . . . . . . . . . . . 122

3 Effects of Metallicity . . . . . . . . . . . . . . . 123

4 Short starbursts . . . . . . . . . . . . . . . . . . . . . . . 124

5 Effects of the IMF . . . . . . . . . . . . . . . . . . . . . . . . . . . . . . .

6 Conclusions . . . . . . . . . . . . . . . . . . . . . 129

V A Spectacular Example: AM 1353-272 131

1 Introduction . . . . . . . . . . . . . . . . . . 133

2 Observations and data reduction . . . . . . . . . . . . . 134

3 Results . . . . . . . . . . . . . . . . 135

4 Discussion and Conclusions . . . . . . . . . . . . 137

\section{Summary 141}

1 Summary of the results . . . . . . . . . . . . . . . . . 143

2 Conclusions from this sample of TDGs . . . . . . . . . . . . . 145

3 Future Research . . . . . . . . . . . . . . . . . . . . 146 
Frequently used acronymns and abbreviations 151

Danksagung 153

Curriculum Vitae 155 


\section{Part 0}

\section{Introduction}





\section{Galaxy Formation}

The question how galaxies form has been a key issue in extragalactic astronomy for the last several decades. As questions of galaxy formation are best answered by observing young galaxies, it seems logical to search for these at times when the universe was still young, i.e. at high redshifts. Since Hubble's discovery that some "nebulae" are in fact galaxies like our own (Hubble 1936), the size and lightcollecting power of the telescopes has increased by several orders of magnitude, and fainter and fainter galaxies, located at larger and larger distances, closer to the epoch of galaxy formation, were discovered. By now, several galaxies with redshifts $z>5$ are known (e.g. Hu et al. 1999, Ellis et al. 2001). The current record holder in terms of spectroscopic redshift is HCM 6A with $z=6.56$, a galaxy behind the galaxy cluster Abell 370 (Hu et al. 2002). At its distance, the universe has an age of $\sim 890$ Myrs, only about $6 \%$ of its present age ${ }^{1}$. At such distances, the major obstacle is the faintness and apparent size of the galaxies. Even with the high spatial resolution of the Hubble Space Telescope (HST) distant galaxies, like those in the "Hubble Deep Fields" (Williams et al. 1996, Williams et al. 2000), cover only a few pixels on the detectors. Larger telescopes on the ground are especially hampered by the atmospheric seeing, so that distant objects appear as faint, nearly point-like sources. The above examples of high redshift galaxies could only be detected, because their luminosity is enhanced by up to a factor of 5 by gravitational lensing of the galaxy clusters behind which they are located. And still, several hours of exposure time on the world's largest optical telescope, the $10 \mathrm{~m}$-Keck telescope on Hawaii, were necessary for the spectroscopic confirmation of the redshift. This clearly shows the difficulties in studying galaxy formation in the young universe.

There are several alternative approaches, to learn about the formation of galaxies. When looking at the present-day galaxies, we see the end products of the galaxy formation process and — to some extent — "fossil records" of events in the course of their formation. It seems clear, that spiral and elliptical galaxies are formed by different processes. The dynamics and light profiles of ellipticals point to a fast process ("violent relaxation"), while spiral galaxies with their flat, more irregular, rotating disks are very likely formed by slow accretion. But it is not immediately clear, when exactly during their history these events occurred, e.g. if there was one singular epoch of galaxy formation or if galaxy formation is a continuous process extending into present times. One can then use modeling tools like e.g. evolutionary synthesis models (Tinsley 1972, Fritze-von Alvensleben et al. 1989) - connecting stellar evolution and a star formation history with observables like luminosities, colors, and gas content —, semi-analytical (White \& Rees 1978), or combined dynam-

${ }^{1}$ Computed using the "standard" parameters for a $\Lambda$-cosmology, $H_{0}=65 \mathrm{~km} \mathrm{~s}^{-1} \mathrm{Mpc}^{-1}, \Omega_{M}=$ 0.3 , and $\Omega_{\Lambda}=0.7$. 
ical and photometric (Contardo et al. 1998) models of hierarchical galaxy assembly to disentangle formation and evolution. When comparing such models with observations of local galaxies and of galaxies with a wide range in redshifts, the effects of galaxy evolution over the Hubble time can be explored and conclusions about the time and duration of the process of galaxy formation can be reached.

Another approach to look for clues to galaxy formation in present day galaxies is possible only for very nearby galaxies, in particular the dwarf galaxies in the local group. In these objects, single stars can be resolved and a color-magnitude diagram (CMD) can be produced. Using stellar evolutionary tracks and Monte Carlo simulations, synthetic CMDs can be computed (Tosi et al. 1991). The comparison of these synthetic CMDs with the observed ones (Tolstoy \& Saha 1996) can give very detailed information about the star formation history of a galaxy, to larger lookback times than using methods which only use the integrated light of galaxies for comparison. With this method Tolstoy et al. (1998) claim to have found that the local group dwarf irregular Leo A has a predominantly young stellar population, i.e. most of the stars in this galaxy seem to have formed during the last 2 Gyrs.

Yet another alternative is to search for truly young galaxies in the local universe. When Searle \& Sargent (1972) found a new class of dwarf galaxies with very blue colors of young stars and low metallicity, the blue compact dwarf galaxies (BCDs), they were considered to be candidates for young galaxies in the process of formation. With deeper observations it became clear that most of these objects were actually starbursts embedded in a low surface brightness envelope of red stars (Loose \& Thuan 1986, Kunth et al. 1988), most likely several billion years old. Very few of these objects, most notably IZw 18 (Papaderos et al. 2002) and SBS 0335-052 (Vanzi et al. 2000), are still intensely debated, because their Helium content does not differ much from the value presumed to be primordial and the metal abundance is very low (Izotov \& Thuan 1999). Legrand (2000) showed that models with a continuous very low star formation rate over a Hubble time and a recent starburst can be devised and fit some observations, including the low metal abundance. But until now no clear observational proof for stars older than 50 Myrs has been found in these two starburst galaxies and their light seems to be dominated by gaseous emission of very low metallicity. Although quite close to the Galaxy, observations do not yet show why they currently experience a starburst, or why their formation should happen only now, a Hubble time after the main epoch of galaxy formation.

Another manifestation of galaxy formation observable in the local universe are dwarf galaxies which form in tidal tails during interactions of giant galaxies up to the present day. They are now generally called Tidal Dwarf Galaxies (TDGs). 


\section{Tidal Dwarf Galaxies}

\subsection{A Brief History of TDG Research}

Without strong observational evidence, the idea that low mass galaxies could be formed during giant galaxy collisions was already put forward by Zwicky (1956). With the appearance of the first catalogs of interacting and merging galaxies (Vorontsov-Velyaminov 1959, Arp 1966), it became apparent that galaxies are not just static, isolated entities. Instead, the possibility of close encounters which radically change their morphology is quite high. Without clearly understanding how these transformations proceed dynamically, Arp (1969) found companion galaxies at the ends of spiral arms of several objects from his interacting galaxy catalog, which he thought of as "ejected" from the main galaxy, perhaps by a process similar to the ejection of jets from nuclei of active galaxies. It later turned out that these companion galaxies were in fact fairly massive companions involved in - but not produced by - the interaction. Stockton (1972) found bright companion galaxies with emission lines and young stars typical for $\mathrm{H}$ II regions close to elliptical galaxies. Stockton's hypothesis was that these are formed from gas clouds in the vicinity of the giant galaxies. Only the first paper using numerical simulations of galaxy interactions by Toomre \& Toomre (1972) allowed to finally understand how tidal tails and other features frequently observed in mergers are formed by gravitational forces.

The first thorough analysis of an apparent dwarf galaxy built in a tidal tail was presented by Schweizer (1978). He notes the young age of the stars in an area at the tip of the southern tail of the "Antennae" galaxies (NGC 4038/39), the metallicity being higher than expected for a location at such large radii, and questions, whether a dwarf galaxy built at this location might remain stable.

Only 13 years later the investigation of this specific mode of galaxy formation was taken up by Mirabel et al. (1991, 1992), who argue to have found at least one dwarf galaxy in the long tails of the "Superantennae" merger (AM 1925-724), and confirm Schweizer's observations of the dwarf galaxy in the Antennae, although the object they discuss is offset from the original knot by a few arcminutes. Brouillet et al. (1992) detect a massive molecular cloud, not associated with any stellar counterpart, in the M81 group by CO observations. Hernquist (1992) discusses all these observations and notes the possibility that more (dwarf) galaxies could be formed during the collisions of larger galaxies. At the same time he raises doubts at the tidal origin of most of the local group dwarf galaxies because of the relatively undisturbed structure of the Milky Way.

The first numerical simulations of the dynamics of interacting galaxies with regard to TDGs were performed by Barnes \& Hernquist (1992, from now on BH92) and Elmegreen et al. (1993, from now on EKT93). Their modeling techniques are dif- 
ferent and they arrive at very different conclusions. BH92 used a 3D N-body code combined with smoothed particle hydrodynamics (SPH) and followed a total of 90112 particles of which 16384 represent the gas phase. In their model, several condensations in the tails which are found to become bound objects by the end of the simulation, start from stellar knots, which later drag gas into their potential. BH92 also note that TDGs are not likely to contain a large amount of dark matter (DM), because their material is drawn from the spiral disk while the DM is thought to surround the galaxy in an extended halo. The model of EKT93, on the other hand, uses a 2D representation with a total of $\sim 94000$ particles of which $\sim 31000$ are modeled as sticky gas particles; the interacting companion is only a point mass. The one TDG in their model is a massive cloud of gas which forms at the end of the tidal tail; due to its mass, this cloud could also attract stars from the tail and so inherit some of the old stellar population from the parent disk.

These theoretical predictions motivated observers to start several new campaigns, trying to find out which of these models is (more often) realized in nature. Yoshida et al. (1994) believe to have found a TDG as a small knot with $\mathrm{H}$ II region properties $55^{\prime \prime}$ east of the merger NGC 2782 . Within this knot they measure a velocity gradient, from which they derive a rotational motion of $120 \mathrm{~km} \mathrm{~s}^{-1} \mathrm{kpc}^{-1}$ and a mass estimate of $4 \cdot 10^{8} \mathrm{M}_{\odot}$, similar to the $\mathrm{H}$ I mass of this knot. They also note, however, that the object is likely to have low metallicity, which is not typical for TDGs and would instead point to a dwarf galaxy existing before the collision. Duc \& Mirabel (1994) and Duc et al. (1997) find two TDGs in the system Arp 105, both of which are close to the upper luminosity limit of dwarf galaxies and contain most of the atomic gas in the system. Both TDGs are actively star-forming, but also contain a non-negligible amount of mass in old stars. But there also seem to be examples for both of the extreme formation scenarios from the two dynamical simulations: in NGC 5291, Duc \& Mirabel (1998) believe to have found "purely young" TDGs, that have no old stars but are built from the gas expelled by the central merger. In Arp 245, on the other hand, the TDG candidate in the north of the system seems to contain mostly old stars, and only up to $2 \%$ of its stellar population consists of young stars born in the current star formation episode (Duc et al. 2000).

Other serious attempts to investigate the TDG phenomenon in interacting galaxies were done by Hibbard et al. (1994). The authors combine H I observations with optical imaging and find two TDG candidates near the ends of the tidal tails of the giant merger NGC 7252, which seem to have properties very similar to the ones in Arp 105: they are very gas rich and also have the bluest optical colors of the whole system. Hibbard \& Mihos (1995) successfully create a dynamical N-body model of NGC 7252, and predict which parts of the tails will fall back onto the merger remnant on which timescales. Their result is that most of the tidal material remains bound to the central merger, but a significant amount of matter will not fall back 
within a Hubble time. It is expected that the two TDGs - if they remain stable entities - will attain long-lived orbits around the merger.

Some other investigations try to find TDGs in the specific environment of compact galaxy groups. Hunsberger et al. (1996) use $R$-band images of 42 groups and find 47 candidates for TDGs. Here, the main problem is that neither color nor spectroscopic information is available, and background galaxies can only be excluded statistically but not individually. Iglesias-Páramo \& Vílchez (2001) observe 16 compact groups with narrow-band $\mathrm{H} \alpha$ filters. Here, the association of the luminous $\mathrm{H}$ II regions they find and designate as TDG candidates is much more likely, due to the preselection by narrow-band images. Temporin (2001) discovers several candidates for TDGs in the tidal tails of the ultra-compact group CG J1720-67.8, and finds them to be of young age using evolutionary synthesis models for comparison. In contrast, Nishiura et al. (2002) analyze deep images of Seyfert's Sextet taken in 10 filterbands and find with the help of photometric evolution models that the tidal debris in this compact group, which is partly observed as optical fuzz in the northeast of the system, does not consist of young stars, but instead has a stellar population with an age of about 10 Gyrs. A dynamical analysis of several knots visible in the Stephan's Quintet has been carried out by Mendes de Oliveira et al. (2001) using Fabry-Perot interferometric observations. With the high spectral resolution of this technique, it could be shown that several condensations the size of dwarf galaxies seem to rotate. Two of these objects might have enough distance to the nearest giant galaxy to withstand disruption and survive.

Normal dwarf galaxies and dwarf spheroidal (dSph) galaxies in particular are observed to have large mass-to-light ratios and are therefore conventionally thought to have a high dark matter content. This should preclude the possibility that TDGs make a large contribution to the dwarf galaxy population. Kroupa (1997, 1998), however, suggests that dSphs are formed as TDGs and have only little DM content. Instead, their large apparent mass-to-light ratio derived from their velocity dispersion could be due to specific dynamics, where dSphs are not in dynamical equilibrium, non-spherical, and have non-isotropic velocity dispersions. While the Milky Way is not generally called a merger remnant, other studies (e.g. Lynden-Bell \& Lynden-Bell 1995) have argued for the existence of tidally created dwarf galaxies among the members of the local group, because some of them are connected to nearby faint tidal features like the Magellanic stream. An analytical model by Okazaki \& Taniguchi (2000) describes the formation of TDGs depending on their environment. They find that if a few dwarf galaxies would form in every galaxy collision, this would explain the morphology-density relation of dwarf galaxies observed in galaxy clusters.

All spectroscopic observations of TDGs showed that the luminosity-metallicity correlation found for normal dwarf galaxies does not hold up for TDGs. While dwarf galaxies have lower oxygen abundances for lower luminosities, TDGs have an ap- 
proximately constant metallicity of around $1 / 3$ of the solar value as determined from gaseous emission lines (see e.g. Duc et al. 2000, or Fig. 31 on page 70 in Paper II). On this basis, Hunter et al. (2000) confirm the metallicity of TDGs as one of the best criteria to find old dwarf galaxies which might have formed during galaxy collisions a few Gyrs ago. Supplementing this criterion with estimates about rotational properties and stellar populations, they also present a list of nearby dwarf irregulars which might be good candidates for old TDGs.

As a final remark on the history of TDGs it may be noted that this is the third doctoral thesis dealing with the specific topic of Tidal Dwarf Galaxies. While the work by Duc (1995) concentrated on the detailed, multi-wavelength analysis of the first examples of TDGs discovered in prototypical interacting systems, Hunsberger (1998) examined the dwarf galaxy population in 42 Hickson compact groups by means of $R$-band imaging and estimated the effect of TDGs on the luminosity function in these groups. This thesis for the first time provides a sample of TDG candidates which are observed in both the optical and the near-infrared with imaging and spectroscopy and analyses them with photometric and spectral evolutionary synthesis models.

\subsection{Formation scenario for TDGs}

From the observational evidence and especially the theoretical modeling of TDGs presented in the previous section, a first rough formation sequence of Tidal Dwarfs can be drafted:

An interaction occurs between two or more galaxies, of which at least one has to be a disk galaxy so that the interaction is able to produce tidal tails. One or more stellar or gaseous condensations appear within the tails, born from gravitational instabilities. If massive enough, the gravitational potential of these condensations will attract the surrounding matter. When a condensation has condensed enough, the neutral gas will be transformed into molecular gas and star formation can start on top of a possible "old" stellar population. If this proto dwarf galaxy withstands the gravitational forces of the surrounding matter and the supernova explosions in the course of its star formation episode, and does not fall back into the parent galaxy, it will survive as an independent "normal" dwarf galaxy as soon as the remaining matter in the tidal features falls back onto the remnant or thins out and disappears.

\subsection{TDGs as genuine galaxies}

Are Tidal Dwarfs real galaxies? Do they survive long enough to deserve being called "galaxies"? For normal galaxies these questions are easily answered. Most galaxies are very isolated, without much matter nearby to disturb their stability. 
Some starburst galaxies may lose much of their gas by strong stellar wind and supernova driven outflows, but in most cases there is no doubt that the stellar component will remain bound and stable, even without gas, for several billion years. The same is true for normal dwarf galaxies in less dense environments. And while dwarf galaxies have dark matter halos, they are already easily disrupted by passages of other galaxies (see e.g. Papaderos et al. 2001).

For TDGs as observed today, these questions are more difficult to answer. They are embedded in a tidal tail and, most probably, do not have massive DM halos. Tidal forces of the parent galaxy disturb their gravitational field, strong star formation might blow away the recently accreted gas, and some of the TDGs may even fall back into the central merger (Hibbard \& Mihos 1995). A definition, first proposed by Duc et al. (2000), and clarified by Weilbacher \& Duc (2001), tries to make sure that only those objects are called Tidal Dwarf Galaxies, which deserve the classification as "galaxy":

A Tidal Dwarf Galaxy is a self-gravitating entity of dwarf-galaxy mass built from the tidal material expelled during interactions.

When this definition was issued, only very few objects were known for which the term "genuine TDG" would have been appropriate, namely those where internal kinematics had already been observed, i.e. the knots NGC 5291 a and i, Arp $105 \mathrm{~N}$ and S, and perhaps also Arp $245 \mathrm{~N}$. With the exception of Arp $105 \mathrm{~N}$, molecular gas (CO) was also detected in all of these objects by Braine et al. (2000, 2001). The CO spectra show that the molecular gas has the same kinematics (velocity and line width) as the neutral $\mathrm{HI}$ gas at the same position. One explanation is that the molecular gas is forming in situ from the neutral gas, which means that the condensation is bound and massive enough to transform the neutral gas into molecular gas. An alternative explanation would be that the molecular gas clouds originate from the same region in the parent galaxy as the neutral gas and have remained stable on the way from the parent disk to the tidal tail. In both cases the $\mathrm{CO}$ is likely to have the same kinematics as the $\mathrm{HI}$.

On the other hand, it was shown by Hibbard et al. (2001) that the "prototype" of all TDGs, the object in the southern tail of the Antennae, may not be a real TDG. At the positions given by Schweizer (1978) and Mirabel et al. (1992), they find two peaks in the gas density, but the very massive apparent condensation is caused by a projection of a sharp bend near the end of the tail. And although Braine et al. may have detected $\mathrm{CO}$ at the position given by Mirabel et al., it cannot be decided from 
present observations if the two smaller knots are kinematically decoupled from the tail.

\subsection{TDGs as young galaxies?}

As opposed to the controversial cases of young BCDs and although the detailed formation processes are not known, it is clear that the formation date of TDGs is defined by the interaction of their parents. One knows they are forming now and can observe in detail the processes which lead to the condensation and the starburst in the tails.

But are these objects truly young? Most of the examples of TDGs discussed in Section 2.1 seem to contain old stars. Following the definition of Huchra (2001), a galaxy is young, if it has converted more than $50 \%$ of its stellar mass into stars during the last 100 Myrs. In this sense, objects like Arp $245 \mathrm{~N}$ are not young galaxies, but some of the TDGs in NGC 5291, where the content of old stars seems to be low, may be truly young. But even there, Fritze-von Alvensleben et al. (1998) showed with evolutionary synthesis models that an old population of as much as $10 \times$ the mass of the current starburst could be hidden in these "purely young" objects depending on the timescale of the starburst.

One could also say that a TDG forming in the scenario of EKT93 is young while the knots in the scenario of BH92 are just reconfigurations of old stars from the parent disk and hence, cannot be called young regarding the stellar population. This also depends on the time during their evolution at which we observe the condensations, as BH92's knots can also attract surrounding gas. And as observations of e.g. NGC $7252 \mathrm{E}$ show, knots of old stars can also have a massive gas cloud associated with them. This issue is not really clear in general but has to be decided on a case by case basis. Pure stellar knots are young, however, in the sense that they formed recently as structural entities, because they are detected within tidal structures or debris around merging galaxies. Other than BCDs, which could be called chemically young because their metal abundance does not indicate much enrichment, another definition of "young" attributable to TDGs could therefore be dynamically young.

It should be made clear that the conclusions reached about the formation of TDGs in the local universe cannot automatically be applied to an epoch of galaxy formation in the early universe, where both the intergalactic radiation field and the metal abundances of the star-forming gas were very different. Certain processes, however, like the condensation to small, compact clumps from larger gas clouds, should happen in a very similar manner at high redshift as we observe them today in the local universe. 


\subsection{Some notes on the sample selection}

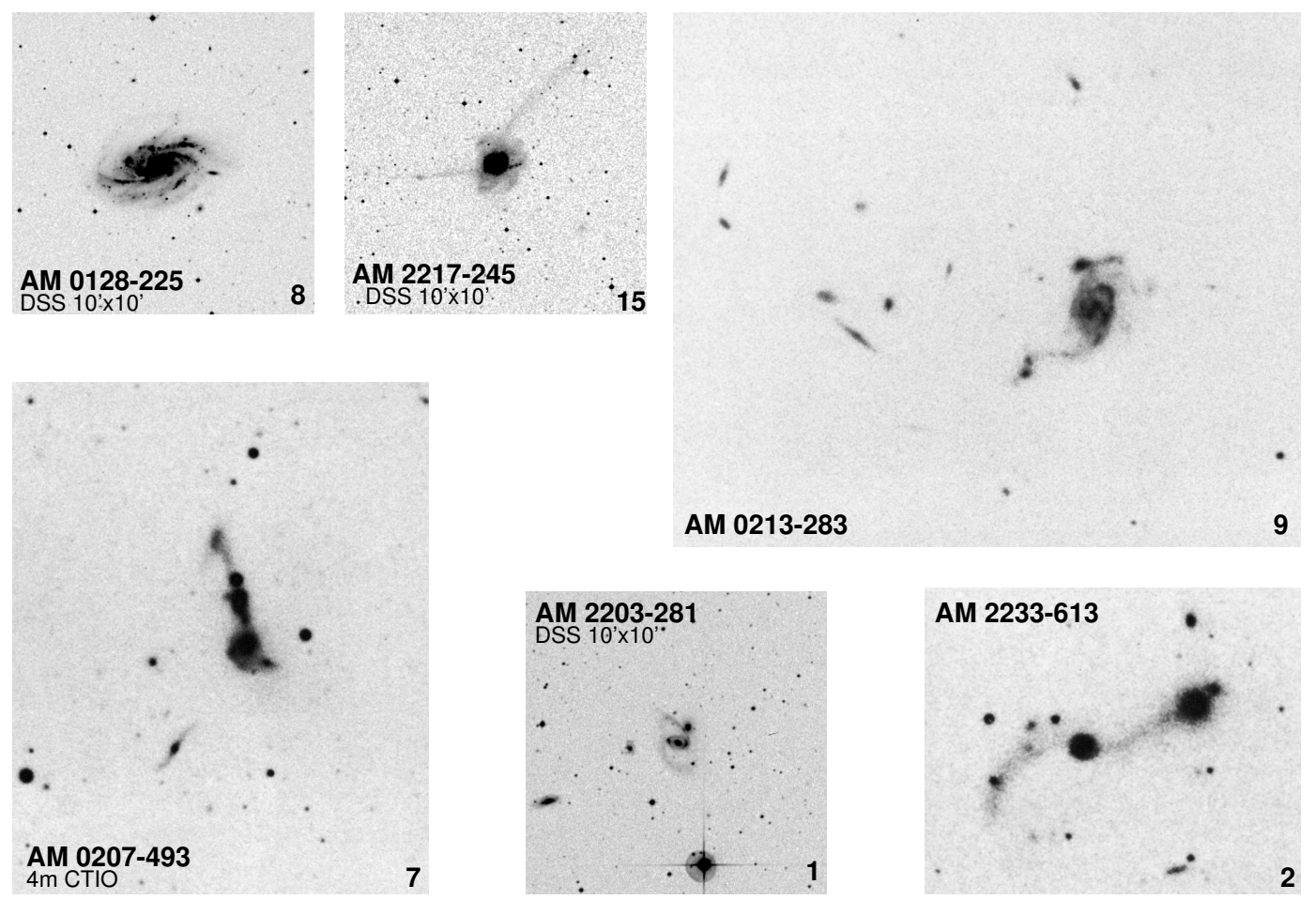

Figure 1: Some examples of galaxies from the AM catalog. AM 2217-245 is more widely knows as NGC 7252 or "Atoms for Peace". The images marked DSS are taken from the Digital Sky Survey and have a size of $10^{\prime} \times 10^{\prime}$, the other images are scanned from Vol. II of the Arp \& Madore catalog.

The sample of interacting and merging galaxies discussed in this thesis is taken from the catalog of Arp \& Madore (1987). Their Catalogue of Southern Peculiar Galaxies and Associations consists of two volumes. Vol. I lists 6445 extragalactic and galactic objects on the southern sky together with short characterizing descriptions sorted into 25 different categories. In the short description of each object, some key words are contained which hint to e.g. long tidal tails or other features interesting for the purpose of finding TDGs. Such key words are: "condensation", "jet", "integral spiral", "knotty", "loop", and of course "tail". Vol. II then shows reproductions of photographic plates for the best and most interesting examples from these categories.

Six objects pictured in Vol. II of the catalog are reproduced here as Fig. 1 to show the variety of galaxy types in the catalog. None of these objects were selected for our sample, but some contain knots which could be TDGs. In the Figure, the categories 
are: 1 Galaxies with interacting companion(s), 2 Interacting doubles (galaxies of comparable size), 7 Galaxies with (linear) jets, 8 Galaxies with apparent companion(s), 9 M51-types (companions at end of spiral arm), and 15 Galaxies with tails, loops of material or debris.

One goal of the analysis of our sample was to find out how many TDGs are created on average during galaxy interactions. In principle, this would require a randomly selected sample of interacting galaxies. To define such a neutral sample of interacting galaxies is not easy. How disturbed do they have to be in order to be called interacting? How long and how dense do the tidal tails have to be to form TDGs? How far is the galaxy away from us, and what is the true distance to the apparent companion? It is not possible to randomly select galaxies because then one may end up without any TDG candidates in their surroundings and without any conclusions on the properties of TDGs. But instead of just selecting the mergers with long tails and one conspicuous knot, a compromise was found by selecting interacting galaxies of chaotic shape but with probable knots in the tidal features were for the first imaging. It should also be pointed out that very little had been previously known about the objects in the final sample. Before the investigation of Arp 105 and Arp 245 started, color information was already available from the work of Schombert et al. (1990), and the bright tidal tails left no room for doubts about the association of the TDG candidates with the main interacting system. On the contrary, for most objects discussed in this thesis, even such basic information like redshifts and hence distances and sizes of these galaxies was unknown.

Images of the systems of the final sample are shown in Paper I (Fig. 3 to 22) and in Paper II (Fig. 36 to 48). A "true" color image of AM 1353-272 is displayed in Paper V (Fig. 58 on page 135).

\section{Thesis Outline}

A first step is to try and find a sample of TDGs for which ages, burst strengths, masses, and kinematic properties can be investigated in detail. Optical observations of the selected interacting galaxies obtained with the ESO NTT are presented in Paper I of this thesis. We create evolutionary synthesis models for starbursts on top of a mixed-age stellar population to select our sample of promising candidates for TDGs among the numerous knots apparent in the tidal features, reject background objects, and get a first idea of the strengths of the star formation episode in these TDG candidates.

After this first photometric selection and analysis we integrated the possibility of spectral synthesis into the models. During this work, it became apparent that the $\mathrm{H} \alpha$ luminosity is not always a reliable tracer of the current star formation rate, as previously thought. This led to a more thorough analysis with new models for 
starbursts, in particular those with short timescales. We also investigated some other problems related to the determination of the correct current star formation rate in an extragalactic object. This discussion is not only relevant for the investigation of TDGs, but also for other types of dwarf galaxies as well as any other system or region with star formation rates changing on timescales shorter than $10^{7} \mathrm{yr}$. The paper is therefore included as a related topic in Paper IV after the three papers on the sample of interacting galaxies.

Spectroscopic follow-up observations of the sample of TDG candidates preselected from optical photometry were carried at the ESO 3.6m telescope and confirmed the association of of these TDG candidates with the main interacting system in all cases where a redshift could be measured from the spectra. Another motivation for the spectroscopy was to further restrict the input parameters for the photometric models, especially the metallicity, which is a critical parameter as demonstrated in Sect. 3 of Paper I. Additionally, these observations showed some strikingly large velocity gradients in some of the TDG candidates. All this is discussed in Paper II. We discuss our near-infrared observations obtained with the ESO NTT of the sample in Paper III. These were carried out to provide a longer wavelength range for the comparison with the evolutionary synthesis models. With these observations and the optical data on the systems, we are able to create optical-NIR spectral energy distributions of all TDG candidates. These allow to better constrain the model parameters, in particular the relative contribution of an older stellar component, and are necessary to derive the stellar mass of the knots.

One of the most interesting interacting galaxies of our sample, called "The Dentist's Chair" (AM 1353-272), was observed with the ESO VLT at higher spectral resolution. Along both tails in this system we found - for the first time - several knots with significant internal velocity gradients. The interpretation of these objects as currently forming TDGs with first signs of kinematical decoupling from the surrounding tidal structures which could provide a base for rotational motion is layed out as Paper V.

The summary (Part VI connects all the results from this thesis and tries to view the results from our sample in relation to the general properties of TDGs. Possible topics for future research following from the results of this thesis are presented in Sect. 3.

\section{References}

Arp, H., 1966, ApJS 14, 1

Arp, H., 1969, A\&A 3, 418 
Arp, H.C., Madore, B.F., 1987, A Catalogue of Southern Peculiar Galaxies and Associations, Cambridge University Press, Cambridge

Barnes, J.E., Hernquist, L., 1992, Nature 360, 715

Braine, J., Duc, P.-A., Lisenfeld, U., et al., 2001, $A \& A$

Braine, J., Lisenfeld, U., Duc, P.-A., Leon, S., 2000, Nature 403, 867

Brouillet, N., Henkel, C., Baudry, A., 1992, A\&A 262, L5

Contardo, G., Steinmetz, M., Fritze-von Alvensleben, U., 1998, ApJ 507, 497

Duc, P.-A., Brinks, E., Springel, V., et al., 2000, AJ 120, 1238

Duc, P.-A., Brinks, E., Wink, J.E., Mirabel, I.F., 1997, A\&A 326, 537

Duc, P.-A., Mirabel, I.F., 1994, A\&A 289, 83

Duc, P.-A., Mirabel, I.F., 1998, A\&A 333, 813

Duc, Pierre-Alain, 1995, Genèse de galaxies naines dans les systèmes en interaction., PhD Thesis, Université Paris VI

Ellis, R., Santos, M.R., Kneib, J., Kuijken, K., 2001, ApJL 560, L119

Elmegreen, B.G., Kaufman, M., Thomasson, M., 1993, ApJ 412, 90

Fritze-von Alvensleben, U., Krüger, H., Fricke, K.J., Loose, H.-H., 1989, A\&A 224, L1

Fritze-von Alvensleben, U., Möller, C.S., Duc, P.-A., 1998, in: Dwarf Galaxies and Cosmology (eds. T.X. Thuan, C. Balkowski, V. Cayatte, J. Trân Thanh Vân), Editions Frontières, Paris, 293

Hernquist, L., 1992, Nature 360, 105

Hibbard, J.E., Guhathakurta, P., van Gorkom, J.H., Schweizer, F., 1994, AJ 107, 67

Hibbard, J.E., Mihos, J.C., 1995, AJ 110, 140

Hibbard, J.E., van der Hulst, J.M., Barnes, J.E., Rich, R.M., 2001, AJ 122, 2969

Hu, E.M., Cowie, L.L., McMahon, R.G., et al., 2002, ApJL 568, L75

Hu, E.M., McMahon, R.G., Cowie, L.L., 1999, ApJL 522, L9 
Hubble, E.P., 1936, Realm of the Nebulae, Yale University Press

Huchra, J., 2001, A\&SSS 277, 621

Hunsberger, Sally D., 1998, Dwarf Galaxies and Their Formation: a Study in the Compact Group Environment, PhD Thesis, Pennsylvania State University

Hunsberger, S.D., Charlton, J.C., Zaritsky, D., 1996, ApJ 462, 50

Hunter, D.A., Hunsberger, S.D., Roye, E.W., 2000, ApJ 542, 137

Iglesias-Páramo, J., Vílchez, J.M., 2001, ApJ 550, 204

Izotov, Y.I., Thuan, T.X., 1999, ApJ 511, 639

Kroupa, P., 1997, New Astronomy 2, 139

Kroupa, P., 1998, in: Dynamics of Galaxies and Galactic Nuclei (eds. Wolfgang J. Duschl, Christian Einsel), 243

Kunth, D., Maurogordato, S., Vigroux, L., 1988, A\&A 204, 10

Legrand, F., 2000, $A \& A$ 354, 504

Loose, H., Thuan, T.X., 1986, ApJ 309, 59

Lynden-Bell, D., Lynden-Bell, R.M., 1995, MNRAS 275, 429

Mendes de Oliveira, C., Plana, H., Amram, P., Balkowski, C., Bolte, M., 2001, AJ 121,2524

Mirabel, I.F., Dottori, H., Lutz, D., 1992, A\&A 256, L19

Mirabel, I.F., Lutz, D., Maza, J., 1991, A\&A 243, 367

Nishiura, S., Shioya, Y., Murayama, T., et al., 2002, PASJ 54, 21

Okazaki, T., Taniguchi, Y., 2000, ApJ 543, 149

Papaderos, P., Izotov, Y.I., Thuan, T.X., et al., 2002, A\&A 393, 461

Papaderos, P., Noeske, K.G., Cairós, L.-M., Vílchez, J.M., Fricke, K.J., 2001, in: Dwarf Galaxies and their Environment (eds. K.S. de Boer, R.-J. Dettmar, U. Klein), 283-286

Schombert, J.M., Wallin, J.F., Struck-Marcell, C., 1990, AJ 99, 497 
Schweizer, F., 1978, in: Structure and Properties of Nearby Galaxies (eds. E.M. Berkhuisen, R. Wiebilinski), 279

Searle, L., Sargent, W.L.W., 1972, ApJ 173, 25

Stockton, A., 1972, ApJ 173, 247

Temporin, Sonia G., 2001, Observations and Modeling of an Ultracompact Group of Galaxies, PhD Thesis, Leopold-Franzens-Universität, Innsbruck

Tinsley, B.M., 1972, A\&A 20, 383

Tolstoy, E., Gallagher, J.S., Cole, A.A., et al., 1998, AJ 116, 1244

Tolstoy, E., Saha, A., 1996, ApJ 462, 672

Toomre, A., Toomre, J., 1972, ApJ 178, 623

Tosi, M., Greggio, L., Marconi, G., Focardi, P., 1991, AJ 102, 951

Vanzi, L., Hunt, L.K., Thuan, T.X., Izotov, Y.I., 2000, A\&A 363, 493

Vorontsov-Velyaminov, B.T., 1959, Atlas and Catalogue of Interacting Galaxies, Sternberg Institute, Moscow State University, Moscow

Weilbacher, P.M., Duc, P.-A., 2001, in: Dwarf Galaxies and their Environment (eds. K.S. de Boer, R.-J. Dettmar, U. Klein), 269-272

White, S.D.M., Rees, M.J., 1978, MNRAS 183, 341

Williams, R. E., Baum, S., Bergeron, L. E., et al., 2000, AJ 120, 2735

Williams, R.E., Blacker, B., Dickinson, M., et al., 1996, AJ 112, 1335

Yoshida, M., Taniguchi, Y., Murayama, T., 1994, PASJ 46, L195

Zwicky, F., 1956, Erg. d. exakt. Naturwiss. 29, 344 


\section{Paper I}

\section{Sample Selection: Optical Photometry and Models}

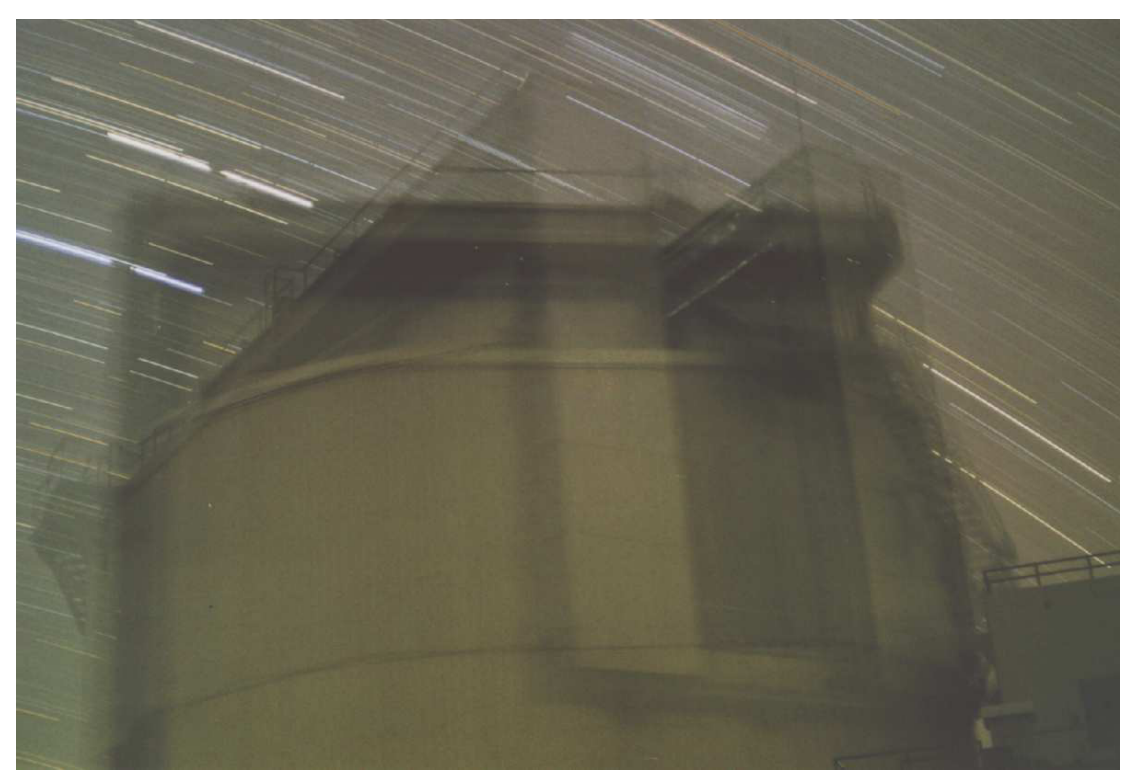



This paper was published May 2000 in

Astronomy \& Astrophysics, 358, 819-834.

\title{
Tidal dwarf candidates in a sample of interacting galaxies*
}

\author{
Peter M. Weilbacher ${ }^{1}$, P.-A. Duc ${ }^{2,3}$, U. Fritze-v.Alvensleben ${ }^{1}$, P. Martin ${ }^{4}$, and \\ K. J. Fricke ${ }^{1}$ \\ ${ }^{1}$ Universitätssternwarte, Geismarlandstr. 11, D-37083 Göttingen, Germany \\ ${ }^{2}$ CNRS URA 2052 and CEA, DSM, DAPNIA, Service d'Astrophysique, Centre d'Etudes de Saclay, \\ 91191 Gif-sur-Yvette Cedex, France \\ ${ }^{3}$ Institute of Astronomy, University of Cambridge, Madingley Road, Cambridge, CB3 0HA, UK \\ ${ }^{4}$ Canada-France-Hawaii Telescope, P.O. Box 1597, Kamuela, HI 96743, USA
}

\begin{abstract}
We present deep optical $B, V, R$ images of a sample of 10 interacting systems which were selected for their resemblance to disturbed galaxies at high redshift. Photometry is performed on knots in the tidal features of the galaxies. We calculate a grid of evolutionary synthesis models with two metallicities and various burst strengths for systems consisting of some fraction of the stellar population of a progenitor spiral plus starburst. By comparison with two-color diagrams we interpret the photometric data, select from a total of about 100 condensations 36 star-forming objects that are located in the tidal features and predict their further evolution. Being more luminous by 4 mag than normal $\mathrm{H}$ II regions we argue that these objects could be tidal dwarf galaxies or their progenitors, although they differ in number and mean luminosity from the already known tidal dwarf galaxies typically located at the end of tidal tails in nearby giant interacting systems. From comparison with our models we note that all objects show young burst ages. The young stellar component formed in these tidal dwarf candidates contributes up to $18 \%$ to the total stellar mass by the end of the starburst and dominates the optical luminosity. This may result in fading by up to 2.5 mag in $B$ during the next 200 Myrs after the burst. Key words: Galaxies: formation - Galaxies: interactions - Galaxies: photometry - Galaxies: evolution
\end{abstract}

*Based on observations collected at the European Southern Observatory, La Silla, Chile (ESO No 058.A-0260). 


\section{Introduction}

Most investigations of interacting and merging galaxies have essentially focussed on the phenomena occuring in their inner regions. However, for a few spectacular cases like the Antennae (Mirabel et al. 1991), Arp 105 (Duc \& Mirabel 1994, Duc et al. 1997) and NGC 7252 (Hibbard \& Mihos 1995) the enormous tidal tails were investigated in some detail. Massive condensations of stars and $\mathrm{H} \mathrm{I}$ were found in these tails, the so called Tidal Dwarf Galaxies (TDGs).

TDGs are characterized by a luminosity comparable with that of typical dwarf galaxies, but which span a range in oxygen abundance of $8.3 \lesssim 12+\log (O / H) \lesssim$ 8.6 equivalent to metallicities of $4 \cdot 10^{-3} \lesssim Z_{\mathrm{TDG}} \lesssim 8 \cdot 10^{-3}$ with a mean of $Z \approx 7 \cdot 10^{-3} \approx Z_{\odot} / 2$. . $^{1}$ Metallicities of TDGs are therefore higher than those of other dwarf galaxies with comparable luminosity (Duc \& Mirabel 1997, 1998). TDGs have blue colors as a result of an active starburst. Most known TDGs have high H I masses from which total masses of the order of $10^{9} \mathrm{M}_{\odot}$ have been estimated. They are gravitationally bound and in a few interacting systems evidence for their kinematical independence has been found (e.g. Duc et al. 1997, Duc \& Mirabel 1998). These systems together with the strong increase of the galaxy merger rate with redshift gave rise to speculations about a possible contribution of TDGs to the faint blue galaxy excess at high redshift (Fritze-von Alvensleben et al. 1998). Barnes \& Hernquist (1992) observed the formation of TDGs in their numerical simulations. Their 3D N-body/SPH-code created several massive bound condensations along the tidal tails. At first, their mass is dominated by the stellar component. Later on, gas from the tail might fall into these condensations. Elmegreen et al. (1993) proposed a different scenario. They have used a 2D N-body code including dissipation for the gas component in which gaseous condensations form first.

Observations in most cases show both components, neutral gas and stars, along the tails as well as in their condensations. In an optically indentified condensation, determination of the relative fraction of young stars - born in situ from the collapse of tidally extracted $\mathrm{H} \mathrm{I}$ - to old stars - pulled out from the progenitor disk(s) - might constrain the above mentioned numerical models. Because the systems studied here are too distant to be resolved into stars, estimating these relative stellar fractions can only be done from multi-band integrated photometric measurements in comparison with an evolutionary synthesis model. Photometric work on the tidal features of several classical interacting systems has been carried out by Schombert et al. (1990). Studies by Appleton \& Marston (1997) and Bransford et al. (1998) concentrated on ring galaxies. Deeg et al. (1998) have systematically catalogued all faint non-stellar objects around disturbed galaxies. Part of them might be TDG candidates.

\footnotetext{
${ }^{1}$ For metallicity we use the notation of $Z$ (as the proportion of heavy elements given in the Geneva stellar tracks which our models are based on) throughout this paper and give a comparison with the solar value $Z_{\odot}=0.018$ where appropriate.
} 
These previous investigations have focused on disk-disk interacting systems with a well-understood morphology, e.g. well defined tails. However locally the majority of disturbed galaxy systems, for instance in the Catalogue of Southern Peculiar Galaxies (Arp \& Madore 1987), do not have long tails. In particular disk-spheroid collisions and 3-body systems leading to chaotic morphologies are quite common. HST deep surveys with the WFPC2 show that the percentage of disturbed (i.e. interacting, merging or peculiar) galaxies has been much higher in the early universe than today (van den Bergh et al. 1996). NICMOS observations in the near-infrared have shown that these disturbances are not only due to irregularities in the distribution of star forming regions, but reflect real morphological features of interactions (Conselice et al. 1998). These galaxies at high redshift often do not show long tidal tails either because they are absent or because they have too low a surface brightness. Examining a local sample which resembles these high redshift interacting galaxies is therefore important to learn about their evolution.

In this paper we extend previous investigations of a few individual systems to deep optical photometry of a sample of peculiar galaxies. The objects from our sample mostly do not possess prominent tidal tails, but definitely show signs of merging, as e.g. multiple nuclei or disturbed morphology and nearby companions. The sample is extracted from the Catalogue of Southern Peculiar Galaxies (Arp \& Madore 1987). It includes disk-disk, disk-elliptical, dwarf-dwarf and 3-body systems observed at different stages of the interaction.

Spectrophotometric evolutionary synthesis models have been used for various applications of normal galaxy evolution. More recently starburst events were modeled as well. These models assume that the standard star-formation rate (SFR) of an underlying galaxy is increased to simulate a starburst (Fritze-von Alvensleben \& Gerhard 1994a, Leitherer \& Heckman 1995). This technique has been applied to reproduce the star formation history of various classes of objects from merging galaxies (Fritze-von Alvensleben \& Gerhard 1994b) to blue compact galaxies (Krüger et al. 1995).

We take an approach similar to the latter and compute a grid of models specifically aimed to describe the properties found in previous investigations of TDGs. We do not use solar-abundance tracks in our models but instead two more realistic metallicities in the range expected for TDGs $\left(Z=1 \cdot 10^{-3}, 8 \cdot 10^{-3}\right)$. As a primary goal, these models are used to select candidate TDGs or TDG progenitors. Based on their colors only, background objects can be excluded not only with statistical methods but also one by one if they disagree with any kind of TDG model. Furthermore, this technique allows to derive the present ratios of old to young stellar mass in these TDG candidates, to predict its evolution and hence to constrain the above-mentioned formation scenarios of TDGs. Obviously spectrophotometric data is required to confirm the photometric results. 
Table 1: Observation log

\begin{tabular}{rccccrr}
\hline Field & Object & Observing Date & Seeing & \multicolumn{3}{c}{ total Exposure Time [s] } \\
No. & & & $\left.{ }^{\prime \prime}\right]$ & $B$ & \multicolumn{1}{c}{$V$} & \multicolumn{1}{c}{$R$} \\
\hline 1 & AM 0529-565 & $02 . / 03.03 .97$ & 0.8 & 2400 & 1800 & 1800 \\
2 & AM 0537-292 & 28.02 .97 & 0.9 & 1200 & 900 & 900 \\
3 & AM 0547-244 & 01.03 .97 & 1.1 & 1200 & 900 & 900 \\
4 & AM 0607-444 & 03.03 .97 & 0.8 & 1200 & 900 & 900 \\
5 & AM 0642-645 & 08.04 .97 & 1.3 & 1200 & 900 & 900 \\
6 & AM 0748-665 & 10.04 .96 & & & & \\
7 & AM 1054-325 & $16.03 . / 03.04 .97$ & 1.1 & 3600 & 3000 & 3000 \\
8 & AM 1208-273 & 03.04 .97 & 0.8 & 1200 & 900 & 900 \\
9 & AM 1325-292 & 03.04 .97 & 0.9 & 1200 & 900 & 900 \\
10 & AM 1353-272 & $15 . / 16.03 .97$ & 0.8 & 2400 & 1200 & 1200 \\
\hline
\end{tabular}

The structure of the paper is as follows. First we present our data reduction and analysis techniques (Sect. 2). We describe in Sect. 3 the evolutionary synthesis models and the parameters used to interpret the photometric data. In Sect. 4 we present our sample, compare with the model grid and comment on individual features of the interacting systems. In Sect. 5 we discuss more general results and in particular compare the tidal objects identified in our sample with those produced in numerical models and with the few typical TDGs studied so far.

\section{Observations and data reduction}

\subsection{Data and Calibration}

Optical $B, V, R$ images of 10 galaxies were obtained in visitor mode on April 10th, 1996 and in service mode from February to April 1997 using SUSI on the NTT. The SUSI camera has a field of view of $2 ! 2 \times 2 ! 2$ and a pixel scale of $0 . \prime 13$. The objects of our sample with observing dates, seeing conditions and total exposure times are given in Table 1.

The data reduction was performed in IRAF using the ccdred package with the classical procedures of bias-subtraction and flat-fielding. In order to correct the sky background for some residuals we constructed illumination frames by modeling the background fitted at positions not contaminated by object light. Cosmic ray hits were removed using cosmicrays. The images in all filters were then registered and PSF-matched using several reference stars. Foreground stars were, when possible, deleted and replaced by a background fit using the imedit task. 
Standard stars from the fields of Landolt (1992) have been used to derive the zeropoints and estimate the color terms. The extinction coefficients were taken from the La Silla extinction database provided by the Geneva group (e.g. Burki et al. 1995). With this data we could derive a photometric calibration accurate to about 0.04 mag for most targets. One should note that most of the observations were obtained during the refurbishment of the NTT and that the standard stars have not always been observed during the same night as the science targets. Therefore additional systematic calibration errors cannot be excluded; however, our zeropoints and color terms appear to be very stable from one night to the other. Their variations are smaller than 0.01 mag i.e. smaller than the given errors. Except for a few cases for which the extinction given by the Geneva group has large uncertainties, high calibration errors

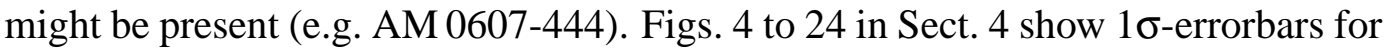
the systematic error for each field due to uncertain calibration and individual errors for each object due to photon statistics and background noise.

We also obtained longslit-spectra of AM 0529-565, AM 1325-292, and AM 1353272 using EMMI at the NTT in May 1998. The reduction was performed in the standard way and the spectra were used to measure the redshift. Further details will be given in a forthcoming paper.

\subsection{Aperture photometry}

As the features of interest within the tidal debris are irregular in shape and located in regions with high and irregular background, traditional photometry with circular apertures was not appropriate since it would have included too much flux from the surrounding tidal features. We therefore developed a reproducible technique to analyze these knots using polygonal apertures and used circular apertures only for objects far away from the main body of the interacting system.

The $V$-band intensity scale of each image was transformed to calibrated surface brightness and contour plots were created of the interesting regions of each frame. The objects to measure were selected from the most prominent peaks in surface brightness. Polygonal apertures were then defined following the faintest contour which still allows separating the knot from surrounding tidal features. The surface brightness level $\mu_{V}$ of the aperture was therefore different for each knot and is given in Tables 5 to $14^{2}$. The sky flux was individually measured at several positions nearby each knot, carefully avoiding the tidal features, and averaged. We are interested in both the young and the old stellar population which the (stellar) tail consists of, because we explicitly model the old population in our evolutionary synthesis models. Hence we only subtract the sky background but not the tidal tail from the object flux. The photometry was finally performed using the IRAF polyphot

\footnotetext{
${ }^{2}$ Tables 5 to 14 are available only in electronic form.
} 
task with the same polygonal aperture for all photometric bands. Tables 5 to 14 give the complete photometric results. Column 1 gives the identification, column 2 the surface brightness $\mu_{V}$ of the polygonal aperture. Columns 3 to 5 show the apparent magnitudes in $B, V$ and $R$, while columns 6 to 8 present the optical colors $B-V$, $V-R$ and $B-R$. Columns 9 to 11 show the photometric errors of the measurements in $B, V$ and $R$. All given magnitudes and colors are corrected for galactic extinction, using the $A_{B}$ values of Burstein \& Heiles (1982) and extrapolating to $V$ and $R$ with the ratios of Savage \& Mathis (1979).

\section{Model}

\subsection{Modelling the old population}

Spectrophotometric and chemical evolutionary synthesis models are used to interpret the photometric data. Our code is based on the work of Krüger (1992) which was originally used to model BCDGs (Krüger et al. 1995). To include the underlying old component in our model, it starts with a gas cloud of primordial metallicity and follows the evolution of ISM abundances and spectrophotometric properties of the stellar population of an undisturbed galaxy until the starburst induced by the interaction occurs. The model includes the two basic parameters, star formation rate (SFR) $\psi(t)$ and initial mass function (IMF). We use SFRs proportional to the gas to total mass ratio for Sb and Sc models and a constant SFR for Sd with efficiencies appropriate for the respective galaxy types (see e.g. Fritze-von Alvensleben \& Gerhard 1994a).

As our observations cannot constrain the IMF, we use the IMF from Scalo (1986) in the mass range 0.15 to $120 \mathrm{M}_{\odot}$ and, for simplicity, assume the same IMF for the progenitor spiral and the starburst. To cover a Hubble time of evolution for the undisturbed progenitor spiral and have a good time resolution during and after the burst, we use a variable timestep as outlined by Krüger (1992).

\subsection{Input physics}

The model uses Geneva stellar evolutionary tracks (Schaller et al. 1992, Charbonnel et al. 1993, Schaerer et al. 1993a,b, Charbonnel et al. 1996). We present models for two different metallicities $Z_{1}=1 \cdot 10^{-3} \approx Z_{\odot} / 18$ and $Z_{3}=8 \cdot 10^{-3} \approx Z_{\odot} / 2.3$.

As shown by Krüger et al. (1995) the broadband colors are dominated by nebular line and continuum emission in the early phases of a strong burst. We therefore include nebular emission in our models using the compilation from Schaerer \& de 
Koter (1997) to calculate the number of Lyman continuum photons emitted per second $N_{\text {Lyc }}$ of our stellar population as a function of time. With the standard formula

$$
F(\mathrm{H} \beta)=4.757 \cdot 10^{-13} f N_{\mathrm{Lyc}} \operatorname{erg~s}^{-1}
$$

we obtain the $\mathrm{H} \beta$-flux. Fluxes of the other hydrogen lines are computed from their line ratios relative to $\mathrm{H} \beta . \quad f$ is the proportion of Lyman continuum photons not absorbed in dust. We take $f=1.0$ for $Z_{1}$ and $f=0.7$ for $Z_{3}$.

To include other typical lines of star-forming regions we use the observed line ratios of 27 lines in the wavelength range from 1335 to $10330 \AA$ from Izotov et al. (1994) for low metallicity $\left(Z_{1}\right)$ and theoretical line ratios from Stasińska (1984) for medium metallicity $\left(Z_{3}\right)$ models.

We chose to use the metallicities $Z_{1}$ and $Z_{3}$ given above ${ }^{3}$. In the context of comparing our models to observed objects these metallicities should not be regarded as fixed values, but more as metallicity ranges, $Z_{1}$ as $0.001<Z<0.006$ and $Z_{3}$ as $0.006<Z<0.012$.

\subsection{TDG models}

To model both the star-forming young and the old component of a TDG with our 1-zone model, we let a typical part of a disk galaxy evolve with its appropriate SFR over 13 Gyrs. After 13 Gyrs a starburst is assumed to occur (triggered by an interaction) in this part of the model galaxy assumed to form a TDG. In the burst the SFR is set to a maximum value $\psi_{0}$ and decreases exponentially with a timescale $\tau_{\mathrm{B}}$.

The time of the starburst is inferred from a rough age of present-day galaxies of 14 Gyr in a reasonable cosmological model. We look back roughly 1 Gyr, the assumed epoch for the starburst following an interaction. The model is followed for another 3 Gyrs after the burst. The galaxy age at the onset of the burst does not significantly change the color evolution during or after the burst.

According to previous observations the model with metallicity of $Z_{3}$ should a priori best apply to TDGs. In the dynamical formation scenarios of Barnes \& Hernquist (1992) and Elmegreen et al. (1993), TDGs are predicted to form out of material from the outer parts of the progenitor or from the progenitor galaxy disks. This material in nearby galaxies has a metallicity of $Z \approx 7 \cdot 10^{-3} \approx Z_{3}$ (Zaritsky et al. 1994, Ferguson et al. 1998). Indeed, spectroscopy of TDGs confirms this mean metallicity (Duc \& Mirabel 1997, 1998). However, the precise metallicity expected for a particular condensation depends on the type and luminosity of the progenitor galaxy late type and low luminosity spirals being more metal poor on average than earlier

\footnotetext{
${ }^{3}$ Another possible metallicity given in the Geneva stellar tracks $\left(Z_{2}=4 \cdot 10^{-3}\right)$ is not used in our models because no emission line ratios are available for it.
} 
Table 2: Set of model parameters.

\begin{tabular}{rcrccc}
\hline Run & $\tau_{\mathrm{B}}$ & \multicolumn{2}{c}{$\psi_{0}$} & $b$ & \multicolumn{2}{c}{$L_{\text {young }} / L_{\text {tot }}$} \\
ID & {$[\mathrm{yr}]$} & {$\left[\mathrm{M}_{\odot} \mathrm{yr}^{-1}\right]$} & & $B$ & $R$ \\
\hline 1 & $5 \cdot 10^{5}$ & 100 & 0.01 & 0.56 & 0.37 \\
2 & $5 \cdot 10^{5}$ & 500 & 0.05 & 0.87 & 0.75 \\
3 & $5 \cdot 10^{5}$ & 1000 & 0.10 & 0.93 & 0.85 \\
\hline 4 & $1 \cdot 10^{6}$ & 100 & 0.02 & 0.69 & 0.49 \\
5 & $1 \cdot 10^{6}$ & 500 & 0.09 & 0.92 & 0.83 \\
6 & $1 \cdot 10^{6}$ & 1000 & 0.18 & 0.96 & 0.91 \\
\hline 7 & $5 \cdot 10^{6}$ & 50 & 0.04 & 0.76 & 0.58 \\
\hline
\end{tabular}

type or higher luminosity spirals - and on the region of the parent galaxies where this particular material is torn out from, since galaxies tend to have negative abundance gradients in stars and the gas with increasing radius. Both at higher redshift in general and in the local universe in those cases where dwarf or very low metallicity galaxies are involved in the encounter, TDGs with lower metallicity might also form. However, the recycled nature of TDGs precludes metallicities lower than about $1 / 20$ solar. Models with $Z_{1}$ predict that they should populate distinctly different regions in optical color-color diagrams, i.e. have very blue $V-R$ at moderately blue $B-V$. Models with $Z_{1}$ have been applied in those cases.

To produce the various proportions of old and young populations observed in TDGs, the maximum SFR and the timescale of the burst $\tau_{\mathrm{B}}$ are varied. The resulting burst strength $b$ is computed as the ratio of the mass of stars formed during the burst and the total mass of stars ever formed in the galaxy. Our model grid is presented in Table 2. Column 1 gives a code for each model, which is used in the plots in Sect. 4 together with the metallicity. Columns 2 and 3 give the max. SFR and decay time of the burst and column 4 the burst strength. Columns 5 and 6 give the relative contributions of the starburst component to the luminosities in the $B$ and $R$ bands.

\subsection{Model application and limitations}

We discuss here the application and limitations of our grid of models to derive the properties of the observed condensations in the tidal features. Fig. 2a details the track in a $B-V$ vs. $V-R$ two-color diagram for one given burst-model and various assumptions for the underlying parent galaxy. The color evolution is plotted from the start of the burst at 13 Gyrs (underlying galaxy Sd) when a subpopulation of the progenitor spiral is expelled into a tidal tail and begins to form a TDG. With the burst, the colors become bluer, at first most noticeable in $V-R$ and then in $B-V$ when the starburst begins to fade. In the two-color diagram this color evolution 

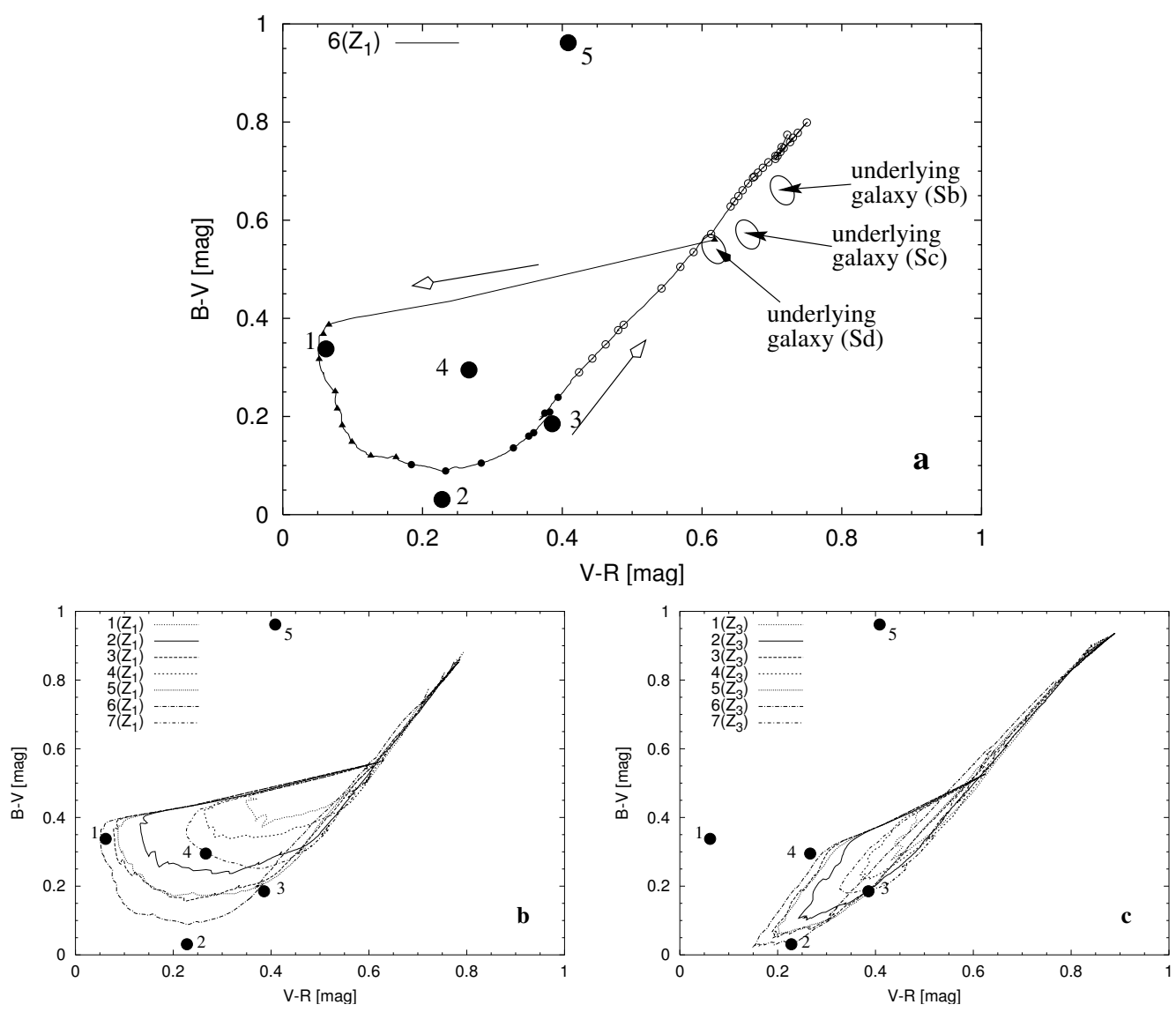

Figure 2: Examples of model curves in a $B-V$ vs. $V-R$ diagram for various burst parameters. Five "data points" are shown as examples, see discussion in the text. a shows the strongest low metallicity burst model with tick marks indicating the burst age: The triangles are separated by $1 \mathrm{Myr}$ starting at the onset of the burst, the filled circles are separated by 10 Myrs, and the open circles by 100 Myrs. b shows all low metallicity $\left(Z_{1}\right)$ burst loops and $\mathbf{c}$ all medium metallicity bursts.

produces a loop which is followed counterclockwise. While the color evolution is very rapid at the beginning of the burst, it slows down considerably as the burst ages. The type of the underlying galaxy and hence its star formation history slightly affect the starting point of the loop but do not significantly change its shape. Due to lack of detailed knowledge of the spectral type of the undisturbed progenitor we choose to uniformly model the old population with a constant SFR.

Figs. $2 \mathbf{b}$ and $\mathbf{c}$ display the curves of all models that have been probed for the two metallicities $Z_{1}$ and $Z_{3}$, respectively. It can be seen that a higher initial burst SFR $\psi_{0}$ and hence a higher burst strength $b$ produces a larger loop. The different shapes of 
the loops for models with equal strength but different metallicities is mainly caused by the variing fluxes of the emission lines which strongly contribute to the optical broad band colors during the burst (Krüger 1992).

The dots plotted in Fig. 2 show five representative artificial data points chosen to demonstrate in howfar the various properties of a burst can be disentangled using two colors only. Data point " 1 " lies in a region in the two-color plane which can only be reached by a low metallicity model with high burst strength $(b>0.1)$ and young burst age (about 2.5 Myrs). Data point " 2 " needs a high burst strength $(b>$ 0.1 ) and medium burst age (around 20 Myrs), whatever the metallicity, the medium metallicity model providing a better match. Data point " 3 " is more ambiguous with respect to the metallicity but clearly needs a much higher age (above $80 \mathrm{Myrs}$ ) and a medium to strong burst $(b>0.05)$. Depending on the metallicity data point " 4 " can be either an intermediate strength burst (low $Z, 0.02<b<0.05$, young age $\approx 1$ Myr) or strong burst (for medium $Z, b>0.10$, age $\approx 7$ Myrs). Additional colors or spectroscopy are needed to resolve the ambiguity. Data point " 5 " does not agree at all with any of our models. Its colors are indicative of a redshifted background galaxy (see Sect. 5.1 and Möller et al. in prep.).

In Sect. 4 we compare the photometry of the star-forming knots lying in or close to the tidal features of our interacting galaxies with predictions of various TDG models. The primary goal is to isolate objects consistent with a TDG scenario from background sources. A metallicity of $Z_{3}$ as observed for TDGs was a priori selected unless other indicators (a metallicity measurement from spectroscopic data, the lumosity class of the parent galaxy, or colors that clearly indicate a low metallicity model like data point " 1 ") favor a $Z_{1}$ model. The burst parameters were determined by visual comparison of the position of the data points to the model curves. The model with the smallest deviation from an observed data point was selected as "most likely" model from our grid. We can also assess which other models represent a reasonable match within the observational uncertainties and from that derive likely ranges for the burst age and strength of any tidal object (see Table 4). Given the individual and systematic photometric errors and the two-dimensional nature of the data, a numerical fitting routine did not seem to be warranted.

As the modelling of the undisturbed galaxy is created for an average late-type galaxy the colors of the nuclei or the parent galaxies plotted in Sect. 4 may have strongly differing colors (as in the case of AM 1325-292, see Fig. 21). This does not affect the validity of our models with respect to the TDG candidates.

It should be noted that the comparison of observation with models only relies on the two colors $B-V$ and $V-R$. To account for the effect of dust obscuration on the colors, a larger set of photometric data including the NIR regime or spectral information would be needed. In the following, we neglect any internal extinction. The effect of $A_{B}=0.5 \mathrm{mag}$ absorption is indicated by an arrow in the plots in Sect. 4. A significant extinction (of e.g. $A_{B}=0.5 \mathrm{mag}$ or more) would therefore result in un- 
Table 3: Properties of the parent galaxies.

\begin{tabular}{lcccl}
\hline Name & $\begin{array}{c}\text { Velocity } \\
{\left[\mathrm{km} \mathrm{s}^{-1}\right]}\end{array}$ & $\begin{array}{c}B \\
{[\mathrm{mag}]}\end{array}$ & $\begin{array}{c}M_{B}{ }^{1} \\
{[\mathrm{mag}]}\end{array}$ & Morphology \\
\hline AM 0529-565 A & $4420^{5}$ & 15.83 & -18.3 & Merger \\
AM 0529-565 D & $4645^{5}$ & 18.46 & -15.4 & dI \\
AM 0537-292 A & - & 14.97 & - & Sb? \\
AM 0537-292 B & - & 16.56 & - & E \\
AM 0547-244 A & $13164^{3}$ & 15.62 & -20.6 & S pec \\
AM 0547-244 B & - & 17.10 & - & S? \\
AM 0607-444 A & - & 15.36 & - & Sc:pec \\
AM 0642-645 A & - & 16.44 & - & Pec \\
AM 0748-665 A & - & 18.01 & - & S \\
AM 0748-665 B & - & 16.37 & - & E \\
AM 1054-325 A & $3795^{4}$ & 14.55 & -19.0 & Sm \\
AM 1054-325 B & - & 15.31 & - & S pec \\
AM 1208-273 A & $14778^{3}$ & 15.62 & -20.9 & Sc \\
AM 1208-273 B & $12433^{3}$ & 16.28 & -19.8 & Pec \\
AM 1325-292 A & $4598^{5}$ & 13.90 & -19.9 & SB $(s) b^{2}$ \\
AM 1325-292 B & $4431^{5}$ & 13.43 & -20.4 & E1 pec \\
AM 1353-272 A & $11791^{5,6}$ & 15.77 & -20.3 & Sc:pec \\
AM 1353-272 B & $12145^{5,6}$ & 17.96 & -18.1 & S pec \\
\hline
\end{tabular}

${ }^{1}$ We use $H_{0}=75 \mathrm{~km} \mathrm{~s}^{-1}$ throughout this paper

${ }^{2}$ The morphological type was taken from NED

${ }^{3}$ from Donzelli \& Pastoriza (1997)

${ }^{4}$ from Sekiguchi \& Wolstencroft (1993)

${ }^{5}$ from Weilbacher et al. 2000 in prep.

${ }^{6}$ these values are reversed like in the original publication; correct are $V_{\mathrm{A}}=12145 \mathrm{~km} \mathrm{~s}^{-1}$ and $V_{\mathrm{B}}=11791 \mathrm{~km} \mathrm{~s}^{-1}$.

derestimating the burst strength, but would not change the final conclusions drawn from the model.

\section{Results}

Figures 3 to 22 present surface brightness images of all the systems in our sample and the two-color diagrams for comparison with the evolutionary synthesis models. The objects of interest are identified on the charts and the plots. The principal objects - those that most probably pre-existed the interaction - have been labeled with capital letters, while the smaller features - those that are TDG candidates have small letters. An overview of the properties of all parent galaxies is given in 


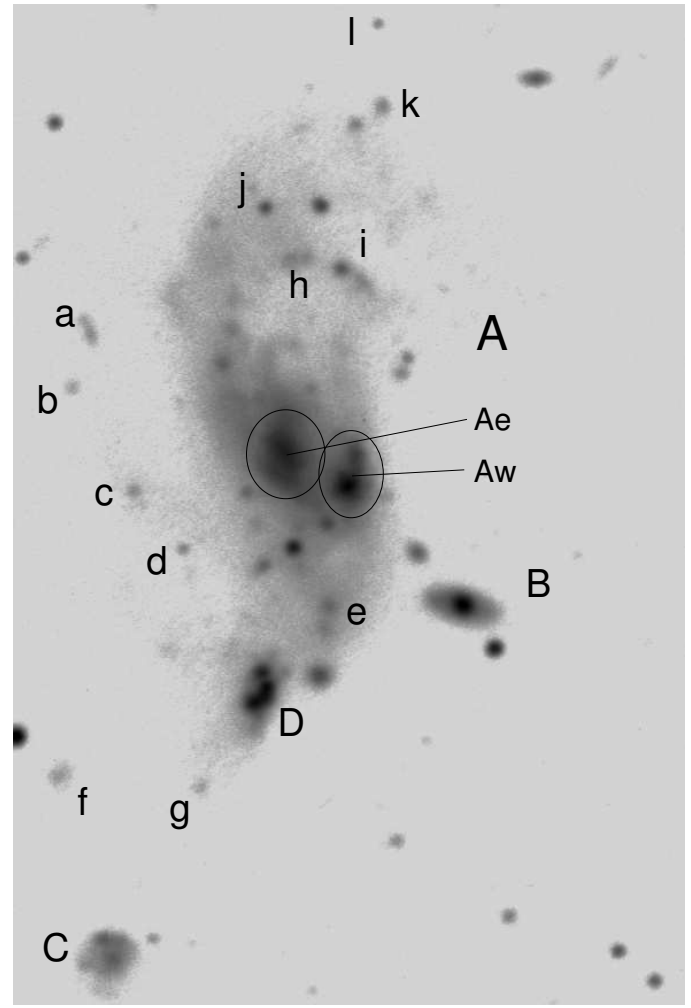

Figure 3: Identification chart of field 1 around AM 0529-565.

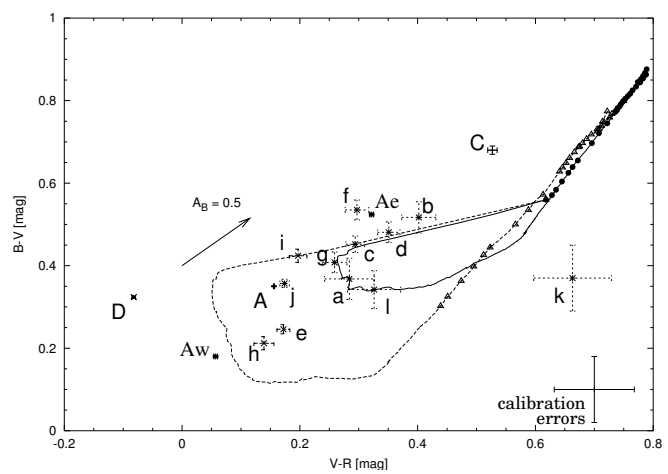

Figure 4: Plot of the objects in field 1 of AM 0529-565. The models used are $4\left(Z_{1}\right)$ (solid, $b=0.02$ ) and $6\left(Z_{1}\right)$ (dashed, $b=0.18$ ).

Table 3. The properties of the objects for which we have infered a tidal origin and are good candidates for TDGs or their progenitors are listed in Table 4. The model curves shown on the diagrams are those representing a maximum number of data points.

\subsection{AM 0529-565}

The image of this system at a redshift of $V_{\mathrm{A}}=4420 \mathrm{~km} \mathrm{~s}^{-1}$ is shown in Fig. 3 . The principal galaxy, "A", has an integrated absolute blue magnitude typical of a Magellanic Irregular galaxy. However the presence of a double nucleus and of diffuse tidal tails are characteristics of mergers. The eastern nucleus "Ae" has redder colors than the western one "Aw" which is currently in a starburst phase (see Fig. 4). " $\mathrm{B}$ " is an elliptical galaxy. Given its colors, it is probably a background object with an estimated redshift of $\approx 0.2$ (Möller et al. in prep.). "C" is a peculiar low surface brightness (LSB) galaxy of unknown redshift. "D" is a blue dwarf galaxy associated with "A" at a relative velocity of $V_{\text {rel }}=220 \mathrm{~km} \mathrm{~s}^{-1}$. Several diffuse knots can be 


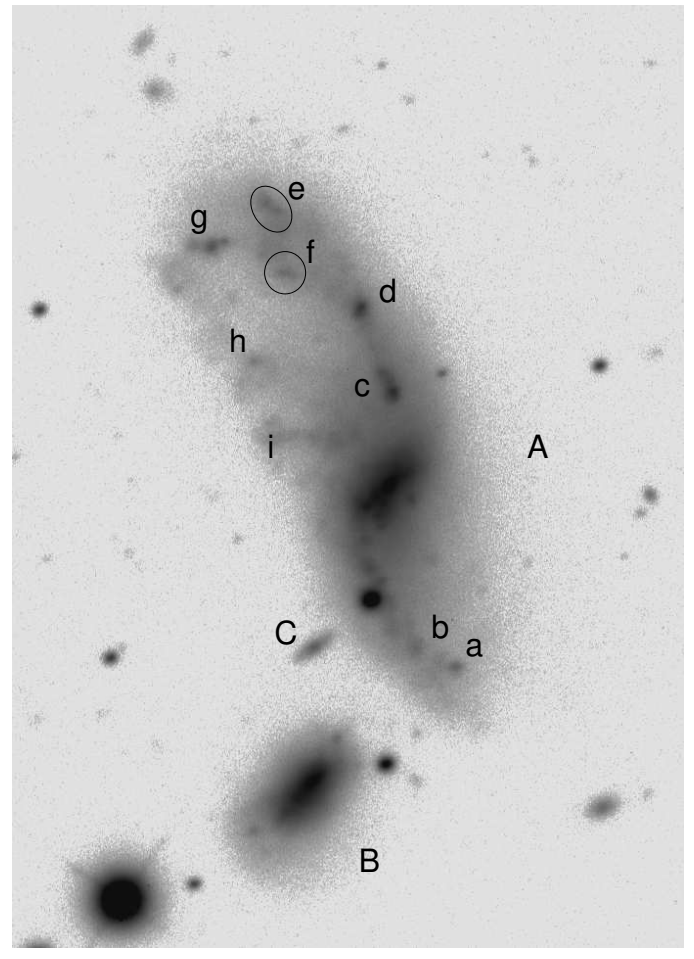

Figure 5: Identification chart of field 2 around AM 0537-292.

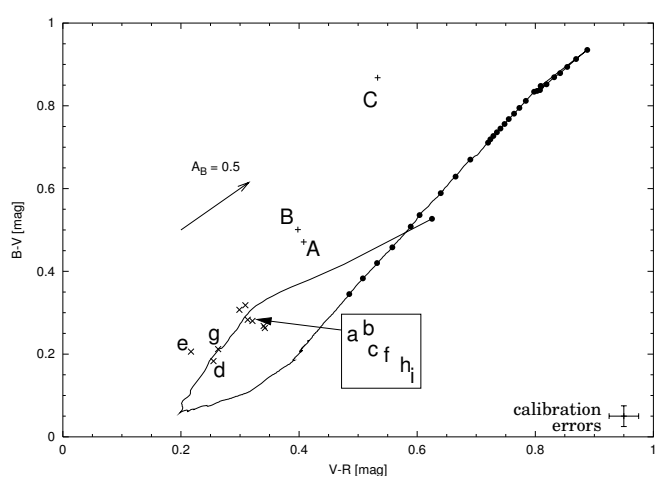

Figure 6: Plot of the objects in field 2 of AM 0537-292. The model used is $5\left(Z_{3}\right)$ (solid) with $b=0.09$.

seen around "A"; they are designated with "a" to "l". "a" seems to be detached from the eastern tidal tail.

From preliminary spectroscopic test observations of AM 0529-565 (Weilbacher et al. 2000, in prep.), we could estimate an oxygen abundance of $12+\log (O / H)=8.1$ (equivalent to $Z=0.003$ ) for "e" and "h" and a very $\operatorname{low} 12+\log (O / H)=7.6$ (equiv. to $Z=0.0008$ ) for object " $D$ ". Because of its much smaller metallicity, " $D$ " is most probably a pre-existing galaxy currently in interaction with " $A$ ".

Fig. 4 shows that the colors of most of the knots in the tidal debris are consistent with TDG burst models with low metallicity $\left(Z_{1}\right)$ and burst strength in the range $0.02<b<0.18$.

\subsection{AM 0537-292}

The image of this system also known as MCG-5-14-9 is shown in Fig. 5. "A" is a strongly disturbed galaxy with tidal tails and a central bar. " $\mathrm{B}$ " has the morphology of an E5 galaxy in the central part, but the contours get distorted further out. The overall color of both galaxies is strongly dominated by the light of the nuclei. "C" 


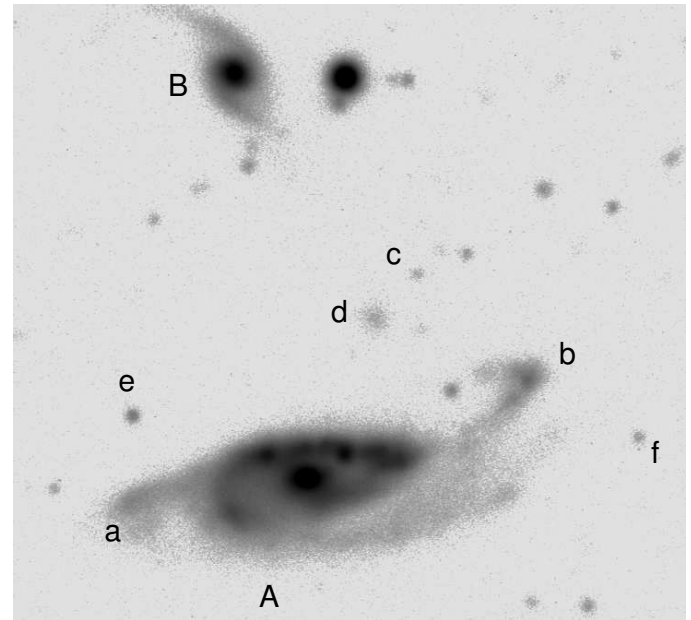

Figure 7: Identification chart of field 3 around AM 0547-244.

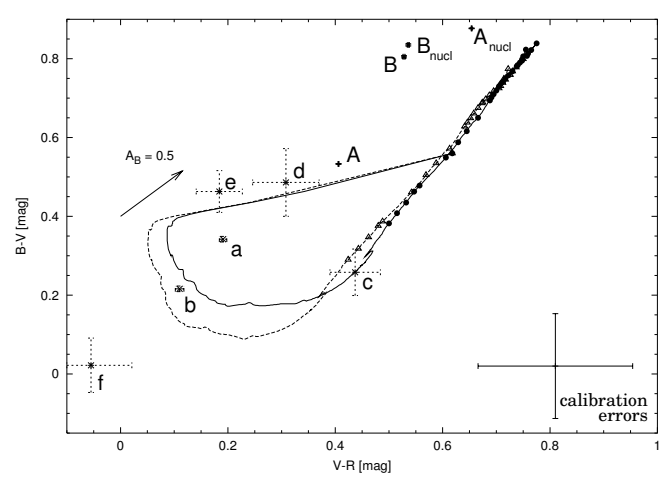

Figure 8: Plot of the objects in field 3 of AM 0547-244. The models used are $5\left(Z_{1}\right)$ (solid, $b=0.09$ ) and $6\left(Z_{1}\right)$ (dotted, $\left.b=0.18\right)$.

and all other unnamed visible extended objects in the field are found to be background galaxies from their colors.

Several large blue knots probably associated with star-forming regions can be seen in the tails. They are labeled "a" to "i". Fig. 6 shows that all knots are well described by the $Z_{3}$ model with a burst strength of the order of $10 \%$. They have similar burst ages of around 3 Myrs.

\subsection{AM 0547-244}

This system (Fig. 7) consists of a peculiar galaxy "A" $\left(V_{\mathrm{A}}=13164 \mathrm{~km} \mathrm{~s}^{-1}\right)$, from which three tidal tails emanate, and a companion "B", the redshift of which is unknown. The colors of the nuclei of "A" and "B" are very red (see Fig. 8). This could indicate an 'old' burst age or strong reddening due to large amounts of dust.

At the ends of the tails of galaxy "A" two large condensations are visible, denoted by "a" and "b". With absolute magnitudes of $M_{B_{\mathrm{a}}}=-16.94$ and $M_{B_{\mathrm{b}}}=-17.05$, "a" and "b" are good candidates for luminous TDGs. Around "A" four more knots with unknown redshift can be seen ("c" to "f"). All other objects have very red colors and are therefore most probably background objects.

Fig. 8 shows that the objects "a" to "d" agree well with the models with metallicity $Z_{1}$ and intermediate to high burst strengths while " $\mathrm{f}$ " is not matched by any model. The interpretation of " $\mathrm{a}$ " to " $\mathrm{f}$ " is severely limited by the large systematic errors of this field. $\mathrm{A} Z_{3}$ metallicity model can therefore not be excluded. 


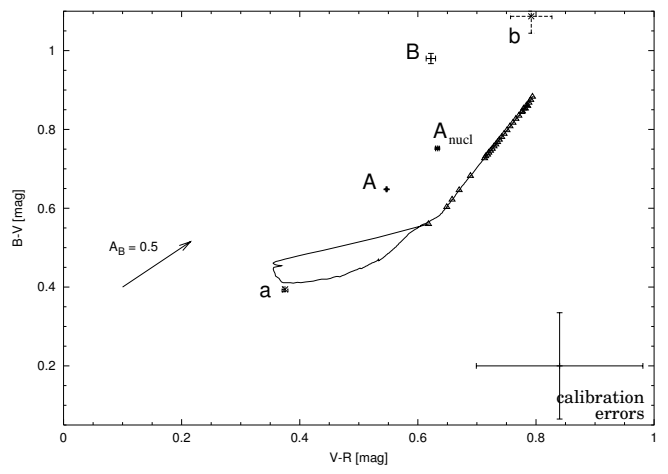

Figure 10: Plot of the objects in field 4 of AM 0607-444. The model

Figure 9: Identification chart of field 4 around AM 0607-444. used is $1\left(Z_{1}\right)$ (solid) $b=0.01$.

\section{4 АM 0607-444}

This system consists of a spiral galaxy ("A") which is severely disturbed in its outer parts (Fig. 9). The object labeled "B" has red colors and may be a background galaxy. Two objects are seen towards the northern tail. The very blue knot at its tip, "a", hosts a star-forming region. In contrast, " $b$ " is much redder, has elliptical contours in its center and may either be the remnant core of the interacting companion of "A" or a background galaxy superimposed on the tail. The colors of the TDG candidate "a" are best matched with a $Z_{1}$ model with a weak burst (Fig. 10), note, however, the large systematic errors of this field.

\subsection{AM 0642-645}

The peculiar system "A" (Fig. 11) exhibits two red nuclei ("a","b") and a bluer central knot, "c", and may well be a merger. A plume to the southeast is designated with " $\mathrm{d}$ " and nine other extended objects in the field of view that have colors in the range of our models have been labeled. Fig. 12 shows that all of these objects would be consistent with TDG models having weak to intermediate burst strengths with the exception of object " $f$ ", which has colors similar to an elliptical galaxy at 


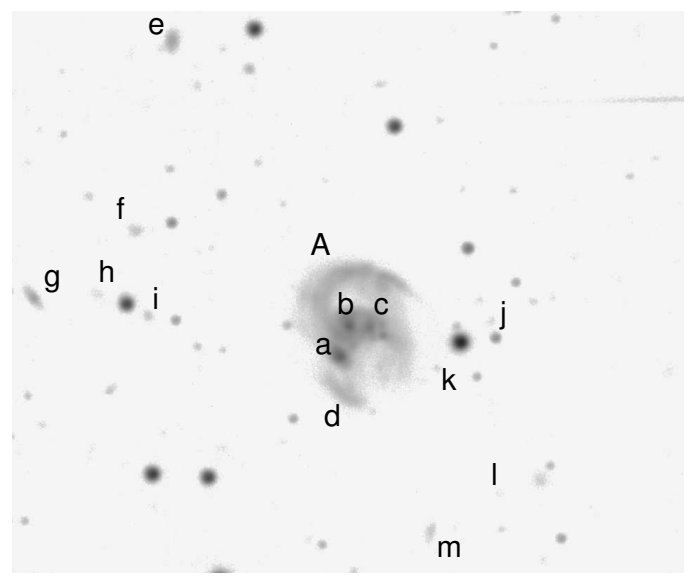

Figure 11: Identification chart of field 5 around AM 0642-645.

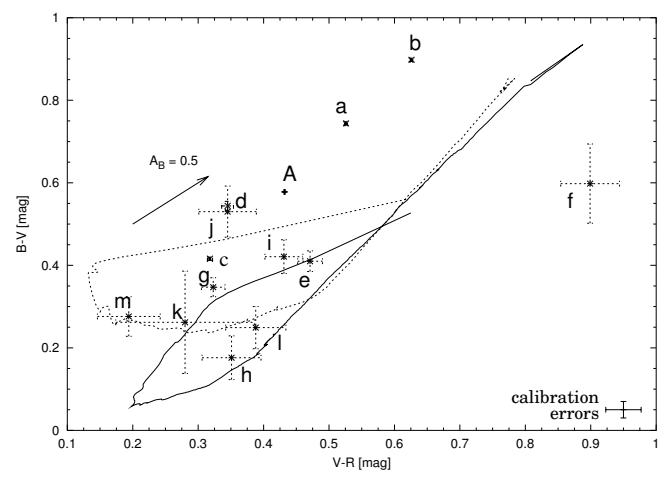

Figure 12: Plot of the objects in field 5 of AM 0642-645. The models used are $2\left(Z_{1}\right)$ (dotted, $b=0.05$ ) and $5\left(Z_{3}\right)$ (solid, $b=0.09$ ).

$z \approx 1.3$ (Möller et al. in prep.). However, without a spectroscopic redshift and in the absence of tidal structures linking them with the parent galaxy, the tidal origin of the objects surrounding " $A$ " is very speculative.

\subsection{AM 0748-665}

This interacting system is composed of two galaxies (" $\mathrm{A}$ " and " $\mathrm{B}$ ") which are connected by a long narrow bridge (Fig. 13). Their morphology indicates that the progenitors of "A" and "B" may have been a spiral and an elliptical, respectively. Several condensations labeled "a" to "e" can be seen in the associated tidal features.

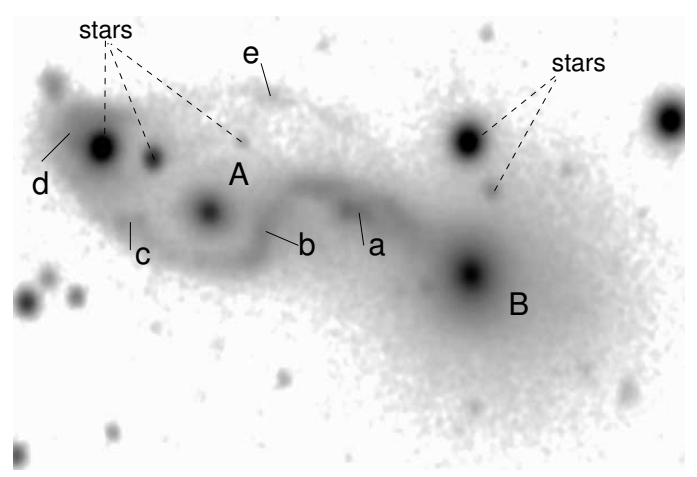

Figure 13: Identification chart of field 6 around AM 0748-665.

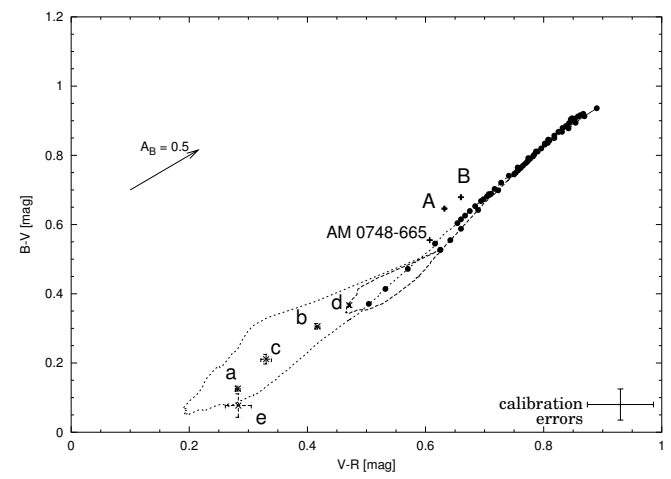

Figure 14: Plot of the objects in field 6 of AM 0748-665. The models used are $1\left(Z_{3}\right)$ (dashed) and $3\left(Z_{3}\right)$ (dotted) with $b=0.01,0.10$. 


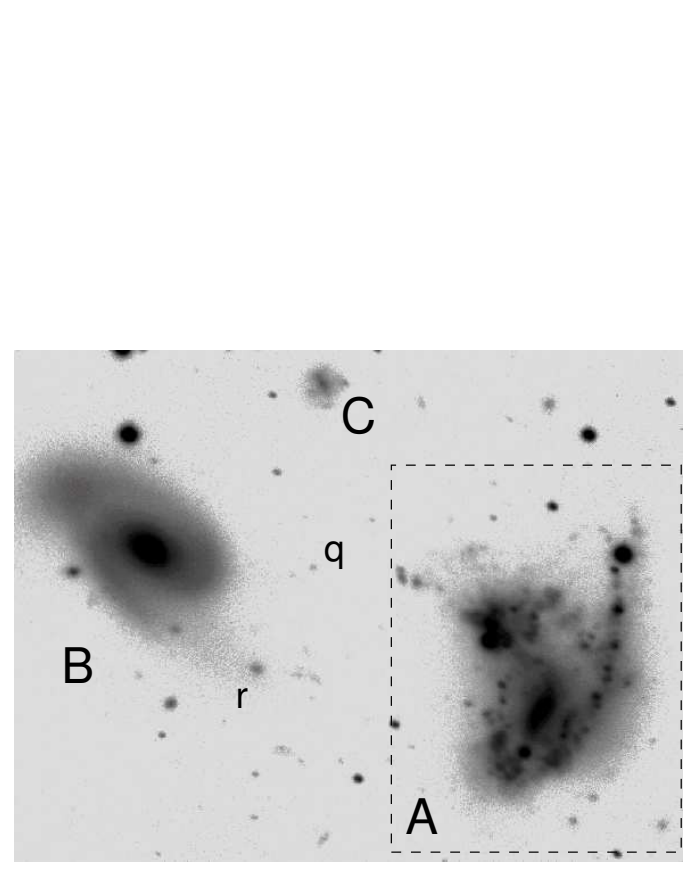

Figure 15: Identification chart of field 7 around AM 1054-325.

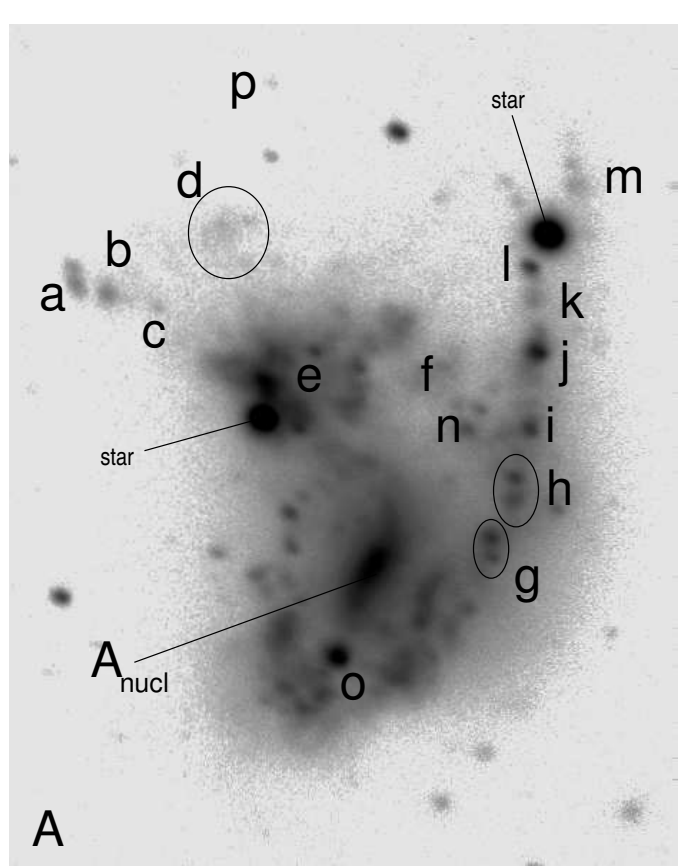

Figure 16: Identification chart of AM 1054-325A.

The brightest condensation " $\mathrm{d}$ " is blended with a bright star. The latter has been subtracted with a scaled PSF before carrying out the photometry. Fig. 14 shows that all data points agree with $Z_{3}$ models that provide burst strengths up to 0.10 .

\subsection{AM 1054-325}

This presumably interacting system (Fig. 15) is composed of an irregular H IIgalaxy, "A", at a redshift of $V_{\mathrm{A}}=3795 \mathrm{~km} \mathrm{~s}^{-1}$ (Sekiguchi \& Wolstencroft 1993) and a spiral-like object "B", which has however colors that are redder and more homogeneous than typical spiral galaxies. Its redshift is not known. Object " $\mathrm{C}$ " is a LSB galaxy at an unknown redshift.

The enlarged image of "A" (Fig. 16) indicates that this galaxy seems to have two nuclei, one marked as "Anucl", the other as " $\mathrm{e}$ " (blended by a foreground star). Like in AM 0529-565 A the two nuclei have different colors. The former is redder ( $B-$ $V=0.52)$ while the latter has the blue colors of a strong starburst $(B-V=0.21)$. Peña et al. (1991) derived for "A" an oxygen abundance of $12+\log (O / H)=8.14$ equivalent to $Z=0.003$. We therefore use our $Z_{1}$ model for this galaxy.

In the western tail of "A" several large star-forming regions are visible (" $\mathrm{g}$ " to " $\mathrm{m}$ ") with luminosities of fainter dwarf galaxies. A few other features of special interest are marked as "a" to "d" and "f". Fig. 17 shows that their colors are well matched 


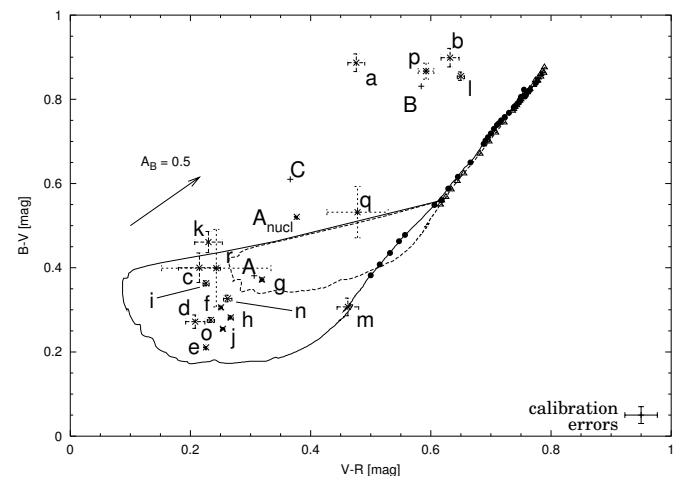

Figure 17: Plot of the objects in field 7 of AM 1054-325. The models used are $4\left(Z_{1}\right)$ (dashed, $\left.b=0.02\right)$ and $5\left(Z_{1}\right)$ (solid, $b=0.09$ ).

with $Z_{1}$ models with $0.02<b<0.09$. However "a", "b" and "p" that have discrepant reddish colors are likely background objects. Note that the outlier "l" has a very uncertain photometry due to the vicinity of a bright saturated star.

\subsection{AM 1208-273}

This system, also known as MCG-5-29-28, has three apparent members (Fig. 18). The main galaxy, "A", is a disturbed Sc spiral with a small bar visible at the center $\left(V_{\mathrm{A}}=14780 \mathrm{~km} \mathrm{~s}^{-1}\right)$. "B" is a foreground object with an active starburst $\left(V_{\mathrm{B}}=\right.$ $12433 \mathrm{~km} \mathrm{~s}^{-1}$, Donzelli \& Pastoriza 1997). The redshift of the disk galaxy " $\mathrm{C}$ " is not known.

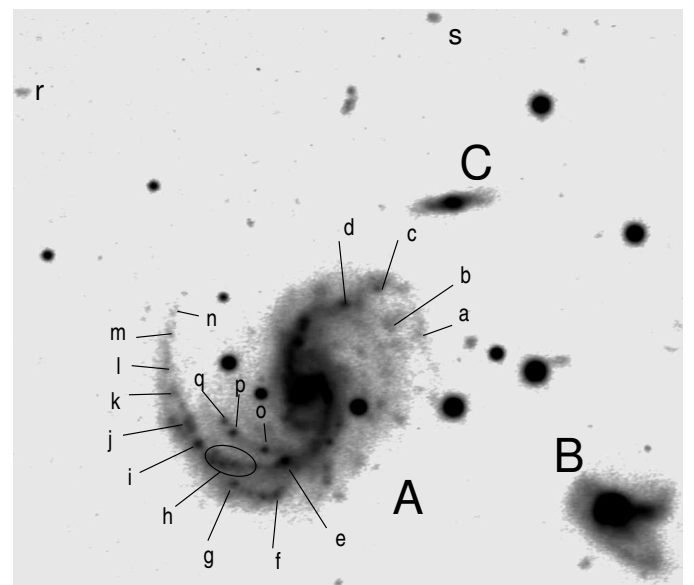

Figure 18: Identification chart of field 8 around AM 1208-273.

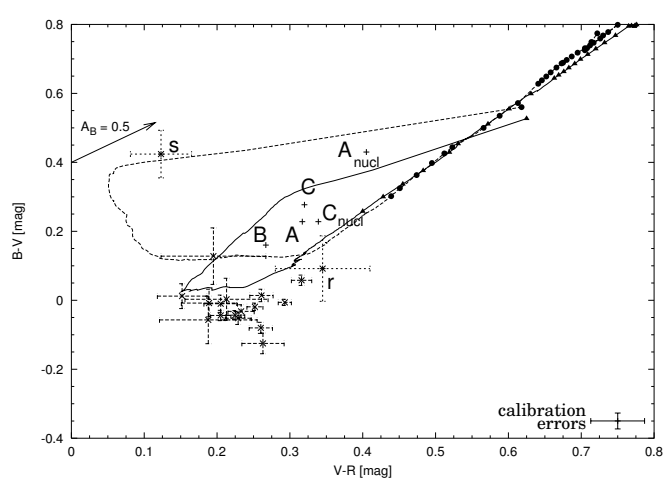

Figure 19: Plot of the objects in field 8 of AM 1208-273. The models used are $6\left(Z_{1}\right)$ (dashed) and $6\left(Z_{3}\right)$ (solid) both with $b=0.18$. 


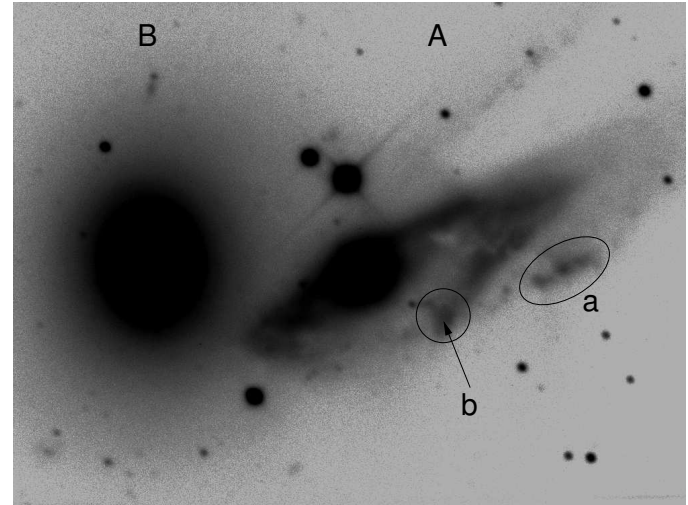

Figure 20: Identification chart of field 9 around AM 1325-292.

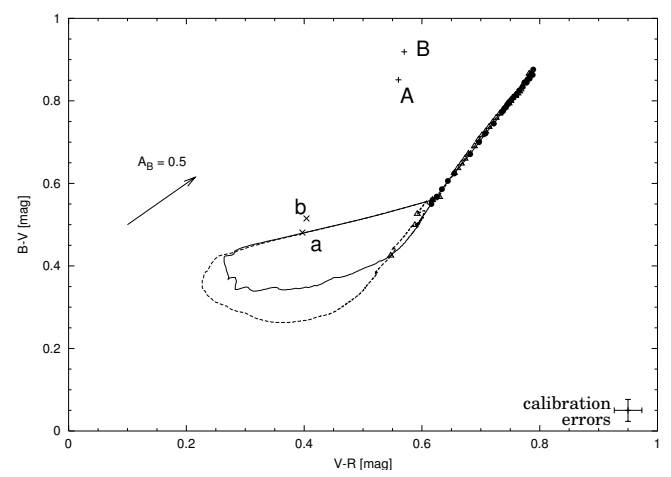

Figure 21: Plot of the objects in field 9 of AM 1325-292. The models used are $4\left(Z_{1}\right)$ (solid, $b=0.02$ ) and $7\left(Z_{1}\right)$ (dashed, $\left.b=0.04\right)$.

Along the extended spiral arms of " $A$ " several knots are visible, marked as "a" to "q". Two detached diffuse objects " $r$ " and "s" are visible northeast and north of the main galaxy, but their association with the other galaxies is uncertain. Fig. 19 shows that all knots in the arms are matched by a $Z_{3}$ model with a high burst strength. They have roughly the same burst age.

\subsection{AM 1325-292}

AM 1325-292 (Fig. 20) is a close interacting system between a spiral, NGC 5152 ("A") and an elliptical, NGC 5153 ("B"). Their redshifts are $V_{\mathrm{A}}=4598 \mathrm{~km} \mathrm{~s}^{-1}$ and $V_{\mathrm{B}}=4431 \mathrm{~km} \mathrm{~s}^{-1}$, respectively.

The spiral has three visible spiral arms/tidal tails, a long one to the north and two apparently shorter ones to the southwest, at the end of which two bigger knots of dwarf galaxy size are seen ("a" and "b"). These knots have the bluest colors of the entire system and do well agree with $Z_{1}$ models and small or intermediate burst strength shown in Fig. 21. The small photometric errors preclude $Z_{3}$ models although due to the nature of the parent galaxy a higher metallicity would have been expected.

\subsection{AM 1353-272}

Four galaxies are seen in the field of AM 1353-272 (Fig. 22). The two brightest are at the same redshift. "A" is an integral-sign spiral $\left(V_{\mathrm{A}}=12145 \mathrm{~km} \mathrm{~s}^{-1}\right)$ interacting with a disturbed disk galaxy " $\mathrm{B}$ " $\left(V_{\mathrm{B}}=11791 \mathrm{~km} \mathrm{~s}^{-1}\right)$. "C" is most probably a background elliptical, as judged from its red colors. " $D$ ", to the southeast, is a LSB 


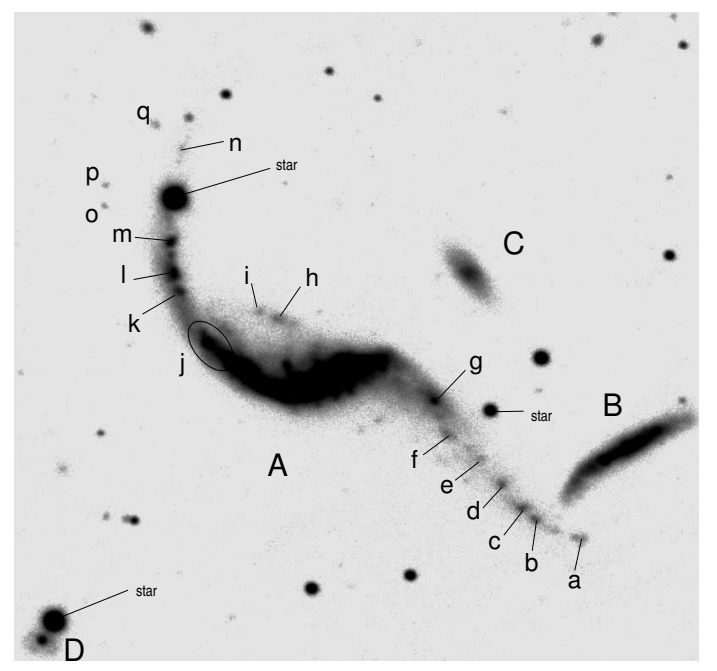

Figure 22: Identification chart of field 10 around AM 1353-272.

galaxy. Its color is highly uncertain due to the contamination by a bright foreground star.

The long tidal tails of "A" host a number of blue knots ("a" to " $n$ "). Three detached small knots ("o" to "q") are visible east of the northeast tail. Given their colors, these knots could be physically linked with " $A$ " but without redshift information available this remains speculative. The knots have absolute magnitudes in the range of $-12.1<M_{B}<-15.7$ mag. Their colors do agree with the $Z_{3}$ models and in-

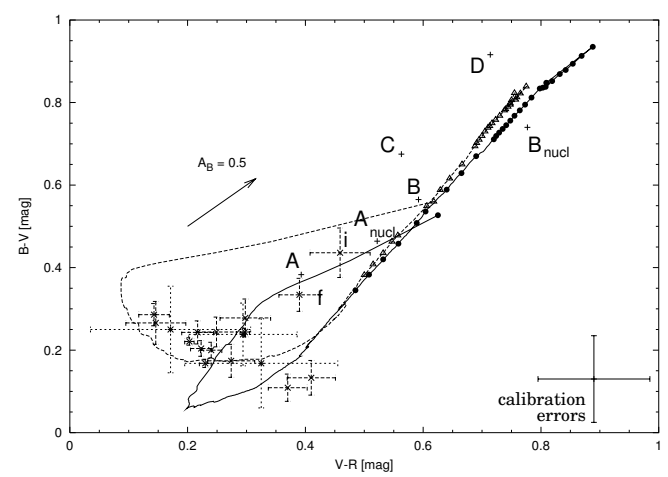

Figure 23: Plot of the objects in field 10 of AM 1353-272. The models used are $4\left(Z_{1}\right)$ (dashed, $b=$ $0.02)$ and $6\left(Z_{3}\right)$ (solid, $b=0.18$ ).

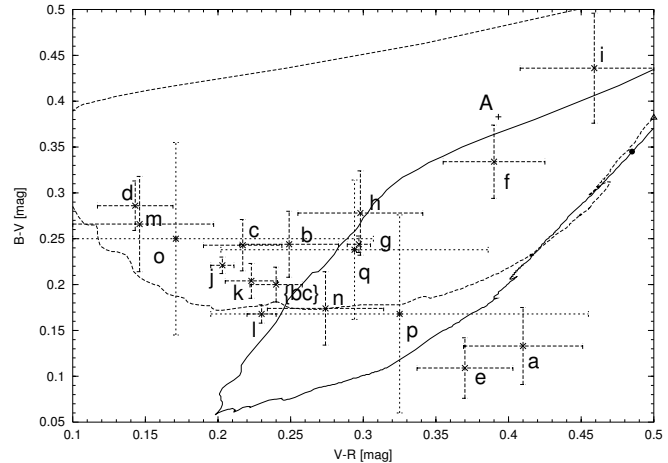

Figure 24: Close-up of Fig. 23. The knots are now labeled. 
termediate strength burst. Condensations " $\mathrm{d}$ " and " $\mathrm{m}$ " have however deviant colors that seem more consistent with $Z_{1}$ models.

\section{Discussion}

\subsection{Background contamination}

In each field a number of faint extended objects are visible (see e.g. Figs. 5 and 7) that have red colors. They lie outside the color range of our models that are valid for $z=0$ only. Most of them should hence be background objects. This was tested using the chemically consistent evolutionary synthesis models of Möller et al. (1998) and Möller et al. (in prep.), which include cosmological parameters and give colors of all galaxy types as a function of $z$. A rough photometric redshift could be determined from the $B, V, R$ measurements. The few faint objects with colors that strongly deviate from our TDG models and that are inconsistent with those of distant galaxies could be red dwarf galaxies pre-existing the collisions. They have been excluded from our analysis. One SUSI field with an area of 1.3 $10^{-3} \square^{\circ}$ contains on average $7.0 \pm 0.7$ and $13.0 \pm 4.0$ objects with deviant colors in the respective magnitude ranges $B=22 \ldots 23$ and $B=23 \ldots 24$. After correction for the occlusion of background objects due to the interacting system (typically $2 \cdot 10^{-4} \square^{\circ}$ ), these values are compatible with those of Metcalfe et al. (1991). They imaged 12 fields with an area of $6.8 \cdot 10^{-3} \square^{\circ}$ and counted $5000 \pm 800$ and $15000 \pm$ 1000 galaxies, respectively, for the magnitude ranges in question. For our field of view, the numbers would be $6.8 \pm 1.1$ and $20.2 \pm 1.3$. Therefore we do not see an overdensity of background galaxies in our fields contrary to what Deeg et al. (1998) found in their sample of interacting systems. Their field of view is much larger (about $3.3 \cdot 10^{-3} \square^{\circ}$ ) than ours and they have better statistics. But they mostly imaged interacting systems with lower $z$ which hence occlude a larger portion of the image. Neither did they use color information to preselect possible nearby objects. Moreover, they mainly found an overdensity in the brightness range $R=18 \ldots 19.5$ mag, where we do not have enough background objects for statistical analysis.

\subsection{Properties of the TDG candidates}

As seen before, several knots turn out to be background objects as judged from their colors. One should hence be cautious when physically linking faint objects with apparently nearby large galaxies. This is especially true for the detached objects lying far away from the tidal tails of the parent galaxies. In the absence of a redshift measurement, the color information and its comparison with the model predictions is useful to disentangle between possible objects in or close to tidal fea- 


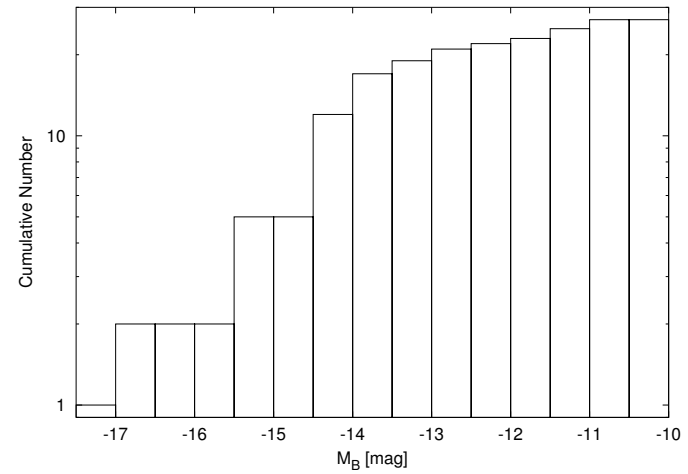

Figure 25: Luminosity function of the tidal objects.

tures from background galaxies. Using this technique, we have compiled a catalog of candidates for TDGs or their progenitors, i.e. knots associated with or close to tidal features that - within the observational errors - agree with one or more of our evolutionary synthesis models. They are listed in Table 4.

Column 1 shows the designation of the candidate. Column 2 gives, when its distance is known, the absolute blue magnitude of the TDG candidate. Columns 3 and 4 resp. give its $B-V$ color and the difference in $B-V$ between the knot and the surrounding tail (values in parentheses indicate detached knots). The last three columns list the results from the comparison with the models. Column 5 gives the ID of the best matching model(s), column 6 the most probable burst age(s), and column 7 a range of possible burst strengths. Note that the best-fit models shown here for every individual TDG candidate are not necessarily those shown in the plots in Sect. 4, where models were chosen to represent a maximum number of data points.

\section{Number and luminosity}

In our sample of ten, 8 interacting systems contain promising TDG candidates. On average 3.6 were identified per system. The luminosities of the candidates for which the distance of their parent galaxies is known range from faint $\left(M_{B}=-10.4 \mathrm{mag}\right)$ to brighter dwarf galaxies $\left(M_{B}=-17.1 \mathrm{mag}\right)$ with a mean of $\left\langle M_{B}\right\rangle=-13.6 \mathrm{mag}$. It should be noted that even though we have some faint TDG candidates in our sample, they are still more luminous than normal $\mathrm{H}_{\mathrm{II}}$ regions in spiral galaxies by about 4 mag (Bresolin \& Kennicutt 1997). The luminosity function of the TDG candidates is shown in Fig. 25. 
Table 4: TDG candidates

\begin{tabular}{|c|c|c|c|c|c|c|}
\hline Designation & $\begin{array}{c}M_{B} \\
{[\mathrm{mag}]}\end{array}$ & $\begin{array}{l}B-V \\
{[\mathrm{mag}]}\end{array}$ & $\begin{array}{c}\Delta(B-V) \\
\text { (tail-TDG) }\end{array}$ & $\begin{array}{c}\text { Best fit } \\
\text { Model ID }\end{array}$ & $\begin{array}{c}\text { burst age } \\
\text { [Myr] }\end{array}$ & $\begin{array}{c}\text { burst strength } \\
\qquad b\end{array}$ \\
\hline AM 0529-565a & -11.48 & 0.37 & $(0.03)$ & $4\left(Z_{1}\right)$ & 4 & 0.02 \\
\hline AM 0529-565b & -10.65 & 0.52 & $(-0.12)$ & $6\left(Z_{1}\right)$ & $<1$ & $0.01 . .0 .18$ \\
\hline AM 0529-565g & -11.64 & 0.41 & 0.01 & $4\left(Z_{1}\right)$ & 3 & $0.02 . .0 .05$ \\
\hline AM 0529-565i & -12.57 & 0.42 & 0.15 & $6\left(Z_{1}\right)$ & $<1$ & $0.05 . .0 .18$ \\
\hline AM 0537-292a & & 0.31 & 0.15 & $6\left(Z_{3}\right)$ & 1 & $0.04 . .0 .18$ \\
\hline AM 0537-292d & & 0.18 & 0.28 & $5\left(Z_{3}\right)$ & $10 / 3$ & $0.05 . .0 .18$ \\
\hline AM 0537-292e & & 0.21 & 0.20 & $5\left(Z_{1}\right)$ & $8 / 3$ & $0.09 . .0 .18$ \\
\hline AM 0537-292f & & 0.28 & 0.11 & $5\left(Z_{3}\right)$ & $\approx 1$ & $0.05 . .0 .10$ \\
\hline AM 0537-292g & & 0.21 & 0.16 & $3\left(Z_{3}\right)$ & $10 / 3$ & $0.09 . .0 .18$ \\
\hline AM 0547-244a & -16.94 & 0.34 & 0.07 & $9\left(Z_{1}\right)$ & 4 & $0.04 . .0 .05$ \\
\hline AM 0547-244b & -17.05 & 0.22 & 0.12 & $3\left(Z_{1}\right)$ & $<1 . .4$ & $0.09 . .0 .18$ \\
\hline AM 0547-244d & -14.46 & 0.49 & $(-0.15)$ & $6\left(Z_{1}\right)$ & $<1$ & $0.02 . .0 .18$ \\
\hline AM 0607-444a & & 0.39 & 0.34 & $1\left(Z_{1}\right)$ & $<1 / 6$ & $0.01 . .0 .18$ \\
\hline AM 0748-665a & & 0.13 & 0.32 & $2\left(Z_{3}\right)$ & $3 / 10$ & 0.05 \\
\hline AM 0748-665d & & 0.21 & $0.11^{1}$ & $1\left(Z_{3}\right)$ & 20 & 0.01 \\
\hline AM 0748-665e & & 0.08 & 0.15 & $3\left(Z_{3}\right)$ & 10 & $0.05 . .0 .18$ \\
\hline AM 1054-325g & -14.29 & 0.37 & 0.08 & $4\left(Z_{1}\right)$ & 5 & 0.02 \\
\hline AM $1054-325 h$ & -13.73 & 0.28 & 0.11 & $7\left(Z_{1}\right)$ & 6 & 0.04 \\
\hline AM 1054-325i & -13.25 & 0.36 & -0.02 & $7\left(Z_{1}\right)$ & 4 & 0.04 \\
\hline AM $1054-325 j$ & -14.36 & 0.26 & 0.12 & $2\left(Z_{1}\right)$ & 6 & 0.05 \\
\hline AM $1054-325 k$ & -10.40 & 0.46 & -0.14 & $6\left(Z_{1}\right)$ & $<1$ & $0.05 . .0 .18$ \\
\hline AM 1054-325m & -11.40 & 0.31 & $0.08^{1}$ & $5\left(Z_{1}\right)$ & 70 & $0.05 . .0 .18$ \\
\hline AM 1325-292a & -15.30 & 0.48 & 0.15 & $5\left(Z_{1}\right)$ & $<1$ & $0.01 . .0 .18$ \\
\hline AM $1325-292 b$ & -15.46 & 0.52 & 0.18 & $6\left(Z_{1}\right)$ & $<1$ & $0.01 . .0 .18$ \\
\hline AM 1353-272a & -13.68 & 0.13 & $(0.19)$ & $7\left(Z_{3}\right)$ & 40 & $0.04 . .0 .10$ \\
\hline AM 1353-272b & -13.10 & 0.24 & 0.07 & $2\left(Z_{3}\right)$ & $9 / 3$ & $0.04 . .0 .18$ \\
\hline AM $1353-272 c$ & -13.58 & 0.24 & 0.02 & $2\left(Z_{3}\right)$ & 8 & 0.05 \\
\hline AM $1353-272 d$ & -13.98 & 0.29 & 0.10 & $2\left(Z_{1}\right)$ & 4 & 0.05 \\
\hline AM 1353-272e & -14.03 & 0.11 & 0.27 & $5\left(Z_{3}\right)$ & 30 & $0.05 . .0 .10$ \\
\hline AM 1353-272f & -12.84 & 0.33 & 0.05 & $4\left(Z_{3}\right)$ & $<1 / 20$ & $0.02 . .0 .18$ \\
\hline AM $1353-272 h$ & -14.01 & 0.34 & 0.21 & $6\left(Z_{3}\right)$ & $2 / 10$ & $0.04 . .0 .18$ \\
\hline AM 1353-272i & -12.13 & 0.44 & 0.04 & $1\left(Z_{3}\right)$ & $<1 / 40$ & $0.01 . .0 .18$ \\
\hline AM $1353-272 k$ & -13.99 & 0.20 & 0.11 & $5\left(Z_{1}\right) / 6\left(Z_{3}\right)$ & $8 / 3$ & $0.05 . .0 .18$ \\
\hline AM 1353-2721 & -15.25 & 0.17 & 0.12 & $5\left(Z_{1}\right) / 6\left(Z_{3}\right)$ & $9 / 3$ & $0.09 . .0 .18$ \\
\hline AM 1353-272m & -14.49 & 0.14 & 0.03 & $2\left(Z_{1}\right)$ & 4 & $0.05 . .0 .10$ \\
\hline AM $1353-272 n$ & -14.31 & 0.17 & 0.13 & $2\left(Z_{3}\right)$ & $10 / 3$ & $0.05 . .0 .10$ \\
\hline
\end{tabular}

${ }^{1}$ Contamination by a nearby bright star. 


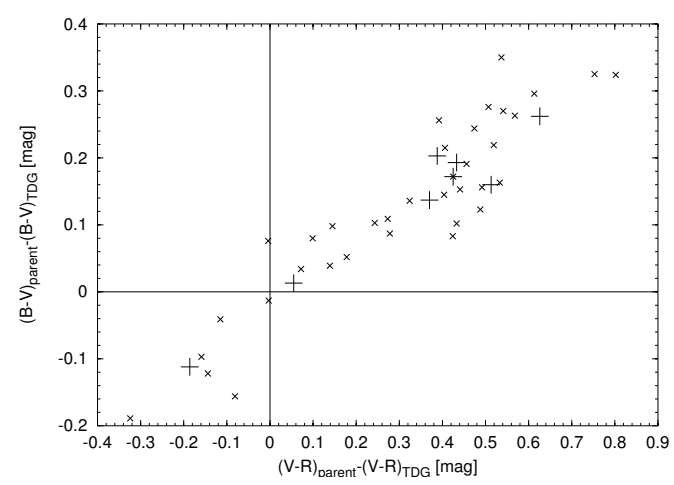

Figure 26: Two-color-diagram of the differential colors of parent galaxies and TDG candidates. The small crosses mark the TDG candidates selected in Table 4. The big crosses mark the mean value of all TDG candidates in one given system.

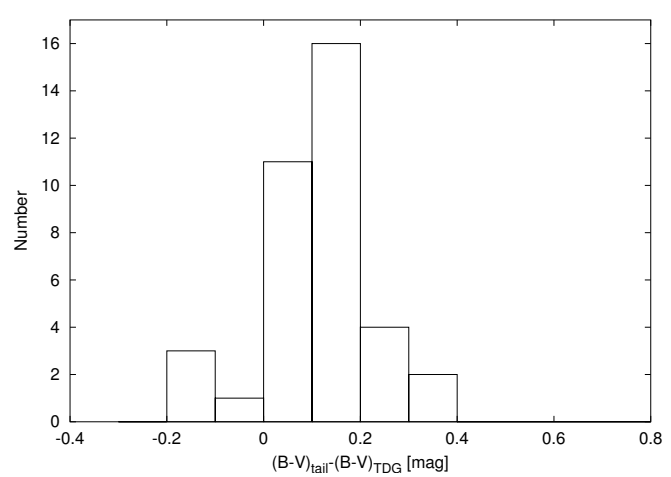

Figure 27: Histograms of the differential colors of tidal features and the TDG candidates in $B-V$.

\section{Colors and star formation}

In Fig. 26 we compare the colors of these knots with the overall colors of their parent galaxy. Note that the measurement of the parent galaxy includes the light of the knots, but this does not have a strong effect as the knots only give a neglegible contribution (up to $1 \%$ of the flux) to the brightness of the galaxy.

On average the TDG candidates have bluer colors than their parent galaxies, which could be due to a number of effects:

- Fading effect: only the bluest clumps are visible. As we have selected the candidates from their surface brightness in the $V$-band images and all candidates have a young burst age, it is possible that after a longer burst the objects become redder and fade in luminosity and are no longer visible as surface brightness peaks in $V$.

- High relative gas content: the knots may experience stronger starbursts than the parent galaxies. A prerequisite for this could be a high gas content in the tidal features as observed in other systems (e.g. Duc \& Mirabel 1998).

- Time difference: The burst in the parent galaxies may take place before the star formation in the tidal features starts. The color of the parent galaxy would then be dominated by the color of the red central part, especially after a strong nuclear starburst has faded. 
- Smaller dust content: If the knots have a smaller dust extinction than the parent galaxies, their colors would appear redder.

- The colors of the parent galaxies are largely dominated by the colors of their nuclei which are usually redder than the disk from which a TDG may form.

In order to tackle the last bias, we have compared the colors of the knots with the color of their immediate surroundings, i.e. in the tails. For knots already detached from the visible tail, the nearest part of the tail was taken for the measurement. The results are shown in Table 4, column 4 and in Fig. 27. Nearly all knots have a $B-V$ color bluer than their surrounding tidal features. We therefore conclude that the knots are actively forming stars while their surrounding material is more quiescient. As already noticed by Schombert et al. (1990), the tidal features and especially the surface brightness peaks which we identify as TDG candidates often show the bluest $B-V$ colors of the entire interacting system.

Some examples of interacting galaxies show star formation in the tails but already have postburst characteristics in the central part of the merger (e.g. Hibbard et al. 1994). One could therefore conclude that star formation propagates from the central part to the outer regions along the tails and look for such an effect in interacting systems with several condensations along the tails. We do not find, however, any clear trends in the properties of the knots along the tails in the two systems which have longer tails (AM 1054-325A and AM 1353-272A) neither in luminosity or color, nor in burst strength or burst age.

\section{Burst properties}

The model bursts developed in this study that best fit the TDG candidates require intermediate to strong burst strength $(0.01<b<0.18)$. Taking into account possible internal extinction would favor even stronger bursts. These burst strengths therefore seem to be higher than for BCDGs, which have typical strengths of $b \approx 0.01$ or less (Krüger 1992). The burst strengths of our TDG candidates also indicate that the mass is still governed by the old stars $(b<0.20)$, but the luminosities in $B$ and $R$ are already dominated by the young component (see Table 2 ).

The mean burst age of the tidal objects is about 8 Myrs. Their dynamical timescale may be determined assuming a typical distance of the knots from the nucleus of the parent galaxy of $15 \mathrm{kpc}$ - about half of the length of the tails of AM 1353-272 - and a typical rotation velocity in a spiral galaxy of $150 \mathrm{~km} \mathrm{~s}^{-1}$. With these values, disk material is expelled to the present positions of the knots in about 100 Myrs. Only very few objects in our sample show comparable high burst ages. This suggests that the star formation episode in the TDG candidates has started in situ in the tidally expelled material. 


\subsection{Nature of the TDG candidates}

The condensations in the tidal tails which we call "Tidal Dwarf Galaxy candidates" show properties in the range expected for TDGs. We call them candidates, because they still lack spectroscopic confirmation and especially the proof that they are bound objects.

The TDG candidates identified in the systems presented here are on average less luminous but more numerous than the TDGs found in other interacting systems studied up to now. These other TDGs have an absolute blue magnitude of $-15.5 \mathrm{mag}$ on average, part of them have been found to be kinematically decoupled from their tails (Duc et al. 1997, Duc \& Mirabel 1998), and one or two of them are produced per collision. Moreover, the TDGs known to be gravitationally bound are found at the tip of the optical tails whereas our TDG candidates are distributed all along the tidal features without any trend in luminosity in agreement with one of the models discussed above. One reason for these differences may lie in the morphologies of the parent galaxies. TDGs have so far essentially been observed in merging systems involving massive spirals. Some of the interacting systems presented here are composed of late type galaxies, some of which have luminosities low enough to be classified as dwarf galaxies (e.g. AM 0529-565). It thus does not seem surprising that they form less massive condensations.

\section{Formation of bound objects}

Numerical models of galaxy collisions show the presence of bound objects in tidal debris that have luminosities and distributions quite similar to the TDG candidates studied in our sample (e.g. Barnes \& Hernquist 1992). The physical process for their formation is still largely unknown. They might either form from growing instabilities in the stellar component of tidal debris that may or may not attract surrounding gas (Barnes \& Hernquist 1992), or alternatively from expelled gas that collapses and forms stars as proposed by Elmegreen et al. (1993). The blue colors of our TDG candidates favor the second scenario. Indeed we did not find any evidence for the presence for pure stellar condensations in the tidal features of our interacting systems. Our models show that recent star formation with a minimum strength of $b \approx 0.01$, i.e. forming $1 \%$ of young stars is necessary to account for the colors of our TDG candidates. In most cases, however, a much stronger burst is required. Note however, that we cannot distinguish between a stellar condensation into which gas falls later to cause the starburst and an initially gaseous condensation that forms stars on an underlying population of old stars. 


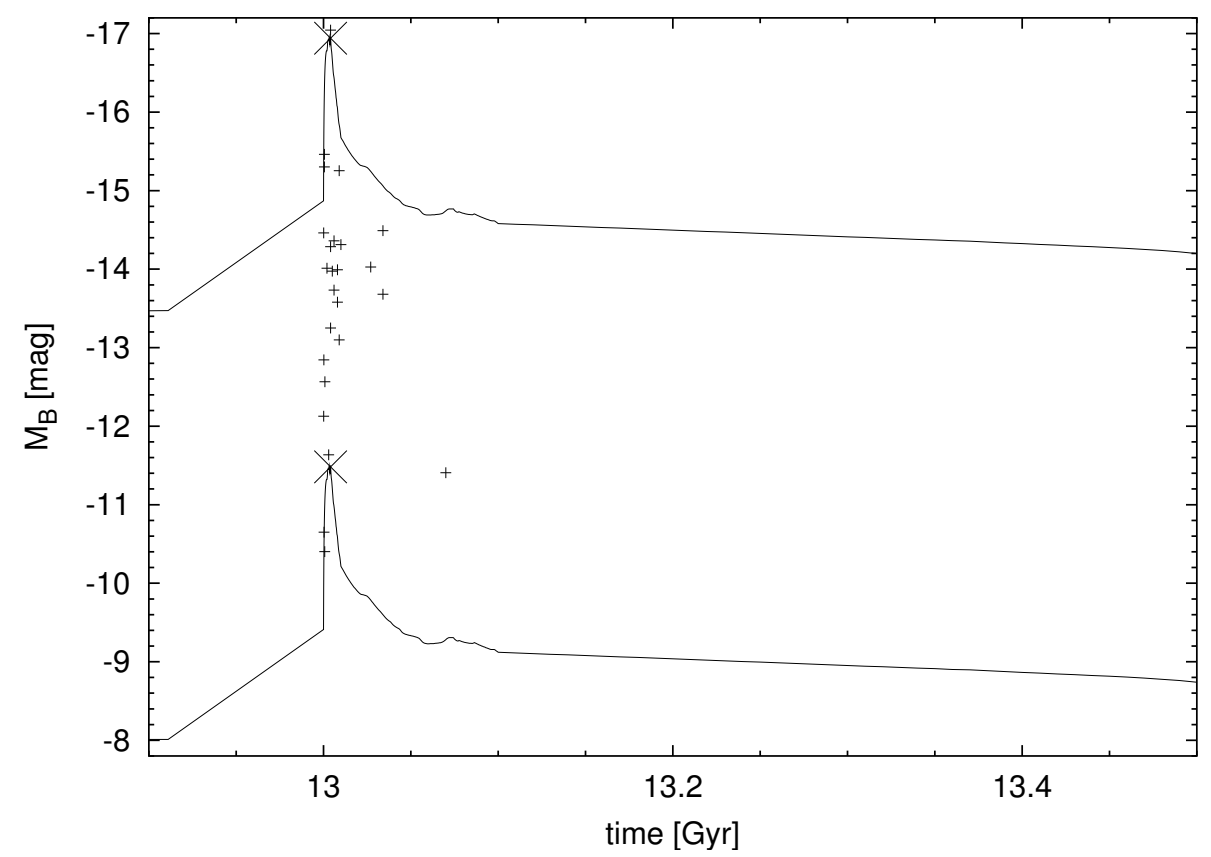

Figure 28: Luminosity evolution of the selected TDG candidates which are displayed as crosses according to their luminosity and burst age. The models shown are $06\left(Z_{1}\right)$ with $b=0.18$ shifted in $M_{B}$ to fit the two candidates represented by the larger crosses. See text about discussion of these two examples.

\section{Future evolution}

The spectrophotometric models are also used to extrapolate the evolution of the knots, i.e. to predict how luminosities and colors will change with time. The latter is already visible on the two-color diagrams in Sect. 4, where the evolution is marked in steps of $100 \mathrm{Myr}$ along the model curves. Typically one Gyr after the burst, the optical colors of the condensations will become indistinguishable from the pre-burst colors unless further star formation episodes take place. The luminosity evolution is shown in Fig. 28, where models for one of the most luminous TDG candidates, AM 0547-244a, and one of the least luminous candidates, AM 0529$565 \mathrm{a}$, are displayed. Without secondary bursts, the $B$-luminosity will decrease by about 2.5 mag within $200 \mathrm{Myr}$ from the present age. AM 0529-565a, with a present day absolute magnitude of $M_{B}=-11.48$, will fade to $M_{B} \approx-8.0$ mag within 2 Gyrs. It will then have a luminosity similar to those of the faintest known galaxies of the local group (Mateo 1998). It would become invisible in our images at the distance of AM0529-565. On a similar timescale, the more luminous TDG candidate AM 0547-244a $\left(M_{B}=-16.94\right)$ will fade to $M_{B} \approx-13.4$ mag. It would be 
among the brighter dwarfs in the local group and could still be observed at its actual distance $\left(m_{B} \approx 22.8 \mathrm{mag}\right)$.

The formation of objects as massive as TDGs in tidal debris is still puzzling. One may wonder whether the numerous knots that we observe here and that are produced in numerical simulations of galaxy collisions could be the progenitors of the more massive TDGs identified before. As the photometric models indicate a rapid fading of the TDGs after the burst, the evolution of a TDG progenitor into a TDG would require that star formation in these condensations is sustained for at least $100 \mathrm{Myrs}$, supposing that the progenitors initially have a large enough gas reservoir. TDG progenitors could grow that way and reach masses and luminosities of the well known TDGs. On the other hand, by that time, some of the less active TDG progenitors might have faded and those initially situated close to their parent galaxies might have fallen back, explaining why classical TDGs are found preferentially at the end of tidal tails. Observations of a larger sample of systems and more detailed simulations would be necessary to confirm the link between our TDG candidates and more luminous TDGs.

Another possibility is that the TDG candidates in this paper do not evolve into larger TDGs. Instead some of our TDG candidates may not grow much and eventually form more fragile TDGs of lower luminosity. Even if fading after the burst is taken into account, our faintest TDG candidates still have luminosities comparable to those of faint local group dwarf galaxies. The question if they will be able to survive as bound and dynamically independent objects can only be answered by observations of the kinematics in combination with detailed dynamical models.

To be able to do more than speculate about the dynamical fate of the TDG candidates, kinematical data of the system and the mass of the gaseous component of each knot is required. To further restrict the mass of the underlying old component NIR observations will be needed.

\section{Conclusions}

We have presented imaging data of 10 southern interacting systems from the Arp $\&$ Madore Catalog selected for their resemblance to disturbed distant galaxies. Our high quality images reveal a heterogeneous set of morphologies from loosely interacting dwarf galaxies to disk-disk mergers. Through detailed photometry based on polygonal apertures in the three optical bands $B, V, R$ we were able to measure the colors of about one hundred condensations in or near the tidal features of the interacting galaxies.

We have computed a grid of evolutionary synthesis models simulating starbursts of various strengths in an underlying stellar population of mixed ages typical of spiral galaxies. Such models reproduce well the situation of TDGs and their progenitors 
that are composed of an old population pulled out from the parent galaxy and young stars formed in situ. Our models take into account the emission lines and the continuum from the photoionized gas with appropriate metallicities. Comparing our photometric data with these models, we selected 36 blue condensations as possible TDGs. Follow-up spectroscopy is required in some cases to assess their physical association with the interacting system.

We discussed the overall properties of the 36 TDG candidates, noting that they have luminosities brighter than $\mathrm{H}$ II regions in spiral galaxies and more typical for that of dwarf galaxies. Their color is much bluer than those of their parent galaxies and mostly bluer than the surrounding material in the tidal features. The contribution of young stars to their mass is at least $1 \%$ and may reach $20 \%$ or more for the most active condensations. In any case their $B$-band luminosity is dominated by this young population. The models also indicate that after the starburst when the young stars cease to be the dominating source the condensations may fade by up to 2.5 mag in $B$ within 200 Myrs after the burst. Although the TDG candidates seem to have young burst ages, we did not find any case of condensations solely made of old stars from the parent galaxies although the formation of such objects is also predicted in numerical simulations.

Finally the TDG candidates studied here have properties different from those of the more massive TDGs typically found at the tip of giant tidal tails of nearby interacting systems. We discuss whether our TDG candidates could be progenitors of TDGs or a class of less luminous objects.

We wish to thank the team at the NTT who made most of the observations under difficult conditions. We also thank our referee, E. Brinks, for careful reading of the manuscript and useful comments, which improved the paper. This research has made use of the NASA/IPAC Extragalactic Database (NED) which is operated by the Jet Propulsion Laboratory, California Institute of Technology, under contract with the National Aeronautics and Space Administration. PMW acknowledges partial support from DFG grant FR 916/6-1.

\section{References}

Appleton, P.N., Marston, A.P., 1997, AJ 113, 201

Arp, H.C., Madore, B.F., 1987, A Catalogue of Southern Peculiar Galaxies and Associations, Cambridge University Press, Cambridge

Barnes, J.E., Hernquist, L., 1992, Nature 360, 715

Bransford, M.A., Appleton, P.N., Marston, A.P., Charmandaris, V., 1998, AJ 116, 2757 
Bresolin, F., Kennicutt, Jr., R.C., 1997, AJ 113, 975

Burki, G., Rufener, F., Burnet, M., et al., 1995, The Messenger 80, 34

Burstein, D., Heiles, C., 1982, AJ 87, 1165

Charbonnel, C., Meynet, G., Maeder, A., Schaerer, D., 1996, A\&AS 115, 339

Charbonnel, C., Meynet, G., Maeder, A., Schaller, G., Schaerer, D., 1993, A\&AS 101, 415

Conselice, C., Bershady, M.A., Dickinson, M., et al., 1998, AAS 193, 7512

Deeg, H.J., Muñoz-Tuñón, C., Tenorio-Tagle, G., et al., 1998, A\&AS 129, 455

Donzelli, C.J., Pastoriza, M.G., 1997, ApJS 111, 181

Duc, P.-A., Brinks, E., Wink, J.E., Mirabel, I.F., 1997, A\&A 326, 537

Duc, P.-A., Mirabel, I.F., 1994, A\&A 289, 83

Duc, P.-A., Mirabel, I.F., 1997, The Messenger 89, 14

Duc, P.-A., Mirabel, I.F., 1998, A\&A 333, 813

Elmegreen, B.G., Kaufman, M., Thomasson, M., 1993, ApJ 412, 90

Ferguson, A.M.N., Gallagher, J.S., Wyse, R.F.G., 1998, AJ 116, 673

Fritze-von Alvensleben, U., Gerhard, O.E, 1994a, $A \& A$ 285, 751

Fritze-von Alvensleben, U., Gerhard, O.E, 1994b, A\&A 285, 775

Fritze-von Alvensleben, U., Möller, C.S., Duc, P.-A., 1998, in: Dwarf Galaxies and Cosmology (eds. T.X. Thuan, C. Balkowski, V. Cayatte, J. Trân Thanh Vân), Editions Frontières, Paris, 293

Hibbard, J.E., Guhathakurta, P., van Gorkom, J.H., Schweizer, F., 1994, AJ 107, 67

Hibbard, J.E., Mihos, J.C., 1995, AJ 110, 140

Izotov, Y.I., Thuan, T.X., Lipovetsky, V.A., 1994, ApJ 435, 647

Krüger, H., 1992, Blaue Kompakte Zwerggalaxien. Modellrechnungen zur metallizitätsabhängigen spektralen Entwicklung vom nahen Infrarot bis zum Röntgenbereich, $\mathrm{PhD}$ Thesis, Universität Göttingen 
Krüger, H., Fritze-von Alvensleben, U., Loose, H.-H., 1995, A\&A 303, 41

Landolt, A.U., 1992, AJ 104, 340

Leitherer, C., Heckman, T.M., 1995, ApJS 96, 9

Mateo, M.L., 1998, ARA\&A 36, 435

Metcalfe, N., Shanks, T., Fong, R., Jones, L.R., 1991, MNRAS 249, 498

Mirabel, I.F., Lutz, D., Maza, J., 1991, A\&A 243, 367

Möller, C., Fritze-von Alvensleben, U., Fricke, K.J., 1998, in: The Birth of Galaxies (eds. B. Guiderdoni, F.R. Bouchet, T.X. Thuan, J. Tran Thanh Van), Editions Frontières, in press

Peña, M., Ruiz, M.T., Maza, J, 1991, A\&A 251, 417

Savage, B.D., Mathis, J.S., 1979, ARA\&A 17, 73

Scalo, J.M., 1986, Fund. Cosmic Phys. 11, 1

Schaerer, D., Charbonnel, C., Meynet, G., Maeder, A., Schaller, G., 1993a, A\&AS 102, 339

Schaerer, D., de Koter, A., 1997, A\&A 322, 598

Schaerer, D., Meynet, G., Maeder, A., Schaller, G., 1993b, A\&AS 98, 523

Schaller, G., Schaerer, D., Meynet, G., Maeder, A., 1992, A\&AS 96, 269

Schombert, J.M., Wallin, J.F., Struck-Marcell, C., 1990, AJ 99, 497

Sekiguchi, K., Wolstencroft, R.D., 1993, MNRAS 263, 349

Stasińska, G., 1984, A\&AS 55, 15

van den Bergh, S., Abraham, R.G., Ellis, R.S., et al., 1996, AJ 112, 359

Zaritsky, D., Kennicutt, Jr., R.C., Huchra, J.P., 1994, ApJ 420, 87 
Paper I. 


\section{Paper II}

\section{Results from Optical Spectroscopy}

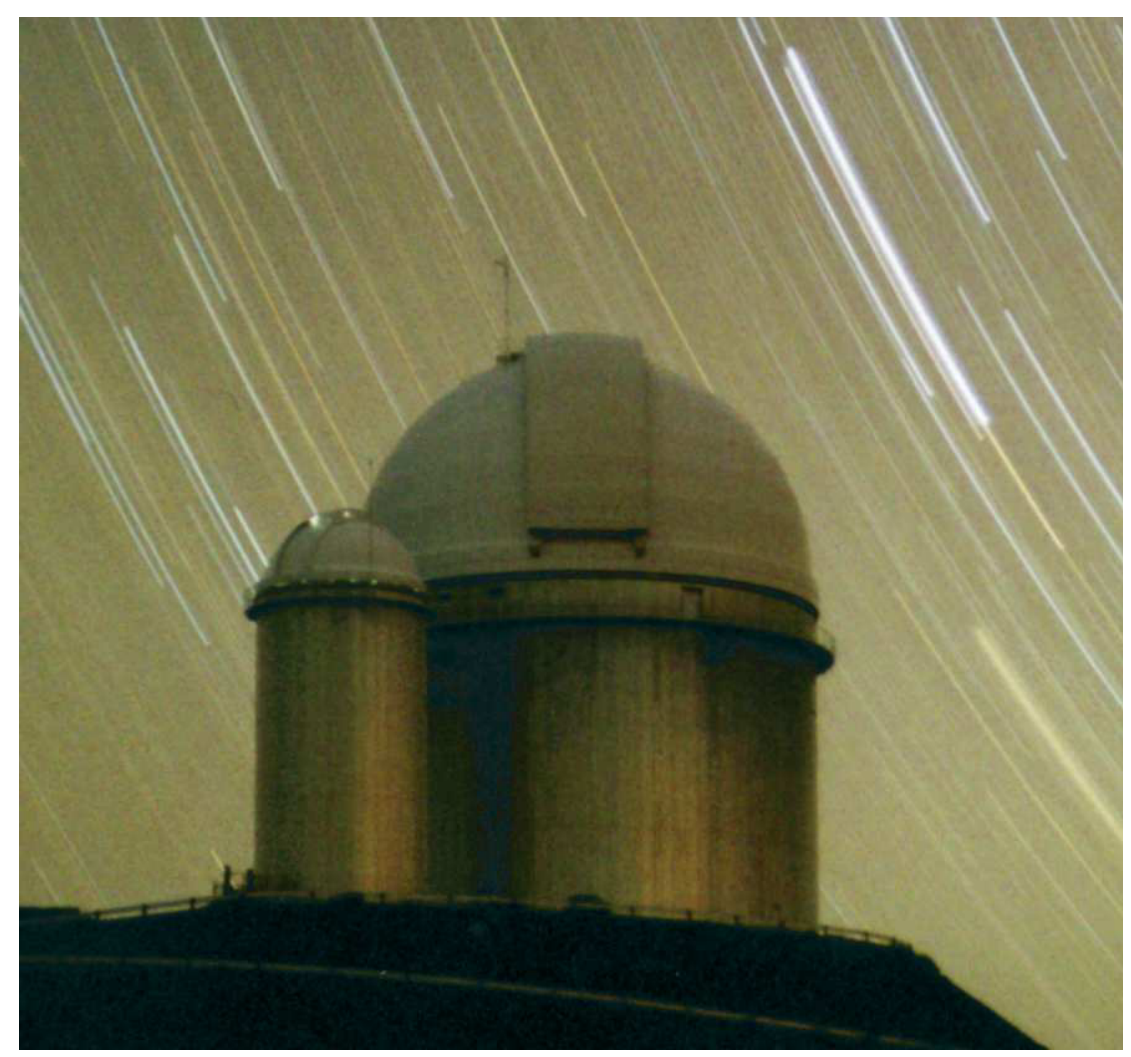



This paper was accepted October 2002

as article to Astronomy \& Astrophysics.

\title{
Tidal Dwarf Candidates in a Sample of Interacting Galaxies
}

\section{Properties and Kinematics of the Ionized Gas*}

\author{
P. M. Weilbacher ${ }^{1}$, P.-A. Duc ${ }^{2}$, U. Fritze-v. Alvensleben ${ }^{1}$ \\ ${ }^{1}$ Universitäts-Sternwarte, Geismarlandstr. 11, 37083 Göttingen, Germany, \\ \{weilbach, ufritze\}@uni-sw.gwdg.de \\ ${ }^{2}$ CNRS URA 2052 and CEA, DSM, DAPNIA, Service d'Astrophysique, Centre d'Etudes de Saclay, \\ 91191 Gif-sur-Yvette Cedex, France, paduc@cea.fr
}

\begin{abstract}
We present low-resolution spectroscopy of the ionized gas in a sample of optical knots located along the tidal features of 14 interacting galaxies and previously selected as candidates of Tidal Dwarf Galaxies (TDGs). From redshift measurements, we are able to confirm their physical association with the interacting system in almost all cases. For most knots, the oxygen abundance does not depend on the blue luminosity. The average, $12+\log (\mathrm{O} / \mathrm{H})=8.34 \pm 0.20$, is typical of TDGs and comparable to that measured in the outer stellar disk of spirals from which they were formed. A few knots showing low metallicities are probably pre-existing low-mass companions. The estimated $\mathrm{H} \alpha$ luminosity of the TDG candidates is higher than the one of typical individual $\mathrm{H}$ II regions in spiral disks and comparable to the global $\mathrm{H} \alpha$ luminosity of dwarf galaxies. We find several instances of velocity gradients with amplitudes apparently larger than $100 \mathrm{~km} \mathrm{~s}^{-1}$ in the ionized gas in the tidal knots and discuss various possible origins for the large velocity amplitudes. While we can exclude tidal streaming motions and outflows, we cannot rule out projection effects with the current resolution. The velocity gradients could be indicative of the internal kinematics characteristic of self-gravitating objects. Higher resolution spectra are required to confirm whether the tidal knots in our sample have already acquired their dynamical independence and are therefore genuine Tidal Dwarf Galaxies.
\end{abstract}

Key words: Galaxies: formation - Galaxies: interactions - Galaxies: optical spectroscopy - Galaxies: photometry

*Based on observations collected at the European Southern Observatory, La Silla, Chile (ESO No 64.N-0361). 


\section{Introduction}

The formation of Tidal Dwarf Galaxies (TDGs) in interacting galaxies is by now a well recognized phenomenon. Previous work on the subject has mostly focused on detailed, multi-wavelength analyses of individual systems (e.g. Hibbard et al. 1994, Duc et al. 1997, etc.). The first attempt to create a large sample of TDGs was presented by Weilbacher et al. (2000, hereafter Paper I), how we selected TDG candidates among optical knots in the tidal features of 10 interacting systems situated at different redshifts below $z=0.1$. The parent galaxies were selected from the catalog of (Arp \& Madore 1987) to resemble the perturbed systems observed in deep surveys of the distant universe. Evolutionary synthesis modeling was used to rule out background objects by their broad-band colors.

What makes an optical clump in tidal features a Tidal Dwarf Galaxy is still not well-defined. In several studies, the most luminous knots in or near tidal tails were classified as TDGs. However, contamination by background galaxies is not unlikely. For instance, the blue object of apparent dwarf galaxy luminosity positioned at the tip of one of the long tidal tails in the Superantennae, presented by Mirabel et al. (1991) as a TDG candidate, was found to be a background galaxy (F. Mirabel, priv. comm.). Another example is the study of Hunsberger et al. (1996), who found numerous TDG candidates in the eastern tidal tail of NGC 7319, a member of the Stephan's Quintet (HCG 92). Other studies could only confirm the association of one of these with the compact group (Xu et al. 1999, Iglesias-Páramo \& Vílchez 2001). Projection effects within a bent tidal tail could also mimic dwarf galaxysized accumulations of material. As normal dwarf galaxies are stable entities with their own dynamics, the best definition of a TDG is that it be a self-gravitating entity (Duc et al. 2000, Weilbacher \& Duc 2001). Thus, to confirm a knot in a tail as a genuine TDG, the velocity distribution within the knot has to be measured, to determine if it is decoupled from the expanding motion of the tidal tail, and possibly rotating, obviously a difficult task. Studying the dynamics of the HI gas, Hibbard et al. (2001) failed to prove that the most well known tidal dwarf galaxy candidate, discovered by Schweizer (1978) and Mirabel et al. (1992) in the southern tail of the Antennae, is actually gravitationally bound.

In this paper, we present the spectrophotometric follow-up of the photometric survey of interacting galaxies presented in Paper I. This is the first spectroscopic investigation of a sample of TDG candidates. The immediate objective of this work is to confirm the physical association of the observed tidal knots with the parent interacting system. This enables us to judge the effectiveness of a preselection of star-forming TDG candidates based only on photometric models such as the ones used in Paper I. We also obtain some key input parameters for the evolutionary synthesis modeling code, such as extinction, metallicity, and Balmer line equivalent widths. We use this data as input for the models in Paper III of this series (Weil- 
Table 15: Observing summary

\begin{tabular}{cll}
\hline Object & Observing Mode & Exposure time \\
\hline AM 0529-565 & MOS & $4 \times 1200 \mathrm{~s}$ \\
AM 0537-292 & $2 \times$ MOS & $3 \times 1200 \mathrm{~s}$ each \\
AM 0547-244 & MOS & $3 \times 1200 \mathrm{~s}$ \\
AM 0547-474 & Imaging $B, V, R$ & $500,400,300 \mathrm{~s}$ \\
& $2 \times$ Long-slit & $3 \times 1200,3 \times 480 \mathrm{~s}$ \\
AM 0607-444 & MOS & $3 \times 1200 \mathrm{~s}$ \\
AM 0748-665 & MOS & $3 \times 1200 \mathrm{~s}$ \\
AM 1054-325 & MOS & $2 \times 1200 \mathrm{~s}$ \\
& Long-slit & $2 \times 600 \mathrm{~s}$ \\
AM 1159-530 & Imaging $B, V, R$ & $500,400,300 \mathrm{~s}$ \\
& MOS & $3 \times 1200 \mathrm{~s}$ \\
AM 1208-273 & MOS & $3 \times 1200 \mathrm{~s}$ \\
AM 1237-364 & Imaging $B, V, R$ & $3 \times 200,140,110 \mathrm{~s}$ \\
& MOS & $3 \times 1200 \mathrm{~s}$ \\
AM 1324-431 & Imaging $B, V, R$ & $600,480,300 \mathrm{~s}$ \\
& MOS & $3 \times 1200 \mathrm{~s}$ \\
AM 1325-292 & Long-slit & $3 \times 600 \mathrm{~s}$ \\
AM 1353-272 & MOS & $3 \times 1200 \mathrm{~s}$ \\
\hline
\end{tabular}

bacher et al. in prep.) to determine the evolutionary status of the TDG candidates. Here, we also make a first attempt to probe the dynamical status and hence real nature of the tidal objects using long-slit velocity curves.

After describing our data acquisition and analysis methods in Sect. 2, we present the basic results for our sample of TDG candidates in Sect. 3. In Sect. 4, we discuss these results in detail and describe our method of selecting real TDGs from the sample using spectroscopic data. A summary of the results is given in Sect. 5. We present the photometric data of four new interacting systems in the appendix (App. A) along with individual notes on the spectroscopic results (App. B).

\section{Observations and data analysis}

\subsection{Spectroscopic observations}

Our sample consists of the 10 interacting systems studied in Paper I plus four additional objects extracted from the Arp \& Madore Catalogue of Southern Peculiar Galaxies (see App. A).

We observed the 14 interacting systems in January 2000 with EFOSC2 installed on the ESO $3.6 \mathrm{~m}$ telescope (see Table 15). We made use both of the multi-object 
spectroscopic mode (MOS) with 1.'7 slitlets and of the long-slit mode with a $1 . .5$ width slit. The geometry and orientations of all slits are indicated in Figs. 36 to 48. The finding charts consist of deep EFOSC2 $R$-band images (logarithmic).

The low resolution grism \#11 was used (13.2 $\AA$ FWHM resolution for a $1^{\prime \prime}$ slit), providing a spectral coverage from 3380 to $7540 \AA$ for a central slit. The detector was a LORAL CCD with $15 \mu \mathrm{m}$ pixel size $\left(0.157^{\prime \prime}\right.$ on the sky), read out binned $2 \times 2$. The weather was photometric; several spectroscopic standard stars were observed per night. The seeing varied slightly between $0 . " 85$ and $1 . " 20$. For our four new systems (AM 0547-474, AM 1159-530, AM 1237-364, and AM 1324-431, see App. A) we also obtained broad band images in $B, V, R$ and observed photometric standard stars. The reduction in these cases was done using the same procedure as in Paper I. The errors in the photometric calibration are below $0.05 \mathrm{mag}$ in all cases.

The standard reduction was done in IRAF ${ }^{1}$. We have created an IRAF task mosx to handle MOS frames semi-automatically. The task proceeds in the following manner:

1. extraction of the science slit spectra, flat-fields, and calibration HeAr spectra

2. correction of science spectra using flat-field response function

3. identification of HeAr lines and first-order wavelength calibration

4. higher order two-dimensional fit to the HeAr lines to correct for curved slits and the non-linear dispersion

5. flux-calibration of the individual science spectra

A similar procedure was used to correct the long-slit spectra. Regions of interest were then extracted from the 2D spectra and the visible emission lines were measured using IRAF's splot task. Three spectra representative for those with low, medium, and high $\mathrm{S} / \mathrm{N}$ of our sample are shown in Fig. 29.

We corrected the Balmer emission line fluxes for contributions of underlying stellar absorption. If the absorption was detected around the emission line, we fitted two Gaussians with different widths to deblend absorption and emission components of the lines. If the absorption was not visible, the we assumed a constant equivalent width of $2 \AA$ for the absorption, a standard value used by numerous authors (see e.g. Zaritsky et al. 1994, van Zee et al. 1998). The total reddening was determined from the Balmer decrement, and then applied to all line ratios to derive the corrected flux

\footnotetext{
${ }^{1}$ IRAF is written and supported by the IRAF programming group of the National Optical Astronomy Observatories (NOAO).
} 


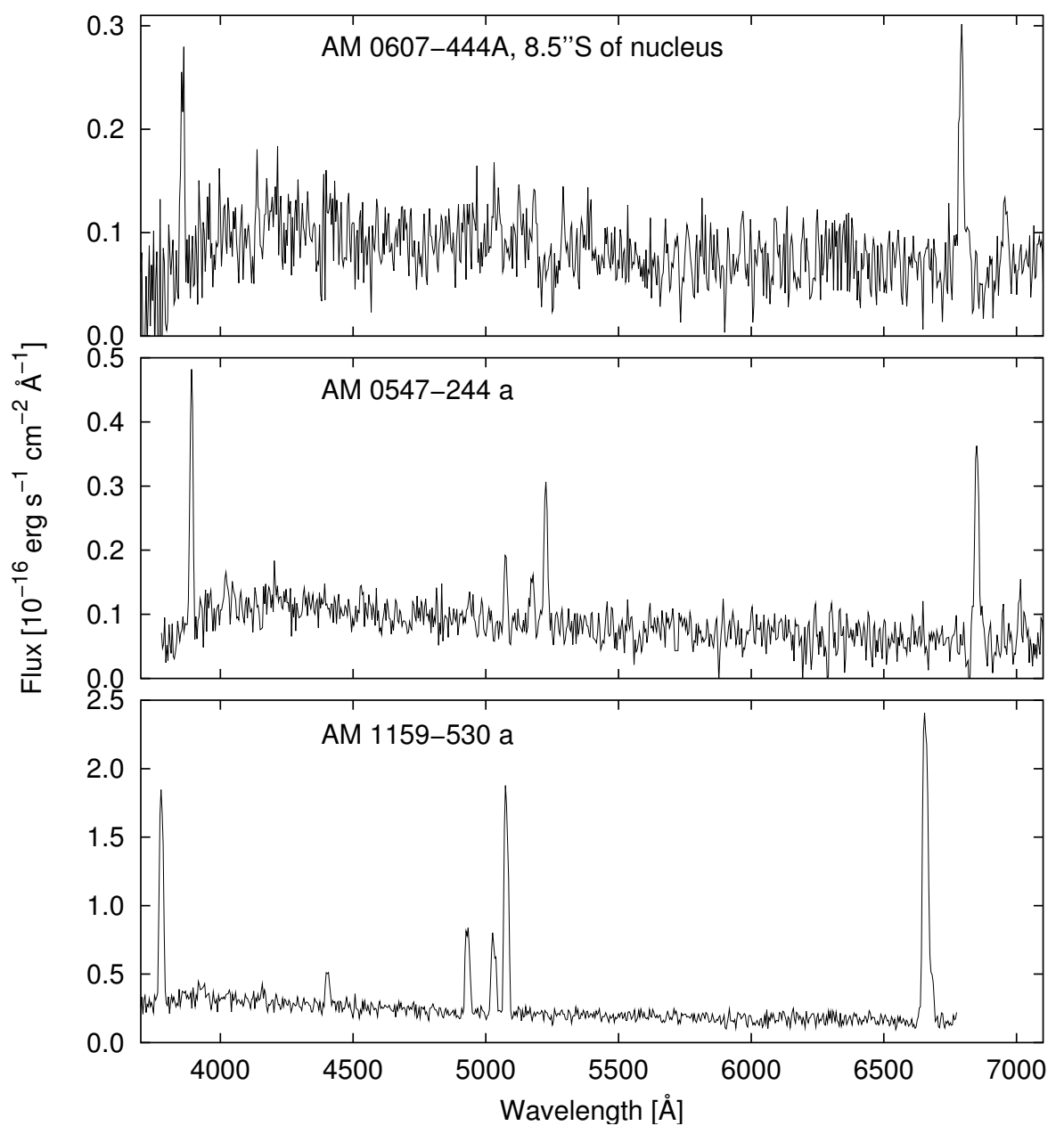

Figure 29: Typical low, medium, and high $\mathrm{S} / \mathrm{N}$ spectra of three of the observed knots. The spectra are shown with the observed wavelengths and without reddening correction.

$I$ using $I / I_{\mathrm{H} \beta}=F / F_{\mathrm{H} \beta} 10^{C f(\lambda)}$ with the reddening curve $f(\lambda)$ taken from Whitford (1958). In absence of good S/N Balmer emission lines, we used the Galactic extinction values of Schlegel et al. (1998) as given in NED ${ }^{2}$.

\footnotetext{
${ }^{2}$ The NASA/IPAC Extragalactic Database (NED) is operated by the Jet Propulsion Laboratory, California Institute of Technology.
} 
Table 16: Distance and absolute blue magnitude of the parent galaxies

\begin{tabular}{|c|c|c|c|}
\hline System & $\mathrm{D}[\mathrm{Mpc}]$ & ID & $\mathbf{M}_{B}[\mathrm{mag}]$ \\
\hline \multirow{4}{*}{ AM 0529-565 } & \multirow{4}{*}{59.7} & $\mathrm{~A}$ & -18.1 \\
\hline & & (Aw) & -15.1 \\
\hline & & $(\mathrm{Ae})$ & -15.2 \\
\hline & & $\mathrm{D}$ & -15.4 \\
\hline \multirow{2}{*}{ AM 0537-292 } & \multirow{2}{*}{52.0} & A & -18.6 \\
\hline & & B & -17.0 \\
\hline \multirow{2}{*}{ AM 0547-244 } & \multirow{2}{*}{176} & $\overline{\mathrm{A}}$ & -20.6 \\
\hline & & B & -18.3 \\
\hline \multirow{2}{*}{ AM 0547-474 } & \multirow{2}{*}{203} & A & -21.0 \\
\hline & & $\mathrm{C}$ & -19.5 \\
\hline \multirow{2}{*}{ AM 0607-444 } & \multirow{2}{*}{170} & $\mathrm{~A}$ & -20.8 \\
\hline & & (b) & -16.0 \\
\hline AM 0642-325 & 366 & $\mathrm{~A}$ & -21.4 \\
\hline \multirow{2}{*}{ АM 0748-665 } & \multirow{2}{*}{313} & $\mathrm{~A}$ & -19.5 \\
\hline & & B & -21.1 \\
\hline \multirow{2}{*}{ AM 1054-325 } & \multirow{2}{*}{52.9} & A & -19.1 \\
\hline & & $\mathrm{B}$ & -18.3 \\
\hline AM 1159-530 & 60.7 & A & -20.2 \\
\hline \multirow{2}{*}{ AM 1208-273 } & \multirow{2}{*}{166} & A & -20.5 \\
\hline & & $\mathrm{C}$ & -17.1 \\
\hline \multirow{2}{*}{ AM 1237-364 } & \multirow{2}{*}{76.1} & $\mathrm{~A}$ & -19.9 \\
\hline & & B & -16.1 \\
\hline \multirow{2}{*}{ AM 1324-431 } & \multirow{2}{*}{142} & A & -19.9 \\
\hline & & B & -18.1 \\
\hline \multirow{2}{*}{ AM 1325-292 } & \multirow{2}{*}{60.1} & $\bar{A}$ & -20.0 \\
\hline & & B & -20.5 \\
\hline \multirow{2}{*}{ AM 1353-272 } & \multirow{2}{*}{159} & A & -20.2 \\
\hline & & B & -18.1 \\
\hline
\end{tabular}

\subsection{Velocity measurements}

We derived central velocities of all objects along the slits by calculating weighted mean redshifts from measured emission lines, afterwards converted into heliocentric velocities. Distances to the parent galaxies calculated using $H_{0}=75 \mathrm{~km} \mathrm{~s}^{-1} \mathrm{Mpc}^{-1}$ are given in Table 16 together with the absolute blue magnitudes.

Of the 36 objects selected as TDG candidates in Paper I, we obtained spectrophotometric data for 30 and determined the redshifts of 24. The inferred velocities are very close to those of the parent systems (mean velocity difference of $80 \mathrm{~km} \mathrm{~s}^{-1}$, and never exceeding a difference of $350 \mathrm{~km} \mathrm{~s}^{-1}$ ) and therefore indeed imply asso- 
ciation. For six objects where we did not detect emission lines (despite their blue color) no redshift could be measured. Their low surface brightnesses did not allow us to detect any absorption lines, either.

Among the 7 new TDG candidates presented in this paper (see App. A), four exhibit emission lines at the same velocity as the main system, one has a featureless spectrum, and two were not observed spectroscopically.

Whenever gradients were visible on the 2D spectra, we tried to derive the velocity profiles along the slit by fitting the lines with Gaussians using the IRAF task fitprofs. We used the HeAr line closest to the position in the corresponding wavelength calibration frame to correct for residual distortion. The velocity profiles obtained along a given slit with the brightest emission lines were then compared. Those that were inconsistent or had too large fitting-errors were excluded from the analysis. The final velocity curve was obtained by averaging the line velocities measured for each pixel.

Note that, given the pixel scale and seeing, the velocity measurements along the slit are not independent. Our low resolution spectra did not allow us to get a precision better than $\pm 30 \mathrm{~km} \mathrm{~s}^{-1}$ for the combined fits of lines with the highest $\mathrm{S} / \mathrm{N}$.

\subsection{Oxygen abundance measurements}

The vast majority of the emission line regions detected in our spectra are characterized by flux line ratios typical of H II regions (see Fig. 30). A clear substantial contribution by shock ionization (Dopita et al. 2000) is only observed towards several regions of AM 1054-325A, including its nucleus.

We estimated the oxygen abundances in all confirmed $\mathrm{H}$ II regions using several methods proposed in the literature. For a few objects with a tentative detection of the faint, temperature sensitive, [O III]4363 line, we could get a physical determination of the oxygen abundance (Shields 1990). However, because of its low S/N and uncertain deblending with the close $\mathrm{H} \gamma$ line, the resulting value can only be regarded as a lower limit to the actual abundance in most cases. We therefore had to rely on empirical methods such as the popular $R_{23}$, which uses the measurement of the lines [O III $] 5007+[\mathrm{O}$ III $] 4959+[\mathrm{O}$ II $] 3727$ normalized to the $\mathrm{H} \beta$ flux. The error of this method is of the order of 0.1 dex (Pilyugin 2000). A similar method is the "excitational" method of Edmunds \& Pagel (1984), which empirically relates the ratio [O III $] 5007+[\mathrm{O}$ III] 4959 to $\mathrm{H} \beta$ to the oxygen abundance. Unfortunately, both methods are degenerate: for a given value of $R_{23}$, two abundances are possible. In order to choose between the lower and higher values, we relied on the [N II]/[O II] indicator (van Zee et al. 1998). Pilyugin $(2000,2001)$ recently proposed a new method based on a complex combination of the [O III] and [O II] line fluxes called the " $P$-method". This calibration better matches the the oxygen abundances determined with the physical method, especially for high metallicity H II regions. We 

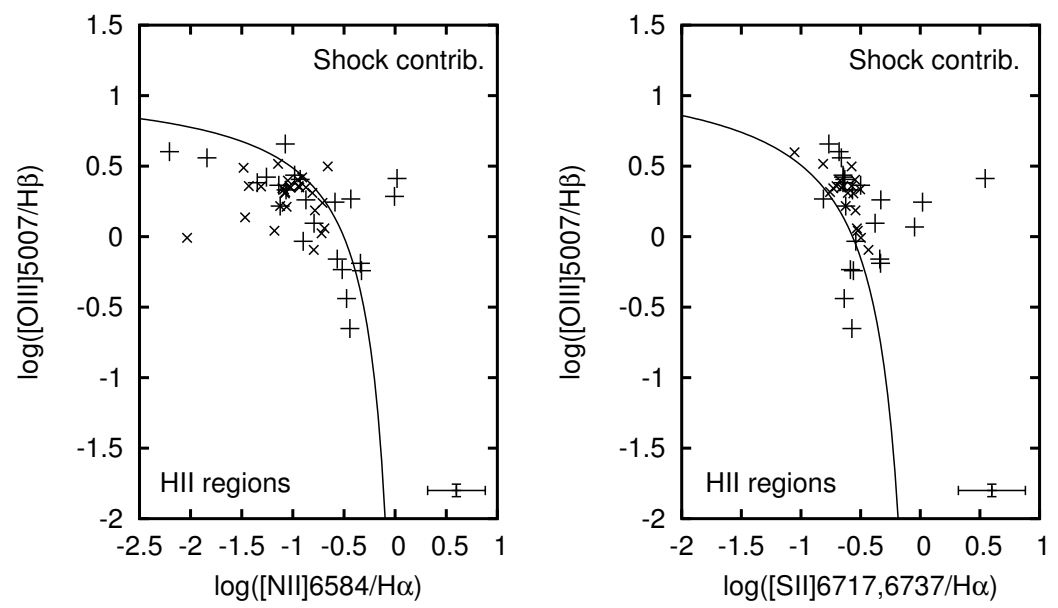

Figure 30: Physical properties of the emission line regions in our sample. The flux line ratios are indicated. The solid lines give the limit for ionization by a zero age starburst (following Dopita et al. 2000). Large pluses are regions in the parent galaxies, small crosses denote the knots. The typical error for the data points is shown in the lower right corner.

assigned a systematic error of 0.05 dex to the $P$-method for the upper branch of the $P-[\mathrm{O} / \mathrm{H}]$, and a slightly larger error of 0.08 dex for the lower branch (called $P_{3}$ ). Finally the $N$-calibration proposed by van Zee et al. (1998), based on the flux-ratio of $\mathrm{H} \alpha$ to $[\mathrm{N}$ II] 6548,6584, provides another empirical method of deriving the oxygen abundance, but suffers from deblending problems in our low resolution spectra; its uncertainty is as high as 0.2 dex.

We compared the values obtained by each of these methods and checked the consistency of the results. Because of its smaller intrinsic uncertainty, we decided to preferentially opt for the value given by the $P$-method unless the observed parameter $P$ was outside its validity range. The $N$-calibration was only used when no other methods yielded reliable results. The final oxygen abundances are listed in Table 17. The errors take into account the errors of the flux measurements plus the systematic errors of the calibrations.

\section{Results}

The individual results for each system are summarized graphically: Figs. 36 to 48 show the optical images together with several observed position-velocity diagrams 
Table 17: Line ratios, extinction values, and oxygen abundances of all observed knots.

\begin{tabular}{|c|c|c|c|c|c|c|c|}
\hline knot & $\begin{array}{c}F(\mathrm{H} \beta) \\
{\left[10^{-15} \mathrm{erg} \mathrm{s}^{-1} \mathrm{~cm}^{-2}\right.}\end{array}$ & $\mathrm{C}^{1}$ & {$[\mathrm{O}$ II $] 3727$} & $\begin{array}{r}{[\mathrm{O} \text { III }] 5007} \\
{[I(\text { line }}\end{array}$ & $\begin{array}{l}{[\mathrm{N} \text { II }] 6584} \\
/ / I(\mathrm{H} \beta)]\end{array}$ & {$[\mathrm{S} \mathrm{II}] 6717,31$} & $\begin{array}{l}\text { Oxygen } \mathrm{Ab}^{2} \\
12+\log (\mathrm{O} / \mathrm{H})\end{array}$ \\
\hline AM 0529-565D & $3.61 \pm 0.05$ & 0.07 & - & $3.97 \pm 0.28$ & - & $0.24 \pm 0.02$ & $\mathbf{7 . 6 1} \pm 0.20(\mathrm{ex})$ \\
\hline AM $0529-565 \mathrm{e}$ & $0.12 \pm 0.04$ & 0.59 & $6.19 \pm 0.38$ & $2.29 \pm 0.62$ & $0.14 \pm 0.28$ & - & $8.39 \pm 0.16(\mathrm{ex})$ \\
\hline AM 0537-292a & $0.86 \pm 0.03$ & 0.05 & $3.25 \pm 0.05$ & $2.30 \pm 0.05$ & $0.38 \pm 0.03$ & $0.89 \pm 0.04$ & $8.32 \pm 0.05(\mathrm{P})$ \\
\hline AM 0537-292 (5."0 S of b) & $0.11 \pm 0.03$ & 0.05 & $2.29 \pm 0.20$ & $1.73 \pm 0.20$ & $0.41 \pm 0.25$ & - & $8.50 \pm 0.07(\mathrm{P})$ \\
\hline AM $0537-292 c$ & $1.25 \pm 0.04$ & 0.68 & $3.63 \pm 0.04$ & $2.29 \pm 0.04$ & $0.34 \pm 0.04$ & $0.79 \pm 0.09$ & $8.26 \pm 0.05(\mathrm{P})$ \\
\hline AM $0537-292 c(N)$ & $0.77 \pm 0.03$ & 0.50 & $3.42 \pm 0.04$ & $2.03 \pm 0.05$ & $0.26 \pm 0.06$ & $0.87 \pm 0.12$ & $8.31 \pm 0.05(\mathrm{P})$ \\
\hline AM 0537-292d & $0.35 \pm 0.03$ & 0.45 & $4.27 \pm 0.14$ & $2.17 \pm 0.20$ & $0.31 \pm 0.11$ & $1.18 \pm 0.35$ & $8.20 \pm 0.06(\mathrm{P})$ \\
\hline AM 0537-292g & $0.53 \pm 0.04$ & 0.44 & $3.80 \pm 0.09$ & $2.27 \pm 0.10$ & $0.14 \pm 0.10$ & $0.63 \pm 0.18$ & $8.24 \pm 0.05(\mathrm{P})$ \\
\hline AM 0537-292h & $0.11 \pm 0.05$ & 0.48 & $5.44 \pm 0.41$ & - & $0.28 \pm 0.28$ & - & $8.23 \pm 0.39(\mathrm{~N})$ \\
\hline AM 0547-244A,br. knot & $1.92 \pm 0.06$ & 0.19 & $2.27 \pm 0.03$ & $1.14 \pm 0.04$ & $0.54 \pm 0.03$ & $0.77 \pm 0.04$ & $8.52 \pm 0.05(\mathrm{P})$ \\
\hline AM 0547-244A,E tail & $0.17 \pm 0.03$ & 0.05 & $2.41 \pm 0.11$ & $0.98 \pm 0.12$ & $0.02 \pm 0.03$ & $0.78 \pm 0.21$ & $8.50 \pm 0.06(\mathrm{P})$ \\
\hline AM 0547-244A,o.Et. & $0.14 \pm 0.03$ & 0.10 & $1.77 \pm 0.16$ & $1.37 \pm 0.16$ & $0.09 \pm 0.06$ & - & $8.60 \pm 0.07(\mathrm{P})$ \\
\hline AM 0547-244a & $0.17 \pm 0.03$ & 0.68 & $3.94 \pm 0.12$ & $2.04 \pm 0.21$ & $0.45 \pm 0.04$ & $0.72 \pm 0.23$ & $8.23 \pm 0.06(\mathrm{P})$ \\
\hline AM $0547-244 b$ & $0.39 \pm 0.03$ & 0.83 & $4.86 \pm 0.09$ & $2.53 \pm 0.10$ & $0.35 \pm 0.04$ & $0.89 \pm 0.30$ & $8.39 \pm 0.15(\mathrm{ex})$ \\
\hline AM $1054-325 \mathrm{~g}$ & $1.72 \pm 0.08$ & 0.25 & $2.05 \pm 0.03$ & $2.22 \pm 0.03$ & $0.28 \pm 0.02$ & $0.64 \pm 0.05$ & $8.48 \pm 0.05(\mathrm{P})$ \\
\hline AM $1054-325 \mathrm{~h}(\mathrm{~N})$ & $0.64 \pm 0.05$ & 0.41 & $3.39 \pm 0.09$ & $2.07 \pm 0.16$ & $0.25 \pm 0.05$ & $0.52 \pm 0.07$ & $8.28 \pm 0.05(\mathrm{P})$ \\
\hline AM $1054-325 h(S)$ & $0.32 \pm 0.05$ & 0.29 & $2.92 \pm 0.14$ & $2.24 \pm 0.17$ & $0.37 \pm 0.13$ & $0.57 \pm 0.09$ & $8.24 \pm 0.06(\mathrm{P})$ \\
\hline AM 1054-325i & $1.79 \pm 0.07$ & 0.44 & $2.22 \pm 0.03$ & $3.28 \pm 0.05$ & $0.21 \pm 0.04$ & $0.45 \pm 0.06$ & $\mathbf{7 . 7 6} \pm 0.10\left(\mathrm{R}_{23}\right)$ \\
\hline AM $1054-325 j$ & $4.37 \pm 0.07$ & 0.32 & $3.08 \pm 0.02$ & $2.28 \pm 0.02$ & $0.29 \pm 0.02$ & $0.63 \pm 0.03$ & $8.55 \pm 0.05(\mathrm{P})$ \\
\hline AM $1054-3250$ & $7.82 \pm 0.12$ & 0.47 & $2.41 \pm 0.01$ & $2.20 \pm 0.01$ & $0.24 \pm 0.01$ & $0.58 \pm 0.01$ & $8.45 \pm 0.05(\mathrm{P})$ \\
\hline AM 1159-530a (E) & $0.87 \pm 0.03$ & 0.78 & $2.71 \pm 0.05$ & $2.23 \pm 0.06$ & $0.46 \pm 0.05$ & - & $8.40 \pm 0.05(\mathrm{P})$ \\
\hline AM 1159-530a (W) & $1.28 \pm 0.05$ & 0.56 & $2.58 \pm 0.04$ & $2.63 \pm 0.07$ & $0.43 \pm 0.04$ & - & $8.40 \pm 0.05(\mathrm{P})$ \\
\hline AM 1159-530q & $0.39 \pm 0.05$ & 1.03 & $2.27 \pm 0.11$ & $1.54 \pm 0.17$ & $0.81 \pm 0.11$ & $1.41 \pm 0.31$ & $8.51 \pm 0.06(\mathrm{P})$ \\
\hline AM $1208-273 e$ & $0.22 \pm 0.03$ & 1.75 & $3.54 \pm 0.15$ & $0.80 \pm 0.15$ & $0.42 \pm 0.06$ & $0.96 \pm 0.30$ & $8.33 \pm 0.07(\mathrm{P})$ \\
\hline AM 1208-273h & $0.28 \pm 0.04$ & 0.87 & $3.64 \pm 0.14$ & $1.32 \pm 0.14$ & $0.26 \pm 0.03$ & $0.67 \pm 0.17$ & $8.31 \pm 0.06(\mathrm{P})$ \\
\hline AM 1208-273i & $0.21 \pm 0.02$ & 0.13 & $2.51 \pm 0.11$ & $1.10 \pm 0.09$ & $0.17 \pm 0.03$ & $0.77 \pm 0.15$ & $8.48 \pm 0.06(\mathrm{P})$ \\
\hline AM 1237-364d & $0.15 \pm 0.04$ & 0.14 & $3.08 \pm 0.24$ & $3.15 \pm 0.35$ & $0.74 \pm 0.09$ & $0.89 \pm 0.13$ & $8.30 \pm 0.07(\mathrm{P})$ \\
\hline AM $1237-364 \mathrm{e}$ & $0.16 \pm 0.02$ & 0.14 & $2.85 \pm 0.15$ & $1.65 \pm 0.20$ & $0.19 \pm 0.11$ & $0.60 \pm 0.15$ & $8.41 \pm 0.06(\mathrm{P})$ \\
\hline AM $1237-364 \mathrm{~g}$ & $2.28 \pm 0.06$ & 0.36 & $1.92 \pm 0.03$ & $3.08 \pm 0.04$ & $0.10 \pm 0.03$ & - & $\mathbf{7 . 7 2} \pm 0.05$ (phys) \\
\hline AM 1324-431c & $0.12 \pm 0.06$ & 0.21 & $2.82 \pm 0.34$ & $1.09 \pm 0.29$ & $0.66 \pm 0.16$ & - & $8.66 \pm 0.22(\mathrm{~N})$ \\
\hline
\end{tabular}

${ }^{1}$ Total extinction from the Balmer decrement.

${ }^{2}$ In brackets we list the method used to derive the oxygen abundance as described in Sect. 2.3.

and derived velocity curves. All objects and knots discussed are labeled; the slits are overplotted in their real scale (length and width). Measured mean velocities or redshifts are indicated. Low metallicity objects with $12+\log (\mathrm{O} / \mathrm{H})<8.0$ are circled and objects in the slits without firmly detected lines are marked "n.d.". More detailed descriptions of each system may be found in Paper I and in App. B.

Below, we present the properties of the objects that proved to be physically associated with the tidal features. We concentrate our analysis on the emission-line 


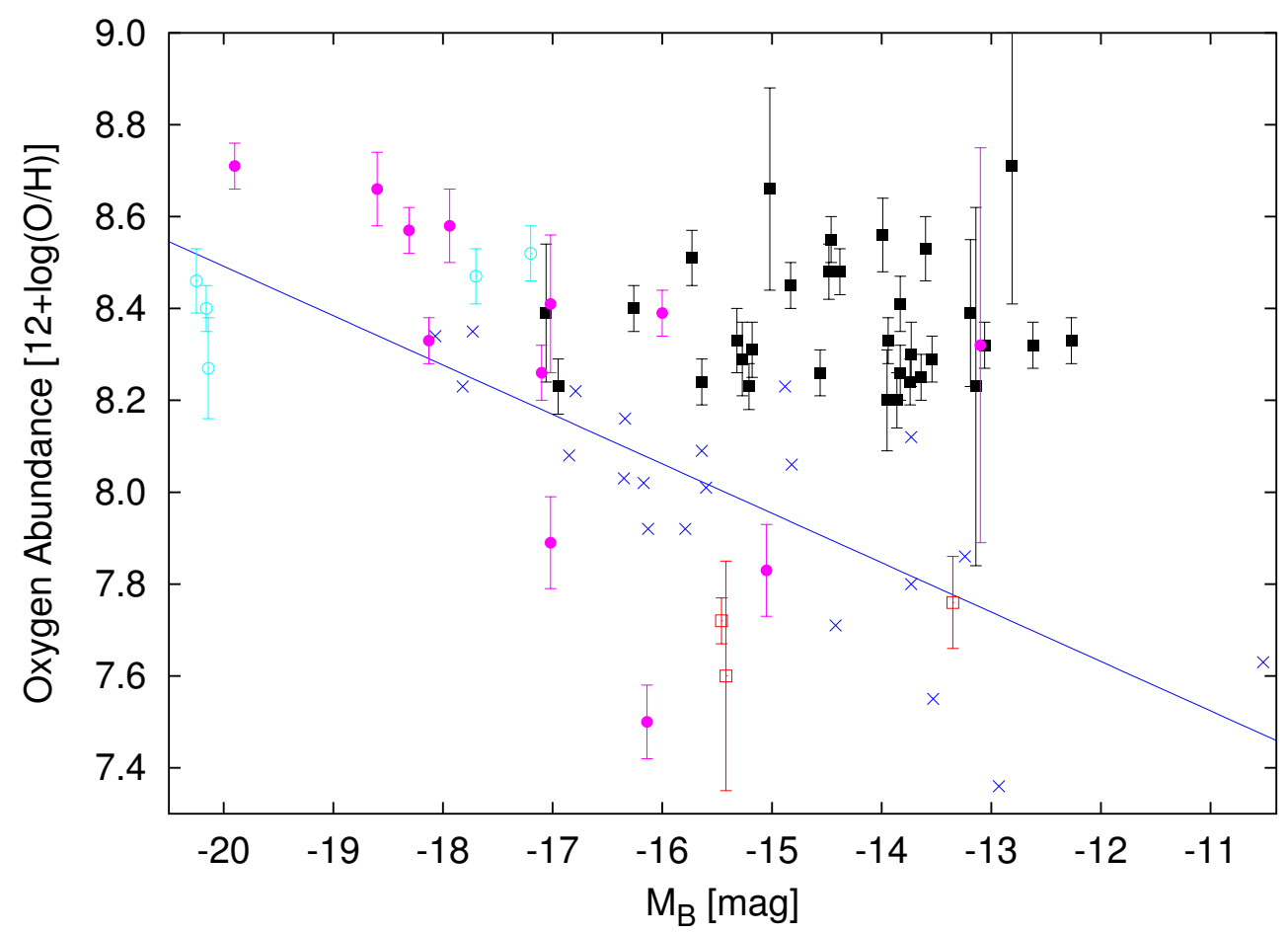

Figure 31: Metallicity-luminosity relation for different types of objects: local isolated dwarf galaxies (crosses), knots in the tidal features (filled/open squares), main group members (filled circles), background objects (open circles).

regions that they host. Our selection of TDG candidates was biased towards starforming objects (see Paper I) and, indeed, the vast majority of them host H II regions.

\subsection{Metallicity}

The metallicity-luminosity relation for the objects in our sample and for isolated dwarf galaxies is shown in Fig. 31. Nearby dwarf irregular galaxies (Richer \& McCall 1995) follow the well-known correlation indicated by the linear fit. The massive galaxies in our sample - main galaxies and background objects - also follow this relation ${ }^{3}$. On the other hand, knots along the tidal features deviate significantly from the relation and seem to have a metallicity which is independent of luminosity. The bulk of the emission-line knots in our sample have $12+\log (\mathrm{O} / \mathrm{H})$ in the

\footnotetext{
${ }^{3}$ The exceptions are two low metallicity star-bursting dwarf galaxies (AM 1237-364 B and C), the western nucleus 'Aw' of AM 0529-565, and one low surface brightness galaxy near the interacting system AM 1353-272.
} 
range $8.3 \ldots 8.7$ (mean $12+\log (\mathrm{O} / \mathrm{H})=8.34 \pm 0.14 \approx 1 / 4 \odot$ ) and luminosities $M_{B}=-12 \cdots-17 \mathrm{mag}$. They occupy the position of previously published TDGs (see e.g. Duc et al. 2000). Three objects, AM 0529-565 D, AM 1237-364 g, and AM 1054-325 i, however, have oxygen abundances at least 0.4 dex lower than the rest of the knots.

\subsection{Internal extinction}

In knots where we could determine internal extinction from the Balmer decrement in addition to the Galactic absorption, we derive $A_{V}$ in the range $0.0 \ldots 3.3$ mag with a mean of $A_{V}=0.81 \mathrm{mag}$.

This is roughly half the mean extinction and range observed in $\mathrm{H}$ II regions in spiral galaxies (e.g. mean $A_{V}=3.1 \mathrm{mag}$ and $0<A_{V}<6$ mag for $\mathrm{H}$ II regions in the central part of M51, Scoville et al. 2001). This is understandable as our slits sample a much larger field (typically several hundreds of parsecs, see Sect. 4.1) than the spatially resolved HST imaging studies of Scoville et al. (typically a few parsecs). Our slits therefore average over several individual $\mathrm{H}$ II regions and the regions between them. As the extinction is typically much higher within $\mathrm{H}$ II regions where the dust accompanies the star formation process, this naturally explains the smaller range and smaller mean value we see in our sample.

Our mean extinction is also comparable to the typical $A_{V} \simeq 1.0 \mathrm{mag}$ of the young star clusters in the less obscured regions of the Antennae (Whitmore et al. 1999).

\subsection{H $\alpha$ luminosities}

Other authors before have used the H $\alpha$ luminosity to discriminate between candidates for TDGs and normal H II regions (see e.g. Iglesias-Páramo \& Vílchez 2001). We have compiled the $\mathrm{H} \alpha$ luminosities of all the tidal knots studied in this survey. Note that the raw luminosities (as e.g. presented in Table 17) are lower limits since they are measured with an aperture-limited slit and not from narrow band imaging. From the size of the optical knots as measured on $B$-band images and the area covered by the slit, we have derived correction factors between 1.5 and 5 depending on the coverage of the knot by the slit and used these to estimate the total $\mathrm{H} \alpha$ luminosities of the knots. The average $\mathrm{H} \alpha$ luminosity we derive is $2.2 \cdot 10^{39} \mathrm{erg} \mathrm{s}^{-1}$. How does this value compare with that measured in typical $\mathrm{H}$ II regions of disk galaxies and other dwarf galaxies?

A histogram of the $\mathrm{H} \alpha$ luminosity distribution of the tidal knots is shown in Fig. 32 where it is compared to the luminosity function (LF) of H II regions in four nearby galaxies of various morphological types (the LMC, NGC 2403, M 101, and NGC 3310). The data was extracted from the catalog of Kennicutt et al. (1989), compiled from ground-based narrow band images. This plot shows an apparent 


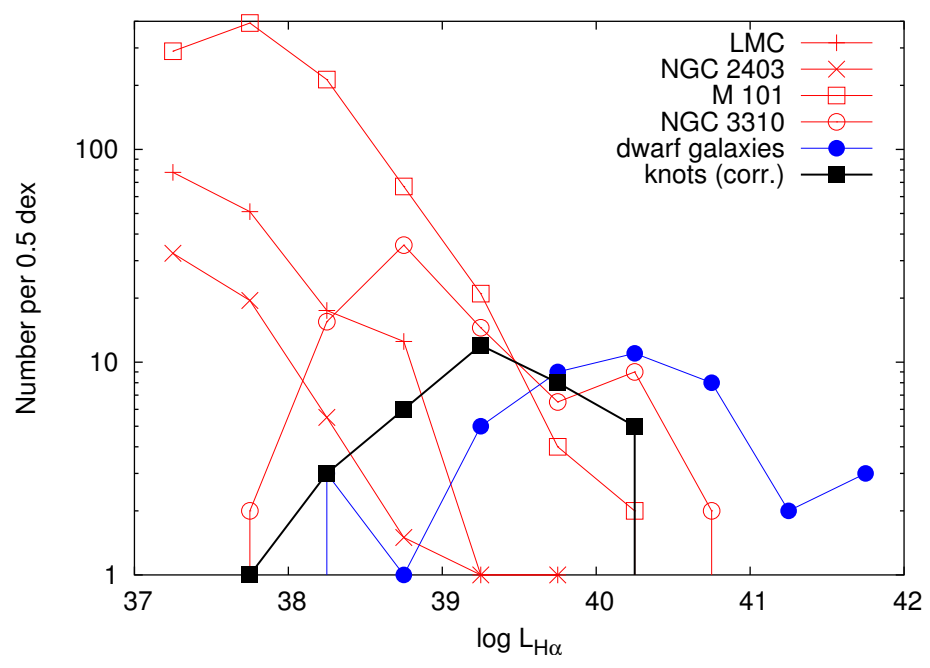

Figure 32: H $\alpha$ luminosity distribution of the observed knots with confirmed higher metallicity compared with literature values for individual $\mathrm{H}$ II regions in spiral disks and total blue dwarf galaxies.

large diversity in the shapes of the H II LFs. All individual H II regions in the LMC and the Sc spiral NGC 2403 have H $\alpha$ luminosities about one order of magnitude below the most luminous knots in our survey $\left(\sim 10^{40} \mathrm{erg} \mathrm{s}^{-1}\right)$. The spiral M 101 hosts a few giant $\mathrm{H}$ II regions reaching this value. The LF of the more distant galaxy NGC $3310(\mathrm{D} \simeq 19 \mathrm{Mpc})$ is affected by incompleteness for $\log L(\mathrm{H} \alpha)<38.5$ and has a high number of luminous $\mathrm{H}$ II regions with a distribution very similar to that of our knots. This galaxy is very perturbed and probably has recently undergone a merger.

In the same Figure we also present a comparison with the global $\mathrm{H} \alpha$ luminosity distribution of "normal" dwarf galaxies consisting of $11 \mathrm{Im}$ and $7 \mathrm{dI}$ galaxies of Gallagher et al. (1989), 12 nearby "amorphous" dwarf galaxies of Marlowe et al. (1997), and 12 blue compact dwarfs of Cairós et al. (2001). The knots have a distribution similar to the low luminosity end of the normal dwarfs, while there are several dwarf galaxies with luminosities one order of magnitude higher than any of the knots in the tidal tails.

\subsection{Kinematics}

Due to observational constraints, we could only partially sample the velocity curves along the tails from our long-slit and MOS spectra. A striking result from this study is the presence of kinematical structures with large apparent velocity gradients along the tidal tails. 
Table 18: Observed velocity gradients

\begin{tabular}{|c|c|c|c|c|}
\hline TDG candidate & $\begin{array}{l}\Delta V_{\max } \\
{\left[\mathrm{km} \mathrm{s}^{-1}\right]}\end{array}$ & $\begin{array}{c}R \\
{[\mathrm{kpc}]}\end{array}$ & $\begin{array}{c}\text { Angle } \\
{\left[{ }^{\circ}\right]}\end{array}$ & $\begin{array}{l}\text { Extent } \\
\qquad \text { (seeing) }\end{array}$ \\
\hline AM 0537-292a & $70 \ldots 360 \pm 50$ & 8.10 & 43 & $1 . " 5 / 2 ! .7(1 . " 04)$ \\
\hline AM $0537-292 \mathrm{~g}$ & $160 \pm 50$ & 12.60 & 83 & $2 ! 9(1.26)$ \\
\hline AM 0547-244a & $77 \pm 61$ & 23.60 & 5 & 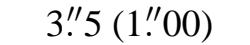 \\
\hline AM 0547-244b & $220 \ldots 485 \pm 80$ & 30.90 & 85 & 2."4/4."4 (1."00) \\
\hline AM 1054-325h & $130 \ldots 420 \pm 45$ & 6.40 & 2 & $2 . " 0 / 3 . ! 5(1 . " 00)$ \\
\hline AM 1159-530a & $440 \pm 45$ & 29.30 & 29 & 5."5 (1.!04) \\
\hline AM $1353-272 a^{*}$ & $343 \pm 17$ & 39.30 & 0 & $3 !^{\prime \prime} 0\left(1 !^{\prime \prime} 02\right)$ \\
\hline AM $1353-272 b^{*}$ & $87 \pm 18$ & 33.70 & 0 & $2 .^{\prime \prime} 6(1$. \\
\hline AM $1353-272 c^{*}$ & $34 \pm 14$ & 31.60 & 0 & $3 !^{\prime \prime} 2(1 . \prime 02)$ \\
\hline AM $1353-272 d^{*}$ & $93 \pm 9$ & 28.10 & 0 & 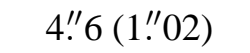 \\
\hline AM $1353-272 k^{*}$ & $50 \pm 7$ & 22.30 & 0 & $2 ! " 2(1 . " 02)$ \\
\hline AM $1353-2721^{*}$ & $24 \pm 6$ & 24.70 & 0 & 2 ".6 (1."02) \\
\hline AM $1353-272 m^{*}$ & $43 \pm 8$ & 28.30 & 0 & 3."4 (1."02) \\
\hline
\end{tabular}

*observed with FORS2 at the VLT (Weilbacher et al. 2002)

In our low resolution data, we find several instances of knots with discrepant velocities. In a few cases, we have enough spatial resolution to measure apparent velocity gradients at the location of optically detected objects: in AM0537292 (Fig. 38), AM 0547-244 (Fig. 39), AM 1054-325 (Fig. 43), and AM 1159-530 (Fig. 44). The apparent velocity differences $\Delta V_{\max }$ between two close regions can be as high as several hundred $\mathrm{km} \mathrm{s}^{-1}$. In three knots (AM 0537-292a, AM 0547-244b, and AM 1054-325h) we observe substructure even with our low spatial resolution. In these cases, we note the total amplitude of the gradient and $\Delta V_{\max }$ within the brighter knot.

All observed velocity gradients are summarized in Table 18. There, we also list the distance $R$ between the knot in the tidal tail and the nucleus of the parent galaxy along the tail. We also give the angle between the orientation of the slit and the central ridge of the tail, and the extent of the observed velocity gradient.

In Figs. 33 to 35, parameters which might affect the strength of the velocity gradient given by the $\Delta V_{\max }$ variable are plotted. Fig. 33 shows the maximum velocity difference versus the absolute blue magnitude. We do not observe any obvious trend between the stellar mass very - roughly represented by the blue luminosity - and the dynamical mass to which $\Delta V_{\max }$ is presumably a rough indicator. In Fig. 34, we see that $\Delta V_{\max }$ of the knots does not depend on the distance to the nucleus of the parent galaxy: small gradients occur both near to and far away from the parent nucleus. In Fig. 35, we investigate if the angle between the slit and the tail has a significant contribution to the value of the velocity gradient. As the observations of 


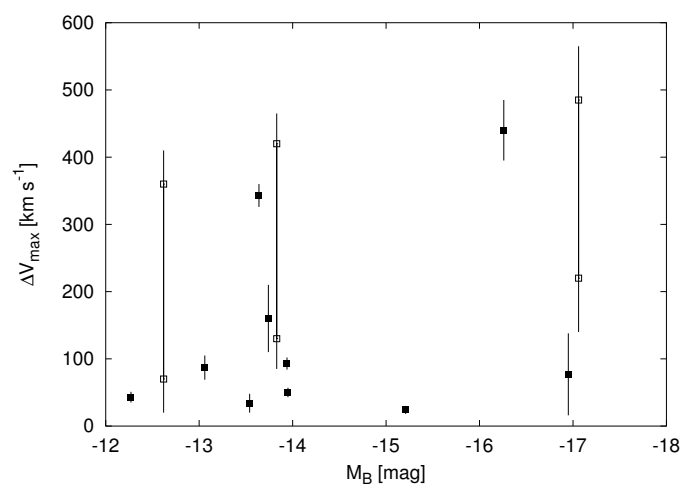

Figure 33: Maximum velocity difference $\Delta V_{\max }$ vs. absolute blue magnitude of our TDGs. Filled squares mark TDGs with only one possible $\Delta V_{\max }$, the three cases with two possible values of $\Delta V_{\max }$ have been plotted as 2 connected open squares to visualize the possible range in $\Delta V_{\max }$.

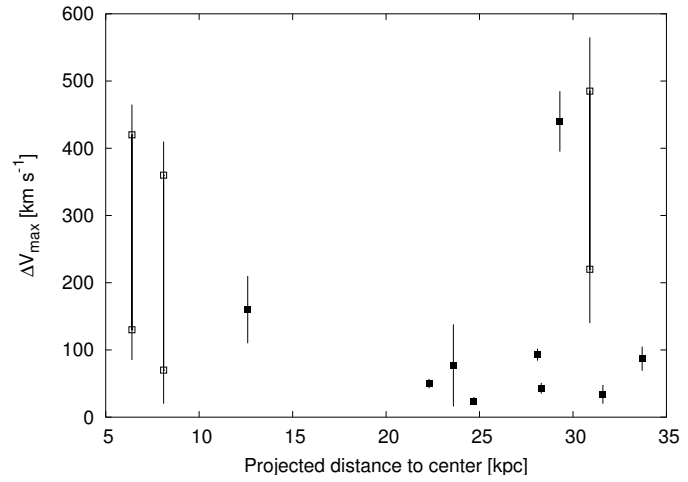

Figure 34: Maximum velocity difference $\Delta V_{\max }$ vs. projected distance nucleusknot along the tail. Symbols as in Fig. 33.

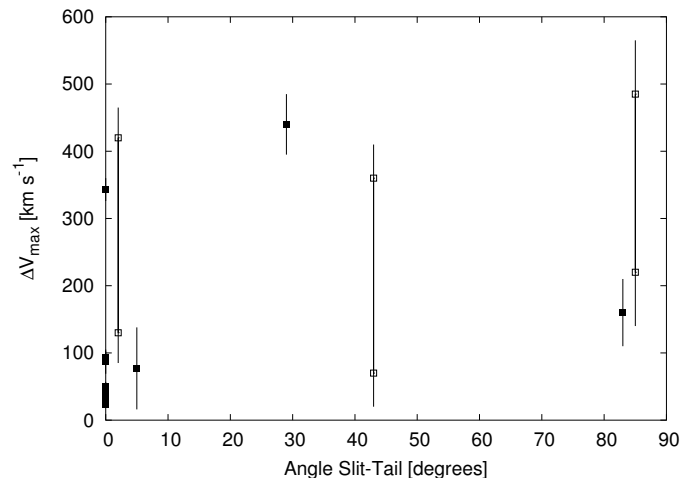

Figure 35: Maximum velocity difference $\Delta V_{\max }$ vs. angle slit-tail. Symbols as in Fig. 33 . 
AM 1353-272 with its 7 knots were performed with slits along the tails (Weilbacher et al. 2002), we have a strong selection effect towards low angles, and cannot derive a dependence between the angle and $\Delta V_{\max }$. However, we note that velocity gradients are observed at all angles between the slit and the tail. That means that we see velocity components both along and perpendicularly to the tail.

\section{Discussion}

The tidal knots we observed appear to have rather unusual characteristics: in particular their large $\mathrm{H} \alpha$ luminosities, and the kinematics of their ionized gas, with velocity amplitudes greater than $100 \mathrm{~km} \mathrm{~s}^{-1}$ are striking. With such properties, the TDG candidates in our sample appear to be closer to individual dwarf galaxies than to the giant HII regions of spiral disks.

\subsection{TDG candidates: between giant HII regions and individual dwarfs galaxies?}

\section{H $\alpha$ luminosities}

What can we learn from the comparison with the H II region luminosities? The spatial coverage of our slits is typically $3 !^{\prime \prime} 5 \times 1{ }^{\prime \prime} \cdot 7$ (the extraction width times the slit width for MOS $)$. For the nearby systems $(D \sim 50 \mathrm{Mpc})$ we therefore summed the $\mathrm{H} \alpha$ flux over a region of $850 \times 410 \mathrm{pc}^{2}$; for a distance of $150 \mathrm{Mpc}$ this would increase to $2.5 \times 1.2 \mathrm{kpc}^{2}$. From this rough estimate is seems clear that we do not observe individual $\mathrm{H}$ II regions, but cover a region much larger in size. With the value found for M 101 by Pleuss et al. (2000) a minimum distance between H II regions is 60 pc; then about 100 individual H II regions would "fit" into the region we sample for our nearby systems and 1000 for the distant ones. With a typical individual luminosity of $10^{37} \mathrm{erg} \mathrm{s}^{-1}$ (Scoville et al. 2001), one would then expect integrated luminosities of $10^{39} \ldots 10^{40} \mathrm{erg} \mathrm{s}^{-1}$, similar to the upper end of the luminosity distribution of the knots. The luminous end of our knots will then be the real starbursts in our sample, while the faint end are regions forming stars at a lower level.

The comparison with the sample of dwarf galaxies is more difficult, because several of the objects shown in Fig. 32 are not classical dwarf galaxies with $M_{B}<-17$ mag but have higher blue luminosities. The $\mathrm{H} \alpha$ luminosity does not linearly depend on the star formation rate for dwarf galaxies, but is strongly affected by evolutionary effects as shown by Weilbacher \& Fritze-von Alvensleben (2001). This does not allow us to draw any conclusions about the star formation rates of both types of objects. In summary, the H $\alpha$ luminosities emitted by the tidal knots appear to be lower than the global $L(\mathrm{H} \alpha)$ of normal galaxies with $M_{B} \leq-19$ mag. However, 
they are at least similar to and in most cases exceed those measured in $\mathrm{H}$ II regions located in the disks of nearby spiral galaxies. It seems clear that the knots we observe are actually a conglomerate of several individual $\mathrm{H}$ II regions. If that is the case, one may wonder whether they do belong to a single dynamical object.

\section{Metallicities}

Currently known TDGs have metallicities around 1/3 solar (Duc et al. 2000), significantly higher than normal dwarf galaxies of similar luminosity. This can be understood if TDGs are made out of material which was already metal-enriched prior to the current starburst in the progenitor galaxy: they were dynamically built from the outer disks of their parent galaxies where the metallicity is close to this value (Zaritsky et al. 1994). Gas with very low metal abundance exists in the outermost regions of spirals (Ferguson et al. 1998). However, the analysis carried out in Paper I shows that our TDG candidates are likely to contain a significant amount of old stars. Those had to be pulled out from regions in the parent stellar disk which were rather dense and could not be situated far beyond the optical radius. At their location, the metallicity should have been close to that measured in the tidal knots, i.e. $1 / 4$ to $1 / 3$ solar.

Some dynamical simulations (e.g. Barnes \& Hernquist 1998) show that the material of the inner and outer parts of a tidal tail came from smaller and larger radii of the progenitor disk, respectively. Due to the observed metallicity gradients of spiral disks, one might expect to see abundance gradients along tidal tails. In the systems AM 0537-292 and AM 1353-272, where we could observe several knots along the tails, however, we do not observe any systematic radial trend in their abundances. All the knots have nearly the same oxygen abundance around $12+\log (\mathrm{O} / \mathrm{H}) \approx 8.34$. This hints at a process which mixes material from different regions in the progenitor galaxy and has to be checked with new dynamical models.

The three objects with low metal abundances mentioned in Sect. 3.1 also seem to contain a strong old stellar component (Weilbacher et al. 2000). They are therefore most likely pre-existent companion galaxies, which are either projected onto the tidal features or falling into the interacting system.

\subsection{Velocity gradients}

What do the apparent velocity gradients tell us about the kinematics of the knots? Clearly such amplitudes are not normally observed in the H II regions of spirals where, after correction for the overall velocity field, only $15 \mathrm{~km} \mathrm{~s}^{-1}$ of residual vertical dispersion is observed (Jiménez-Vicente \& Battaner 2000). What is then the origin of such "gradients" in our objects? In fact, several different causes are possible. 


\section{Projection effects}

Most of the velocity gradients that we observe in the knots have an extent of 2 to $4^{\prime \prime}$, i.e. only a few times the FWHM of the typical seeing (see column 2 of Table 18). The lack of spatial resolution and related "smoothing" will cause physically disconnected regions with distinct velocities to appear as being part of a single kinematical entity. Were that the case, the observed velocity gradient would only be apparent and would hide projection effects. Some knots could in fact be external material seen in projection onto the tail. One may also have the cases where several knots of a bent tail or from even different tails project onto each other.

In Sect. 3.4 we noted three cases with two emission line regions within one apparent knot. Although we cannot confirm these instances as projection effects, these are candidates for this type of velocity gradient caused by projection.

\section{Streaming motions along the tails}

The overall velocity field of tidal tails is governed by streaming motions resulting from the gravitational tidal forces exerted during the collision. Identifying in them kinematically decoupled entities is difficult when, as in our sample, the global velocity field is only measured at discrete positions. An upper limit to the contribution from streaming motions can be obtained measuring the difference between the central velocity of the tidal knot and the systemic velocity of the parent galaxy. Generally, this velocity offset is much smaller than the small scale velocity gradient found in the tidal objects.

\section{Outflows associated with super-winds}

Outflows are known to accelerate gas to high velocities. Superwinds driven outflows seem to prevail in local starbursts (Heckman et al. 1990), in distant Lyman Break Galaxies (Pettini et al. 2001), and in dwarf galaxies (Martin 1998, Bomans et al. 2001). The kinematic signature of such winds are broad or double-peaked emission lines. They are due to the shell-like structures generated by stellar winds, which always show velocity components towards and away from the observer. If part of the shell was shielded from view by dust, it could also produce velocity gradients. We did not find any obvious broadening of the emission lines in regions characterized by a large velocity gradient and only a moderate extinction was measured there, which makes the dust obscuration of one component quite unlikely.

\section{Gravitationally dominated motion}

The last possible origin of the velocity gradients is gravitationally dominated motion within an entity that became kinematically independent from its host tail. Some of 
the $\Delta V_{\max }$ values measured are certainly too large to be caused by true Keplerian rotation of a virialized entity. If the knots were virially relaxed then one would derive a mass of $10^{10} M_{\odot}$ for an observed gradient of $\Delta V_{\max }=200 \mathrm{~km} \mathrm{~s}^{-1}$ over $1 \mathrm{kpc}$ from the virial theorem. In those cases it is possible that part of the velocity is due to motion of the $\mathrm{H}$ II regions towards the center of an entity in formation. The mass derived using the Virial Theorem would then strongly overestimate the real mass of the knot.

As the blue luminosity typically rises with mass, the velocity gradients are expected to increase with absolute magnitude, if they were all caused by the rotation of massive objects. This is not obvious in Fig. 33: we see high velocity gradients for both faint and bright knots. However, as most of the knots are still inside the tidal tail, show internal structure, and are not relaxed objects, we cannot assume Keplerian rotation. Also, all objects where we could measure velocity gradients are objects with strong emission lines, probably sites of strong star formation, where $M_{B}$ or in general optical luminosities are not a good measure for the mass of the knots. We will further pursue this question using near infrared luminosities in a future paper (Weilbacher et al., in prep.). We also see from Fig. 34 that the number of knots with visible gradients is higher in the outer regions $(20$ to $35 \mathrm{kpc})$ than in the inner regions $(<20 \mathrm{kpc})$ of our interacting systems. A reason for this could be that decoupled kinematics or bound knots are more difficult to produce in the denser parts of the inner tidal tails.

A good example of a self-gravitating object seems to be AM 1159-530a: the velocity gradient extends over 5." 5 or $1.6 \mathrm{kpc}$ with two bright $\mathrm{H}$ II regions at both ends. The gradient is confirmed by the detection of absorption lines between the H II regions with intermediate velocity. Another fainter $\mathrm{H}$ II region further westward has a discrepant velocity and seems to be projected onto this entity or falling into it.

\section{Limitations of these observations}

Clearly the large velocity gradients observed with EFOSC2 in a number of interacting systems should be confirmed with observations at higher spectral resolution. To unquestionably prove the amplitude of the gradients, velocity resolutions better than $200 \mathrm{~km} \mathrm{~s}^{-1}$ will be required. The system AM 1353-272 in our sample has actually been re-observed with FORS2 on the VLT at a higher spectral resolution confirming velocity gradients in several knots along both tidal tails of the interacting system, with velocity amplitudes in the range $24<\Delta V_{\max }<343 \mathrm{~km} \mathrm{~s}^{-1}$ (Weilbacher et al. 2002). To overcome the poor sampling of the gradients, imaging with higher spatial resolution is also needed. $\mathrm{H} \alpha$ observations with the Fabry-Perot technique (see e.g. Mendes de Oliveira et al. 2001) in some interacting systems are in progress (Duc et al. 2002, in prep.). 


\subsection{Selecting Tidal Dwarf Galaxies}

Are any of the knots present in the interacting systems studied here the progenitors of TDGs? We describe below the steps required to identify a genuine TDG according to the definition cited in Sect. 1 and check whether the objects in our sample fulfill them. A necessary first step is to select the brightest knots in the tidal tails with luminosities of dwarf galaxies $\left(-10.5 \lesssim M_{B} \lesssim-17.0 \mathrm{mag}\right.$, from Paper I). Together with the new objects presented in Table 19 we start with 44 TDG candidates (including AM 0529-565D).

\section{Redshift criterion}

The selection method from Paper I to disentangle star-forming knots along tidal features from background sources using images in three optical broad band filters in combination with evolutionary synthesis modeling is an efficient method (Sect. 2.2). Nevertheless, it is important to check the association with the main interacting system by measuring the spectroscopic redshift of the candidates. This leaves 29 candidates.

\section{Metallicity criterion}

Low-mass companion galaxies, existing prior to the collision, can be discriminated from TDG candidates from their low metallicities. While the tidal knots in our sample have almost constant metallicities around $12+\log (\mathrm{O} / \mathrm{H}) \approx 8.3$, three objects close to the tidal features have abundances at least 0.4 dex lower than the tidal knot with the lowest abundance. This is puts them close to the luminosity-metallicity relationship of normal dwarf galaxies. A tidal origin can therefore be excluded for these three exceptions. 26 candidates for TDGs are left.

\section{Dynamical criterion}

Genuine TDGs should be gravitationally bound. We do not find classical rotation curves or typical velocity dispersion in any of our TDG candidates. Instead, we find several cases of apparent velocity gradients in the knots in the tidal tails. While we cannot yet exclude all other possible causes 13 TDG candidates seem to have kinematics decoupled from the hosting tail. Further data with high resolution will be necessary to prove these gradients as internal kinematics of the individual candidates. 


\section{Final sample of TDG candidates}

The 13 objects identified as good TDG candidates represent $46 \%$ of the knots that were observed spectroscopically and half of the knots left after selecting the knots depending on their metallicity. Note that - given the low resolution of our spectroscopic data - they cannot yet be considered as real TDGs.

From our 14 interacting galaxies 5 (i.e. about 1/3 of the sample systems) have TDG candidates with kinematical signatures. Of those systems, one (AM 1054-325) has only one TDG candidate, which has an optical appearance very similar to other knots in the same system for which we did not detect decoupled kinematics. The other system with only one TDG, AM 1159-530, has one tail with relatively high and one with very low surface brightness. The massive TDG is positioned at the end of the bright tail. In both galaxies with two TDGs (AM 0537-292 and AM 0547272), they are positioned in the tails on opposite sides of the nucleus. The system AM 1353-272 contains more that half of the TDG candidates we have finally selected, distributed over both tails.

\section{Conclusions}

We have analyzed a sample of 14 southern interacting galaxies using deep, low resolution spectrophotometry. The knots in the tidal features were previously identified as good TDG candidates by comparing optical broad band imaging with evolutionary synthesis models. The spectra were first used to investigate the association with the interacting system by comparing the redshifts. All knots that were detected are actually associated with the parent galaxies. This shows that our photometric method is a powerful tool for preselecting TDGs. The few knots which were not detected in our spectra could be of a different nature.

We determined the oxygen abundances of the knots and found a mean value of $12+$ $\log (\mathrm{O} / \mathrm{H})=8.34$ with a scatter of \pm 0.2 dex. Both the mean value and the scatter are in good agreement with those found in the outer disks of spiral galaxies, which precludes the possibility that these knots could be pre-existing objects infalling onto the interacting systems from outside. Three dwarf galaxies close to the tidal features are unlikely to be TDGs due to their low metallicity.

As predicted by our photometric models, the knots are actively forming stars, and some knots may even be starbursts with high $\mathrm{H} \alpha$ luminosities. $L(\mathrm{H} \alpha)$ of the knots within our spectroscopic slits is higher by two orders of magnitude than that of typical individual H II regions in spiral disks and lower than that of local star-forming galaxies by a factor of 10 . However, the flux represents many individual $\mathrm{H}$ II regions whose number depends on the slit width and the distance of the interacting system. Due to the overlap of the distribution of $L(\mathrm{H} \alpha)$ with "normal" giant $\mathrm{H}$ II regions in 


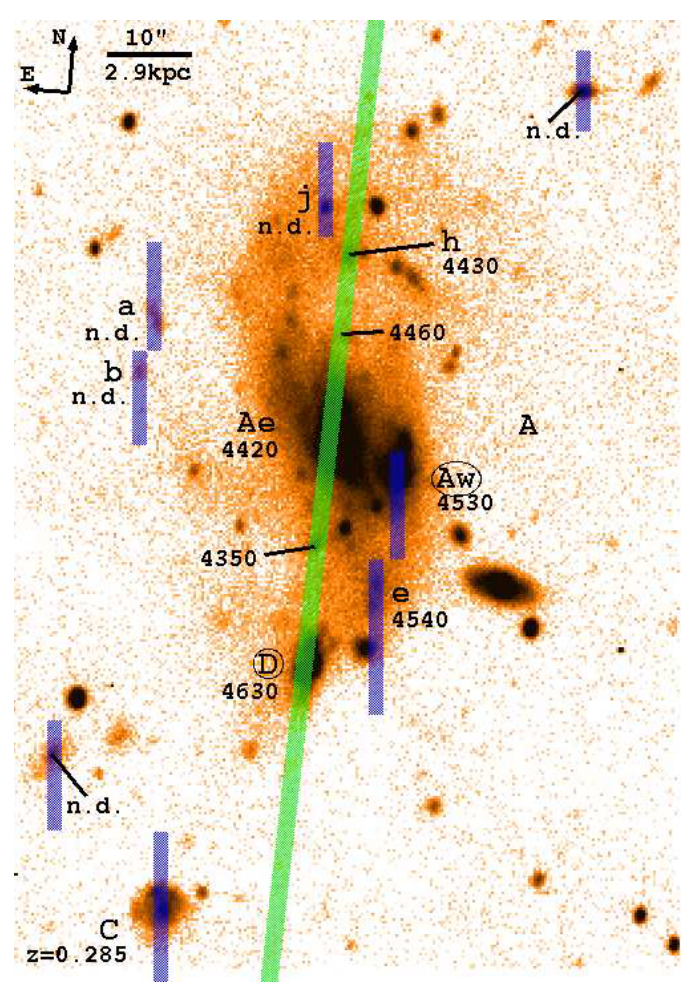

Figure 36: Slit positions in the field of AM 0529-565. Relevant objects are marked along with their central velocities. The labels of objects with low metallicity are circled.

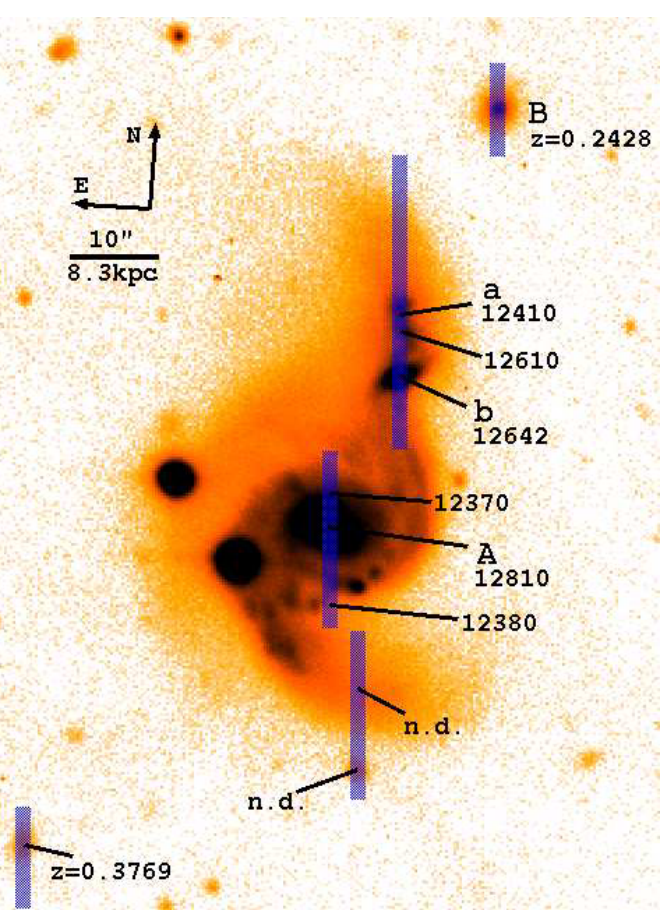

Figure 37: Slit positions in the field of AM 0607-444. Relevant objects are marked along with their central velocities.

spiral disks and the difference to dwarf galaxy luminosities, $L(\mathrm{H} \alpha)$ is not a good criterion to discriminate TDGs from other phenomena.

We see surprisingly strong velocity differences in some interacting galaxies, and 13 TDG candidates show kinematic signatures which could be internal velocity gradients in these knots. The velocity amplitudes are in some cases more than one order of magnitude higher than those observed in spiral disks. Our analysis and interpretation is hampered by the small angular size of these gradients, which are barely resolved in $\sim 1^{\prime \prime}$ seeing, so we could not discriminate possible decoupled kinematics from other phenomena like chance alignment of multiple bodies which mimic velocity gradients. We can only prove the existence of one strong velocity gradient within the TDG AM 1159-530a, where we see the gradient extending over $5 . " 5$, which is likely due to the gravitational forces within the body of the TDG. To confirm the velocity gradients of the knots in the tidal tails as decoupled kinematics governed by internal gravitation an improvement of at least a factor two in spatial 


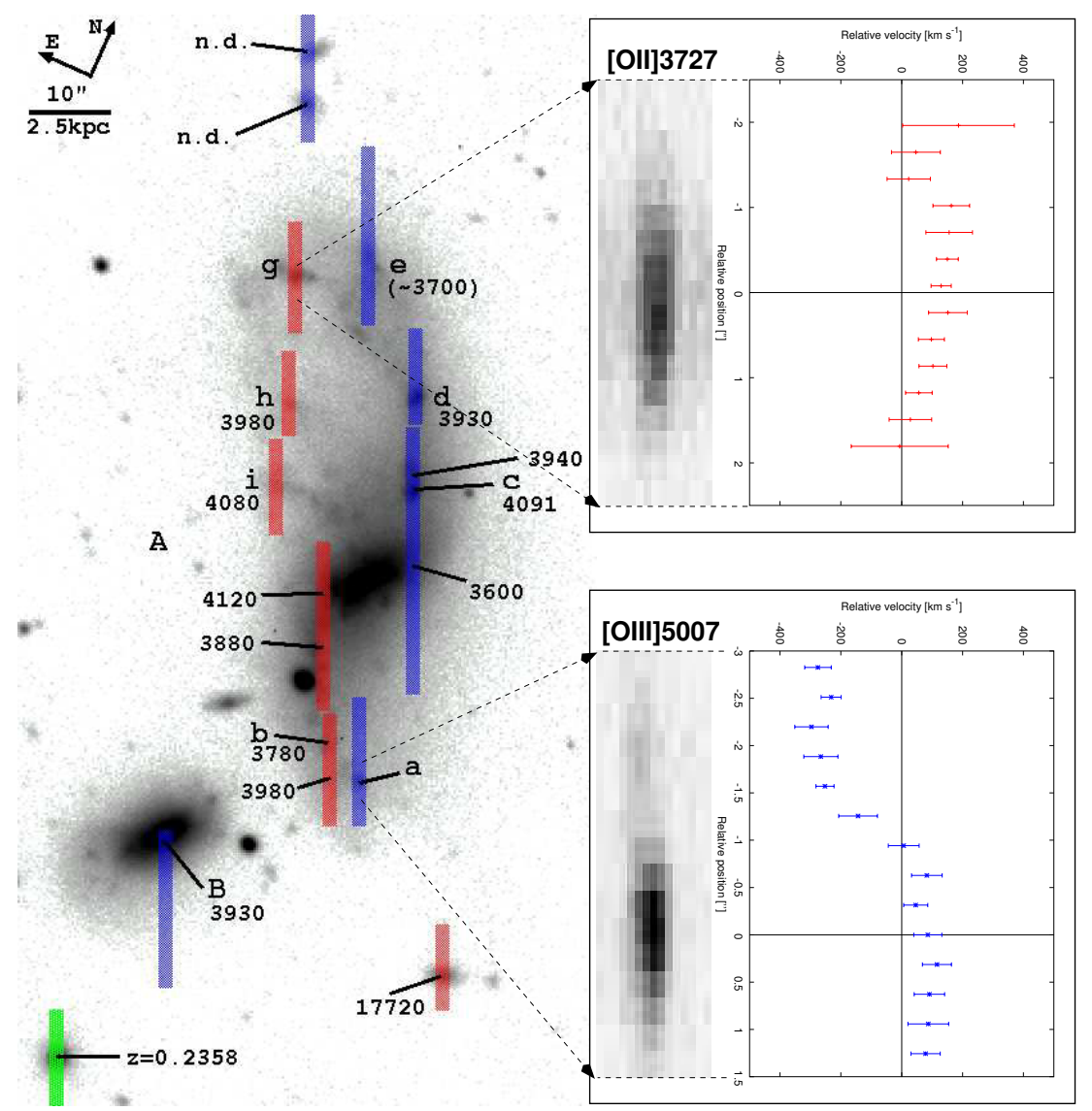

Figure 38: Slit positions in the field of AM 0537-292. Relevant objects are marked along with their central velocities. Velocity gradients in AM 0537-292a (from profile fits for $\mathrm{H} \alpha,[\mathrm{O} \mathrm{III}] 5007$ with errors $<0.25 \%$ ) and in AM 0537-292g (from profile fits for $\mathrm{H} \alpha,[\mathrm{O} I I] 3727$ with errors $<0.5 \%$ ) are shown together with their $2 \mathrm{D}$ spectra for [O III]5007 and [O II] 3727, respectively. The velocity zeropoint for both plots is $3900 \mathrm{~km} \mathrm{~s}^{-1}$. The slit on the background galaxy to the lower left was observed with both MOS masks, both giving consistent redshift.

resolution is required. Higher velocity resolution (better than $200 \mathrm{~km} \mathrm{~s}^{-1}$ ) would be desirable to confirm the amplitude of the velocity gradients. The discussion of both emission line luminosities and velocity gradients is restricted by the nature of our multi-slit spectroscopic data, which allows us to analyze only discrete parts of the whole interacting system. Narrow band imaging would be required to confirm and interpret the full range of $\mathrm{H} \alpha$ luminosities found in the TDG candidates. Furthermore, observational methods like Fabry-Perot- or integral field spectroscopy would be required to fully analyze the velocity distributions of the ionized gas in the interacting galaxies and the TDG candidates. 


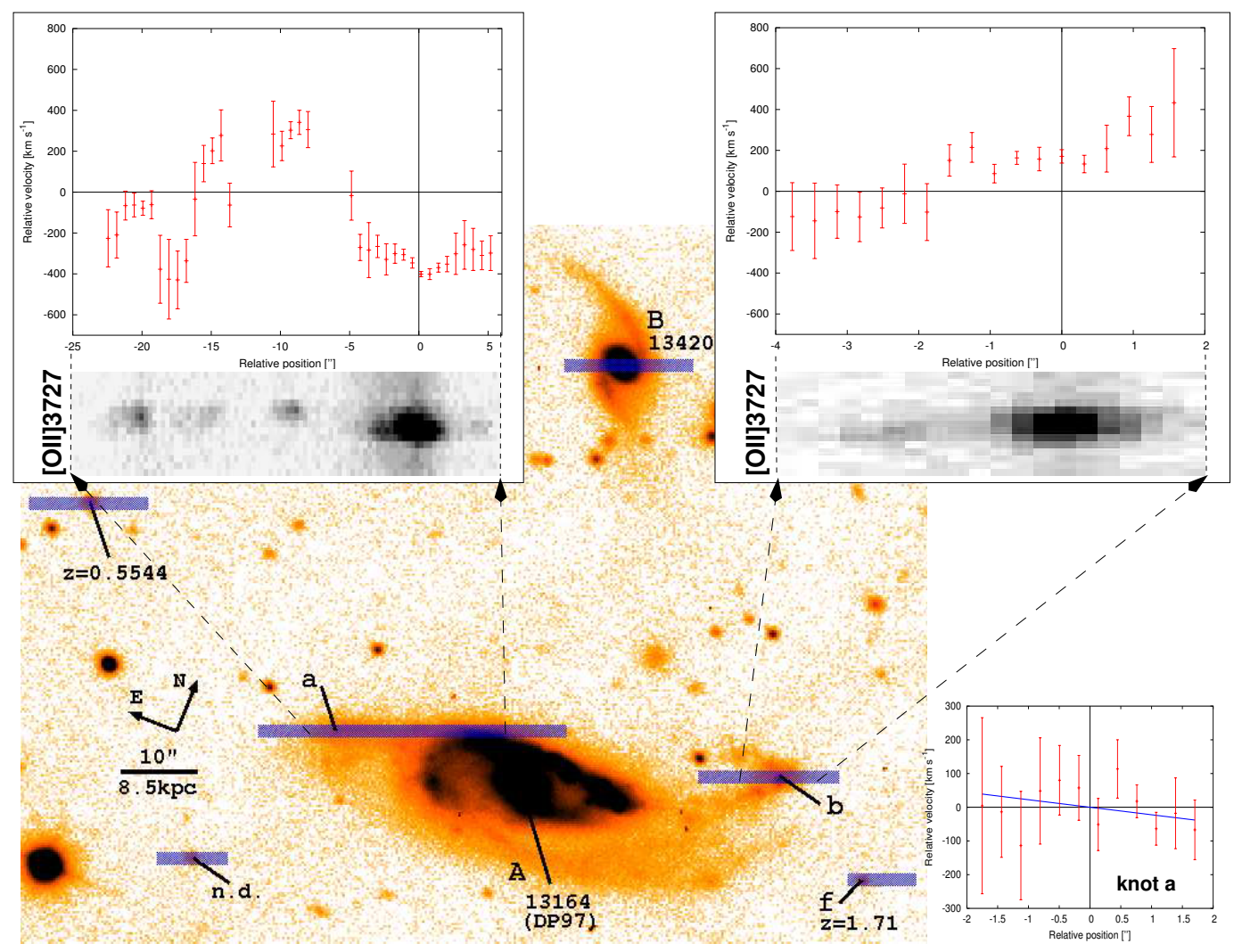

Figure 39: Slit positions in the field of AM 0547-244. Relevant objects are marked along with their central velocities. Velocity gradients (from profile fits for $\mathrm{H} \alpha$, [O II] 3727 with errors $<0.25 \%$ ) in the eastern tail of AM 0547-244 including ' $a$ ' (binned by 2 pixels) and in AM 0547-244b are shown together with their 2D spectra for [O II] 3727. The velocity zeropoint for both plots is $13200 \mathrm{~km} \mathrm{~s}^{-1}$. We also show the velocity field of knot 'a' after subtraction of the tidal streaming motion and its linear fit in the lower right corner.

Finally, the data collected in this paper will help us refine our spectrophotometric evolutionary synthesis models for analyzing the starburst properties of the TDG candidates and to derive more accurate predictions of their future evolution.

We would like to thank L.-M. Cairós for careful reading and helpful comments on the manuscript, and R. Hessman who was very helpful in improving the English. Many thanks to Pierre Leisy for exceptionally dedicated and helpful support during the observing run in January 2000 on La Silla. PMW acknowledges partial support from DFG grants FR 916/6-1 and FR 916/6-2. 


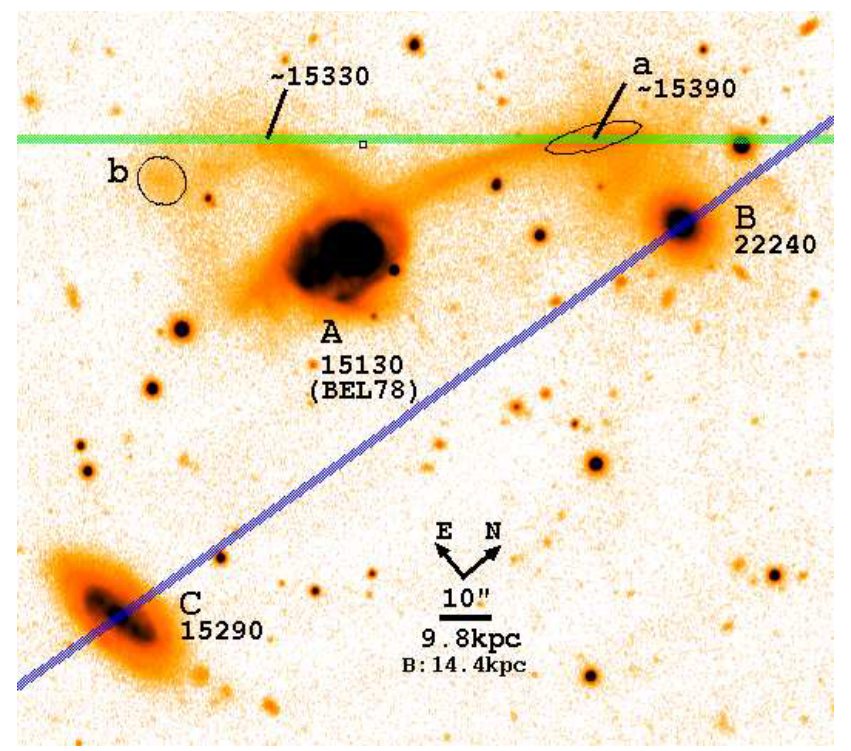

Figure 40: Slit positions in the field of AM 0547-474. Relevant objects are marked along with their central velocities.

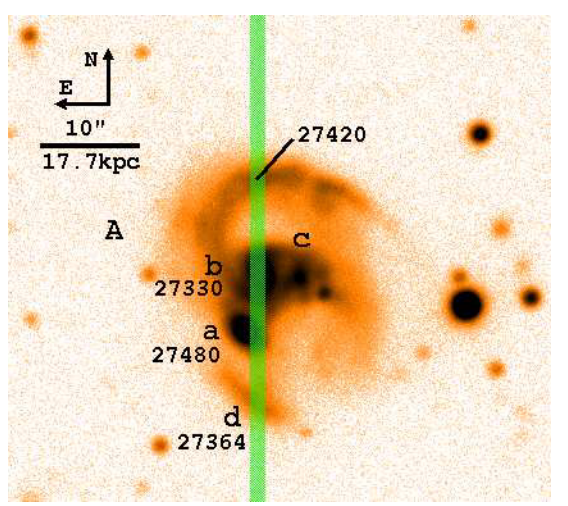

Figure 41: Slit position in the field of AM0642645. Relevant objects are marked along with their central velocities. Here we used the NTT-SUSI $R$-band image of Weilbacher et al. (2000).

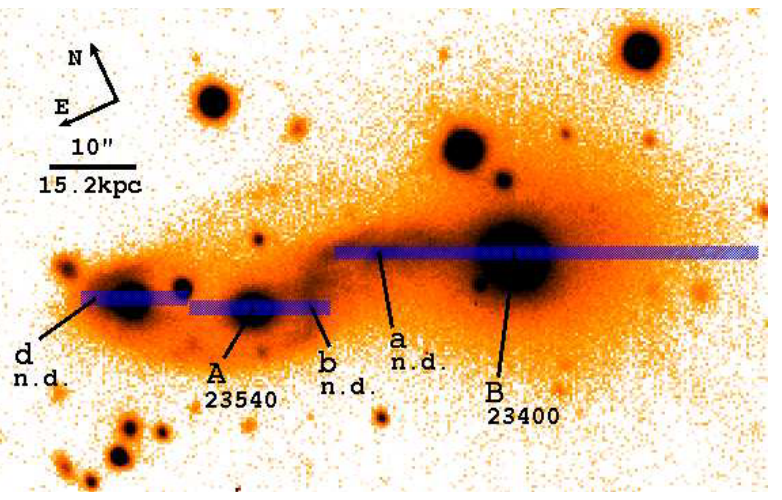

Figure 42: Slit positions in the field of AM 0748-665. Relevant objects are marked along with their central velocities. None of the TDG candidates from Paper I has a firm detection. 


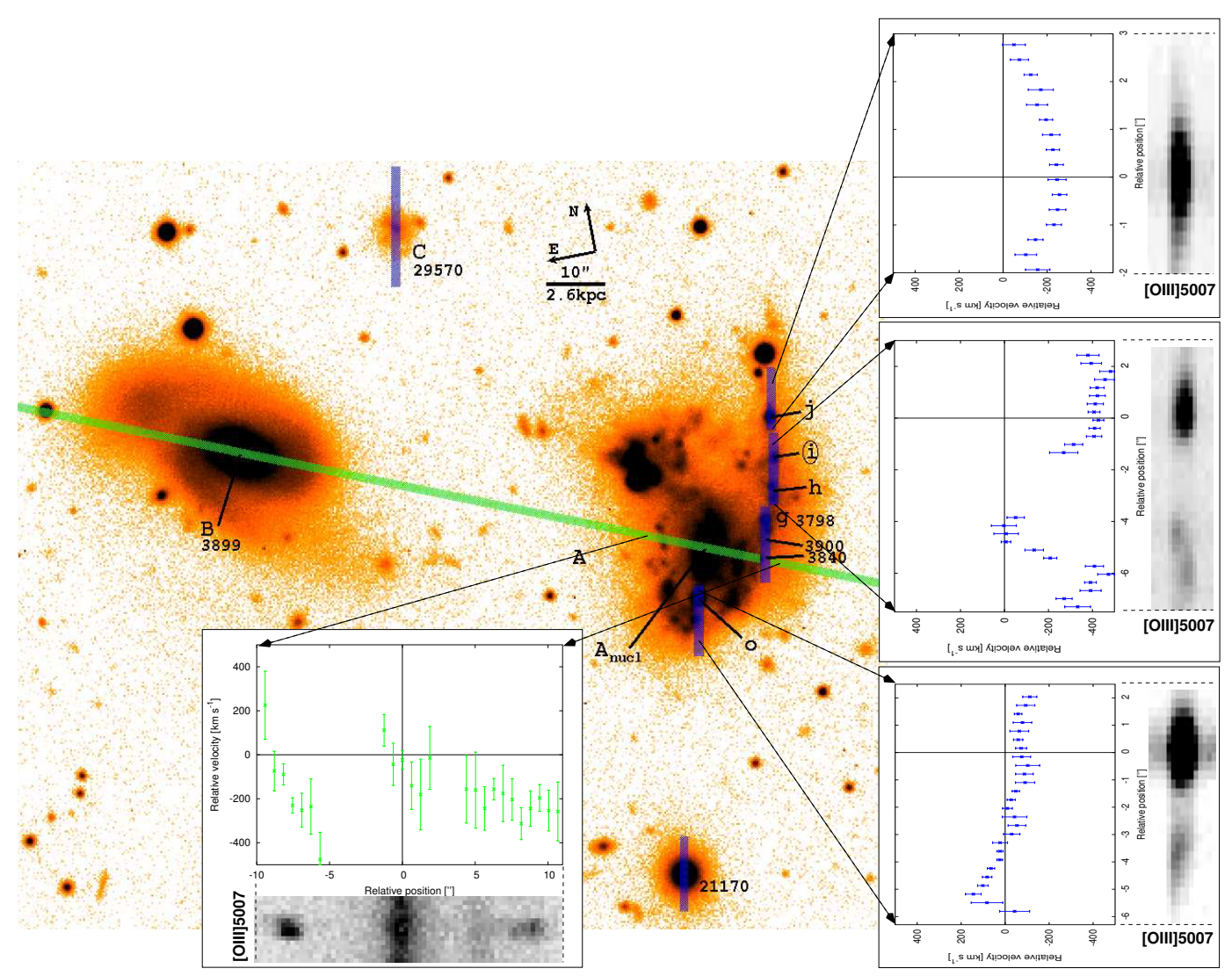

Figure 43: Slit positions in the field of AM 1054-325. Relevant objects are marked along with their central velocities. Velocity gradients through the central region of AM 1054-325A (from profile fits of our long-slit of $\mathrm{H} \alpha,[\mathrm{O}$ III]5007,[O II] 3727

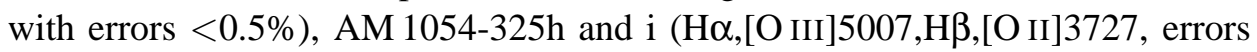
$<0.25 \%$ ), AM 1054-325j ( $\mathrm{H} \alpha,[\mathrm{O} \mathrm{III}] 5007, \mathrm{H} \beta$, errors $<0.25 \%$, and AM 1054$325 \mathrm{o}(\mathrm{H} \alpha,[\mathrm{O} \mathrm{III}] 5007, \mathrm{H} \beta$, errors $<0.25 \%)$ are shown together with their $2 \mathrm{D}$ spectra for [O III] 5007. The velocity zeropoint for all plots is $3970 \mathrm{~km} \mathrm{~s}^{-1}$.

\section{A Photometric results of the 4 new systems}

Here we briefly discuss the photometric results of $B, V, R$ imaging of the four new interacting system in our sample. The analysis was done using individual polygonal photometric apertures fitted to surface brightness contours for each object, as described in detail in Paper I. 


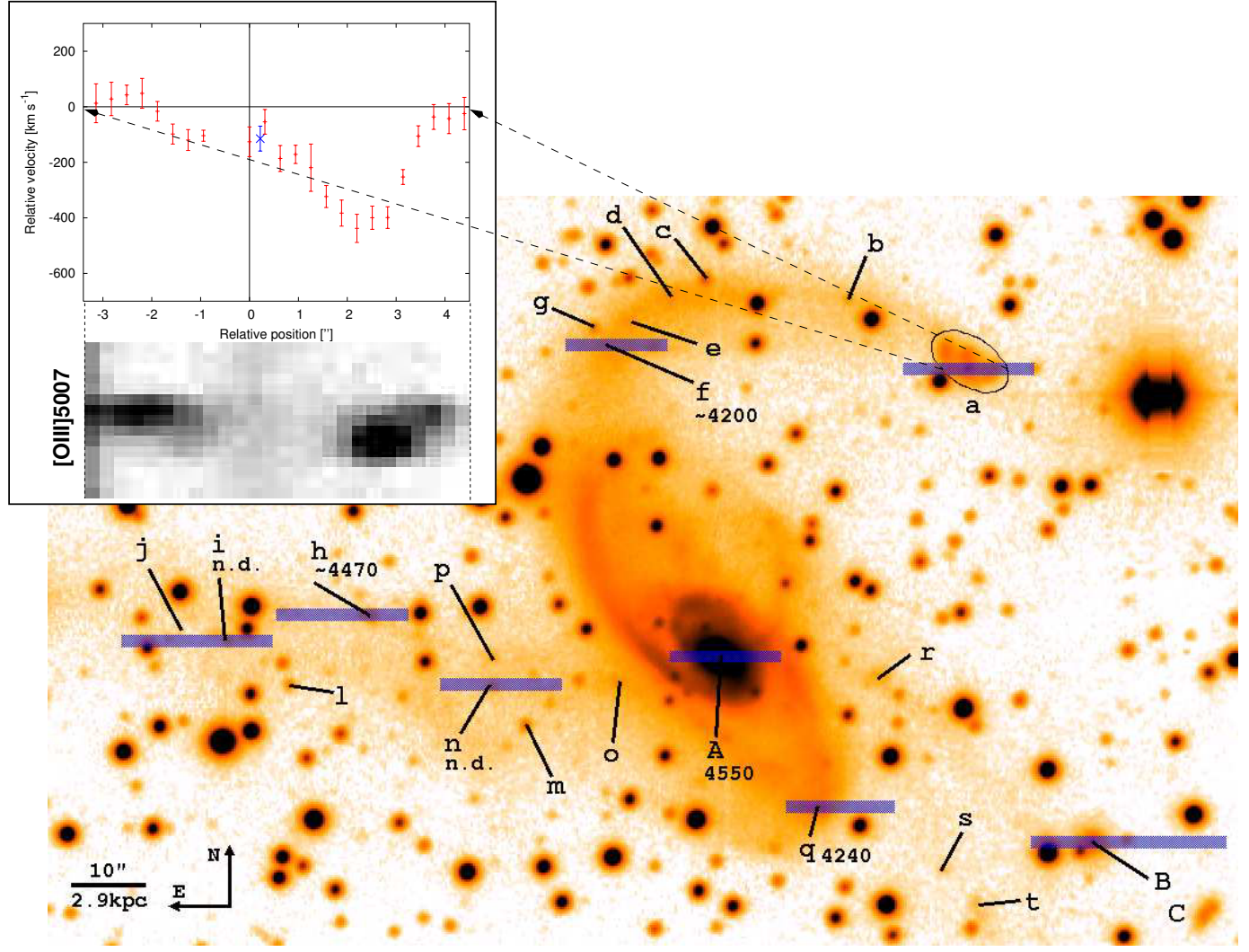

Figure 44: Slit positions in the field of AM 1159-530. Relevant objects are marked along with their central velocities. A systemic velocity of $4550 \mathrm{~km} \mathrm{~s}^{-1}$ is taken as zeropoint for the velocity curve derived from fitting $\mathrm{H} \alpha$, [O III] $] 5007$, and $\mathrm{H} \beta$ (with errors $<0.25 \%$ ). The $2 \mathrm{D}$ spectrum displays the line [O III] 5007 . The larger cross denotes one datapoint derived from fits to higher order Balmer lines.

\section{A.1 AM 0547-474}

This system (Fig. 40, also known as ESO 205-IG 003) consists of what on the first glance would seem to be three galaxies: 'A', a 'merger' with two tidal tails; 'B', an elliptical near the end of the northern tail; and ' $C$ ', an early type galaxy without obvious connection to the other objects.

The ends of both tidal tails show weak concentrations, labeled ' $a$ ' and ' $b$ '. Both have integrated absolute magnitudes of dwarf galaxies, $M_{B, \mathrm{a}}=-17.5 \mathrm{mag}$ and $M_{B, \mathrm{~b}}=$ $-14.8 \mathrm{mag}$, taken at $V$ contours of 24.5 and $25.5 \mathrm{mag} / \square^{\prime \prime}$, respectively. But as the material in ' $a$ ' and ' $b$ ' is not strongly concentrated and there is no starburst (colors $B-V=0.60 \ldots 0.77 \mathrm{mag}$ ), it is unlikely that they are TDGs, although it is possible that at a later stage in the evolution of the system the concentrations may increase and form real TDGs. 


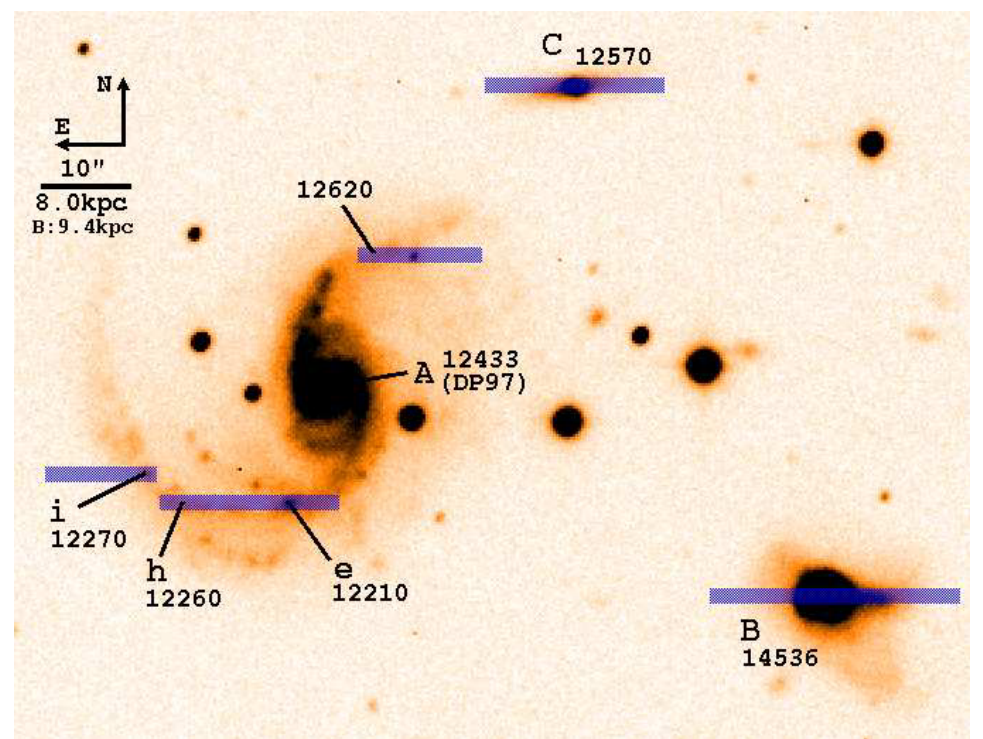

Figure 45: Slit positions in the field of AM 1208-273. Relevant objects are marked along with their central velocities.

\section{A.2 AM 1159-530}

'A' (Fig. 44, also cataloged as ESO 171-IG 005) is classified as SAB(rs)a:pec. It clearly shows strong signs of interaction and has two long tidal tails, which contain several faint knots. Although no interacting counterpart is visible on our frames, Corwin et al. (1985) mention a possible companion, which is an uncataloged ob-

Table 19: New TDG candidates from photometry

\begin{tabular}{l|ccc|cc} 
Designation & $\begin{array}{c}M_{B} \\
{[\mathrm{mag}]}\end{array}$ & $\begin{array}{c}B-V \\
{[\mathrm{mag}]}\end{array}$ & $\begin{array}{c}\text { Best fit } \\
\text { Model ID }^{1}\end{array}$ & $\begin{array}{c}\text { burst age }^{2} \\
{[\mathrm{Myr}]}\end{array}$ & $\begin{array}{c}\text { burst strength }^{2} \\
b\end{array}$ \\
\hline AM 1159-530a & -16.26 & 0.13 & $6\left(Z_{3}\right)$ & 4 & 0.18 \\
AM 1159-530h & -13.57 & 0.38 & $5\left(Z_{1}\right)$ & $\sim 1$ & 0.09 \\
\hline AM 1237-364g & -15.46 & 0.18 & $6\left(Z_{1}\right)$ & 6 & 0.18 \\
\hline AM 1324-431c & -15.02 & 0.21 & $6\left(Z_{3}\right)$ & 3 & 0.18 \\
AM 1324-431d & -14.31 & 0.37 & $2\left(Z_{3}\right)$ & $<1$ & 0.05 \\
AM 1324-431e & -13.77 & 0.25 & $2\left(Z_{1}\right)$ & 5 & 0.05 \\
AM 1324-431h & -14.10 & 0.25 & $5\left(Z_{1}\right)$ & 4 & 0.09
\end{tabular}

${ }^{1}$ from Table 2 of Paper I.

${ }^{2}$ using the best fit model from Paper I. 


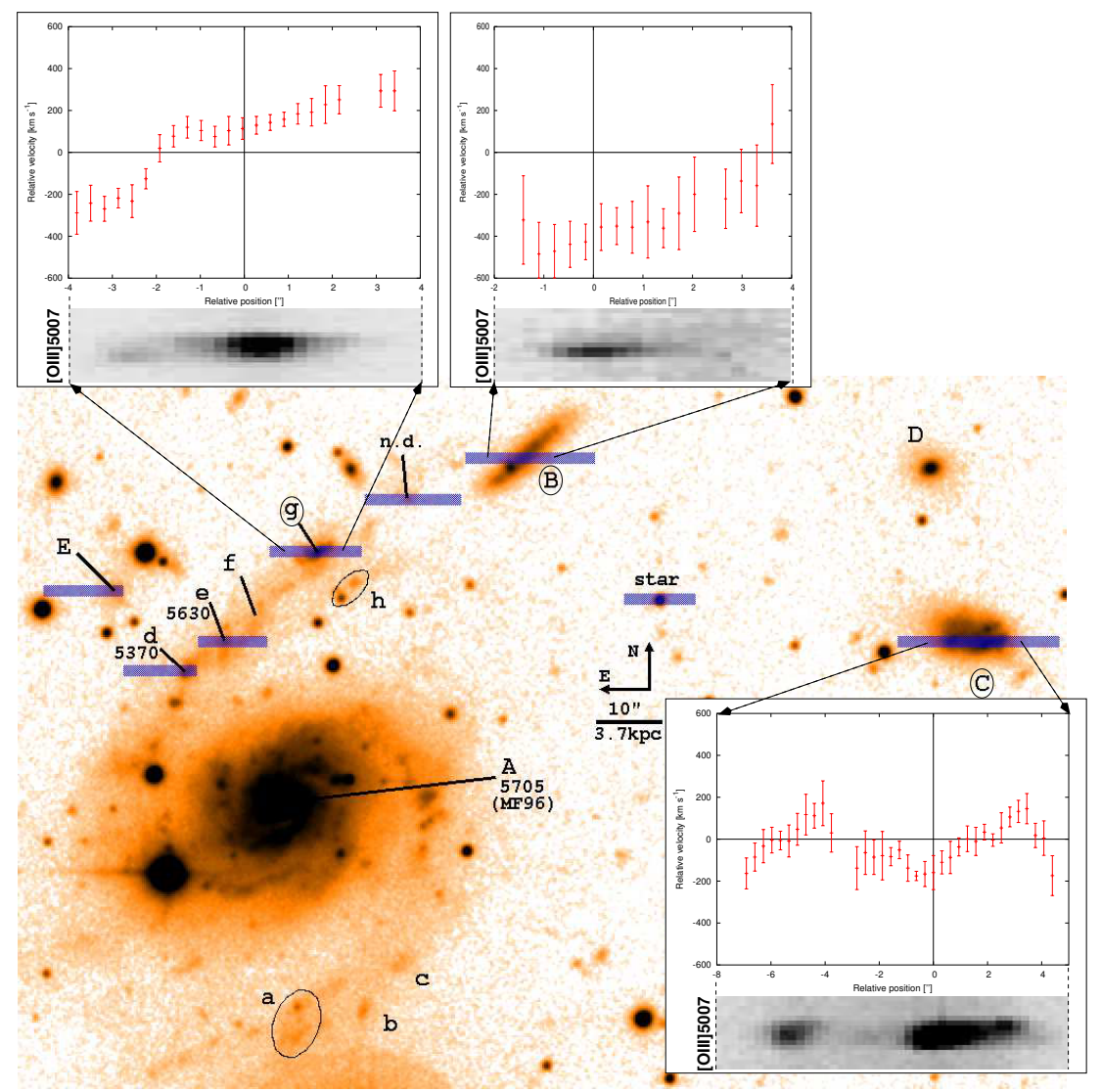

Figure 46: Slit positions in the field of AM 1237-364. Relevant objects are marked along with their central velocities. Velocity gradients of the companion galaxies AM 1237-364B (profile fits of [OIII5007],H $\beta,[\mathrm{O} \mathrm{II}] 3727$ with errors $<0.5 \%$ ), AM 1237-364C (Ho,[OIII5007],H $\beta,[\mathrm{O} \mathrm{II}] 3727$, errors $<0.25 \%)$, and the knot AM 1237-364g $(\mathrm{H} \alpha,[\mathrm{OIII} 5007], \mathrm{H} \beta, \mathrm{H} \gamma$, errors $<0.25 \%)$ are shown together with their 2D spectra for [O III]5007. The velocity zeropoint for all plots is $5705 \mathrm{~km} \mathrm{~s}^{-1}$.

ject of comparable magnitude $5^{\prime}$ to the SSW at RA=12:01:15.8, DEC $=-53: 25: 22$ (J2000).

The tails contain several faint knots. At the end of the northern tail a massive knot is apparent, labeled 'a' $\left(M_{B}=-16.3 \mathrm{mag}\right)$, which - from its colors - is a good candidate for a TDG. There are other fainter knots in the tails, some of which could be built from tidal material, but only the colors and luminosity of knot ' $h$ ' match our selection criteria for TDG candidates.

Results for this system have already been published in Weilbacher \& Duc (2001) and Weilbacher et al. (2001). 


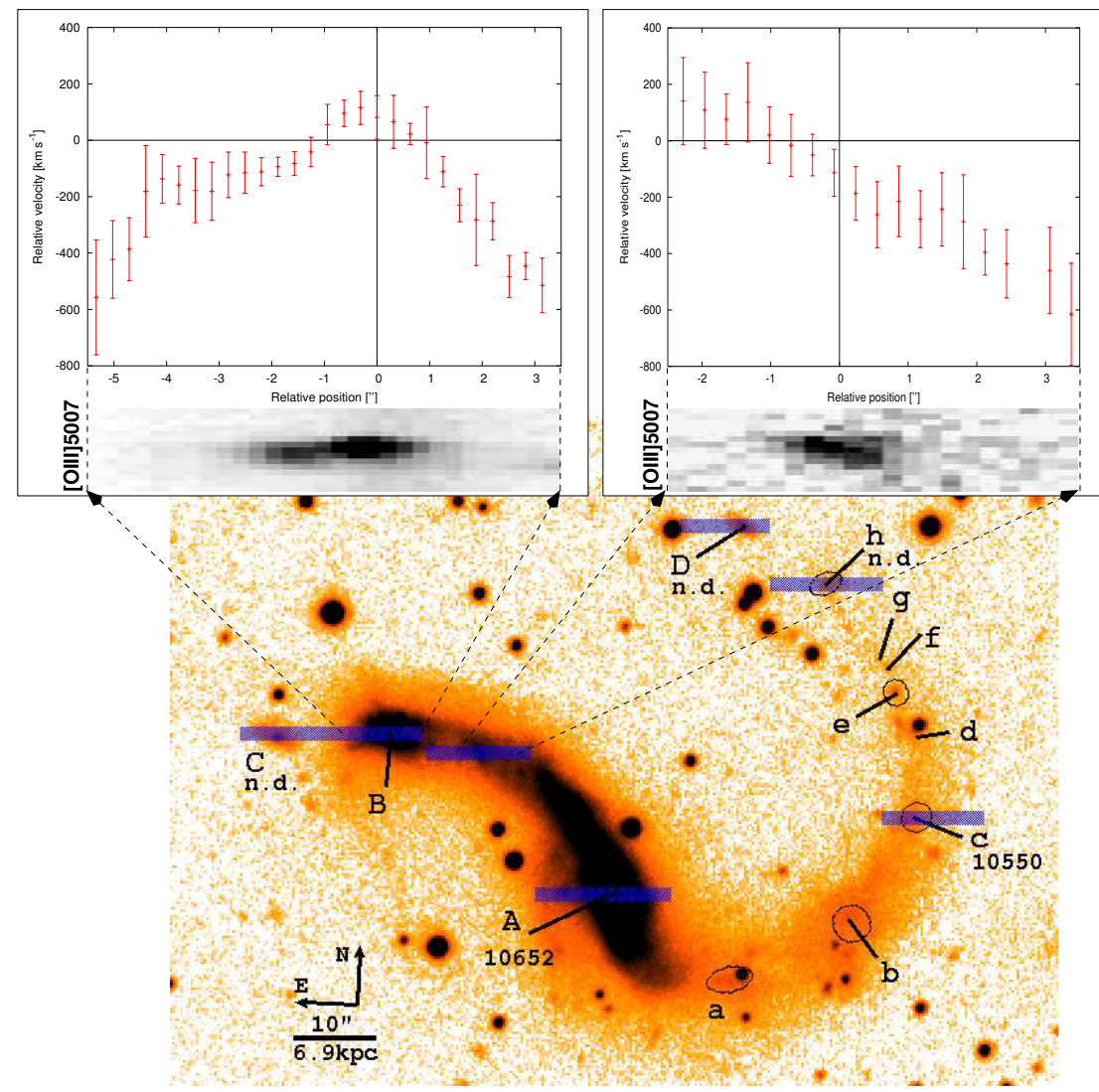

Figure 47: Slit positions in the field of AM 1324-431. Relevant objects are marked along with their central velocities. Velocity gradients between ' $\mathrm{A}$ ' and ' $\mathrm{B}$ ' and in AM 1324-431B (from profile fits of $\mathrm{H \alpha},[\mathrm{O}$ III]5007, $\mathrm{H} \beta,[\mathrm{O}$ II $] 3727$ with errors $<0.25 \%$ ) are shown together with their $2 \mathrm{D}$ spectra for $[\mathrm{O} \mathrm{III}] 5007$. The velocity zeropoint for the plots is $10680 \mathrm{~km} \mathrm{~s}^{-1}$.

\section{A.3 AM 1237-364}

The spiral galaxy 'A' (Fig. 46, also designated as ESO 381-G 005) has the largely undisturbed appearance of an Sbc. The velocity was measured by Mathewson \& Ford (1996) as $V_{\mathrm{A}}=5705 \mathrm{~km} \mathrm{~s}^{-1}$. Two nearby galaxies 'B' and 'C' both have blue colors compatible with a nearby starburst, and are therefore probable companions of 'A'. 'B' is very near the northern tail, but has a very regular appearance, and is therefore very unlikely to be a TDG.

Several knots in the arms are apparent, which have absolute magnitudes of dwarf galaxies ('a' to 'f', $M_{B}=-12.1 \cdots-15.2 \mathrm{mag}$ ). Most of them are very diffuse, and probably conglomerates of H II regions generally found in spiral arms, which the seeing causes to appear as single surface brightness peaks. Only the massive knot 


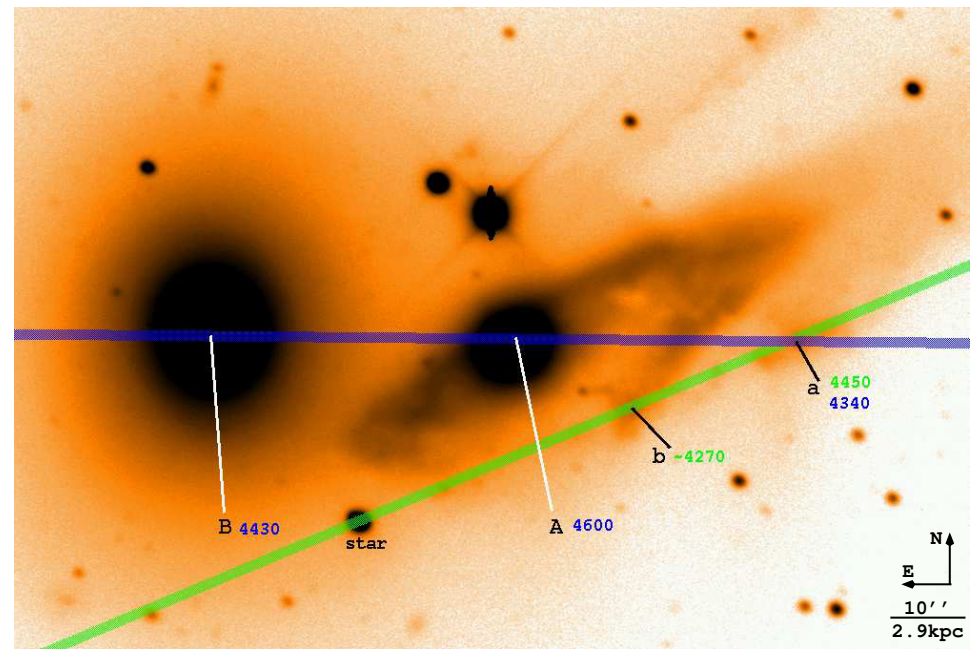

Figure 48: Slit positions in the field of AM 1325-292. Relevant objects are marked along with their central velocities. Here we used the NTT-SUSI $R$-band image of Weilbacher et al. (2000).

' $\mathrm{g}$ ' at the end of the northern arm is dense enough and far away from the nucleus. Its colors fit well with one of our starburst models from Paper I, so we select it as a TDG candidate.

\section{A.4 AM 1324-431}

This system (Fig. 47, also known as ESO 270-G 012) with the interacting galaxies ' $\mathrm{A}$ ' and ' $\mathrm{B}$ ' shows a bright bridge between both nuclei and a long tail to the northwest. The tail hosts several knots ('a' to ' $h$ '), all of which have blue colors in the range of local starburst knots, and are probably sources physically associated with the tail. We select the brighter knots, far away from the nucleus of ' $A$ ' and in less dense regions of the tail as TDG candidates: 'c', 'd', 'e', and ' $h$ '. They are very well matched by the colors of the photometric models in Paper I. Two other objects ' $C$ ' and 'D' have red colors with $B-V>1.0 \mathrm{mag}$ and are very likely background sources.

\section{B Notes about individual systems}

\section{B.1 AM 0529-565}

(Fig. 36) There seem to be three velocity groups involved in this interaction, 'Ae' and the "disk" have $V_{\mathrm{A}} \sim 4420 \mathrm{~km} \mathrm{~s}^{-1}, V_{\mathrm{Aw}} \sim 4530 \mathrm{~km} \mathrm{~s}^{-1}$ and $V_{\mathrm{D}} \approx 4630 \mathrm{~km} \mathrm{~s}^{-1}$. 
Both 'Aw' and ' $\mathrm{D}$ ' have lower oxygen abundances of $7.8 \pm 0.1$ and $7.6 \pm 0.2$, respectively, than the knots in the disk (around 8.3, Weilbacher et al. 2000). Together with the more central location of 'Ae' with regard to the disk, this suggests that both 'Aw' and 'D' are pre-existing dwarf galaxies which are infalling towards or interacting with ' $\mathrm{A}$ ', whose nucleus is 'Ae'. Both dwarf galaxies experience strong starbursts due to the interaction.

\section{B.2 AM 0537-292}

(Fig. 38) 'A' and 'B' are indeed interacting with a central velocity difference of $\sim 70 \mathrm{~km} \mathrm{~s}^{-1}$. 'A' shows strong internal velocity differences, e.g. $520 \mathrm{~km} \mathrm{~s}^{-1}$ between the ends of the bar, $490 \mathrm{~km} \mathrm{~s}^{-1}$ between northern end of the bar and the surface brightness peak of ' $c$ '. Given the strong velocity differences within ' $a$ ' and 'c', we suggest that there could be some projection effect involved which mimics higher velocity differences between the knots and lower surface brightness of the tidal tails.

\section{B.3 AM 0547-244}

(Fig. 39) We confirm here that ' $\mathrm{B}$ ' actually is the interacting companion of 'A', they have a relative velocity $\sim+250 \mathrm{~km} \mathrm{~s}^{-1}$ (taking the value of Donzelli \& Pastoriza 1997, for the nucleus of 'A'). If our velocities are not offset from the value of Donzelli \& Pastoriza (1997) then 'A' exhibits strong internal velocity differences, about $400 \mathrm{~km} \mathrm{~s}^{-1}$ from the nucleus to the emission-line region in the northern part of the ring.

Despite the low metallicity required to successfully model the broad-band colors in Paper I, we derive oxygen abundances of about $1 / 3$ solar for the regions east of the nucleus and ' $b$ ', but the colors of those would also be roughly consistent with the higher metallicity model in Paper I. The TDG candidate ' $a$ ', however, does have a lower oxygen abundance of about 1/5 solar.

As already indicated by its the colors, ' $\mathrm{f}$ ' is confirmed as a background object. The spectrum shows several broad emission lines matching $z=1.71$ so that we can classify it as an AGN.

\section{B.4 AM 0547-474}

(Fig. 40) As already noted by West et al. (1981) 'B' has a different velocity from the others, $V_{\mathrm{A}}=15130 \pm 40 \mathrm{~km} \mathrm{~s}^{-1}$ (Bergwall et al. 1978) and $V_{\mathrm{C}}=15330 \pm$ $100 \mathrm{~km} \mathrm{~s}^{-1}$. 'B' actually is a background galaxy with $V_{\mathrm{B}}=22240 \pm 110 \mathrm{~km} \mathrm{~s}^{-1}$. Both ' $\mathrm{B}$ ' and ' $\mathrm{C}$ ' show absorption line spectra, while according to Bergwall et al. (1978) 'A' shows strong emission lines. 
The tips of the tails, designated ' $a$ ' for the northern tail and ' $b$ ' for the southern tail, do not show emission lines despite the moderately blue colors. From very faint absorption lines we could derive rough estimates of the velocities, $V_{\mathrm{a}}=15390 \pm$ $100 \mathrm{~km} \mathrm{~s}^{-1}$ and $V_{\mathrm{b}}=15330 \pm 80 \mathrm{~km} \mathrm{~s}^{-1}$.

\section{B.5 AM 0607-444}

(Fig. 37) Massive extinction in ' $\mathrm{b}$ ' of $\left(A_{V} \approx 3.3 \mathrm{mag}\right)$ explains the very red colors which led us to speculate in Paper I that it could be a background object. The emission line region 2 "' 6 north of the center of ' $b$ ', however, suffers from much lower extinction $\left(A_{V} \approx 0.8 \mathrm{mag}\right)$. The light from the much bluer region ' $\mathrm{a}$ ' is probably also strongly reddened, as the $\mathrm{H} \alpha$ emission is quite strong and an $\mathrm{H} \beta$ line could not be detected in the continuum.

Given our new data on this system (the distance was not known before), it seems now likely that ' $b$ ' represents the (remaining) nucleus of the interacting partner of 'A', which was possibly stripped of its disk material by the interaction. Both are interacting with a velocity difference of $200 \mathrm{~km} \mathrm{~s}^{-1}$.

Within 'a' a weak velocity gradient can be seen, but it is too weak to be significant given our low spectral resolution, and could also reflect the overall motion along the northern tail, in particular since the slit is oriented in the same direction.

\section{B.6 AM 0642-645}

(Fig. 41) This galaxy seems to be a merger of two or three galaxies, the nuclei of which are still separated and were designated ' $a$ ', 'b', and 'c' in Paper I. The long-slit was positioned to cover the nuclei 'a', 'b', and the southern plume ' $d$ '. The velocities we measured place this object at $V_{\mathrm{A}}=27410 \pm 130 \mathrm{~km} \mathrm{~s}^{-1}$ or at a distance of $365 \mathrm{Mpc}$, which makes it the most distant system in our sample. The system then has a north-south diameter of $48.7 \mathrm{kpc}$ and the nuclei are separated by 10.5 and $6.7 \mathrm{kpc}$ for 'a'- 'b' and 'b'-'c', respectively.

Except for the center of ' $b$ ' which shows absorption lines, all other objects show strong emission lines. The metallicity is lower than expected for an advanced merger of this luminosity, ranging between $12+\log (\mathrm{O} / \mathrm{H})=8.5$ and 8.6.

\section{B.7 AM 0748-665}

(Fig. 42) With a systemic velocity of $23500 \pm 200$ this system is the second most distant in our sample. It extends over more than $120 \mathrm{kpc}$ and the nuclei of 'A' and ' $\mathrm{B}$ ' are separated by about $47 \mathrm{kpc}$. ' $\mathrm{B}$ ' clearly is a massive elliptical galaxy, while the type of 'A' is not entirely clear. It could have been a spiral galaxy whose disk was disrupted by the interaction with ' $\mathrm{B}$ ' and is forming the tidal features of the system: 
The bridge between the two nuclei and a tidal tail, which is visible eastwards and north of 'A' as a faint plume (see Fig. 12 of Paper I). 'A' and 'B' show absorption line spectra with red continuum typical for early type galaxies.

In Paper I we designated five knots ' $a$ ' to ' $\mathrm{e}$ ' and selected the outermost two ('d' and ' $\mathrm{e}$ ') and the condensation ' $\mathrm{a}$ ' in the middle of the bridge as TDG candidates. Due to their faint surface brightness none of the knots ' $b$ ' to ' $e$ ' was detected in our spectroscopy.

\section{B.8 AM 1054-325}

(Fig. 43) 'A' and 'B' are interacting with a velocity difference of $\sim 80 \mathrm{~km} \mathrm{~s}^{-1}$, while ' $C$ ' is a background object. Despite the red colors of the nucleus of ' $B$ ' we observe very strong emission lines in that region, an indication of strong star formation.

In Paper I we had selected the outmost knots ' $\mathrm{g}$ ' through ' $\mathrm{k}$ ' and ' $\mathrm{m}$ ' as good candidates for TDGs. All of them are clearly extended, and have luminosities from $M_{B}=-10.4$ up to $-14.4 \mathrm{mag}$. All these regions are sites of very active star formation, showing strong emission lines from which we derive oxygen abundances around 1/4 solar. Some of the knots seem to have a double structure, splitting e.g. ' $\mathrm{g}$ ' and ' $h$ ' into two smaller knots. While the components of ' $\mathrm{g}$ ' have similar velocities their metallicities show a significant difference of roughly 0.2 dex. The components of ' $h$ ', on the other hand, have very similar metallicities but the velocity of the northern component is $400 \mathrm{~km} \mathrm{~s}^{-1}$ higher. This probably means that both knots are not single entities, but instead projections of two components each.

Unfortunately we did not take a spectrum of the second brightest knot ' $\mathrm{e}$ ' in 'A'. As we see several projection effects and strong velocity gradients within 'A' one could speculate, that ' $A$ ' actually is a merger by itself, with the progenitors designated as ' $A_{\text {nucl }}$ and ' $\mathrm{e}$ ' in Paper I. If true, the strong interaction between them together with the influence of ' $B$ ' could be the cause of the shock ionization we see in the emission line ratios of ' $A_{\text {nucl }}$ ' and the southern part of ' $g$ ' (see Fig. 30), as well as of the strong starburst activity in 'A'.

\section{B.9 AM 1159-530}

(Fig. 44) The center of 'A' shows an absorption line spectrum. Our central velocity of $4550 \mathrm{~km} \mathrm{~s}^{-1}$ is roughly consistent with the $4850 \pm 100 \mathrm{~km} \mathrm{~s}^{-1}$ given by Fairall (1983).

The TDG 'a' shows an oxygen abundance of 8.40 for both emission line regions, which is similar to the measured value of 8.49 in knot ' $q$ ', which is closer to the nucleus of ' $A$ '. Therefore ' $a$ ' is unlikely to be a preexisting galaxy, but is very probably being formed from the material expelled by ' $\mathrm{A}$ '. ' $\mathrm{a}$ ' also hosts the largest velocity gradient we observed in the tidal tails, $\Delta V_{\max }=440 \mathrm{~km} \mathrm{~s}^{-1}$ over an extent of 
$1.5 \mathrm{kpc}$. If completely attributed to rotation one could derive a dynamical mass of $6.7 \cdot 10^{10} M_{\odot}$ for this object using the virial theorem. But given the knotty appearance it is very unlikely to be a relaxed system, so the mass very likely is strongly overestimated. There seem to be two components in the velocity distribution, one that could represent the streaming motion of the tail, with a roughly constant velocity near $0 \mathrm{~km} \mathrm{~s}^{-1}$ (from -3 ." 5 to $-2^{\prime \prime}$ and from +3 .' 5 to 4. .' 5 ), and one which is likely due to internal motion within 'a', which dominates between -2 " and +3 .' 5 .

\section{B.10 AM 1208-273}

(Fig. 45) As already speculated in Paper I, this system consists of two galaxies 'A' and ' $\mathrm{C}$ ' which are located at the same distance, and the unrelated peculiar emission line galaxy 'B'. Donzelli \& Pastoriza (1997) have mixed up the velocities of 'A' and ' $\mathrm{B}$ ' in their paper. The pair 'A'+' $\mathrm{C}$ ' is actually closer to us, $V_{\mathrm{sys}} \approx 12430 \mathrm{~km} \mathrm{~s}^{-1}$, and ' $\mathrm{B}$ ' is in the background, $V_{\mathrm{B}}=14536 \pm 6 \mathrm{~km} \mathrm{~s}^{-1}$ (roughly consistent with the $14778 \mathrm{~km} \mathrm{~s}^{-1}$ of Donzelli \& Pastoriza 1997).

The spiral galaxy ' $\mathrm{A}$ ' shows small distortions so that it seems very unlikely that parts of the spiral arms can leave the disk and become TDGs. Most probably the much less luminous dwarf spiral ' $\mathrm{C}$ ' is the interacting partner.

The oxygen abundance measured in the disk of ' $\mathrm{A}$ ' is in the range $8.3<12+$ $\log (\mathrm{O} / \mathrm{H})<8.5$. That means we again have already used the correct metallicity for the models in Paper I.

\section{B.11 AM 1237-364}

(Fig. 46) The galaxies ' $\mathrm{B}$ ' and ' $\mathrm{C}$ ' are companions of ' $\mathrm{A}$ ' as already estimated from their blue colors, with velocity differences and projected distances to 'A' of $450 \mathrm{~km} \mathrm{~s}^{-1} / 24 \mathrm{kpc}$ and $170 \mathrm{~km} \mathrm{~s}^{-1} / 40 \mathrm{kpc}$, respectively. Both show low oxygen abundances, $12+\log (\mathrm{O} / \mathrm{H})_{\mathrm{B}}=7.50 \pm 0.08$ and $12+\log (\mathrm{O} / \mathrm{H})_{\mathrm{C}}=7.90 \pm 0.10$. The observed knots ' $d$ ', ' $e$ ', and ' $\mathrm{g}$ ' are associated with 'A' as well. But while 'd' and 'e' show typical metallicities of outer spiral disks, 'g' has a lower metallicity of $12+\log (\mathrm{O} / \mathrm{H})=7.72 \pm 0.06$.

\section{B.12 AM 1324-431}

(Fig. 47) Both nuclei 'A' and 'B' show strong emission lines and have similar velocities $\left(\Delta V \approx 80 \mathrm{~km} \mathrm{~s}^{-1}\right)$, roughly consistent with the literature value of Fisher et al. (1995). Their projected separation is $24 \mathrm{kpc}$. While ' $A$ ' has near solar metallicity $12+\log (\mathrm{O} / \mathrm{H})=8.71 \pm 0.05$, ' $\mathrm{B}$ ' has lower abundance $12+\log (\mathrm{O} / \mathrm{H})$ $=8.33 \pm 0.05$, and the emission line region between both nuclei has an intermediate value $12+\log (\mathrm{O} / \mathrm{H})=8.52 \pm 0.06$. 
The abundance of the one detected knot ' $c$ ' is higher than expected, but could only be estimated with large errors: $12+\log (\mathrm{O} / \mathrm{H})=8.66 \pm 0.22$.

Both observed velocity gradients are probably due to streaming motions between and around the two nuclei, and have no relation to TDGs.

\section{B.13 AM 1325-292}

(Fig. 48) In Paper I, we measured colors for the two blue TDG candidates 'a' and ' $b$ ' in this spiral-elliptical interacting system which could only be fit with models of low metallicity, although this seemed very unlikely given the nature of the parent galaxies. The spectra of the knots are of very low $\mathrm{S} / \mathrm{N}$ and the only visible features are very weak $\mathrm{H} \alpha$ emission-lines. However, the blue colors of the knots remain a mystery, just as the need for low metallicity models.

\section{B.14 AM 1353-272}

This system has been re-observed with FORS2 on the VLT. The results from these observations are included in the sample analyzed in this paper but its detailed discussion is subject of a different paper (Weilbacher et al. 2002).

Note that in Table 3 of Paper I we confused the velocities of ' $A$ ' and ' $B$ ' while the correct values were listed in the text. With the supplementary data available now, we can determine new central velocities $V_{\mathrm{A}}=11935 \pm 30 \mathrm{~km} \mathrm{~s}^{-1}$ and $V_{\mathrm{B}}=$ $11790 \pm 50 \mathrm{~km} \mathrm{~s}^{-1}$.

\section{References}

Arp, H.C., Madore, B.F., 1987, A Catalogue of Southern Peculiar Galaxies and Associations, Cambridge University Press, Cambridge

Barnes, J.E., Hernquist, L., 1998, ApJ 495, 187

Bergwall, N.A.S., Ekman, A.B.G., Lauberts, A., et al., 1978, $A \& A S$ 33, 243

Bomans, D.J., Weis, K., Tüllmann, R., Dettmar, R.-J., 2001, ApSSS 277, 51

Cairós, L.M., Caon, N., Vílchez, J., González-Pérez, J., Muñoz-Tuñón, C., 2001, ApJS 136, 393

Corwin, H.G., de Vaucouleurs, A., de Vaucouleurs, G., 1985, Southern galaxy catalogue, University of Texas Monographs in Astronomy, Austin: University of Texas, 1985 
Paper II.

Donzelli, C.J., Pastoriza, M.G., 1997, ApJS 111, 181

Dopita, M.A., Kewley, L.J., Heisler, C.A., Sutherland, R.S., 2000, ApJ 542, 224

Duc, P.-A., Brinks, E., Springel, V., et al., 2000, AJ 120, 1238

Duc, P.-A., Brinks, E., Wink, J.E., Mirabel, I.F., 1997, A\&A 326, 537

Edmunds, M.G., Pagel, B.E.J., 1984, MNRAS 211, 507

Fairall, A.P., 1983, MNRAS 203, 47

Ferguson, A.M.N., Gallagher, J.S., Wyse, R.F.G., 1998, AJ 116, 673

Fisher, K.B., Huchra, J.P., Strauss, M.A., et al., 1995, ApJS 100, 69

Gallagher, J.S., Hunter, D.A., Bushouse, H., 1989, AJ 97, 700

Heckman, T.M., Armus, L., Miley, G.K., 1990, ApJS 74, 833

Hibbard, J.E., Guhathakurta, P., van Gorkom, J.H., Schweizer, F., 1994, AJ 107, 67

Hibbard, J.E., van der Hulst, J.M., Barnes, J.E., Rich, R.M., 2001, AJ 122, 2969

Hunsberger, S.D., Charlton, J.C., Zaritsky, D., 1996, ApJ 462, 50

Iglesias-Páramo, J., Vílchez, J.M., 2001, ApJ 550, 204

Jiménez-Vicente, J., Battaner, E., 2000, A\&A 358, 812

Kennicutt, R.C., Edgar, B.K., Hodge, P.W., 1989, ApJ 337, 761

Marlowe, A.T., Meurer, G.R., Heckman, T.M., Schommer, R., 1997, ApJS 112, 285

Martin, C.L., 1998, ApJ 506, 222

Mathewson, D.S., Ford, V.L., 1996, ApJ 107, 97

Mendes de Oliveira, C., Plana, H., Amram, P., Balkowski, C., Bolte, M., 2001, AJ 121, 2524

Mirabel, I.F., Dottori, H., Lutz, D., 1992, A\&A 256, L19

Mirabel, I.F., Lutz, D., Maza, J., 1991, A\&A 243, 367

Pettini, M., Shapley, A.E., Steidel, C.C., et al., 2001, ApJ 554, 981 
Pilyugin, L.S., 2000, A\&A 362, 325

Pilyugin, L.S., 2001, A\&A 369, 594

Pleuss, P.O., Heller, C.H., Fricke, K.J., 2000, A\&A 361, 913

Richer, M.G., McCall, M.L., 1995, ApJ 445, 642

Schlegel, D.J., Finkbeiner, D.P., Davis, M., 1998, ApJ 500, 525

Schweizer, F., 1978, in: Structure and Properties of Nearby Galaxies (eds. E.M. Berkhuisen, R. Wiebilinski), 279

Scoville, N.Z., Polletta, M., Ewald, S., et al., 2001, AJ 122, 3017

Shields, G.A., 1990, ARA\&A 28, 525

van Zee, L., Salzer, J.J., Haynes, M.P., O’Donoghue, A.A., Balonek, T.J., 1998, AJ 116, 2805

Weilbacher, P.M., Duc, P.-A., 2001, in: Dwarf Galaxies and their Environment (eds. K.S. de Boer, R.-J. Dettmar, U. Klein), 269-272

Weilbacher, P.M., Duc, P.-A., Fritze-von Alvensleben, U., Martin, P., Fricke, K.J., 2000, A\&A 358, 819

Weilbacher, P.M., Fritze-von Alvensleben, U., 2001, in: Dwarf Galaxies and their Environment (eds. K.S. de Boer, R.-J. Dettmar, U. Klein), 287-290

Weilbacher, P.M., Fritze-von Alvensleben, U., Duc, P.-A., 2001, in: Extragalactic Star Clusters (eds. E.K. Grebel, D. Geisler, D. Minniti), vol. 207 of ASP Conf. Ser., in press

Weilbacher, P.M., Fritze-von Alvensleben, U., Duc, P.-A., Fricke, K.J., 2002, ApJ 579, L79

West, R.M., Surdej, J., Schuster, H.-E., et al., 1981, A\&AS 46, 57

Whitford, A.E., 1958, AJ 63, 201

Whitmore, B.C., Zhang, Q., Leitherer, C., et al., 1999, AJ 118, 1551

Xu, C., Sulentic, J.W., Tuffs, R., 1999, ApJ 512, 178

Zaritsky, D., Kennicutt, Jr., R.C., Huchra, J.P., 1994, ApJ 420, 87 
Paper II. 


\section{Paper III}

\section{Burst Properties: Optical-NIR Photometry and Models}

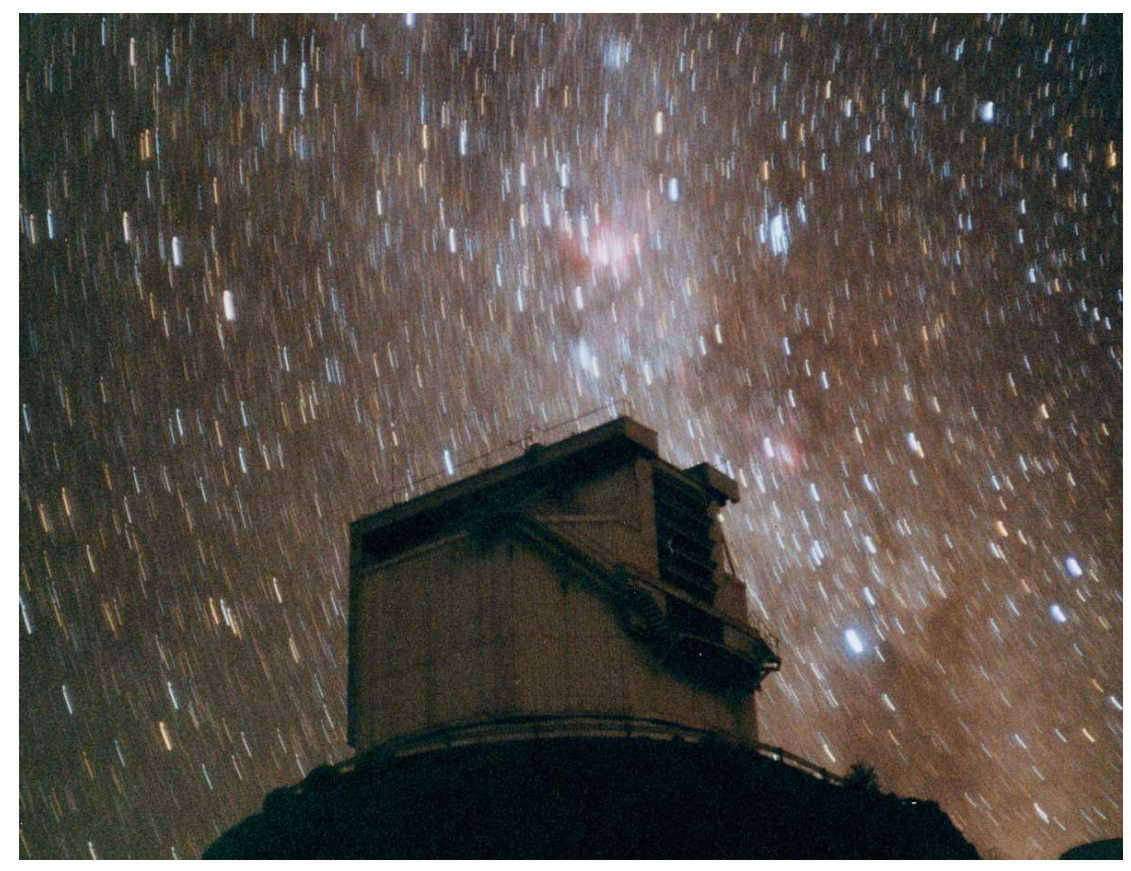



This paper is in preparation for

Astronomy \& Astrophysics.

\title{
Tidal Dwarf Candidates in a Sample of Interacting Galaxies
}

\section{Near-infrared photometry and evolutionary synthesis modeling *}

\author{
P. M. Weilbacher ${ }^{1}$, U. Fritze-v. Alvensleben ${ }^{1}$, P. Papaderos ${ }^{1}$, P.-A. Duc ${ }^{2}$ \\ ${ }^{1}$ Universitäts-Sternwarte, Geismarlandstr. 11, 37083 Göttingen, Germany, \\ \{weilbach, ufritze, papade\} @uni-sw.gwdg.de, \\ ${ }^{2}$ CNRS URA 2052 and CEA, DSM, DAPNIA, Service d'Astrophysique, Centre d'Etudes de Saclay, \\ 91191 Gif-sur-Yvette Cedex, France, paduc@cea.fr
}

\begin{abstract}
We present deep near-infrared (NIR) imaging of a sample of 14 interacting galaxies. In previous papers of this series we selected and analyzed a sample of 41 candidates for Tidal Dwarf Galaxies (TDGs), visible in these systems as star-forming knots positioned in the tidal features. To better constrain the properties of the stellar populations in these TDG candidates, we compute a grid of new spectrophotometric evolutionary synthesis models of a starburst on top of an underlying mixed-age population. The data presented here and in the previous papers allows to derive observed optical-NIR spectral energy distributions along with $\mathrm{H} \beta$ equivalent widths for many knots. From the comparison with the models we assess the properties of the TDG candidates. In particular, the mass ratio of the young stars to the old population inherited from the parent galaxy is crucial for better understanding the formation process of TDGs. For our sample this analysis suggests that more TDGs are built from stellar clumps than from giant gas clouds condensations. Additionally, we derive the current star formation rate and the stellar mass of these TDG candidates.

Key words: Galaxies: formation - Galaxies: interactions - Galaxies: dwarf Galaxies: photometry - Galaxies: evolution
\end{abstract}

*Based on observations collected at the European Southern Observatory, La Silla, Chile (ESO No 66.B-0055). 


\section{Introduction}

The formation of Tidal Dwarf Galaxies (TDGs) is a phenomenon which has been recognized to be important in many galaxy interactions. One of the key questions to be addressed in the context of TDG formation is, whether these massive condensations are born from instabilities in the gas phase of the tidal tails or if they start from stellar condensations. These scenarios have been put forward in dynamical simulations by Barnes \& Hernquist (1992) on the one hand and Elmegreen et al. (1993) on the other hand. Since then, several observational studies have established th presence of starburst activity in TDG candidates with different contributions by old and young stars. The most extreme cases have been observed in NGC 5291, where Duc \& Mirabel (1998) report the detection of TDGs made up of only young stars and gas, and Arp 245, where the TDG NGC $2992 \mathrm{~N}$ is believed to have only up to $2 \%$ of young stars built in the current star-formation episode (Duc et al. 2000).

In our previous papers (Weilbacher et al. 2000, 2002a, Papers I and II, respectively) we have compiled a sample of 14 interacting galaxies which contain numerous knots in their tidal features, some of which may be true TDGs. In Paper I we present optical photometry of the individual knots and - using evolutionary synthesis models identify promising TDG candidates against background galaxies. A first attempt to characterize the starburst properties of the knots from their optical colors alone was made using photometric evolutionary synthesis models. In Paper II, spectroscopy was presented and used to give additional information on the knots; the latter is necessary as input for the spectral evolutionary synthesis models, which was still missing in Paper I. This data allowed us to determine $\mathrm{H} \alpha$ fluxes and equivalent widths and to derive the metallicity of the knots. In particular, all TDG candidates identified from the comparison with the models for which a spectroscopic redshift could be measured, were confirmed to be physically associated with the interacting system.

In this paper, we present near-infrared (NIR) images and photometry of the systems in our sample. Regarding the comparison with the models it is important to derive the spectral energy distribution (SED) over a wider wavelength range, which better characterizes the stellar population. Inclusion of the NIR bands, in addition to the optical photometry, is of great importance in particular for constraints on the relative mass contribution of old stars inherited from the progenitor galaxy with respect to the young stars formed in the current star formation episode. This approach also allows to derive the current star formation rate with better precision than from the $\mathrm{H} \alpha$ luminosity alone (Weilbacher \& Fritze-von Alvensleben 2001). With all the photometric data available, we can now make a detailed comparison of the starburst models with the observed spectrophotometry collected here to determine the evolutionary status of the TDG candidates. 
Table 20: Observing summary

\begin{tabular}{ccc}
\hline Object & Filter & $\begin{array}{c}\text { Exptime } \\
\text { on target }\end{array}$ \\
\hline AM 0529-565 & $H$ & $30 \mathrm{~min}$ \\
AM 0537-292 & $H$ & $30 \mathrm{~min}$ \\
AM 0547-244 & $H / K s$ & $50 / 50 \mathrm{~min}$ \\
AM 0547-474 & $H$ & $25 \mathrm{~min}$ \\
AM 0607-444 & $H$ & $30 \mathrm{~min}$ \\
AM 0748-665 & $H$ & $40 \mathrm{~min}$ \\
AM 1054-325 & $H$ & $30 \mathrm{~min}$ \\
AM 1159-530 & $J / H / K s$ & $30 / 40 \mathrm{~min}$ \\
AM 1208-273 & $H$ & $30 \mathrm{~min}$ \\
AM 1237-364 & $H$ & $65 \mathrm{~min}$ \\
AM 1324-431 & $H$ & $30 \mathrm{~min}$ \\
AM 1325-292 & $H$ & $15 \mathrm{~min}$ \\
AM 1353-272 & $J / H / K s$ & $40 / 54 / 43 \mathrm{~min}$ \\
\hline
\end{tabular}

After describing our data acquisition and analysis methods for the NIR data in Sect. 2 and the specific features of our spectrophotometric models in Sect. 3, we describe the procedure to compare the observations with the models in Sect. 4. In Sect. 5 we present and discuss the results for our sample of TDG candidates. A short summary of the results is given in Sect. 6.

\section{Observations and data analysis}

We obtained NIR images of the sample of 14 interacting galaxies presented in $\mathrm{Pa}$ per II. All objects were taken from the Catalog of Southern Peculiar Galaxies (Arp $\&$ Madore 1987). The NIR observations were carried out with the SOFI instrument at the 3.58m ESO NTT at La Silla during 2 nights in February 2001 (see Table 20). SOFI has a $1 \mathrm{k} \times 1 \mathrm{k}$ Hawaii array with a pixel size of $0 . \mathrm{p}^{\prime \prime} 292 \mathrm{px}^{-1}$ on the sky, yielding a usable field of view of $\sim 4.5 \times 4.5$. Due to time constraints most objects were observed in one filter only; we preferred to use $H$-band data, because of the higher $\mathrm{S} / \mathrm{N}$ as compared with $K s$. Most objects have sizes up to $2^{\prime} \times 2^{\prime}$ and the imaging was done efficiently with on-chip jittering. In the cases of the three larger object pairs AM 0547-244, AM 1054-325, and AM 1325-292 we used the jitter method with offset sky fields between each on target integration. The weather was clear in the first night and photometric in the second night. In both nights several photometric standard stars from the catalog of Persson et al. (1998) were observed; the zero- 
point variations were $<0.02 \mathrm{mag}$, so the data from both nights was combined for calibration.

\subsection{Data reduction}

Data reduction was performed with the ESO eclipse package. First, we removed effects created by the detector and read-out: the odd-even column effect that was visible in two quadrants and the electrical "ghost". Afterwards the standard reduction steps were performed: all object- and sky-frames were flat-fielded. A sky-frame was then built from up to 15 frames (only sky frames for jitter+offset) nearest in time and then subtracted from each object frame. All object frames were then registered and averaged, rejecting the highest and lowest pixels during combination. The parameters for these steps were tuned to each observed field and then performed automatically by the jitter command of the eclipse package.

We also used a NIR reduction package for the MIDAS environment developed in Göttingen by K. Noeske (see e.g. Noeske et al. 2002). It allows to visually check each reduction step and to mask out bright stars before sky-combination. This procedure gave better results for problematic fields and was used in the cases of AM 0547-474 and AM 1237-364.

\subsection{Photometry}

Further analysis was done in the IRAF environment ${ }^{1}$. As we are interested in the optical-NIR colors of the regions already measured on the optical images, we registered and PSF-matched the NIR images using as reference the respective $V$-band image of the same field on which the polygonal apertures were defined (Paper I). Before performing the photometry, we masked out foreground stars using IRAF's imedit task. For the photometry, the polyphot task was used with the same integration aperture as the corresponding optical image. For objects far away from the tidal features, the magnitudes within circular apertures were measured with phot. We corrected the magnitudes in all filters using the Galactic extinction from Schlegel et al. (1998) as listed in the NED ${ }^{2}$.

Several knots in the tidal tails are only detected at a low S/N level in the NIR. In those cases our data yields an upper limit on the true magnitude, and reveals several knots where no NIR counterpart is visible. As an example, the field of AM 0527292 is shown in Fig. 49. For these cases we simulated several galaxy images with different sizes, scale lengths, and magnitudes on two $H$ band frames (with total

\footnotetext{
${ }^{1}$ IRAF is written and supported by the IRAF programming group of the National Optical Astronomy Observatories (NOAO).

${ }^{2}$ The NASA/IPAC Extragalactic Database (NED) is operated by the Jet Propulsion Laboratory, California Institute of Technology.
} 


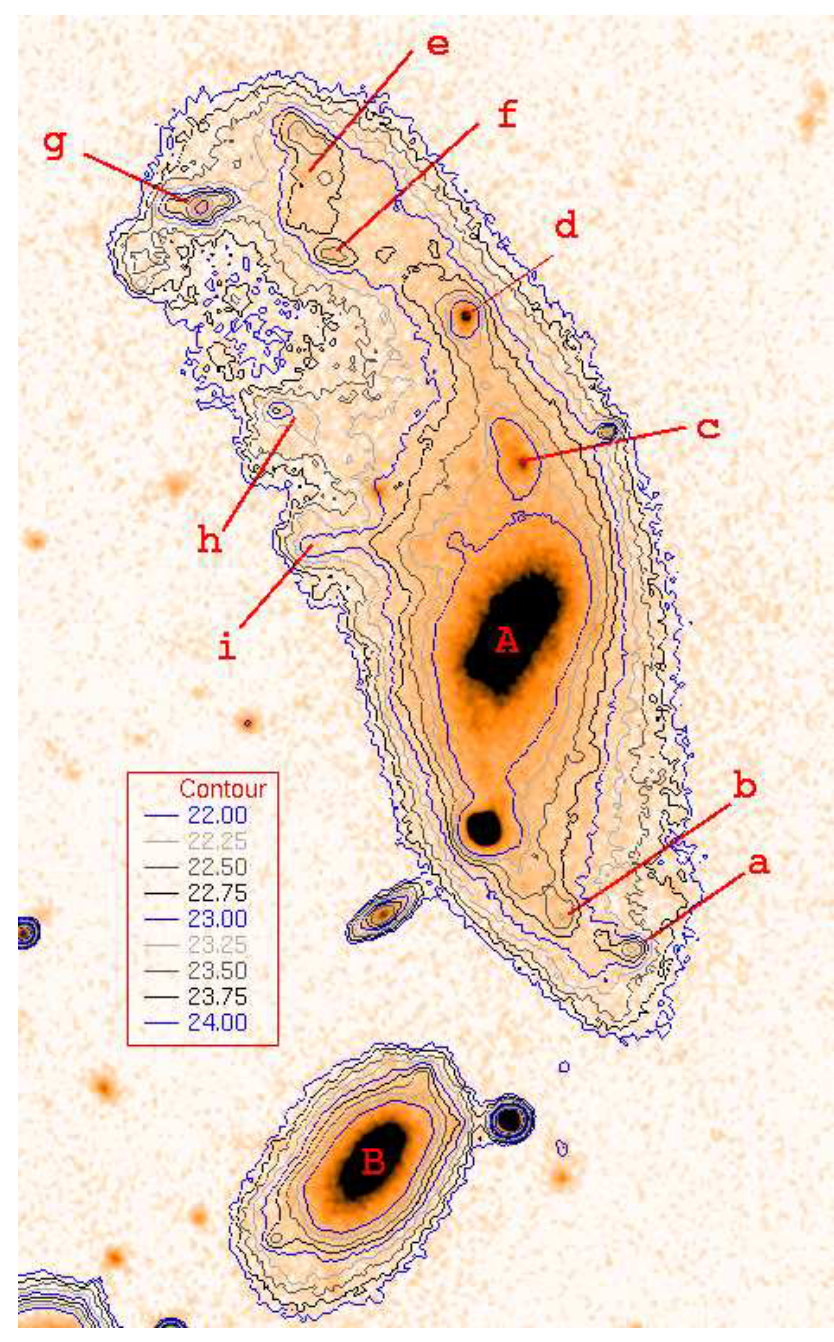

Figure 49: Example on the detectability of TDG candidates on NIR images ( $H$ band of AM 0537-292, contours are from the $B$-band image). While the knots ' $\mathrm{c}$ ' and 'd' are visible, knots ' $h$ ' and 'i' are only marginally detected.

exposure times of 54 and $30 \mathrm{~min}$ ) using the IRAF package artdata. Due to the wide range of observed morphologies for our knots, we found a range in limiting magnitude of $H=20.8 \ldots 21.3$ mag when measuring the magnitudes of galaxies simulated with different parameters. Note, that this is not directly comparable with a completeness limit used in e.g. surveys and globular cluster studies, as the knots are not searched for by an automatic procedure. 
Table 21: Parameters of the model grid.

\begin{tabular}{crcc}
$\begin{array}{c}\tau_{\mathrm{B}} \\
{\left[10^{6} \mathrm{yr}\right]}\end{array}$ & $\begin{array}{c}\psi_{0} \\
{\left[M_{\odot} \mathrm{yr}^{-1}\right]}\end{array}$ & $B_{64} / b_{64}$ & $B_{35} / b_{35}$ \\
\hline 5.0 & 5 & $0.08 / 8 \%$ & $0.15 / 13 \%$ \\
5.0 & 20 & $0.33 / 25 \%$ & $0.61 / 38 \%$ \\
5.0 & 100 & $1.63 / 62 \%$ & $3.03 / 75 \%$ \\
5.0 & 500 & $8.14 / 89 \%$ & $15.2 / 94 \%$ \\
5.0 & 1000 & $16.3 / 94 \%$ & $30.3 / 97 \%$ \\
\hline
\end{tabular}

\section{Model description}

To interpret the photometric and spectroscopic results, we basically employ the same code for our evolutionary synthesis models as presented in Paper I - based on the current Geneva stellar tracks. We therefore restrict ourselves to the description of the differences of the current model implementation to the previous ones.

The initial mass function (IMF) from Salpeter (1955) is used in the mass range 0.15 to $120 \mathrm{M}_{\odot}$. As shown elsewhere (Krüger et al. 1995, Weilbacher \& Fritze-von Alvensleben 2001), gaseous emission is of great importance for the evolution of the optical and NIR broad-band colors during starbursts. Besides the gaseous continuum emission, we therefore now include new emission line ratios for low metallicity extragalactic environments and blue compact dwarf (BCD) galaxies from Izotov et al. $(1994,1997)$ and Izotov \& Thuan (1998). The emission line ratios in this sample seem to be representative for the dwarf galaxy population (Stasińska et al. 2001); the large number of objects observed in different metallicity ranges allows us to define new empirical emission line ratios for the two low metallicities, $Z_{1}=0.001$, $Z_{2}=0.004$. For the higher metallicities, $Z_{3}=0.008$ and $Z_{4}=0.020 \approx Z_{\odot}$, no large, consistent sample with a sufficient number of observed lines seems to be available, we therefore continue to use the ratios of Stasińska (1984) which are in good agreement with galactic $\mathrm{H}$ II region observations. To be able to compute synthetic spectra, we have included the spectral library of Lejeune et al. $(1997,1998)$. The spectral synthesis determines the stellar continuum. To this, the gaseous continuum is added. The $\mathrm{H} \beta$ luminosity is derived from the Lyman continuum production rate (see Paper I), and together with the continuum this yields the $\mathrm{H} \beta$ equivalent width (EW).

Aside from the metallicity and the IMF, the only input parameter for the models is the star formation (SF) history. To describe possible SF histories of a TDG, we have created a grid of models using starbursts of different strengths on top of an underlying "old" stellar population (see Tab. 21). To give a more realistic starburst model, we now use a bell-shaped SF history with a SF rate rising and falling on a 
characteristic timescale $\tau_{\mathrm{B}}=5 \cdot 10^{6} \mathrm{yr}$ during the burst. This allows us to also find those TDGs where the SF is still increasing, i.e. those currently experiencing the onset of the burst.

To model the mixed-age stars in a TDG (often simply called "old" in contrast to the young population formed in the current starburst), inherited from the spiral disk, we use a SF rate proportional to the gas mass, for which - after evolution over a Hubble time - models reach colors and gas masses of an average spiral galaxy of type Sc. To easily get a wide range of burst strengths, we vary the total mass of the underlying old population. We create models where the starburst occurs when $35 \%$, and $64 \%$ of the total gas reservoir was already turned into stars. As some TDGs could also be built from pure gas, we include models creating only a starburst population, i.e. models without old component.

We use the same definition for the burst strength $b$ as in Paper I, where $b$ is the ratio of the mass of stars created in the starburst vs. the mass of stars ever formed:

$$
b=\frac{M(\text { young })}{M(\text { old }+ \text { young })}
$$

With this definition, an entirely young starburst population is described by a burst strength of $b=1.0$ or $100 \%$. The burst strength for the models with an old population, after the end of the burst, i.e. at the time $t=t_{\max }+2 \cdot \tau_{\mathrm{B}}$ is given in Tab. 21; the index in the table denotes the percentage of gas in the model at the onset of the burst. Another alternative definition for the burst strength frequently used is

$$
B=\frac{M(\text { young })}{M(\text { old })}
$$

which we also list in Tab. 21 at the same time after the burst maximum. With this definition the models without old component ( $b=1.0$ in Eq. 1 ) have $B=\infty$.

\section{Comparison observations-models}

With the available observations we can now use the metallicity, the $\mathrm{H} \beta$ equivalent width $\operatorname{EW}(\mathrm{H} \beta)$, and up to 6 optical and NIR magnitudes to find the best fitting model for each observed knot. The simple method used in Paper I - visual selection of the best model from the position in a two-color diagram - cannot be used here. Instead, we select the best model numerically, comparing the observed spectral energy distribution and $\mathrm{EW}(\mathrm{H} \beta)$ to all timesteps - beginning with the start of the burst. This is repeated for all models of the grid.

If the $\mathrm{EW}(\mathrm{H} \beta)$ of the knot is known, the first step is to select only those timesteps within a model, where the EW is close to the observed value, i.e. within a $10 \%$ estimated model uncertainty from the observed values including their $1 \sigma$ errors. This 


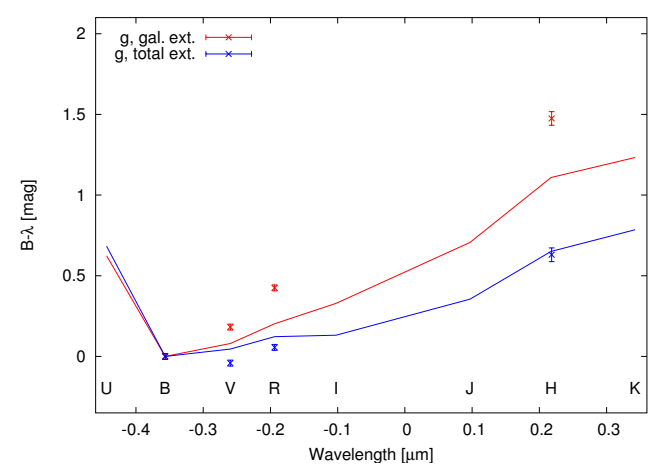

Figure 50: Normalized SEDs and fits of the TDG candidate ' $\mathrm{g}$ ' in the system AM 0537-292.

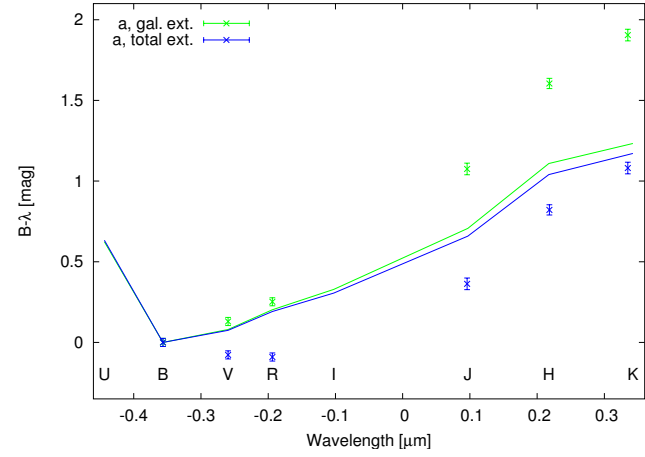

Figure 51: Normalized SEDs and fits of the TDG candidate ' $a$ ' in the system AM 1159-530. Here, the model grid does not provide a good reproduction of the SED within the ranges of matching $\mathrm{EW}(\mathrm{H} \beta)$.

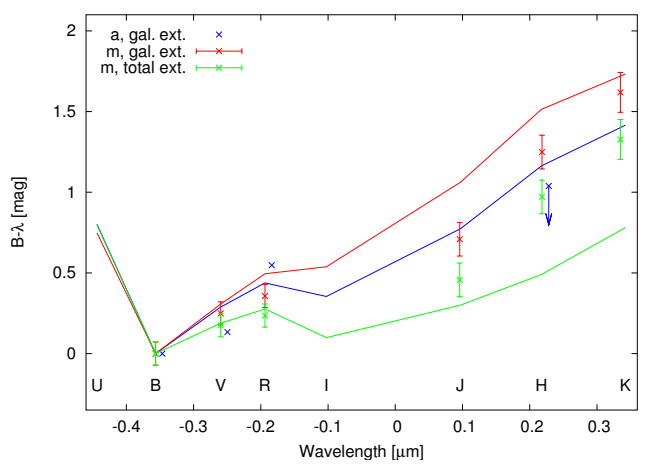

Figure 52: Normalized SEDs and fits of TDG candidates ' $a$ ' and ' $m$ ' in the system AM 1353-272. The wavelengths for knot 'a' have been slightly shifted to make the datapoints visible.

typically defines two windows within a single model, where the $\mathrm{EW}(\mathrm{H} \beta)$ "intersects" the observed value (see Weilbacher \& Fritze-von Alvensleben 2001). For all timesteps within such a range we compute a least squares based score for all available broad band filters and for the $\mathrm{EW}(\mathrm{H} \beta)$, assigning to the latter a higher weight than to the broadband measurements. If no Balmer line EW has been measured, the score is only computed from the available filters. This is repeated for all models with metallicities matching the observed oxygen abundance. If no metal abundance has been observed, we compute the score of all models. Finally, the model with the best score is selected as the best fit model. 
For the objects with only a marginal or no detection in the NIR, we used the upper limit determined in Sect. 2.2 with an estimated error for the $H$-band to compare to the models. In these cases, the objects could be bluer that the upper limit determined for the colors, which means that they could be younger or experience a stronger burst that indicated by our fit.

Whenever an extinction larger than the galactic value has been spectroscopically determined for a knot, we also derive a model fit for the dereddened magnitudes. The observed total extinction is very likely an upper limit with regard to the colors of a whole knot which in general is larger than the spectroscopic slit; hence, the true best fit will likely be between the values derived from both fits. As the age within each model is chiefly determined by comparison with the observed $E W(H \beta)$, which does not depend on the assumed extinction, we derive similar values for the burst ages between the two model fits with different extinction values. Only the result from the better fit is included in Tab. 23.

The best fit gives a reasonably good representation of the true SED of the respective object within our model grid. A check showed that the next best model fit either has similar properties or gives a much worse fit. This means that the age we derive should be uncertain by only about $10 \%$ and the burst strength $b$ can be inferred with an accuracy of $5 \%$. The derived SFR is strongly coupled with the age determination and has a comparable relative error. The error for the derived stellar mass is estimated to be around $15 \%$. One also has to be aware of the discreteness of the models grid, e.g. that there is a gap between burst strengths of $60 \%$ and $40 \%$ (see e.g. Fig. 51).

\section{Results and discussion}

In Papers I and II we established a sample of 44 TDG candidates from optical imaging. Three of those were shown to have metallicities 0.6 dex lower than the mean value of $12+\log (\mathrm{O} / \mathrm{H})=8.34$ derived for our sample. While some of the objects still lack spectroscopic confirmation of their physical association with the interacting system, we start here from a sample of 41 TDG candidates. We will discuss if the 13 objects for which the spectra showed internal kinematics (Paper II) show any specific properties different from those of the rest of the sample.

\subsection{Photometric properties}

Table 22 lists the properties relevant for the comparison with the models: the equivalent width of the $\mathrm{H} \beta$ emission and the magnitudes with photometric errors in $B$, $J, H$, and $K s$ measured from the NTT observations, as well as the distance moduli 
Table 22: NIR photometric magnitudes of the 41 TDG candidates.

\begin{tabular}{|c|c|c|c|c|c|c|}
\hline Objectname & $\mathrm{EW}(\mathrm{H} \beta)^{1}$ & $\begin{array}{l}\mathrm{m}-\mathrm{M}^{2} \\
{[\mathrm{mag}]}\end{array}$ & $\begin{array}{c}B^{3} \\
{[\mathrm{mag}]}\end{array}$ & $\begin{array}{c}J \\
{[\mathrm{mag}]}\end{array}$ & $\begin{array}{l}H \\
{[\mathrm{mag}]}\end{array}$ & $\begin{array}{c}K s \\
{[\mathrm{mag}]}\end{array}$ \\
\hline$\overline{\mathrm{AM} 0529-565 a}$ & - & 33.88 & $22.36 \pm 0.04$ & - & $19.26 \pm 0.06$ & - \\
\hline AM $0529-565 b$ & - & 33.88 & $23.19 \pm 0.03$ & - & $19.91 \pm 0.07$ & - \\
\hline AM $0529-565 \mathrm{~g}$ & - & 33.88 & $22.20 \pm 0.02$ & - & $\sim 20.8$ & - \\
\hline AM $0529-565 i$ & - & 33.88 & $21.17 \pm 0.01$ & - & $19.59 \pm 0.06$ & - \\
\hline AM 0537-292a & $-159.7 \pm 5.8$ & 33.58 & $20.83 \pm 0.01$ & - & $\sim 20.8$ & - \\
\hline AM 0537-292d & $-12.2 \pm 1.2$ & 33.58 & $19.59 \pm 0.00$ & - & $17.70 \pm 0.03$ & - \\
\hline AM 0537-292e & - & 33.58 & $21.01 \pm 0.01$ & - & $19.45 \pm 0.06$ & - \\
\hline AM 0537-292f & - & 33.58 & $21.06 \pm 0.01$ & - & $19.19 \pm 0.05$ & - \\
\hline AM $0537-292 \mathrm{~g}$ & $-34.3 \pm 2.6$ & 33.58 & $19.70 \pm 0.00$ & - & $18.23 \pm 0.03$ & - \\
\hline AM 0547-244a & $-24.8 \pm 3.7$ & 36.23 & $19.15 \pm 0.01$ & - & $\sim 20.8$ & - \\
\hline AM $0547-244 b$ & $-24.5 \pm 2.2$ & 36.23 & $19.05 \pm 0.01$ & - & $17.74 \pm 0.03$ & $17.24 \pm 0.03$ \\
\hline AM 0547-244d & - & 36.23 & $21.63 \pm 0.06$ & - & $\sim 20.8$ & - \\
\hline AM 0607-444a & 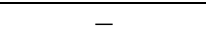 & 36.16 & $19.70 \pm 0.00$ & 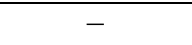 & $16.24 \pm 0.01$ & $16.66 \pm 0.46$ \\
\hline AM 0748-665a & - & 37.48 & $19.75 \pm 0.01$ & - & $17.63 \pm 0.03$ & - \\
\hline AM $0748-665 d^{4}$ & - & 37.48 & $18.22 \pm 0.00$ & - & $(15.57 \pm 0.01)$ & - \\
\hline АМ $0748-665$ e & - & 37.48 & $21.38 \pm 0.02$ & - & $\sim 20.8$ & - \\
\hline AM 1054-325g & $-79.0 \pm 3.7$ & 33.62 & $19.13 \pm 0.00$ & - & $17.57 \pm 0.03$ & - \\
\hline AM 1054-325h & $-61.3 \pm 10.1$ & 33.62 & $19.68 \pm 0.00$ & - & $19.43 \pm 0.06$ & - \\
\hline AM 1054-325j & $-116.1 \pm 1.9$ & 33.62 & $19.05 \pm 0.00$ & - & $15.29 \pm 0.01$ & - \\
\hline AM $1054-325 \mathrm{k}$ & - & 33.62 & $23.01 \pm 0.02$ & - & $>20.8$ & - \\
\hline AM $1054-325 \mathrm{~m}$ & - & 33.62 & $22.01 \pm 0.01$ & - & $\sim 20.8$ & - \\
\hline AM 1159-530a & $-33.9 \pm 3.0$ & 33.92 & $17.66 \pm 0.00$ & $16.58 \pm 0.02$ & $16.05 \pm 0.01$ & $15.75 \pm 0.02$ \\
\hline AM $1159-530 h$ & - & 33.92 & $20.35 \pm 0.01$ & $18.97 \pm 0.05$ & $18.44 \pm 0.04$ & $18.26 \pm 0.06$ \\
\hline AM 1324-431c & $-13.7 \pm 7.0$ & 35.77 & $20.75 \pm 0.01$ & - & $19.06 \pm 0.05$ & - \\
\hline AM 1324-431d & - & 35.77 & $21.46 \pm 0.01$ & - & $>20.8$ & - \\
\hline AM $1324-431$ e & - & 35.77 & $21.99 \pm 0.01$ & - & $>20.8$ & - \\
\hline AM 1324-431h & - & 35.77 & $21.67 \pm 0.01$ & - & $\sim 20.8$ & - \\
\hline AM 1325-292a & $-3.4 \pm 2.9$ & 33.89 & $18.50 \pm 0.00$ & - & $15.70 \pm 0.01$ & - \\
\hline AM $1325-292 b$ & - & 33.89 & $18.34 \pm 0.00$ & - & $15.42 \pm 0.01$ & - \\
\hline AM 1353-272a & $-321.7 \pm 18.1$ & 36.01 & $22.34 \pm 0.03$ & - & $>20.8$ & - \\
\hline AM $1353-272 b$ & $-98.3 \pm 4.0$ & 36.01 & $22.92 \pm 0.03$ & - & $\sim 20.8$ & - \\
\hline AM $1353-272 c$ & $-121.9 \pm 3.8$ & 36.01 & $22.44 \pm 0.02$ & - & $\sim 20.8$ & - \\
\hline AM $1353-272 d$ & $-150.0 \pm 4.2$ & 36.01 & $22.04 \pm 0.02$ & - & $\sim 20.8$ & - \\
\hline AM $1353-272 \mathrm{e}$ & $-153.9 \pm 22.2$ & 36.01 & $21.99 \pm 0.02$ & - & $>20.8$ & - \\
\hline AM 1353-272f & - & 36.01 & $23.18 \pm 0.03$ & - & $\sim 20.8$ & - \\
\hline AM 1353-272h & - & 36.01 & $23.50 \pm 0.03$ & - & $>20.8$ & - \\
\hline AM $1353-272 i$ & - & 36.01 & $23.89 \pm 0.04$ & $21.54 \pm 0.14$ & $21.13 \pm 0.15$ & $20.74 \pm 0.18$ \\
\hline AM $1353-272 k$ & $-27.4 \pm 5.4$ & 36.01 & $22.03 \pm 0.01$ & $20.86 \pm 0.10$ & $20.23 \pm 0.10$ & $19.97 \pm 0.12$ \\
\hline AM 1353-2721 & $-48.0 \pm 1.8$ & 36.01 & $20.77 \pm 0.01$ & $19.61 \pm 0.06$ & $19.07 \pm 0.06$ & $18.80 \pm 0.07$ \\
\hline AM $1353-272 m$ & $-225.9 \pm 4.8$ & 36.01 & $21.56 \pm 0.01$ & $20.85 \pm 0.10$ & $20.31 \pm 0.10$ & $19.94 \pm 0.12$ \\
\hline AM $1353-272 n$ & - & 36.01 & $21.71 \pm 0.03$ & - & $>20.8$ & - \\
\hline
\end{tabular}

${ }^{1}$ From the spectroscopic data of Paper II

${ }^{2}$ Using the distance given in Paper II

${ }^{3}$ From the optical photometry (Paper I)

${ }^{4}$ This object had to be deblended from the light of a nearby bright star; the derived magnitude is highly uncertain 
$m-M$. The $V$ and $R$ magnitudes are not repeated here, they can be looked up in Paper I.

From the 41 knots, 23 have been detected in the NIR with good S/N ratios. 12 further objects are detected on the NIR frames with S/N lower than 2, so that the photometry does not give reliable values. Their magnitudes are listed as approximately the detection limit $H \sim 20.8$ mag. The remaining 6 TDG candidates are not visible on any NIR image, their magnitude being fainter than the detection limit ( $H<20.8 \mathrm{mag}$ ). Knot AM 1353-272 $\mathrm{i}$ is an exception; it is sharply peaked, and can be reliably measured on the NIR frames despite its faint magnitude.

While at first glance the non-detection in the deep NIR images points to a very young stellar population without a large contribution by old stars, this has to be confirmed using fits to the SEDs of the objects. Table 23 summarizes the results of the comparison of the observed $\mathrm{H} \beta$ equivalent widths and the SEDs with the values from our model grid. There, we list the metallicity of the best fit model (using the notation from Sect. 3), the age and burst strength $b$ at the timestep of the best fit as well as the burst strength the modeled burst reaches near the end of the burst. We also derive the star formation rate and the stellar mass from the comparison with the best fit model.

\subsection{Properties derived from the models}

The models allow to draw several conclusions. First of all, we find matching models for all TDG candidates in our sample. If we define as good a fit that reproduces the observations to better than $0.25 \mathrm{mag}$, which - given the rough grid and the uncertainties of the models - is an attainable goal, we find only 7 knots (listed in the lower part of Tab. 23) for which no good fit can be found. Of these, 4 are detected in four filters $(B, V, R$, and $H)$ and the $\mathrm{EW}(\mathrm{H} \beta)$ is known. Because we force the fit procedure to ignore those points in the models where the EW is not within $10 \%$ of the observed values including error to derive a good age estimate, the best fit may not be the best fit to the observed colors. This could be problematic in particular for the knots in the system AM 1054-325, for which shocks contribute to the observed line emission (Paper II). As the colors are also strongly affected by the gaseous emission, it makes very little sense to recompute the fit without the restriction by the $\mathrm{EW}(\mathrm{H} \beta)$. Another restriction may be the observed oxygen abundance, which we use to preselect the model. If the metal abundance of an object lies near the limits of the respective metallicity range of the stellar tracks, the fit may be assigned a worse score than the model of the next lower or higher metallicity. While this will mostly affect the numerical score of the fit, the best fit with a different metallicity model could also change the results. However, no grid of stellar tracks with finer steps in metallicity is currently available avoid this problem. 
Table 23: Comparison of parameters of the model grid. The upper part lists reliable fits.

\begin{tabular}{|c|c|c|c|c|c|c|c|}
\hline Objectname $^{1}$ & $\mathrm{Z}$ & $\begin{array}{r}\text { age }^{2} \\
{[\mathrm{Myr}]}\end{array}$ & $\begin{array}{r}b[\%] \\
\text { current2 }\end{array}$ & $\times \tau_{\mathrm{B}}[M$ & $\begin{array}{c}\text { SFR } \\
{\left[\odot \mathrm{yr}^{-1}\right]}\end{array}$ & $\begin{array}{c}M_{\text {stellar }} \\
{\left[M_{\odot}\right]}\end{array}$ & $\mathrm{S} / \mathrm{N}^{3}$ \\
\hline AM 0529-565a & $\mathrm{Z4}$ & -16 & 0 & 25 & 0.00 & $2.4 \cdot 10^{7}$ & + \\
\hline AM 0529-565b & $\mathrm{Z4}$ & -19 & 0 & 25 & 0.00 & $1.3 \cdot 10^{7}$ & + \\
\hline AM 0529-565g & $\mathrm{Z} 1$ & -14 & 1 & 62 & 0.03 & $1.0 \cdot 10^{7}$ & o \\
\hline AM 0529-565i & $\mathrm{Z1}$ & 374 & 76 & 75 & 0.00 & $4.8 \cdot 10^{7}$ & + \\
\hline AM $0537-292 d^{4}$ & $\mathrm{Z} 2$ & 17 & 41 & 38 & 0.00 & $1.5 \cdot 10^{7}$ & + \\
\hline AM 0537-292e & $\mathrm{Z} 1$ & 370 & 64 & 62 & 0.00 & $6.0 \cdot 10^{7}$ & + \\
\hline AM 0537-292f & $\mathrm{Z} 1$ & 565 & 63 & 62 & 0.00 & $6.5 \cdot 10^{7}$ & + \\
\hline AM 0537-292g & $\mathrm{Z} 2$ & 21 & 100 & 100 & 0.01 & $4.7 \cdot 10^{6}$ & + \\
\hline AM 0547-244b & $\mathrm{Z2}$ & 14 & 15 & 13 & 0.06 & $2.5 \cdot 10^{8}$ & + \\
\hline AM 0547-244d & $\mathrm{Z3}$ & -15 & 3 & 94 & 3.82 & $2.2 \cdot 10^{8}$ & o \\
\hline AM 0607-444a & $\mathrm{Z4}$ & 2495 & 100 & 100 & 0.00 & $7.1 \cdot 10^{7}$ & + \\
\hline AM 0748-665a & $\mathrm{Z} 2$ & 74 & 40 & 38 & 0.00 & $2.7 \cdot 10^{9}$ & + \\
\hline (AM 0748-665d) & (Z1) & (284) & (8) & (8) & $(0.00)$ & $\left(1.7 \cdot 10^{10}\right)$ & $(+)$ \\
\hline AM 0748-665e & $\mathrm{Z} 3$ & -15 & 3 & 94 & 12.29 & $7.2 \cdot 10^{8}$ & o \\
\hline AM $1054-325 k$ & $\mathrm{Z} 1$ & -18 & 0 & 94 & 0.01 & $4.2 \cdot 10^{6}$ & - \\
\hline AM 1054-325m & $\mathrm{Z3}$ & -16 & 1 & 94 & 0.14 & $1.5 \cdot 10^{7}$ & o \\
\hline AM 1159-530a & $\mathrm{Z} 2$ & 13 & 9 & 8 & 0.07 & $2.8 \cdot 10^{8}$ & + \\
\hline AM 1159-530h & $\mathrm{Z} 2$ & -15 & 0 & 75 & 0.10 & $3.1 \cdot 10^{7}$ & + \\
\hline AM $1324-431 \mathrm{c}$ & $\mathrm{Z3}$ & 14 & 15 & 13 & 0.01 & $4.0 \cdot 10^{7}$ & + \\
\hline AM 1324-431e & $\mathrm{Z3}$ & -15 & 5 & 97 & 1.23 & $4.0 \cdot 10^{7}$ & - \\
\hline AM 1324-431h & $\mathrm{Z} 2$ & -5 & 2 & 8 & 0.48 & $1.0 \cdot 10^{8}$ & o \\
\hline AM $1325-292 b$ & $\mathrm{Z} 4$ & 415 & 13 & 13 & 0.00 & $5.4 \cdot 10^{8}$ & + \\
\hline AM 1353-272a & $\mathrm{Z} 2$ & -13 & 2 & 75 & 0.09 & $9.4 \cdot 10^{6}$ & - \\
\hline AM 1353-272b & $\mathrm{Z} 2$ & -14 & 0 & 25 & 0.03 & $4.1 \cdot 10^{7}$ & o \\
\hline AM 1353-272 $c^{4}$ & $\mathrm{Z} 2$ & 7 & 8 & 8 & 0.04 & $1.5 \cdot 10^{7}$ & o \\
\hline AM 1353-272d & $\mathrm{Z} 2$ & 5 & 13 & 13 & 0.04 & $5.3 \cdot 10^{6}$ & o \\
\hline AM $1353-272 e$ & $\mathrm{Z3}$ & 1 & 5 & 8 & 0.09 & $1.2 \cdot 10^{7}$ & - \\
\hline AM 1353-272f & $\mathrm{Z3}$ & -13 & 0 & 25 & 0.06 & $5.9 \cdot 10^{7}$ & o \\
\hline AM 1353-272h & $\mathrm{Z4}$ & 107 & 8 & 8 & 0.00 & $5.6 \cdot 10^{7}$ & o \\
\hline AM 1353-272i & $\mathrm{Z4}$ & -14 & 0 & 25 & 0.03 & $4.0 \cdot 10^{7}$ & + \\
\hline AM 1353-272k & $\mathrm{Z2}$ & 14 & 15 & 13 & 0.00 & $1.6 \cdot 10^{7}$ & + \\
\hline AM 1353-272l ${ }^{4}$ & $\mathrm{Z} 2$ & 12 & 9 & 8 & 0.05 & $1.1 \cdot 10^{8}$ & + \\
\hline AM 1353-272m & $\mathrm{Z} 2$ & -8 & 1 & 8 & 0.12 & $5.7 \cdot 10^{7}$ & + \\
\hline AM 1353-272n & $\mathrm{Z3}$ & -13 & 4 & 89 & 3.52 & $1.4 \cdot 10^{8}$ & - \\
\hline$\overline{\mathrm{AM} \mathrm{0537-292a}}$ & $\mathrm{Z2}$ & 5 & 97 & 97 & 0.01 & $2.1 \cdot 10^{5}$ & $\mathrm{O}$ \\
\hline AM 0547-244a & $\mathrm{Z2}$ & 15 & 95 & 94 & 0.02 & $2.7 \cdot 10^{7}$ & o \\
\hline AM 1054-325g ${ }^{4}$ & $\mathrm{Z3}$ & 7 & 8 & 8 & 0.08 & $2.9 \cdot 10^{7}$ & + \\
\hline AM 1054-325h ${ }^{4}$ & $\mathrm{Z} 2$ & 13 & 100 & 100 & 0.02 & $1.7 \cdot 10^{6}$ & + \\
\hline AM 1054-325j ${ }^{4}$ & $\mathrm{Z3}$ & -18 & 0 & 89 & 0.40 & $3.3 \cdot 10^{8}$ & + \\
\hline AM 1324-431d & $\mathrm{Z3}$ & 1 & 100 & 100 & 0.43 & $1.0 \cdot 10^{6}$ & - \\
\hline AM 1325-292a & $\mathrm{Z} 4$ & 22 & 8 & 8 & 0.00 & $2.4 \cdot 10^{8}$ & + \\
\hline
\end{tabular}

${ }^{1}$ For object names in boldface, velocity gradients were detected in Paper II and Weilbacher et al. (2002b).

${ }^{2} \mathrm{An}$ age with a positive sign is after, a negative sign means before the maximum star formation rate.

3' + ' denotes good S/N in the NIR, 'o' means a marginal detection, '-' is undetected.

${ }^{4}$ The properties for this object are derived using the total extinction measured in Paper II 
Second, the ages we derive for the objects of our sample are very young, the median age $^{3}$ is 1 Myr. Eight objects show ages $>3 \cdot 10^{7} \mathrm{yr}$; all these objects did either not show emission lines or had no spectra available. Because in our model these objects have already completed their starburst a long time ago, 4 of them also show high burst strengths $(b \geq 40 \%)$. A tidal tail takes about $10^{8} \mathrm{yr}$ to form, it therefore unlikely that the best fit ages $>10^{8} \mathrm{yr}$ are truly the age of the last major star formation episode in these knots. This has to be checked with a fit to a future measurement of $\mathrm{EW}(\mathrm{H} \beta)$ of the respective objects.

Most of the young objects are still within the main star formation episode or even before its maximum, and have burst strengths lower than what the best fit models reaches by the end of the starburst, if their star formation rate proceeds as predicted by the best fit model. 16 objects have young ages, between 1 and $21 \mathrm{Myr}$ after the maximum of the burst. Most of these show emission lines in their spectra, the age should therefore be well constrained. The largest group of TDG candidates actually have colors which are best fit by the models before the maximum of the burst is reached. This means they are just beginning their star formation episode. Hence, their current burst strengths are lower limits to the final value. Both values, the current burst strength and the value near the end of the burst, are shown in Cols 4 and 5 of Tab. 23.

Three objects (AM 0537-292 d, AM 0537-292 g, and AM 0748-665 a) seem to have very high burst strengths from $40 \%$ to $100 \%$ and plausible ages between 17 and $74 \mathrm{Myr}$ which indicate that they have already completed their starburst phase. At this stage in their evolution already all of the massive stars born in the starburst should have exploded as supernovae, causing a large energy output into the surrounding medium (the lifetime of stars with masses of $25 M_{o} d o t$ is $\approx 8 \mathrm{Myr}$ ). Given the fragile nature of TDGs in the gravitational field of the parent galaxy and such strong energy release, it is astonishing that these knots were not destroyed. Some other knots near the beginning of the starburst may also reach very high burst strengths, if the star formation rate continues as in the best fit models. The existence of the three aformentioned knots is therefore a great challenge to be explained by future dynamical models.

The star formation rates we derive here seem to be low as compared to those estimated directly from the $\mathrm{H} \alpha$ luminosity. However, it was shown by Weilbacher \& Fritze-von Alvensleben (2001) that $L(\mathrm{H} \alpha)$ is a poor indicator for the current SFR of extragalactic systems where the SFR changes on timescales $<10^{7} \mathrm{yr}$. In such objects, to which mainly dwarf starbursts including TDGs belong, the H $\alpha$-based estimate may underestimate the SFR by a factor of 20 or overestimate it by up to

\footnotetext{
${ }^{3}$ We derive mean and median values only for those objects, where we reached a good fit to the SED with the models from our grid, i.e. those objects in the upper part of Table 23 with the exception of AM 0748-665 d.
} 
a factor of 100 depending on the time within the burst when $\mathrm{H} \alpha$ is measured. The SFR we derive here is the first application of the method proposed by Weilbacher \& Fritze-von Alvensleben (2001) to compute a best fit model based on several filters in the optical-NIR wavelength range and the gaseous emission to derive a better estimate of the current SFR. While we can also derive an estimate for the objects where no emission line strenghts are available, this is uncertain without having an $\mathrm{EW}(\mathrm{H} \beta)$ to constrain the age within a model. The objects for which the EW was used for the analysis are marked bold in Tab. 23; for these TDG candidates the values should give a reliable estimate of the current SFR. The mean value for the 13 objects with reliable SFR estimates is $\psi=0.05 \pm 0.04 M_{\odot} \mathrm{yr}^{-1}$. If this is put into relation to the derived stellar masses and the size of the knots, it becomes clear that the TDG candidates are strongly star-forming systems. Scaled up to the mass of a spiral galaxy this hypothetical spiral would have a SFR of $>10 M_{\odot} \mathrm{yr}^{-1}$, similar to the value of the nearby starburst galaxy M 82 (de Grijs et al. 2001).

A good fit to the observed SED also enables us to determine the stellar masses of the TDG candidates by scaling the stellar mass of the model with the ratio of observedto-model luminosity. This estimate is only reliable if NIR photometry is available for the respective object with good S/N. Only stellar masses for objects marked with a ' + ' in the last column of Tab. 23 can therefore be regarded as a good estimate. The mean stellar mass for these 18 objects is $M_{\text {stellar }}=(2.4 \pm 6.3) \cdot 10^{8} M_{\odot}$. Although the given $1 \sigma$ scatter is large it seems that the stellar mass in these objects is slightly higher than expected for galaxies of their luminosity (Mateo 1998).

\subsection{TDG candidates with observed velocity gradients}

Do the knots with decoupled gas kinematics, as described in Paper II, have properties different from the other TDG candidates in the sample?

The velocity curves were derived from the brightest emission lines. As only young objects with burst ages of a few Myrs can have emission lines, the velicity gradients are all observed in young objects where the starburst is still active or ended not long ago (ages $-14 \ldots 21 \mathrm{Myrs}$ ). In other respects these object do not seem to have properties much different from all the other TDG candidates. Some have high burst strengths, whereas in some others the old stellar component dominates. The mean SFR of those 10 knots is identical to that of the whole sample $\left(\psi=0.05 \pm 0.04 M_{\odot} \mathrm{yr}^{-1}\right)$.

If we had detected velocity gradients in those objects which are the most massive TDG candidates in our sample, this should be visible also in the stellar mass. The mean stellar mass of the 6 reliably estimated masses is $M_{\text {stellar }}=(1.2 \pm 1.2) \cdot 10^{8} M_{\odot}$ which is not significantly different than the mean mass derived for the whole sample given the large spread. This supports the view presented in Paper II and Weilbacher et al. (2002b) that the large amplitudes of the velocity gradients cannot be caused by 


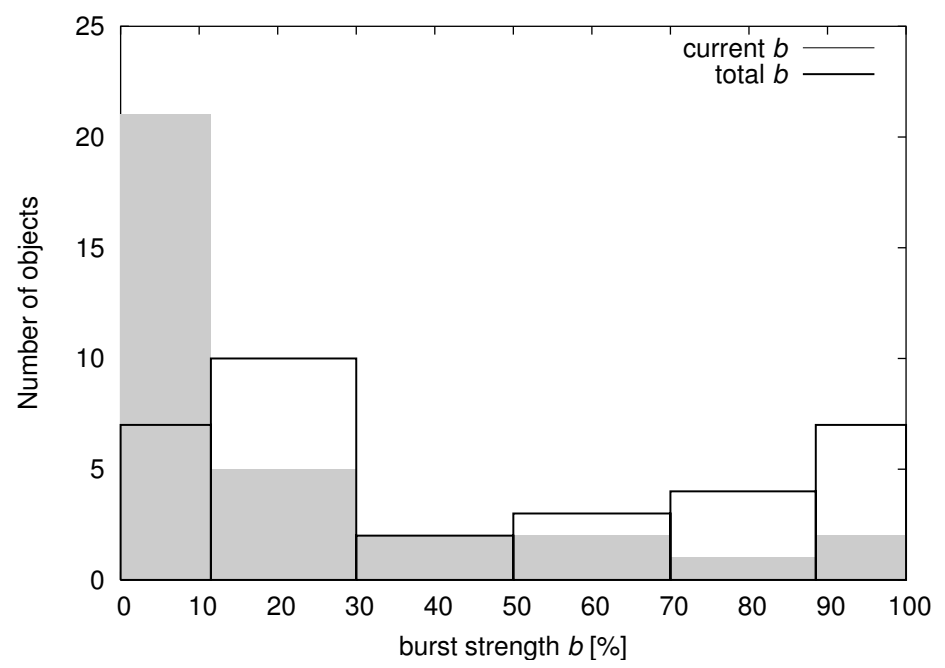

Figure 53: Comparison of the distribution of the burst strenghts at the present time (current $b$, filled boxes) and those near the end of the burst (total $b$, open boxes) for the sample of TDG candidates.

Keplerian rotation alone. If these velocity anomalies are not the result of projection effects then they may be the result of several gaseous clumps falling towards each other to form a larger conglomerate, perhaps a real TDG. Then the amplitude of the velocity gradient would be a typical free-fall velocity and not related to the stellar mass.

\subsection{TDGs: Built from gaseous or stellar clumps?}

Whether TDGs form as stellar clumps or from instabilities in the gas is one of the fundamental questions since the antithetic models of Barnes \& Hernquist (1992) and Elmegreen et al. (1993) first produced TDG-like objects. The relevant factor from our study to analyze is the burst strength $b$. While the TDG candidates studied here seem to have burst strengths much larger than the mean value of $b \approx 1 \% \mathrm{ob}$ served in BCDs (Krüger 1992), there does not seem to be a clear answer from this variable as to which mode of TDG formation is preferred by nature.

The results presented in Tab. 23 are shown graphically in Fig. 53 as a histogram for the two $b$ values (the current and the total burst strength) listed in the table. The plot shows that there is no clear dichotomy between a fraction of objects with burst strengths $>90 \%$ and another group of objects with $b<10 \%$ without anything in between these extremes. Instead, we see a continuous distribution in the ratio of the young to the old stellar population. 
From the present time until the end of the starburst the young knots will experience more star formation and their burst strengths will increase. This trend is also seen in Fig. 53, where from the current $b$ to the total $b$ the distribution is shifted to higher burst strengths. There are more objects with current and total burst strengths below $30 \%$ than above $70 \%$. At first glance this would hint to a formation from stellar clumps. The masses given by Barnes \& Hernquist (1992) are $10^{7} \ldots 10^{8} M_{\odot}$ for their stellar condensations, in the range of the stellar masses estimated here. So, our results suggest that more knots are formed from stellar clumps, while a few may be formed from condensations of the gas.

However, while we derived the stellar mass of the TDG candidates, nothing is known about their total mass which also includes atomic and molecular gas. If a knot has a small stellar mass a present burst strength of e.g. 10\%, but is associated with a gas cloud of $20 \times$ the stellar mass, it would appear as stellar clump with low content of young stars. Instead, it has a reservoir for a very strong and long lasting starburst and in the end, the total burst strength could be well above $50 \%$ given a high star formation efficiency.

The conclusion from this discussion is that in our sample more of the TDG candidates seem to be formed as stellar clumps than from gaseous condensations. This result, however, strongly depends on the time during the evolution of the knot when it is observed and is slightly different than from conclusions drawn from previous examples of TDGs in the prototype interacting galaxies NGC 7252 (Hibbard et al. 1994), Arp 105 (Duc et al. 1997), and Arp 245 (Duc et al. 2000). In those systems, the TDGs are associated with huge gas clouds which, despite a substantial mass fraction of old stars, could play an important dynamical role in the formation of TDGs.

\section{Conclusions}

We analyzed a sample of 14 southern interacting galaxies using photometry of deep NIR images obtained with the SOFI camera at the ESO NTT. In these systems, 41 candidates for TDGs have been selected previously on optical images. Using the photometric results from the optical imaging in $B, V, R$ (Paper I) and the magnitudes in up to three NIR filters presented in the present paper, we can produce an opticalNIR spectral energy distribution for each observed TDG candidate. We compute new evolutionary synthesis models for various metallicities specifically adapted to the bi-modal star formation history of TDGs, and refined with spectral synthesis and new emission line ratios. We fit the observed SED with the help of a grid of spectrophotometric models. The $\mathrm{H} \beta$ equivalent width and oxygen abundance derived from the spectroscopy (Paper II) help to restrict the age of the starburst and 
the metallicity of the model. From this comparison we derive the burst strength of the TDG candidates, their current star formation rate, and their stellar mass.

The stellar masses of the objects in our sample have a mean value of $\approx 2.4 \cdot 10^{8} M_{\odot}$, a value slightly higher than those of "normal" dwarf galaxies and in agreement with the masses derived in the models of Barnes \& Hernquist (1992). While the starburst populations in most of the TDG candidates have young ages and some also have high bursts strengths $b$, more objects are found in the lower range with $b<30 \%$, i.e. their stellar population is dominated by the "old" stellar population inherited from the parent galaxy. Although we observe a continuous range of burst strengths and the difference in number is small, it therefore seems that most of the TDG candidates in our sample are formed by mainly stellar condensations, the formation mechanism suggested by Barnes \& Hernquist (1992), and not from the collapse of giant gas clouds.

We thank K. Noeske for active help in the NIR data reduction. PMW acknowledges partial support from DFG grants FR 916/6-1 and FR 916/6-2.

\section{References}

Arp, H.C., Madore, B.F., 1987, A Catalogue of Southern Peculiar Galaxies and Associations, Cambridge University Press, Cambridge

Barnes, J.E., Hernquist, L., 1992, Nature 360, 715

de Grijs, R., O’Connell, R.W., Gallagher, J.S., 2001, AJ 121, 768

Duc, P.-A., Brinks, E., Springel, V., et al., 2000, AJ 120, 1238

Duc, P.-A., Brinks, E., Wink, J.E., Mirabel, I.F., 1997, A\&A 326, 537

Duc, P.-A., Mirabel, I.F., 1998, A\&A 333, 813

Elmegreen, B.G., Kaufman, M., Thomasson, M., 1993, ApJ 412, 90

Hibbard, J.E., Guhathakurta, P., van Gorkom, J.H., Schweizer, F., 1994, AJ 107, 67

Izotov, Y.I., Thuan, T.X., 1998, ApJ 500, 188

Izotov, Y.I., Thuan, T.X., Lipovetsky, V.A., 1994, ApJ 435, 647

Izotov, Y.I., Thuan, T.X., Lipovetsky, V.A., 1997, ApJS 108, 1 
Krüger, H., 1992, Blaue Kompakte Zwerggalaxien. Modellrechnungen zur metallizitätsabhängigen spektralen Entwicklung vom nahen Infrarot bis zum Röntgenbereich, PhD Thesis, Universität Göttingen

Krüger, H., Fritze-von Alvensleben, U., Loose, H.-H., 1995, A\&A 303, 41

Lejeune, T., Cuisinier, F., Buser, R., 1997, A\&AS 125, 229

Lejeune, T., Cuisinier, F., Buser, R., 1998, A\&AS 130, 65

Mateo, M.L., 1998, ARA\&A 36, 435

Noeske, K.G., Papaderos, P., Caíros, L.-M., Fricke, K.J., 2002, A\&A Submitted

Persson, S.E., Murphy, D.C., Krzeminski, W., Roth, M., Rieke, M.J., 1998, AJ 116, 2475

Salpeter, E.E., 1955, ApJ 121, 161

Schlegel, D.J., Finkbeiner, D.P., Davis, M., 1998, ApJ 500, 525

Stasińska, G., 1984, A\&AS 55, 15

Stasińska, G., Schaerer, D., Leitherer, C., 2001, A\&A 370, 1

Weilbacher, P.M., Duc, P.-A., Fritze-von Alvensleben, U., 2002a, $A \& A$ In press

Weilbacher, P.M., Duc, P.-A., Fritze-von Alvensleben, U., Martin, P., Fricke, K.J., 2000, A\&A 358, 819

Weilbacher, P.M., Fritze-von Alvensleben, U., 2001, A\&A 373, L9

Weilbacher, P.M., Fritze-von Alvensleben, U., 2001, in: Dwarf Galaxies and their Environment (eds. K.S. de Boer, R.-J. Dettmar, U. Klein), 287-290

Weilbacher, P.M., Fritze-von Alvensleben, U., Duc, P.-A., Fricke, K.J., 2002b, ApJ 579, L79 


\section{Paper IV}

\section{On Star Formation Rates in Dwarf Galaxies}



This paper was published July 2001 as letter in

Astronomy \& Astrophysics., 373, L9-L12.

\title{
On Star Formation Rates in Dwarf Galaxies
}

\author{
Peter M. Weilbacher, Uta Fritze-v.Alvensleben \\ Universitäts-Sternwarte, Geismarlandstr. 11, 37083 Göttingen, Germany
}

\begin{abstract}
We present evolutionary synthesis models of starbursts on top of old stellar populations to investigate in detailed time evolution the relation between $\mathrm{H} \alpha$ luminosity and star formation rate (SFR). The models show that several effects have an impact on the ratio between $L(\mathrm{H} \alpha)$ and SFR. Metallicity different from solar abundance, a time delay between star formation and maximum $\mathrm{H} \alpha$-luminosity, and a varying stellar initial mass function give rise to strong variations in the ratio of $\mathrm{H} \alpha$ luminosity to SFR and can cause large errors in the determination of the SFR when employing well-known calibrations. When studying star-bursting dwarf galaxies, and sub-galactic fragments at high redshift, which show SFR fluctuating on short timescales, these effects can add up to errors of two orders of magnitude compared with the calibrations. To accurately determine the true current SFR additional data in combination with models for the spectral energy distribution are needed.
\end{abstract}

Key words: Galaxies: starbursts - galaxies: dwarf - galaxies: evolution galaxies: fundamental parameters

\section{Introduction}

The star formation rate (SFR) is one of the basic properties of galaxies. It can be derived in different wavelength regimes (UV, optical, FIR) using empirical calibrations obtained from well studied samples of galaxy types, like spirals, irregulars, starbursts, or luminous IR galaxies. One of the most popular methods to derive SFRs from optical observations is by measuring the Balmer line fluxes, which are very sensitive to the H II regions surrounding massive young stars and therefore give a good measure of the very recent, shortlived, or ongoing SFR. When employing $\mathrm{H} \alpha$ one generally takes one of the simple linear calibrations as e.g. obtained by Hunter \& Gallagher (1986, hereafter HG86) or Kennicutt et al. (1994, KTC94) 
from observations and modeling of dwarf irregular and spiral galaxies, respectively. The calibrations are used in the form ${ }^{1}$

$$
L(\mathrm{H} \alpha)\left[\mathrm{erg} \mathrm{s}^{-1}\right]=\mathcal{V}^{2} \cdot \mathrm{SFR}\left[\mathrm{M}_{\odot} \mathrm{yr}^{-1}\right]
$$

where the $\mathcal{V}^{2}$-factor is $1.41 \cdot 10^{41}$ (HG86) or $1.26 \cdot 10^{41}$ (KTC94, Kennicutt 1998) for a Salpeter IMF in the mass range of 0.1 to $100 \mathrm{M}_{\odot}$.

These $L(\mathrm{H} \alpha)$-SFR calibrations were derived for "normal" galaxies with modest star formation rates. For systems which behave differently from the ones for which the calibrations were derived, the application of this method may not be appropriate. The validity of the SFR-determination in star-bursting galaxies, like e.g. blue compact dwarfs (BCDs), where these relations are frequently used, has never been shown.

In fact, as the strongest output of ionizing photons is related to the most luminous, i.e. giant or supergiant phases of the ionizing stars, there may be a small time delay between abrupt changes in the SFR and the corresponding changes in the $\mathrm{H} \alpha$ flux. Leitherer et al. (1995) showed that such a time dependence exists for stellar features in the UV. For systems with SF fluctuations on short timescales, the delay effect of the Balmer lines can also be important, e.g. for small scale systems like dwarf galaxies, where star burst durations are usually assumed to be of the order of a dynamical timescale, i.e. $10^{5}$ to $10^{6}$ years. SFR fluctuating strongly on short timescales may also have taken place in sub-galactic fragments before merging together to hierarchically build up today's galaxies (Glazebrook et al. 1999).

We will show that indeed one may make very large errors when blindly applying the usual calibrations to small systems. We first present details on our model in Sect. 2. We then describe different effects that can affect the calibrations for $L(\mathrm{H} \alpha)$ in terms of SFR, namely metallicity (Sect. 3), short burst durations (Sect. 4), and changes in the IMF (Sect. 5). We finally summarize our results in Sect. 6.

\section{Model description}

We use our evolutionary synthesis code specifically adapted to the modeling of starbursts in dwarf galaxies (Krüger et al. 1995, Weilbacher et al. 2000). It includes specific modeling of gaseous emission lines and continuum based on the Lyman continuum photons emitted by hot young stars.

We use the current Geneva stellar tracks (see Lejeune \& Schaerer 2001, for a recent compilation) in the metallicity range from $Z_{\odot} / 20$ to $2 Z_{\odot}$. To be compatible with HG86 and KTC94 we use the Salpeter IMF (Salpeter 1955) in the range of 0.15 to

\footnotetext{
${ }^{1}$ The name of the variable $\mathcal{V}^{2}=L(\mathrm{H} \alpha) /$ SFR is deduced from the fact, that the unit of $\mathcal{V}^{2}$ actually is a squared velocity.
} 
85 or $120 \mathrm{M}_{\odot}$, as given by the tracks. We use the recently revised Lyman continuum photon emission rates as given by Schaerer \& de Koter (1997), who account for nonLTE effects, line blanketing, stellar winds, and the new temperature and gravity calibrations by Vacca et al. (1996). To derive the H $\alpha$ luminosity, we sum up the emerging Lyman continuum photon emission $N\left(\mathrm{H}^{0}\right)$ for each star, and convert it to a luminosity in $\mathrm{H} \alpha$ using

$$
L(\mathrm{H} \alpha)\left[\mathrm{erg} \mathrm{s}^{-1}\right]=1.36 \cdot 10^{-12} N\left(\mathrm{H}^{0}\right)\left[\mathrm{s}^{-1}\right] .
$$

We have updated our models using the emission line ratios for low metallicities observed by Izotov et al. (1997) and Izotov \& Thuan (1998) for a large sample of blue compact dwarfs (BCDs).

We present two types of one-zone models. The first represents a quiescent galaxy, where the SFR decreases slowly from the formation epoch with a timescale of 10 Gyrs. The other model was already discussed in detail by Weilbacher et al. (2000) in their interpretation of Tidal Dwarf Galaxy candidates, and can also be used to model blue compact dwarfs (BCDs, see Krüger et al. 1995). Here we put a starburst on top of the stellar population of the undisturbed model. It is assumed to reach its maximum SFR of $20 \mathrm{M}_{\odot} \mathrm{yr}^{-1}$ after $10 \mathrm{Gyrs}$, and we vary the burst timescale $\tau_{\mathrm{B}}$ from $10^{5}$ to $10^{8} \mathrm{yrs}$ to model all the range of burst durations from small dwarf galaxies to mergers of giant gas-rich galaxies. We alternatively a bell shaped (Gaussian) burst or sharply rising and exponentially decreasing starburst. We assume a minimum SFR after the starburst of $0.1 \mathrm{M}_{\odot} \mathrm{yr}^{-1}$.

\section{Effects of Metallicity}

We successfully reproduce the calibrations of HG86 and KTC94 using our quiescent models using solar metallicity and upper mass limits of 120 and $85 \mathrm{M}_{\odot}$, respectively. The values we derive for the four other metallicities are given in Tab. 24. While for solar metallicity models the agreement with HG86's and KTC94's empirical calibrations is very good, it is also seen in Tab. 24 that both towards lower and higher metallicities the differences become significant. For subsolar metallicities as e.g. in $\mathrm{BCDs}\left(\langle Z\rangle_{\mathrm{BCD}}=0.002 \pm 0.001=1 / 10 Z_{\odot}\right.$, Izotov \& Thuan 1998) the difference in $\mathcal{V}^{2}$ is found to be a factor of $\sim 3.5$. This is a result of low metallicity stellar populations being both more luminous and hotter than a solar metallicity stellar population with the same IMF and mass limits.

Application of the empirical calibrations to estimate SFRs from H $\alpha$ luminosities in low metallicity dwarf galaxies hence can yield SFRs overestimated by a factor $\gtrsim 3$ due to metallicity effects. We also give in Tab. 24 the $\mathcal{V}^{2}$-value obtained in our solar metallicity model using the IMF of Scalo (1986) instead of Salpeter's. Due to the 
Table 24: Calibrations for different metallicities $Z$.

\begin{tabular}{lr|cc}
$Z$ & $M_{\text {up }}$ & $\mathcal{V}^{2}$ & $\mathcal{V}_{[\mathrm{O} \mathrm{II}]}^{2}$ \\
& {$\left[\mathrm{M}_{\odot}\right]$} & {$\left[10^{41} \frac{\mathrm{ergs}^{-1}}{\mathrm{M}_{\odot} \mathrm{yr}^{2}}\right]$} \\
\hline 0.001 & 85 & 4.221 & 1.923 \\
0.001 & 120 & 4.702 & 2.142 \\
0.004 & 85 & 3.105 & 1.944 \\
0.004 & 120 & 3.315 & 2.076 \\
0.008 & 85 & 2.073 & 2.182 \\
0.008 & 120 & 2.548 & 2.682 \\
0.020 & 85 & 1.229 & 1.293 \\
0.020 & 120 & 1.408 & 1.482 \\
0.040 & 85 & 0.902 & 0.949 \\
0.040 & 120 & 0.997 & 1.049 \\
\hline 0.020 (S86) & 120 & 0.917 & 0.965 \\
HG86 & 100 & 1.41 & - \\
KTC94 & 100 & 1.26 & -
\end{tabular}

smaller number of high mass stars in case of a Scalo-IMF, the same SFR produces an $\mathrm{H} \alpha$ luminosity that is lower by $35 \%$.

All other hydrogen lines, as e.g. $\mathrm{L}_{\alpha}$ or $\mathrm{Br}_{\gamma}$, which can also be used to estimate SFRs, and their respective calibration factors can easily be computed from our values for $\mathrm{H} \alpha$ and Eq. (3).

At higher redshift, the [O II] 3727 line is frequently used to derive SFRs, as e.g. for Lyman Break galaxies. This line involves a direct metallicity dependence in addition to the one inherent in the hydrogen lines which is due to differences in temperature and luminosity of stellar populations at various metallicities. Since high redshift star-forming objects and, in particular, sub-galactic fragments both have low metallicities and possibly strongly fluctuating SFRs, care is needed to derive SFRs from $L\left(\left[\mathrm{OII}^{\mathrm{II}}\right]\right)$. We present the values of the $\mathcal{V}_{[\mathrm{OII}]}^{2}$-factor in Col. 4 of Tab. 24 for the [O II] 3727 line, where the according linear relation with SFR is

$$
L([\mathrm{OII}])\left[\mathrm{erg} \mathrm{s}^{-1}\right]=\mathcal{V}_{[\mathrm{OII}]}^{2} \cdot \mathrm{SFR}\left[\mathrm{M}_{\odot} \mathrm{yr}^{-1}\right] .
$$

\section{Short starbursts}

In Fig. 54 we show the time evolution of $L(\mathrm{H} \alpha)$ for rapidly rising (instantaneous) starbursts with short timescales $\left(\tau_{\mathrm{B}}=10^{6} \mathrm{yr}\right.$ ) for five metallicities. All models have their maximum SFR at a time of 10.0 Gyrs. We note two effects: For lower metallicity $Z$ the maximum $\mathrm{H} \alpha$ luminosity is higher than for high metallicity, e.g. by 


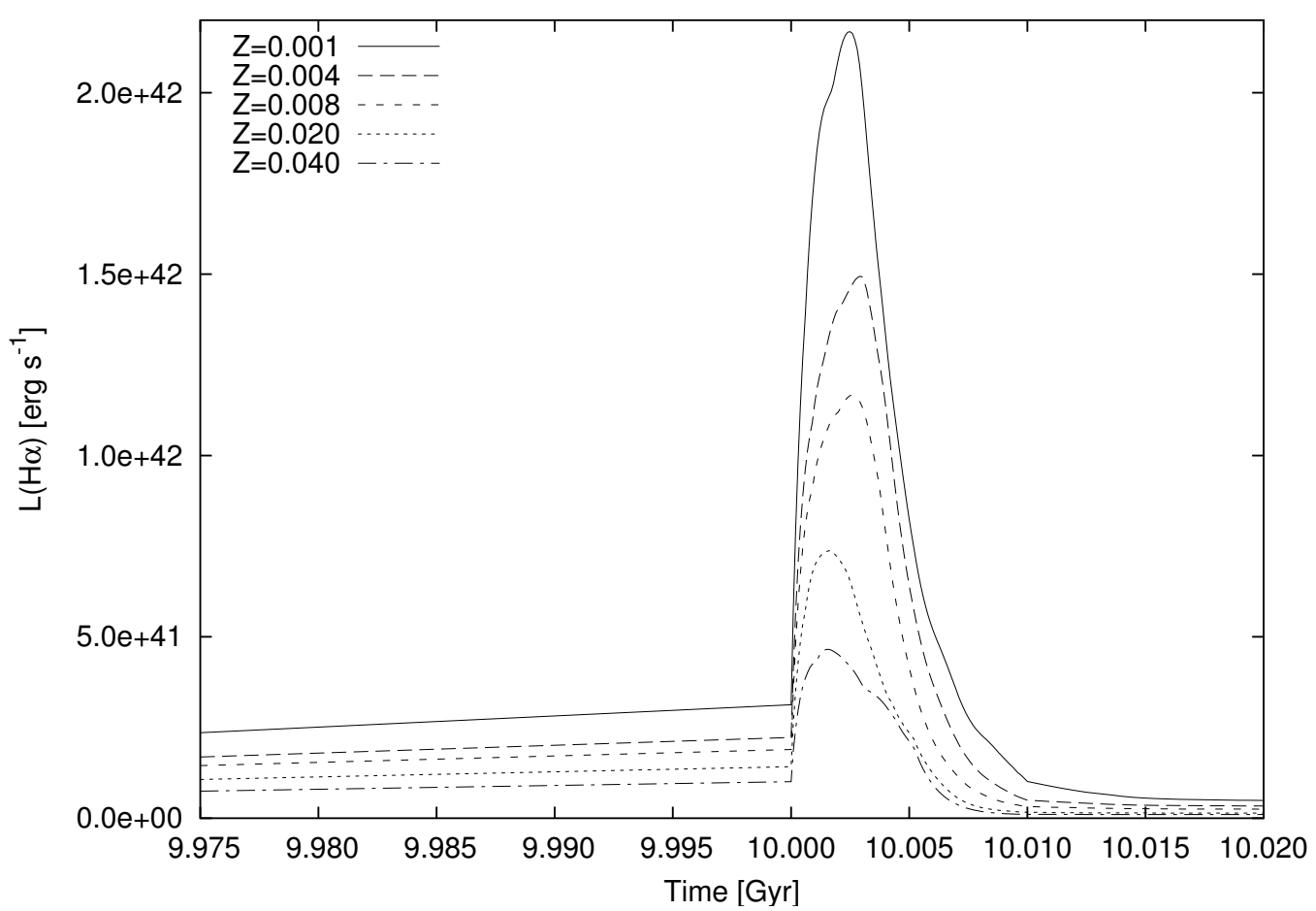

Figure 54: $L(\mathrm{H} \alpha)$ over time for instantaneous starbursts with short timescale of $\tau_{\mathrm{B}}=10^{6} \mathrm{yr}$.

a factor of 4.6 in case of $Z=0.001$ as compared with $Z=0.040$. This is due to the higher temperatures of the low metallicity stars, which more efficiently ionize the interstellar medium. It is also apparent that there is a delay of the maximum in the H $\alpha$ luminosity with regard to the maximum of the SFR. The offset between maximum SFR and maximum $L(\mathrm{H} \alpha)$ is higher for lower metallicities, 2.9 Myrs for $Z=0.004$ vs. $1.5 \mathrm{Myrs}$ for $Z=0.040$. This again is a result of the higher temperatures and hence the ionizing power of low metallicity stars. At low metallicity stars of lower mass and longer main sequence lifetimes contribute to the Lyman continuum emission. Since those take longer to reach their maximum Lyman continuum emission rates during their supergiant phase the delay becomes slightly longer than in the solar metallicity case.

In Fig. 55 we plot the ratio between $L(\mathrm{H} \alpha)$ and the SFR of our models as a function of time for Gauss-shaped bursts with a short timescale of $\tau_{\mathrm{B}}=10^{6} \mathrm{yr}$. Before and after the starburst the empirical linear calibrations agree well with the ratio seen in our solar metallicity model. With the onset of the burst the SFR rises faster than $L(\mathrm{H} \alpha)$ due to the delay in the maximum Lyman continuum photon production shown before in Fig. 54. Therefore the $\mathcal{V}^{2}$-factor first decreases. After its minimum $\mathcal{V}^{2}$ increases, because $L(\mathrm{H} \alpha)$ continues to rise until the ionizing stars have reached 


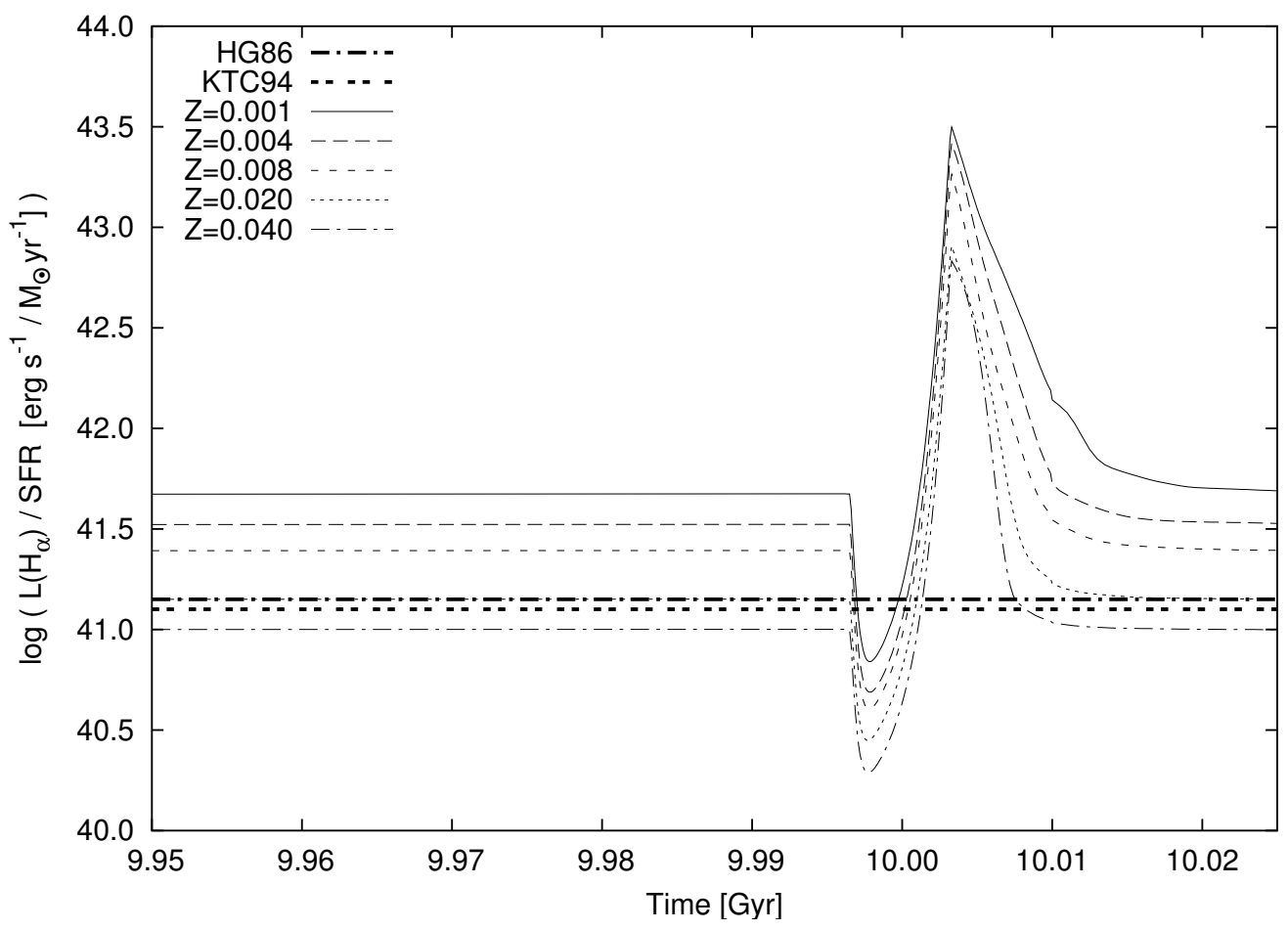

Figure 55: $\mathcal{V}^{2}=L(\mathrm{H} \alpha)$ SFR over time for Gaussian bursts with short timescale of $\tau_{\mathrm{B}}=10^{6} \mathrm{yr}$.

their supergiant phase. When the number of Lyman continuum photons and therefore $L(\mathrm{H} \alpha)$ become maximal, the SFR has already decreased from its maximum value by a factor of $\sim 3$. After its maximum $L(\mathrm{H} \alpha)$ declines as the death rate of $\mathrm{O}$ stars is no longer compensated by SF. In this phase, however, the SFR decreases even faster than the H $\alpha$ flux, and $\mathcal{V}^{2}$ continues to increase. The sharp peak visible near 10.003 Gyrs, after which $\mathcal{V}^{2}$ abruptly decreases, is a result of the constant minimum SFR of our models after the burst. If the SFR after the burst would go to zero, $\mathcal{V}^{2}$ would diverge. The minimum SFR, which starts near 10.003 Gyrs, then acts to slowly bring down $\mathcal{V}^{2}$ to values in agreement with those in Tab. 24. This time sequence during the starburst and the interplay between $L(\mathrm{H} \alpha)$ and SFR can also be seen in Fig. 57 below.

It is obvious from Fig. 55 that for bursts with $\tau_{\mathrm{B}}=10^{6} \mathrm{yr}$ strong discrepancies from the calibrations are seen for all metallicities, strongest at the lowest metal abundance, amounting to a difference of nearly two orders of magnitude as compared to the calibrations, even for solar metallicity models.

In Fig. 56 we show the evolution of the $\mathcal{V}^{2}$-factor with time for five Gaussian starburst models with different burst timescales for our lowest metallicity $Z=0.001$. With increasing burst duration, the maximum is shifted towards later times. It is 


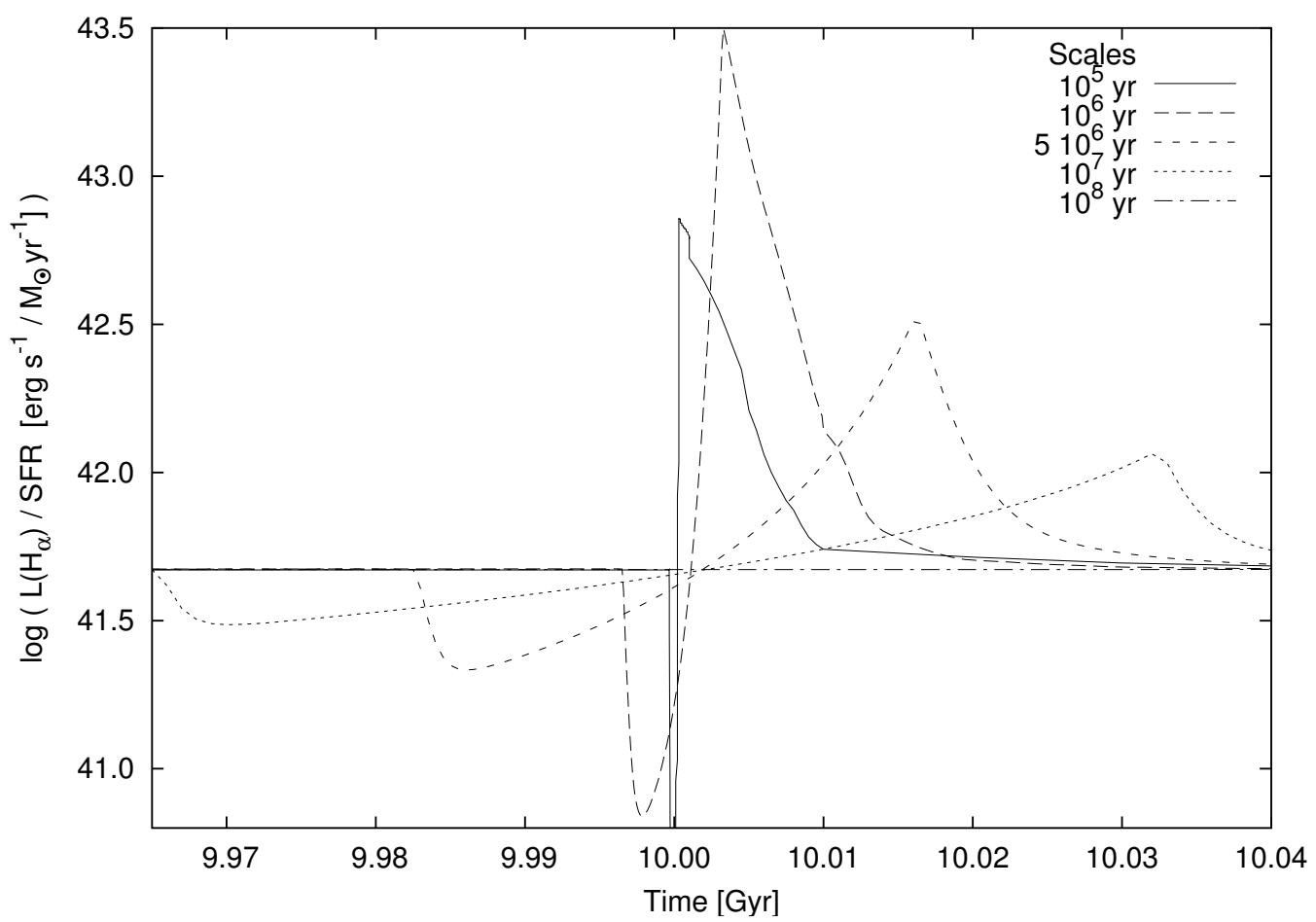

Figure 56: $\mathcal{V}^{2}=L(\mathrm{H} \alpha) /$ SFR over time for Gaussian bursts with different timescales and $Z=0.001$.

strongest for bursts with $\tau_{\mathrm{B}}=10^{6} \mathrm{yr}$ (which are shown in Fig. 55). This is a result of the convolution of the Gauss-shaped increase of the burst-SFR with the delay in maximum $\mathrm{H} \alpha$ emission due to the most massive and most luminous (= giant) stars. The decline after the sharply peaked maximum is an effect of the onset of the minimum SFR as discussed above for Fig. 55. The time evolution of $\mathcal{V}^{2}$ significantly depends on the burst duration. For the longest burst with $\tau_{\mathrm{B}}=10^{8} \mathrm{yr}$ the slow change in the SFR of the burst causes the delay effect to have negligible impact on $\mathcal{V}^{2}$.

In conclusion we have shown that the SFR derived from $\mathrm{H} \alpha$ during or shortly after short bursts can be wrong by factors $\gg 10$. Averaged over the entire burst duration, however, values for $\mathcal{V}^{2}$ agree well with those from Tab. 24 for different metallicities. This applies to statistical analyses of samples of $\mathrm{H}$ II galaxies.

\section{Effects of the IMF}

Kennicutt et al. (1994) have already investigated different $\mathcal{V}^{2}$-factors with different IMFs with identical mass cutoffs and found differences of an order of magnitude 
between IMFs they used. Leitherer \& Heckman (1995) also presented properties like number of $\mathrm{O}$ stars, $N\left(\mathrm{H}^{0}\right)$, and $\mathrm{EW}(\mathrm{H} \alpha)$ etc. for instantaneous and continuous SF models with different IMFs. Here we want to show the effect of various IMFs on the time delay discussed above.

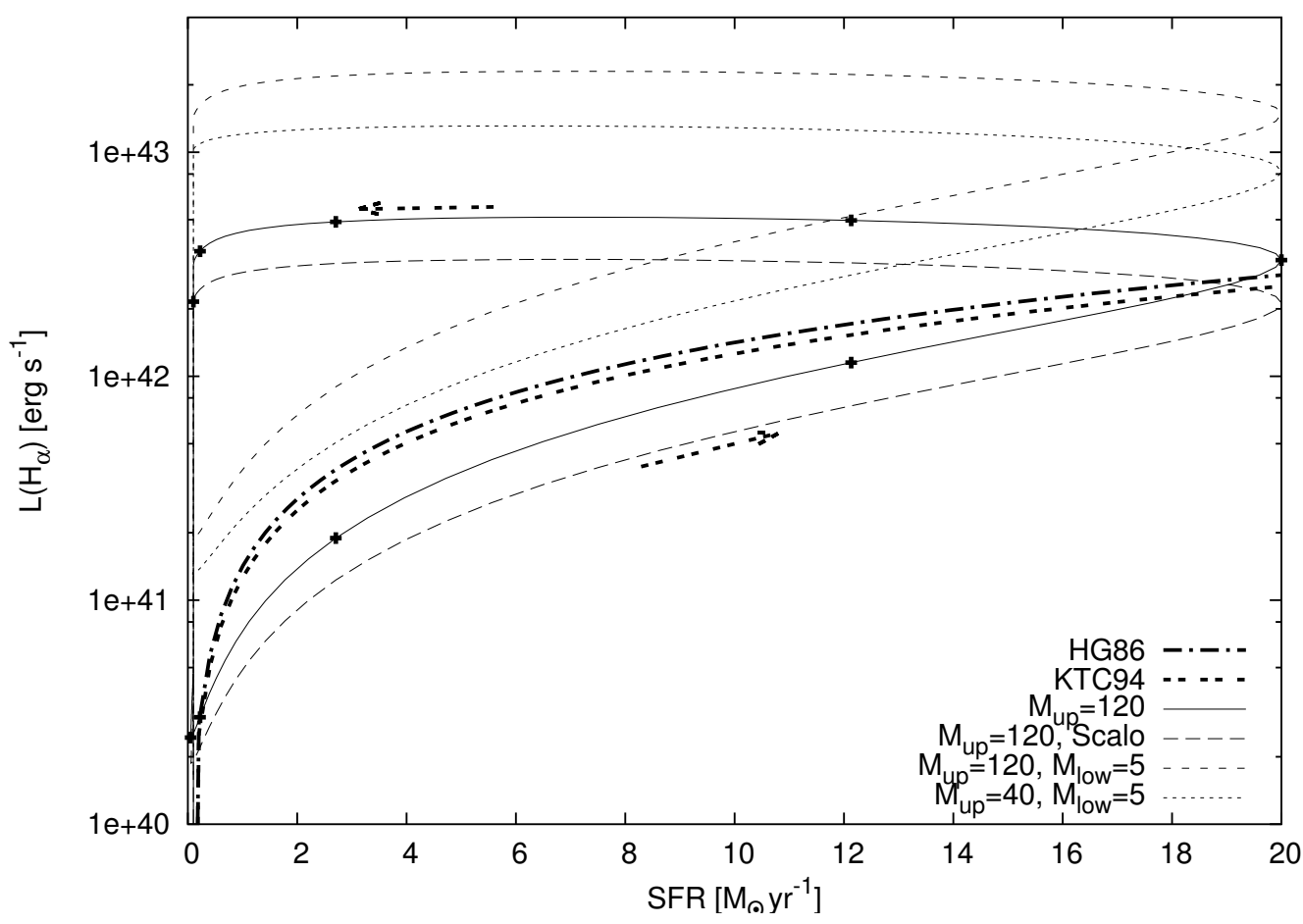

Figure 57: $L(\mathrm{H} \alpha)$ over SFR for a bursting dwarf galaxy with metallicity $Z=0.001$ and different IMF cuts.

Fig. 57 shows the luminosity $L(\mathrm{H} \alpha)$ plotted over the SFR of the model galaxy, while it experiences its starburst with a timescale of $\tau_{B}=10^{6} \mathrm{yr}$. The calibrations of HG86 and KTC94 are plotted for reference. To indicate the evolution in time our "standard model" with $\mathrm{M}_{\text {up }}=120 \mathrm{M}_{\odot}$ has additional dots each $10^{6} \mathrm{yrs}$ during the burst; the arrows show the direction of the loops in this diagram.

It is obvious that the calibrations do not represent the real SFR very well. $L(\mathrm{H} \alpha)$ at the peak SFR does fit very well with the calibrations for both models with Salpeter and Scalo IMF and a high mass cut at $\mathrm{M}_{\mathrm{up}}=120 \mathrm{M}_{\odot}$. But generally our models show that for an observed value of $L(\mathrm{H} \alpha)$ there is an ambiguity between two values of the SFR. E.g. for $L(\mathrm{H} \alpha)=10^{42} \mathrm{erg} \mathrm{s}^{-1}$ the calibrations give a SFR of $7 \ldots 8 \mathrm{M}_{\odot} \mathrm{yr}^{-1}$, while one has to choose between SFRs of $\sim 0$ and $\sim 15 \mathrm{M}_{\odot} \mathrm{yr}^{-1}$ from our model with Salpeter IMF, and one needs additional data to determine the true current SFR. 
The evolution of the models after the starburst shows that for low SFRs (lower as one would expect from the calibrations) a galaxy could show high $\mathrm{H} \alpha$ luminosity for quite some time $\left(\sim 3 \cdot 10^{6} \mathrm{yr}\right)$. For the two models with Salpeter IMF and lower mass cuts at $\mathrm{M}_{\text {low }}=5 \mathrm{M}_{\odot}$ this effect is even more extreme. $L(\mathrm{H} \alpha)$ at the peak SFR is already underestimated by the calibrations by factors of 3 and 5, respectively, for high mass cutoffs of $\mathrm{M}_{\mathrm{up}}=40$ and $120 \mathrm{M}_{\odot}$. For a given $\mathrm{H} \alpha$-luminosity the calibrations yield a SFR too high by at least one order of magnitude when compared to the values from our models.

\section{Conclusions}

When observing small scale star-forming entities like dwarf galaxies or sub-galactic fragments, where dynamical timescales and hence burst durations may typically be of the order of $10^{6} \mathrm{yrs}$ or less, one should be aware that the currently used calibrations on the basis of $\mathrm{H} \alpha$ luminosities may yield SFRs with large errors. These calibrations were determined from larger low-level star-forming systems (where they work very well), and when applying them to star-bursting dwarf galaxies, errors of factors 3 to 100 may affect this determination of the SFR. The ratio of H $\alpha-$ luminosity to SFR depends on the metallicity of the object, and the age and the duration of the starburst.

To more accurately determine the true current SFR of a (star-bursting) dwarf galaxy additional information about the spectral energy distribution (SED) is needed. Observations in at least three optical/NIR filters or a spectrum with sufficient wavelength coverage to determine the slope of the SED could be used in comparison with models that include gaseous emission to eliminate ambiguities in the relation of the H $\alpha$ luminosity to the SFR.

We thank our anonymous referee for a very prompt and helpful report. PMW is partially supported by Deutsche Forschungsgemeinschaft (DFG Grant FR 916/6-1).

\section{References}

Glazebrook, K., Blake, C., Economou, F., Lilly, S., Colless, M., 1999, MNRAS 306, 843

Hunter, D.A., Gallagher, J.S., 1986, PASP 98, 5

Izotov, Y.I., Thuan, T.X., 1998, ApJ 500, 188

Izotov, Y.I., Thuan, T.X., Lipovetsky, V.A., 1997, ApJS 108, 1 
Kennicutt, R.C., 1998, ARA\&A 36, 189

Kennicutt, R.C., Tamblyn, P., Congdon, C.E., 1994, ApJ 435, 22

Krüger, H., Fritze-von Alvensleben, U., Loose, H.-H., 1995, A\&A 303, 41

Leitherer, C., Heckman, T.M., 1995, ApJS 96, 9

Leitherer, C., Robert, C., Heckman, T.M., 1995, ApJS 99, 173

Lejeune, T., Schaerer, D., 2001, A\&A 366, 538

Salpeter, E.E., 1955, ApJ 121, 161

Scalo, J.M., 1986, Fund. Cosmic Phys. 11, 1

Schaerer, D., de Koter, A., 1997, A\&A 322, 598

Vacca, W.D., Garmany, C.D., Shull, J.M̃., 1996, ApJ 460, 914

Weilbacher, P.M., Duc, P.-A., Fritze-von Alvensleben, U., Martin, P., Fricke, K.J., 2000, A\&A 358, 819 


\section{Paper V}

\section{A Spectacular Example: AM 1353-272}



This paper was published October 2002 as letter in

Astrophysical Journal, 579, L79-L82.

\title{
Large Velocity Gradients in the Tidal Tails of the Interacting Galaxy AM 1353-272 ("The Dentist's Chair") ${ }^{1}$
}

\author{
Peter M. Weilbacher ${ }^{2}$, Uta Fritze-v. Alvensleben ${ }^{2}$, Pierre-Alain Duc ${ }^{3}$, \\ Klaus J. Fricke ${ }^{2}$
}

\begin{abstract}
We present VLT observations of the interacting system AM 1353-272. Using the FORS2 instrument, we studied the kinematics of the ionized gas along its prominent tidal tails and discovered strikingly large velocity gradients associated with seven luminous tidal knots. These kinematical structures cannot be caused by streaming motion and most likely do not result from projection effects. More probably, instabilities in the tidal tails have lead to the formation of kinematically decoupled objects which could be the progenitors of selfgravitating Tidal Dwarf Galaxies.
\end{abstract}

Key words: Galaxies: interactions - Galaxies: formation - Galaxies: kinematics and dynamics - Galaxies: individual (AM 1353-272)

\section{Introduction}

Interactions among disk galaxies are observed to produce tidal tails of sometimes impressive lengths containing stars and $\mathrm{HI}$ in varying proportions. While tidal tails of interacting galaxies have been analyzed in dynamical simulations of interactions to assess their use to probe the form of the dark matter potential (e.g. Springel \& White 1999), the detailed kinematics of structures within the tidal tails have not received much attention. Both in deep spectroscopic observations (Duc et al. 2000) and high resolution dynamical simulations (Barnes \& Hernquist 1992), condensations are observed to form in tidal tails, the nature and fate of which are not yet fully understood.

\footnotetext{
${ }^{1}$ Based on observations collected at the European Southern Observatory, La Silla, Chile (ESO No 66.B-0055).

${ }^{2}$ Universitäts-Sternwarte, Geismarlandstraße 11, 37083 Göttingen, Germany, \{weilbach, ufritze\} @ uni-sw.gwdg. de

${ }^{3}$ CNRS URA 2052 and CEA, DSM, DAPNIA, Service d'Astrophysique, Centre d'Etudes de Saclay, 91191 Gif-sur-Yvette Cedex, France, paduc@cea.fr
} 
At a distance of $159 \mathrm{Mpc}$ (computed with $H_{0}=75 \mathrm{~km} \mathrm{~s}^{-1} \mathrm{Mpc}^{-1}$ ) the interacting system AM 1353-272, nicknamed the "The Dentist's Chair" for its peculiar morphology, consists of three apparent components (see Fig. 58): 'A', a disturbed galaxy with two $\sim 40 \mathrm{kpc}$ long tidal tails (also cataloged as ESO 510-G 020G and IRAS F13533-2721), a disturbed edge-on disk galaxy ' $B$ ', and an elliptical ' $C$ ' (see Weilbacher et al. 2000). While 'A' and 'B' are a physical pair with a central velocity difference of only $\sim 150 \mathrm{~km} \mathrm{~s}^{-1}$, ' $\mathrm{C}$ ' has a heliocentric velocity of $14750 \mathrm{~km} \mathrm{~s}^{-1}$, and is therefore located $38 \mathrm{Mpc}$ behind the interacting pair. The tidal tails of AM 1353-272 A host several blue knots with luminosities of dwarf galaxies. From their location and colors, they were identified by Weilbacher et al. (2000) as Tidal Dwarf Galaxy (TDG) candidates.

In this letter, we present the first evidence from deep optical spectroscopy with the VLT that some dense structures in the tidal tails are kinematically decoupled from the overall motion of the tails. Forthcoming instruments on large telescopes - e.g. high resolution integral field spectrographs - will be very well suited to investigate further the specific dynamics in the tidal tails of AM 1353-272 A.

\section{Observations and data reduction}

We have obtained multi-object spectroscopic data of a field centered on the system AM 1353-272 with the FORS2 instrument at the 4th VLT telescope "Yepun". Service mode observations were carried out in the night 13/14th of August 2001 under photometric conditions. Several spectroscopic standards were observed. The total exposure time of $3210 \mathrm{~s}$ was split into three individual exposures of $1070 \mathrm{~s}$ to ease cosmic ray cleaning. The seeing of $1{ }^{\prime \prime} 0$ is well sampled by an instrumental scale of $0 . \prime 2 \mathrm{px}^{-1}$. The grism $600 \mathrm{~B}+22$ was used, giving a wavelength coverage of 3450 .. $5900 \AA$ and a spectral resolution of $5.7 \AA$ (1.2 $\AA$ per pixel) for a central slit of $1^{\prime \prime}$ width. We created a mask for the mask exchange unit (MXU) of the FORS2 instrument with curved and tilted slits to cover the tails of AM 1353-272 A and several surrounding galaxies in one setup (see Fig. 58).

The data reduction followed standard recipes, for which we used our own IRAF task $\operatorname{mosx}$ (Weilbacher et al. 2002). Special care was taken to correct the curvature of the 2D spectra of the curved slits: a proper wavelength calibration was carried out at each position along the slits, using a reference $\mathrm{HeHgCd}$ frame observed through the same mask as the science frames. The typical accuracy for each column is $0.04 \AA$ RMS. A 4th order polynomial fit was then performed for each slit to determine the final wavelength solution and compute the parameters of the curvature correction. All these tasks were performed using the standard tools in the IRAF package longslit. The result of this procedure is presented in Fig. 59: the original, uncorrected $2 \mathrm{D}$ spectrum is compared with the spectrum after the correction. 


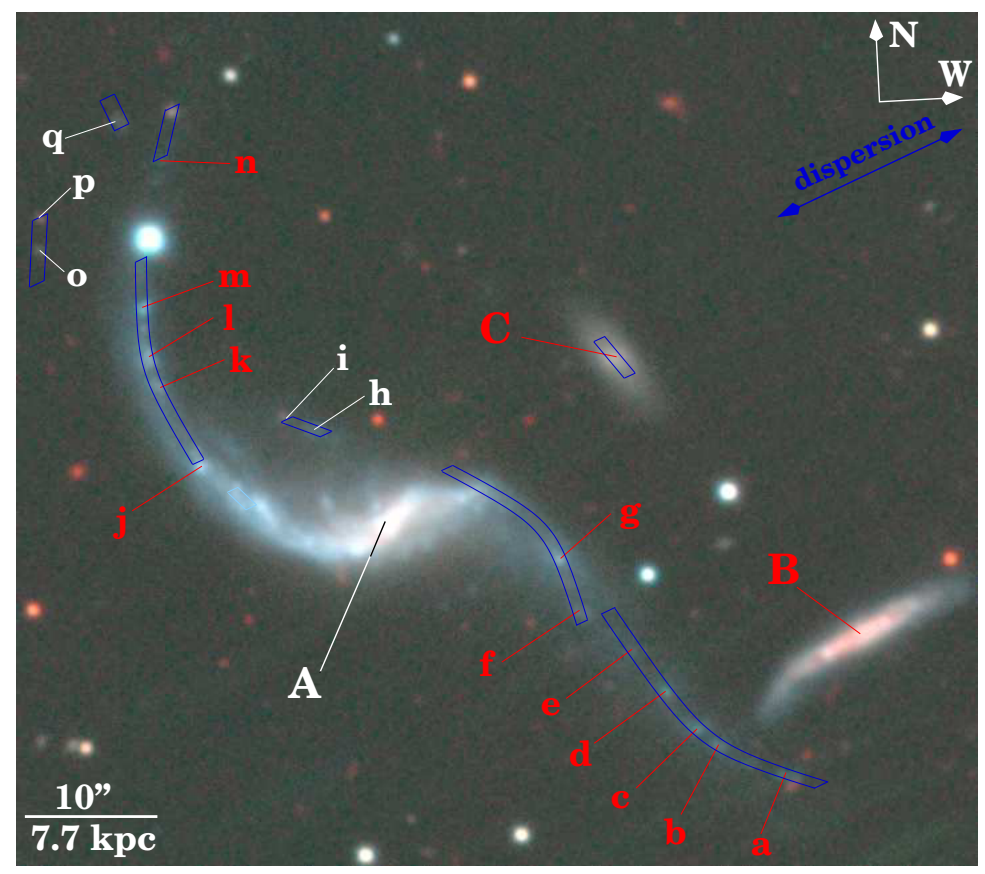

Figure 58: Finding chart of AM 1353-272. Composite $B+V+\mathrm{NIR}$ "true" color image from NTT-SUSI and SOFI (Weilbacher et al. 2000, Weilbacher et al. in prep.). The field of view is $\sim 2^{\prime} \times 2^{\prime}$. Red labels mark objects with measured redshift.

After subtracting the sky background, we used the brightest emission lines from the slits along the tidal tails to derive the velocity profiles with an IRAF script based on the fitprofs procedure. As a check, the same velocity fit was carried out with the nearest lines in the wavelength calibration spectrum. The $1 \sigma$ differences from zero velocity in this control fit are below $1 \mathrm{~km} \mathrm{~s}^{-1}$. The relative systematic errors in the final velocity profiles should be of the order of $1 \mathrm{~km} \mathrm{~s}^{-1}$ while the errors for single points are typically $15 \mathrm{~km} \mathrm{~s}^{-1}$.

\section{Results}

Fig. 60 presents the velocity distribution of the ionized gas along the two tails of AM 1353-272. The velocities indicated on the top of Fig. 60 are relative to the interpolated central velocity $V_{A}=11935 \mathrm{~km} \mathrm{~s}^{-1}$ of AM $1353-272 \mathrm{~A}$. The relative $\mathrm{H} \beta$ flux and hence distribution of the $\mathrm{H}$ II regions along the tails, is shown below: it may be used to infer the $\mathrm{S} / \mathrm{N}$ ratio of the emission lines used to derive the velocities. Vertical lines mark the individual optical knots studied by Weilbacher et al. (2000). 


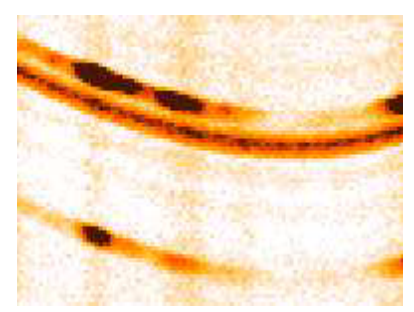

(a) original

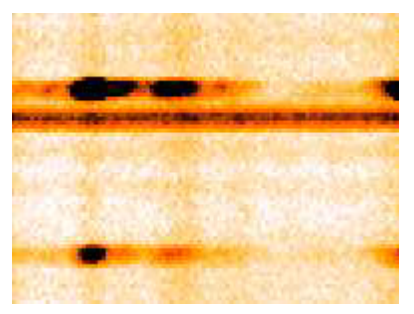

(b) corrected

Figure 59: Slit curvature correction. As an example, we show part of a $2 \mathrm{D}$ spectrum with the emission lines [O III] 5007,4959 emitted by the knots ' $\mathrm{j}$ ' to ' $\mathrm{m}$ '. Between these object lines, a skyline is visible which allows to judge the quality of the correction.
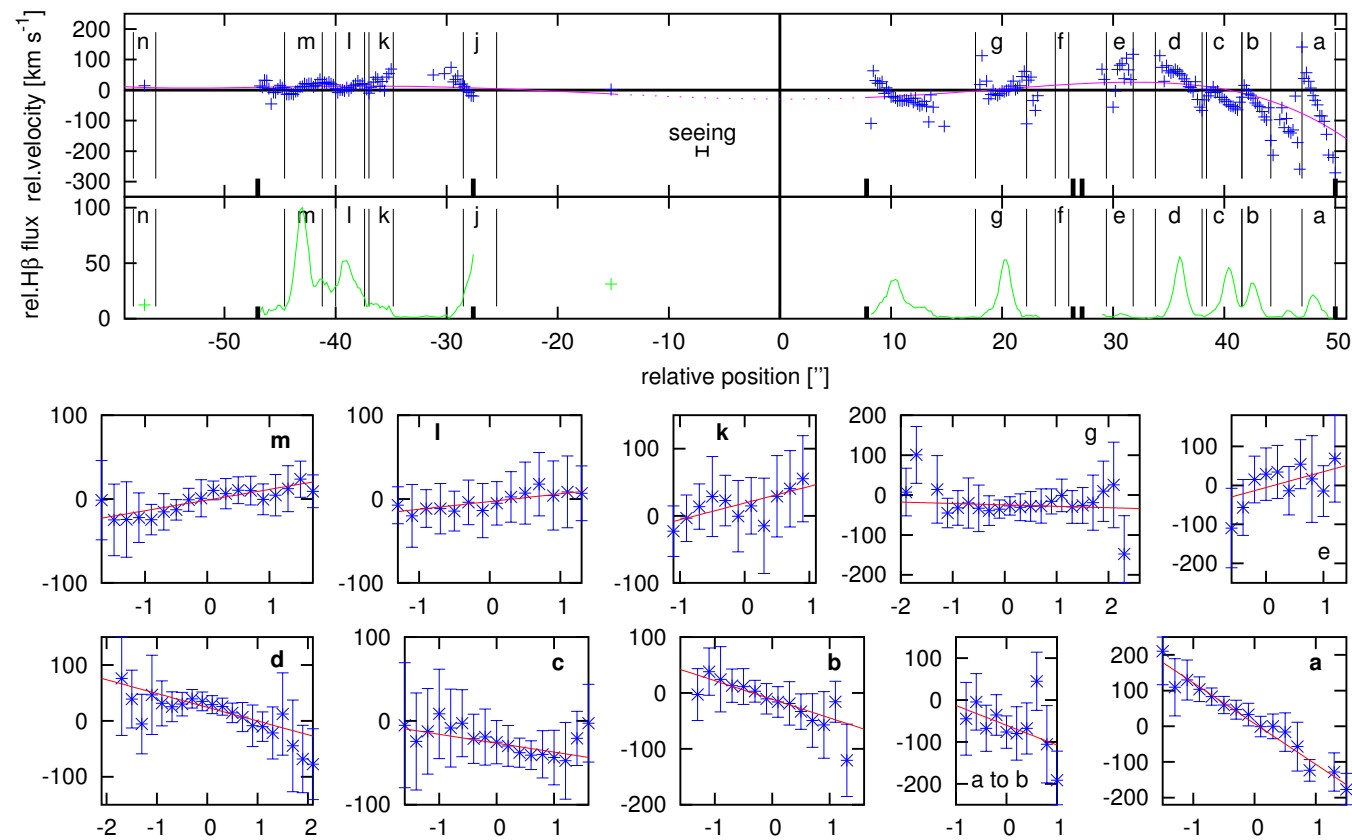

Figure 60: Velocities along AM 1353-272 A. Top: velocity field relative to a zeropoint of $11935 \mathrm{~km} \mathrm{~s}^{-1}$ along the ridge of the galaxy from the northern (left) to the southwestern (right) tip of the tidal tail. The seeing of 1 ."$^{\prime \prime} 0$ is indicated. The lower part shows the $\mathrm{H} \beta$ flux in relative units on the same spatial scale. The size of each knot is marked with vertical lines. Bold marks on the position axis indicate the ends of the curved slits. A 5th order fit to the overall velocity profile is shown. Bottom: the extracted residual velocity field of each knot after correction for tidal motion, plotted as relative position $\left[{ }^{\prime \prime}\right]$ vs. relative velocity $\left[\mathrm{km} \mathrm{s}^{-1}\right]$. Lines show a linear fit. 
Table 25: Velocity gradients within the knots.

\begin{tabular}{|c|c|c|c|}
\hline $\mathrm{ID}^{\mathrm{a}}$ & $\begin{array}{c}\Delta V_{\max }^{\mathrm{b}} \\
{\left[\mathrm{km} \mathrm{s}^{-1}\right]}\end{array}$ & error & $\begin{array}{l}\text { extent }^{\mathrm{c}} \\
{\left[{ }^{\prime \prime}\right],[\mathrm{kpc}]}\end{array}$ \\
\hline $\mathbf{a}$ & +343 & $5 \%$ & $3.0,2.3$ \\
\hline$a-t o-b^{d}$ & +94 & $72 \%$ & $2 !{ }^{\prime \prime} 0,1.5$ \\
\hline b & +87 & $21 \%$ & $2 !{ }^{\prime \prime} 6,2.0$ \\
\hline c & +34 & $40 \%$ & $3 !$ !' $2,2.5$ \\
\hline d & +93 & $10 \%$ & $4 . " 2,2.9$ \\
\hline e & -73 & $65 \%$ & $2 ! " 4,1.4$ \\
\hline $\mathrm{f}$ & \multicolumn{3}{|c|}{$\ldots$ low $\mathrm{S} / \mathrm{N}$} \\
\hline g & +15 & $170 \%$ & $4 .{ }^{\prime \prime} 6,3.2$ \\
\hline $\mathbf{k}$ & -50 & $31 \%$ & $2 ! .2,1.7$ \\
\hline I & -24 & $24 \%$ & $2 !{ }^{\prime \prime} 6,2.0$ \\
\hline m & -43 & $19 \%$ & 3."4, 2.6 \\
\hline
\end{tabular}

${ }^{a}$ Knots with significant amplitude of velocity gradient are marked in boldface.

${ }^{b}$ Gradients with positive sign have the highest velocity towards the north of the system.

${ }^{c}$ The seeing of $1^{\prime \prime}$ at the distance of AM 1353-272 A corresponds to $\sim 800 \mathrm{pc}$.

${ }^{d}$ We designate the region $\pm 1^{\prime \prime}$ around +45 "' 8 distance from the nucleus as a-to-b.

Note that nearly all of them are sites of active star formation, as predicted by a comparison of photometric starburst models with optical broad band colors.

At first glance, it seems as if there are velocity gradients within the knots and sudden jumps in velocity between them. To further analyze the step-like shape of the velocity curve, we subtract the overall streaming motion of the tails, by fitting a 5th order cubic spline (using IRAF's curfit task) to all velocity points. We then extract individual regions from the residual velocity field (see lower part of Fig. 60). In order to quantify the remaining apparent velocity gradients, we tried to fit a linear relation to all velocity gradients within the spatial extent of the knots. The results are given in Table 25, together with the error given by the fit procedure, the maximum velocity difference $\Delta V_{\max }$, and the angular extent of the fitted gradient. The fits seem to be a good first order approximation to the real velocity gradients in most cases, and are shown in the bottom part of Fig. 60. Seven knots show velocity amplitudes that are significant, with errors below $50 \%$. They reach $340 \mathrm{~km} \mathrm{~s}^{-1}$ for object ' $\mathrm{a}$ '. For several knots the gradient is also clearly resolved, i.e. it is observed to extend more than twice the seeing of $1^{\prime \prime}$.

\section{Discussion and Conclusions}

Given the observed significant velocity gradients within seven knots of the tails of AM 1353-272 A, we discuss several possibilities of the origin of this specific velocity distribution.

Instrumental errors can be excluded as a cause of these gradients. The velocity profiles observed over very small angular sizes (a few arcseconds) cannot be explained with flexures or distortions of the instrument. The wavelength calibration has been 
carried out and checked with the procedure described in Sect. 2. As a result, any relative instrumental effects within a given slit should be below $1 \mathrm{~km} \mathrm{~s}^{-1}$.

The apparent gradients could result from projection effects. As the angular size probed is only about 2 to 4 times the seeing, several smaller, physically unrelated knots might appear blended into one larger clump, mimicking the gradient we observe. It seems improbable, however, that this should be the case for many knots. Besides the two well determined tidal tails, there is no indication of complex tidal structures in this system. It is therefore quite unlikely that tidal debris outside the well-defined tails is observed in projection close to so many tidal knots.

Projection effects within a single tail are possible. First, the tails may have some depth along the line of sight, as can be seen in dynamical models of interactions starting with the idea of Toomre \& Toomre (1972) to view tails as two-dimensional ribbons. Second, the very tip of the southern tail could indeed be bent. Its threedimensional shape might partly account for the exceptionally large velocity gradient observed towards condensation ' $a$ '. An example of a projection by such a bend near the end of a tail is discussed by Hibbard et al. (2001) for the tail of the Antennae galaxies (NGC 4038/39). There, the total H I column density and the velocity dispersion along the line of sight mimic a massive condensation of matter. In their high-resolution $\mathrm{H}$ I data, they find two gas concentrations at different velocities, which have only about a tenth of the originally estimated mass. The amplitude $\Delta V_{\max }=340 \mathrm{~km} \mathrm{~s}^{-1}$ within knot 'a' (compared to $\Delta V_{\max } \sim 50 \ldots 100 \mathrm{~km} \mathrm{~s}^{-1}$ in the Antennae region) makes it seem unlikely to be caused by projection alone. The overall velocity field towards the end of the southern tail is decreasing. If the tail was bent backwards to the center, one would expect this trend to continue. Knots projected from this bent part of the tail should then have even lower velocity than 'a'. Such velocities are not observed. Projection effects by a bent tail for other knots apart from 'a' are implausible.

Velocity gradients could, in principle, be caused by gaseous outflows where one side of an expanding shell is blocked by dust. This would require significant amounts of extinction within the knots, while we find only very moderate absorption $\left(A_{B} \lesssim 1.0 \mathrm{mag}\right.$ including galactic $A_{B}=0.26 \mathrm{mag}$, estimated from Balmer line ratios). Additionally, an outflow would also imply line-broadening. There is no indication of broad lines here, as the FWHM of the H $\beta$ line is the same as the spectral resolution. It is very unlikely that the gradients are caused by outflows.

Rotation of bound objects could also create this kind of velocity gradients. From the irregular optical appearance and the location of the knots within tidal tails, it is unlikely that the stars in the potential of a knot have stable orbits. True Keplerian motion will not apply here, and it would then be premature to interpret the observed 
kinematics in terms of "rotation". Mass estimates from the Virial theorem would strongly overestimate the real mass contained within the knots ${ }^{4}$.

Instead, we may be witnessing the formation of bound objects in the tidal tails which are not yet virialized. The velocity gradients also seem to be specifically oriented in the sense that the higher velocity end of each knot points towards the center of AM 1353-272 A. A possible cause could be the rotational direction of progenitor disk before the interaction. The presence of the companion galaxy ' $\mathrm{B}$ ' near the southwestern tidal tail could also amplify motions in the tails, to create a "spin" in these knots in one direction. This would also explain the higher velocity amplitudes within the knots in the southwestern tail close to the companion.

The idea of Tidal Dwarf Galaxies (TDGs) — dwarf galaxies that are born as condensations in tidal tails - has been discussed in recent years by several authors (Barnes \& Hernquist 1992, Hibbard \& Mihos 1995, Duc et al. 1997), both theoretically and observationally. Duc et al. (2000) proposed a first definition of the term TDG as a self-gravitating object of dwarf galaxy mass formed from tidal material (see also Weilbacher \& Duc 2001). While we are hampered by the spatial resolution and cannot give the ultimate proof of the velocity gradients as motion of tidal material beginning to get bound into knots, it seems that we are witnessing the formation of TDGs in the tails of AM 1353-272 A, for the first time for multiple objects along both tails of the same interacting galaxy.

Forthcoming instruments for high-resolution spectroscopy on 8m-class telescopes, e.g. integral field instruments, will enable us to confirm the velocity gradients and further investigate their origin. Coupled with the high spatial resolution of adaptive optics these will provide very useful tools to analyze the unique properties of the knots seen in these tidal tails. To fully understand the geometry and interaction parameters causing this first series of possibly genuine Tidal Dwarf Galaxies and to complement the observations, detailed dynamical N-body+SPH modeling of the collision will be necessary.

We thank I. Appenzeller for help with the proposal and data retrieval and our anonymous referee for a friendly and helpful report. PMW is partly supported by DFG grant FR 916/62.

\section{References}

Barnes, J.E., Hernquist, L., 1992, Nature 360, 715

\footnotetext{
${ }^{4}$ If caused by Keplerian rotation alone, the rotational velocity of knot 'a' would be of the same order as those of giant spiral galaxies, which is unrealistic for an object with $M_{B}=-13.7$ mag.
} 
Duc, P.-A., Brinks, E., Springel, V., et al., 2000, AJ 120, 1238

Duc, P.-A., Brinks, E., Wink, J.E., Mirabel, I.F., 1997, A\&A 326, 537

Hibbard, J.E., Mihos, J.C., 1995, AJ 110, 140

Hibbard, J.E., van der Hulst, J.M., Barnes, J.E., Rich, R.M., 2001, AJ 122, 2969

Springel, V., White, S.D.M., 1999, MNRAS 307, 162

Toomre, A., Toomre, J., 1972, ApJ 178, 623

Weilbacher, P.M., Duc, P.-A., 2001, in: Dwarf Galaxies and their Environment (eds. K.S. de Boer, R.-J. Dettmar, U. Klein), 269-272

Weilbacher, P.M., Duc, P.-A., Fritze-von Alvensleben, U., 2002, A\&A In press

Weilbacher, P.M., Duc, P.-A., Fritze-von Alvensleben, U., Martin, P., Fricke, K.J., 2000, A\&A 358, 819 


\section{Part VI}

\section{Summary}

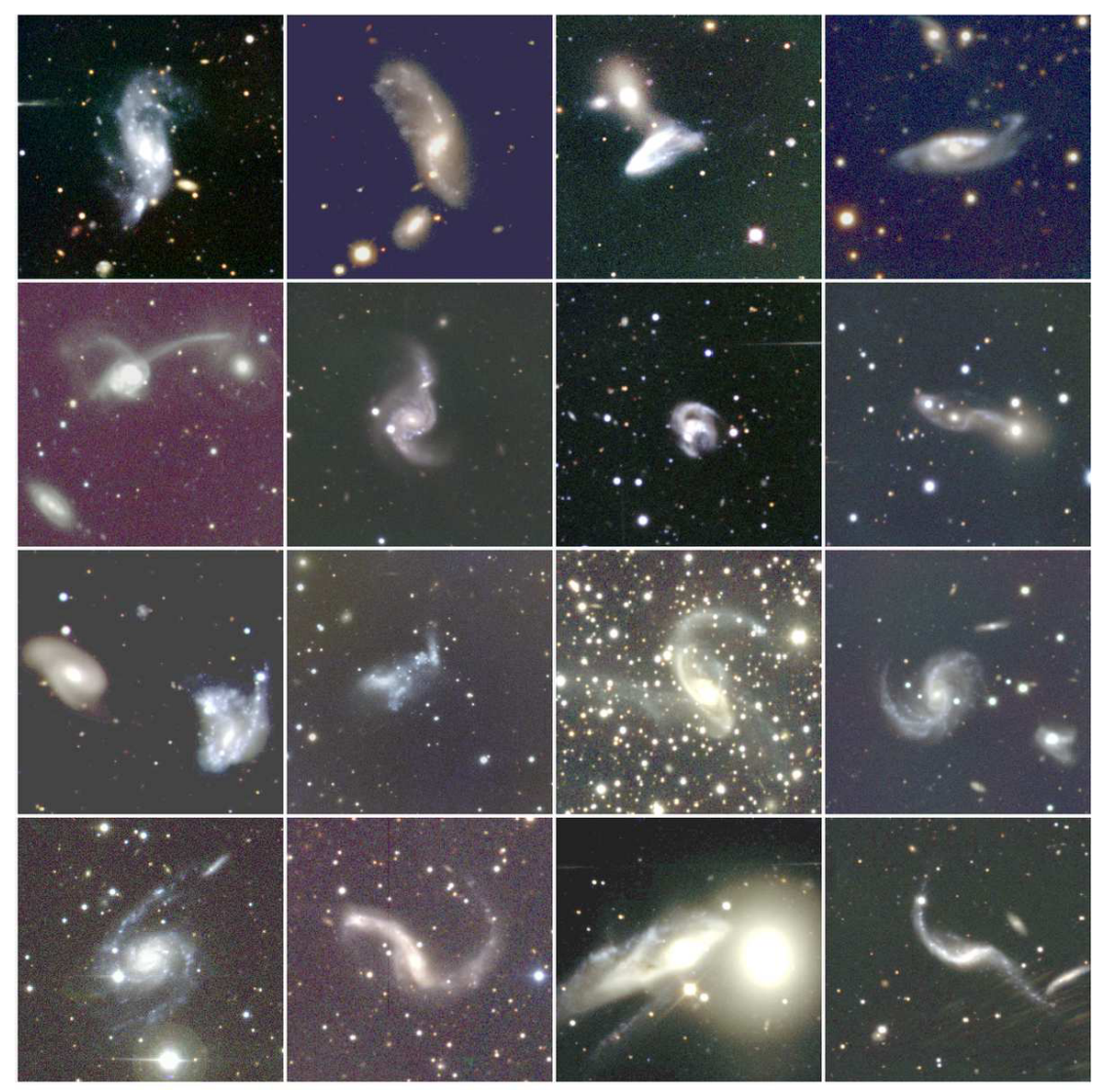





\section{Summary of the results}

This thesis deals with the so called Tidal Dwarf Galaxies (TDGs), a special class of dwarf galaxies which may form in the tidal features of interacting giant galaxies. Previous studies found several TDG candidates in various prototypical mergers with long tidal tails, and confirmed that some of those candidates show signs of internal kinematics and could be regarded as true TDGs, i.e. true galaxies in formation.

We present the first sample of TDG candidates (Paper I) in interacting galaxies on the southern hemisphere selected from the catalog Arp \& Madore (1987). Most of these galaxies have a chaotic morphology, and it is not easy to tell at a first glance which of the knots seen in the tidal features might be good TDG candidates. For the selection of good TDG candidates from optical imaging data, two-color photometry in combination with photometric evolutionary synthesis models was used. The color information and the comparison with the models enabled us to preselect promising TDG candidates against background objects. From the total of more than 100 individual knots in the tidal features, 44 objects were selected as candidates for TDGs based on optical imaging (Papers I and II). The photometric models also showed that TDGs can experience very strong starbursts and are expected to fade in luminosity by up to $2.5 \mathrm{mag}$ within the first $200 \mathrm{Myrs}$ after the burst.

Subsequent spectroscopic observations were conducted to confirm the association of the TDG candidates with the main interacting galaxies (Paper II). All candidates for which a redshift could be derived actually turn out to belong to the merging system. From the spectroscopy we could also derive Balmer line luminosities and equivalent widths, as well as oxygen abundances. The TDG candidates show H $\alpha$ luminosities between those of the most luminous $\mathrm{H}$ II regions in the disks of spiral galaxies and those of star-forming galaxies with $M_{B}>-19$ mag with a wide range of overlap. The oxygen abundance measurements confirm that TDGs do not follow the luminosity-metallicity relation of normal galaxies, but instead have a nearly constant metallicity of $12+\log (\mathrm{O} / \mathrm{H})=8.34$ with a $1 \sigma$ scatter of 0.20 dex. Three objects were excluded from our sample as TDG candidates because their low metallicity suggests that they are pre-existing objects nearby the interacting system which are either projected onto the tidal features or are falling into the interacting galaxies. However, the biggest surprise from the spectroscopy was that even with low spectral resolution we were able to detect velocity gradients in 13 TDG candidates. While the gradients could be caused by other effects - in particular projection - and have to be confirmed with higher resolution, they represent a first sign of decoupled kinematics required to call these knots genuine Tidal Dwarfs. 
Some of these velocity gradients were found with the low spectral resolution spectroscopy in the interacting system "The Dentist's Chair" (AM 1353-272). VLT observations with higher spectral resolution (Paper V) confirmed the existence of these gradients and revealed more knots with internal kinematics. In total, velocity gradients have been found in 7 knots, with velocity amplitudes up to $340 \mathrm{~km} \mathrm{~s}^{-1}$. The amplitudes of these gradients are too high to be interpreted by rotation alone, but part of the velocity could be caused by several smaller objects falling toward each other. We would then witness TDGs in the process of formation.

In Paper IV it is shown that the most frequently used indicator, the $\mathrm{H} \alpha$ luminosity $L(\mathrm{H} \alpha)$, for the current star formation rate (SFR), one of the basic parameters of composite stellar systems, is not applicable to systems with SFRs changing on short timescales $\left(\tau \leq 10^{7} \mathrm{yr}\right.$ ). For these systems, the offset of a few Myrs between the birth of a massive star and the highest output rate of ionizing Lyman continuum photons near shortly before its death is non-negligible, and the linear relation between $L(\mathrm{H} \alpha)$ and the SFR breaks down. Metallicities lower that the solar value important in particular for dwarf galaxies - and different stellar initial mass functions (IMF) add to this effect. In total, errors of up to two orders of magnitude in the determination of a rapidly changing SFR solely based on Balmer line luminosities are expected. Instead, we propose to use a spectrum with sufficient wavelength coverage or several broad-band optical/NIR filters in addition to the $\mathrm{H} \alpha$ luminosity and a comparison with spectrophotometric evolutionary synthesis models to derive a better estimate of the true star formation rate in dwarf galaxies.

The final combination of observations and modeling of the sample of TDGs is presented as Paper III. Here, all available data on the sample objects collected in the previous observing campaigns is further supplemented with deep near-infrared photometry in up to three filters. The evolutionary synthesis models have been extended to include the spectral evolution using stellar model atmosphere spectra of appropriate metallicity and gaseous emission (continuum and lines with new ratios valid for the different metallicities). We computed new spectrophotometric models for TDGs with different contributions of "old" stars inherited from the parent galaxy and young stars from the current starburst. The model of the appropriate metallicity was selected and compared with the measured $\mathrm{H} \beta$ equivalent width and the spectral energy distribution (SED) from up to 6 observed broad-band filters. The comparison with the models also enabled us to give a better estimate of the current star formation rate within the small TDG candidates with their short dynamical timescales, and allowed us to derive an estimate of the stellar mass of the knots. Finally, it was shown that in reality there is no strong dichotomy in the formation of these knots. For most objects it seems unlikely that they were build from purely gaseous or purely stellar condensations. Instead, a continuous range of stellar popu- 
lations, where between $\sim 10 \%$ and close to $100 \%$ of the mass of the TDG candidates are composed of young stars, is observed. The results indicate that one TDG candidate consists of only young stars while seven objects are at the beginning of a starburst and their contribution of young stars seems to be below 1\%. This also suggests, that the dominant formation process in our TDG candidates is similar to the model of Barnes \& Hernquist (1992), where TDGs are formed from stellar clumps as opposed to gaseous condensations (Elmegreen et al. 1993).

\section{Conclusions from this sample of TDGs}

A very basic result is the number of TDGs found in this investigation. From the original 44 good candidates from photometry, distributed over 14 interacting systems, redshifts were measured for 29 candidates and 26 passed the metallicity criterion, half of which also show signs of internal kinematics. If one believes that our sample is representative for galaxy interactions in the nearby universe, a first approximation that on average one true TDG is produced in every interaction can be estimated. This number, however, is not a final value, as follow-up observations have to confirm the velocity gradients. Other knots might also show signatures of internal kinematics if observed with the spectroscopic slit at a different position angle or with higher resolution.

As opposed to earlier investigations we distinguish between different "confidenceclasses" of objects in tidal features. The knots or condensations found in images of interacting galaxies in principle could be: background objects in projection, (globular) star clusters, (giant) $\mathrm{H}$ II regions, and TDG candidates. We call a knot a TDG candidate when it was shown that the colors from at least 3 filters match those of evolutionary synthesis models for nearby starbursts and the object is positioned in the tidal features. The last stage is that of genuine or true Tidal Dwarfs. To prove this, velocity measurements have to be carried out which show the TDG candidate to be self-gravitating, i.e. having internal kinematics decoupled from the surrounding material which is not caused by other effects. This is the most difficult part and has not yet been proven unambiguously for any TDG candidates.

The procedure that was developed in the course of this work to select TDG candidates against other knots is based on the optical data collected in the first two papers and detailed in Sect. 4 of Paper II. It involves the combination of multi-color imaging combined with evolutionary synthesis modeling and low resolution spectra. It proved to be very useful to confirm association, single out pre-existing dwarfs by their low metallicity, and select the best candidates for follow-up observations with higher spectral and spatial resolution. The $\mathrm{H} \alpha$ luminosity is not a good criterion for 
discriminating between TDGs and "normal" giant H II regions, but the equivalent width can be effectively used to age-date the star formation episode in the TDGs in combination with spectrophotometric evolutionary models. The high-resolution observations will then be able to confirm whether or not the velocity gradients discovered with low resolution are signs of truly decoupled motion, and hence, the condensation in question is a genuine Tidal Dwarf Galaxy.

As a by-product of the evolutionary synthesis models created for TDGs it was found that the H $\alpha$ luminosity is not a good tracer of the current star formation rate - in contrast to what is widely assumed - for any kind of system that experiences significant changes in its star formation rate on timescales shorter than $10^{7} \mathrm{yr}$ (e.g. dwarf galaxies, star formation regions, subgalactic fragments).

\section{Future Research}

While the current sample already allows to draw first conclusions on the typical number of TDGs produced in a galaxy interaction and on the properties of the stellar populations in the knots, the differences between individual knots, even within one interacting system, are significant. The final sample of objects for which decoupled kinematics have been found consists of only 13 objects. This number will even shrink, because the velocity gradients in some of these knots might be caused by the projection effects that we extensively discussed in Papers II and V. A larger sample would certainly help to put these results on a firmer basis, before one can reliably extrapolate the results for local TDGs to those that form at higher redshift.

The mode of star formation within TDGs is mostly unknown. Do they form stars mainly in dense star clusters as observed for the starburst in the centers of many interacting galaxies (Whitmore et al. 1999) and also in dwarf starbursts (O'Connell et al. 1994)? Or do they form stars at a lower level, spread out over the whole knot? While the most nearby TDG is well resolved (Duc et al. 2000), it appears to not have formed many young stars. High resolution multi-color imaging with the HST will be able to show in more active TDGs at larger distances in which ratio these two star formation modes occur.

As already pointed out, the internal kinematics are discovered with low spectral resolution and need confirmation with resolutions which yield accuracy better than the amplitude of the gradient. Fabry-Perot observations targeting the $\mathrm{H} \alpha$ line (e.g. Mendes de Oliveira et al. 2001) not only allow for very high spectral resolution but also give a two-dimensional distribution of the velocity and are therefore well suited for this task. All current indications of these kinematics are only seen in the 
bright lines emitted by the ionized gas associated with the TDGs. The true dynamical status of the knots can only be judged from the measurement of kinematics in the stellar component, i.e. from absorption lines. Very deep spectroscopy with large telescopes will be necessary to reach good enough $\mathrm{S} / \mathrm{N}$ in the continuum. If absorption lines are detected with adequate spectral resolution, the kinematics in the stellar component can be studied and velocity dispersion could be measured in addition to velocity gradients.

One of the missing pieces in the theoretical understanding of the formation of TDGs is the lack of modern high-resolution dynamical simulations. The reference simulations by Barnes \& Hernquist (1992) and Elmegreen et al. (1993) are by now 10 years old and in this time the number of particles routinely used in dynamical models of interacting galaxies has increased significantly (up to a factor of 10 or more). The models also begin to include recipes for star formation and feedback (Springel 2000). A few models matching specific interacting systems have been computed (Hibbard \& Mihos 1995, Duc et al. 2000), and some authors even note the existence of knots in the tidal tails while investigating other properties of mergers (e.g. Naab \& Burkert 2001). But neither parameter studies — to investigate the circumstances under which TDGs are most likely to form or not - nor high-resolution models, which in detail study the formation and survival of condensations in the tidal tails, have been carried out.

Besides the possibility of studying young galaxies in the local universe, one would also like to assess the cosmological significance of TDGs with these investigations. For the extrapolation to higher redshifts, extensive additional modeling seems to be required, to complement observations of both more detailed nature and a larger sample of objects. Chemical evolution models for the parent galaxies (e.g. Lindner et al. 1999) will tell how far back in redshift TDGs formed in interactions or during merger events will still stand out in metallicity above the normal dwarf galaxy population at that redshift. Ultimately, this will allow conclusions about the contribution of Tidal Dwarf Galaxies to today's dwarf galaxy population.

\section{References}

Arp, H.C., Madore, B.F., 1987, A Catalogue of Southern Peculiar Galaxies and Associations, Cambridge University Press, Cambridge

Barnes, J.E., Hernquist, L., 1992, Nature 360, 715

Duc, P.-A., Brinks, E., Springel, V., et al., 2000, AJ 120, 1238 
Elmegreen, B.G., Kaufman, M., Thomasson, M., 1993, ApJ 412, 90

Hibbard, J.E., Mihos, J.C., 1995, AJ 110, 140

Lindner, U., Fritze-von Alvensleben, U., Fricke, K.J., 1999, A\&A 341, 709

Mendes de Oliveira, C., Plana, H., Amram, P., Balkowski, C., Bolte, M., 2001, AJ 121, 2524

Naab, Thorsten, Burkert, Andreas, 2001, in: Galaxy Disks and Disk Galaxies (eds.

J.G. Funes, E.M. Corsini), ASP Conf. Ser.

O’Connell, R.W., Gallagher, J.S., Hunter, D.A., 1994, ApJ 433, 65

Springel, Volker, 2000, MNRAS 312, 859

Whitmore, B.C., Zhang, Q., Leitherer, C., et al., 1999, AJ 118, 1551 




\section{Frequently used acronymns and abbreviations}

\begin{tabular}{ll} 
BCD & Blue Compact Dwarf galaxy \\
CMD & Color-Magnitude Diagram \\
DM & Dark Matter \\
dSph & dwarf Spheroidal galaxy \\
ESO & European Southern Observatory \\
EW & Equivalent Width \\
Gyr & Billion years (10 ${ }^{9}$ yr) \\
HST & Hubble Space Telecope \\
IMF & Initial Mass Function \\
Myr & Million years $\left(10^{6}\right.$ yr) \\
NIR & Near-InfraRed \\
NTT & New Technology Telecope \\
SED & Spectral Energy Distribution \\
SF & Star Formation \\
SFR & Star Formation Rate \\
TDG & Tidal Dwarf Galaxy \\
UV & Ultra-Violet \\
VLT & Very Large Telecope \\
\hline & \\
$H_{0}$ & Hubble constant \\
$\Omega_{M}$ & Matter density of the universe \\
$\Lambda$ & Cosmological constant \\
$\Omega_{\Lambda}$ & Density attributed to the cosmological constant \\
$b$ & burst strength (young stellar mass to total stellar mass) \\
$B$ & burst strength (young stellar mass to “old” stellar mass) \\
$L(H \alpha)$ & H $\alpha$ luminosity \\
EW(H $\beta)$ & redshift \\
$z$ & Melocity \\
$V$ & oxygen abundance in logarithmic units \\
$Z$ & \\
$12+\log (\mathrm{O} / \mathrm{H})$ &
\end{tabular}





\section{Danksagung}

Wenn ich auch die eigentliche Arbeit für diese Dissertation komplett selbst gemacht habe, so haben doch viele Personen dazu beigetragen, daß ich diese Dissertation fertigstellen konnte.

Uta Fritze-v. Alvensleben hat sich trotz anderer Verpflichtungen immer Zeit für mich genommen und Verständnis für alle wissenschaftlichen und sonstigen Probleme gehabt. Ihr großer Überblick über die vorhandene Literatur verschiedener Themengebiete und ihre langjährige Erfahrung mit den Evolutionssynthesemodellen haben mir sehr geholfen. Außerdem war sie immer begeistert davon, zu welchen Ergebnissen die Arbeit an diesem Thema geführt hat, manchmal mehr als ich selbst.

Pierre-Alain Duc hat mir trotz der großen Entfernung in schwierigen Situationen immer wieder sehr kompetent und schnell geholfen, manchmal mehrmals täglich Emails mit mir ausgetauscht und in langen Telefonaten die wichtigsten Dinge besprochen. Er hat sich bei mehreren Besuchen in Cambridge und Saclay nett um mich gekümmert, mir viel über Datenreduktion und -auswertung beigebracht, und mich bei meiner ersten Beobachtungskampagne auf La Silla in Chile unterstützt.

Ich danke Prof. Dr. Klaus J. Fricke für die Ermöglichung der Arbeit in der Abteilung II der Sternwarte Göttingen und für die Übernahme des Korreferats dieser Arbeit. Er hat großzügig die letzten Stunden garantierter Beobachtungszeit am VLT für mein Projekt zur Verfügung gestellt.

Meinem Zimmergenossen Karsten Bischoff danke ich für die angenehme Atmosphäre, Tips zur Datenanalyse und grundsätzliche Diskussionen nicht nur zur Astronomie. In der letzten Zeit war Luz-Marina Cairós-Barreto eine freundschaftliche Zimmergenossin und hat gut dafür gesorgt, daß ich mein Englisch nicht nur in Schriftform geübt habe.

Polychronis Papaderos hat mich immer hilfsbereit unterstützt und war genauso wie Kai Noeske ein guter Gesprächspartner zu allen Aspekten von Zwerggalaxien. Mit seiner großen Erfahrung in der Analyse von Galaxienspektren hat mir auch Wolfram Kollatschny einige wertvolle Tips geben können. Außerdem wußte er immer interessante Neuigkeiten zu berichten. 
Neben Uta waren mir auch die anderen Mitglieder der Galaxienentwicklungsgruppe, Peter Anders, Jens Bicker, Thomas Lilly, Claudia Möller und Jochen Schulz auch außerhalb der legendären „Schokobrötchentreffen“ eine große Hilfe.

Ohne die anderen Computeradministratoren der Sternwarte, Ingo Berentzen, Jens Kube, Klaus Reinsch, Wilfried Steinhof, die teilweise freiwillig ihre knappe Freizeit zur Installation und Wartung der Computer zur Verfügung gestellt haben, wäre das Netzwerk der Sternwarte schon lange zusammengebrochen.

Allen anderen nicht namentlich erwähnten netten Kollegen aus der Sternwarte danke ich für die gute Arbeitsatmosphäre.

Meiner lieben Frau Anna habe ich leider nur einen Bruchteil meiner Arbeit verständlich machen können. Trotzdem hat sie während der gesamten Zeit meiner Doktorarbeit große Geduld bewiesen und mich vor allem in der letzten, kritischen Phase gut mit Essen versorgt. Schließlich war sie als Testhörerin für den Disputationsvortrag eine große Hilfe.

Auch wenn ich leider viel zu selten und zu kurz in Holzminden gewesen bin, waren die Besuche bei meinen Eltern und die Treffen mit meinen drei Geschwistern und allen anderen Mitgliedern der „Großfamilie“ immer sehr entspannend, haben mich oft wieder aufgemuntert und manchmal aus den himmlischen Sphären wieder auf den Boden der Tatsachen zurückgeholt. Meinen Eltern danke ich ganz besonders, daß sie mich in der Zeit meines Studiums und der Promotion nicht nur finanziell sondern auch besonders moralisch unterstützt haben.

Ich danke auch meinen Studienfreunden, vor allem Alexander Meyer, Ulrich Vogt und Marek Wieland ohne deren Zusammenarbeit bei schwierigen mathematischen und physikalischen Problemen im Grund- und Hauptstudium ich wahrscheinlich nie bei einer Doktorarbeit angekommen wäre.

Johann Sebastian Bach, Loreena McKennitt, Wolfgang-Amadeus Mozart, Arvo Pärt, Camille Saint-Saëns, Sting, U2 und noch vielen anderen bin ich für wundervolle Musik dankbar, die mir vor allem geholfen hat, kurz vor der Abgabe auch nachts um 4 Uhr noch wach zu bleiben. 


\section{Curriculum Vitae}

Name Peter Michael Weilbacher.

Birth 07 July 1973, Holzminden, Germany.

Parents Rita and Norbert Weilbacher.

Nationality German.

Marital status Married with Anna K. Weilbacher.

1980-Jul. 1984

1984-Jun. 1986

1986-May 1993

Oct. 1994-Jul. 1997

Sep. 1996

Oct. 1996-Jul. 1997

Aug. 1997-Dec. 1998

Feb. 1999

since May 1999

May-Oct. 1999

Jul.-Sep. 1999

since Mar. 1999
Kath. Grundschule, Holzminden.

Orientierungsstufe Liebigstraße, Holzminden.

Campe-Gynmasium, Holzminden.

Finished school with the "Abitur" (grade "gut")

Studies of Physics, University of Osnabrück.

"Vordiplom" in Physics (grade "gut")

Studies of Physics, University of Göttingen.

Diploma project, Universitäts-Sternwarte Göttingen.

Title: Photometry and Modelling of Interacting and

Tidal Dwarf Galaxies.

Advisor: Prof. Dr. Klaus J. Fricke.

Diploma degree in Physics (grade "sehr gut").

Research assistant, Universitäts-Sternwarte Göttingen.

Work in the Göttingen FORS group

Conference organization for the national astronomy meeting (“AG-Tagung") in Göttingen.

PhD project, Universitäts-Sternwarte Göttingen.

Title: On the Formation and Evolution of Dwarf Galaxies in Tidal Tails.

Advisor: Priv.-Doz. Dr. Uta Fritze-von Alvensleben. 